\title{
Finite Element Methods to Analyze Helical Stent Expansion
}

\author{
by \\ Nasim Paryab \\ A thesis \\ presented to the University of Waterloo \\ in fulfillment of the \\ thesis requirement for the degree of \\ Doctor of Philosophy \\ in \\ Mechanical Engineering
}

Waterloo, Ontario, Canada, 2014

CNasim Paryab 2014 


\section{AUTHOR'S DECLARATION}

I hereby declare that I am the sole author of this thesis. This is a true copy of the thesis, including any required final revisions, as accepted by my examiners.

I understand that my thesis may be made electronically available to the public. 


\begin{abstract}
Stent angioplasty or stenting is the standard noninvasive therapy method to address narrowing of the arteries and restore blood flow. Although drug-eluting stents have resolved immediate restenosis (i.e., re-narrowing of the stented arteries), late in-stent restenosis continues to provide challenges for this treatment. One way to address this is through the use of biodegradable stents, such as the helical stent configuration investigated in this work, which may reduce the risk of late in-stent restenosis.

The focus of this study was to analyze the mechanical performance and expansion of a polymeric helical stent, applied to a coronary artery. Experimental testing of such a device presents many challenges and is costly. Hence, initial evaluation using numerical methods provides an opportunity to investigate some aspects of the mechanical performance in detail. Existing stent expansion modeling methods originally developed for metallic wire mesh stent simulations were used to investigate the response of a 5 coil polymeric helical stent. The methods include: prescribed displacement, uniform expansion and balloon expansion. It was determined that only the balloon expansion could capture the important aspects of the helical stent expansion such as non-uniform expansion, foreshortening and change in pitch observed in experimental tests, since the expansion mechanism of this stent is different compared to traditional stents. More important, it was found that the helical stent expansion was limited by non-uniform expansion (dog boning) and a unique progressive expansion method was proposed to mitigate this issue. Finally, the stent and progressive balloon expansion models were used to investigate helical stent expansion within an artery containing plaque. The models predicted that interaction with the artery decreased foreshortening compared to free expansion of the stent. An artery with plaque demonstrated higher stresses in the vicinity of the plaque and modest stresses outside of this region, which are desirable in terms of reducing the occurrence of restenosis. The modeling techniques developed in this work have allowed for the evaluation of a novel polymeric biodegradable helical stent and progressive expansion method. The balloon expansion model was found to most accurately predict the geometric effects of expansion and, although this particular stent geometry demonstrated some challenges in terms of non-uniform expansion, the stresses in an artery with plaque were relatively low outside the plaque zone making this a promising approach to address restenosis.
\end{abstract}




\section{Acknowledgements}

I would like to take this opportunity to express my deep gratitude and appreciation to those people who have helped me to complete this journey, and to individuals to whom I am very much indebted and who, without their support, this achievement would not have been possible.

First of all, special thanks to my supervisors, Professor Duane Cronin and Professor Pearl Sullivan for their great guidance, mentorship and encouragement. In particular, I was extremely lucky to have Dr. Cronin as my supervisor, who cared so much about my work and guided me throughout this study with patient. Also, I wish to express my thanks for the thoughtful review of Dr. Marcello Panini as the external examiner. I would like to thank the reading committee members, Dr. Hamid Jahed, Dr. Maud Gorbet, and Dr. Naveen Chandrashekar for their valuable comments and suggestions. Thanks go to all of my colleagues at the University of Waterloo who have contributed to make it such an enjoyable place to work.

Many thanks, to my friends with whom I have shared so much fun and so many good times over the past few years in the Kitchener-Waterloo region. Although I cannot mention them all by name, I am grateful to all of them and wish them the best. Many thanks to all the members of my family, particularly my brother, Amir, and my sister, Neda, for the warmth and kindness they always offer, with special gratitude to my dear parents for their continuous support and encouragement; I indebted my life to them. I am thankful to my son, Arman, for his magical smiles that fill my heart with joy and peace. Finally, my appreciations and thanks are dedicated to my Nasser, for always showing me that my dreams are his dreams and proving to me that impossible is not possible. 


\section{Dedication}

To my family. 


\section{Table of Contents}

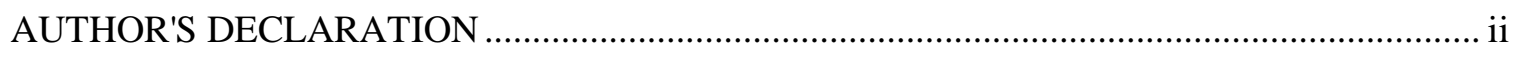

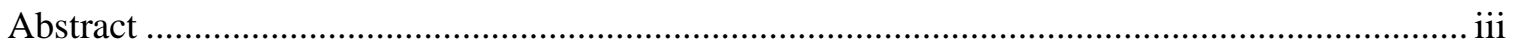

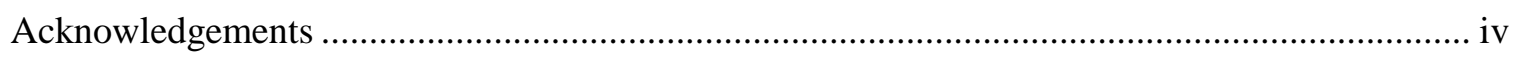

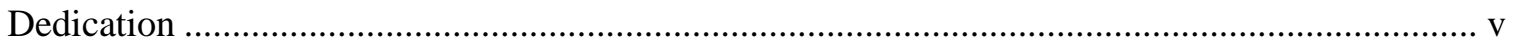

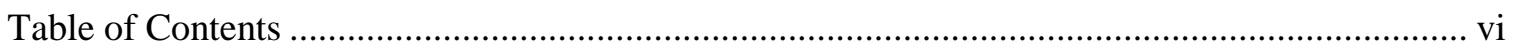

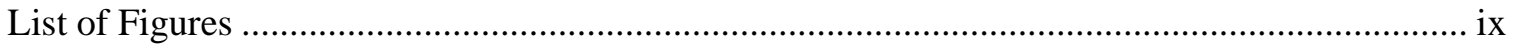

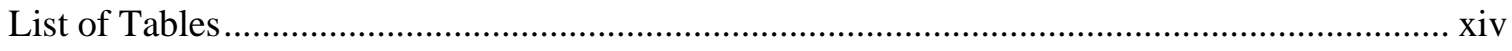

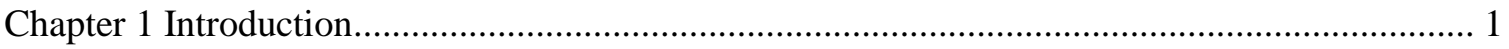

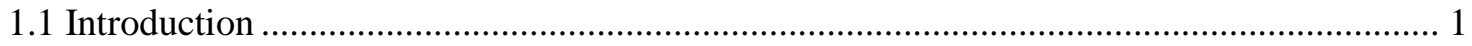

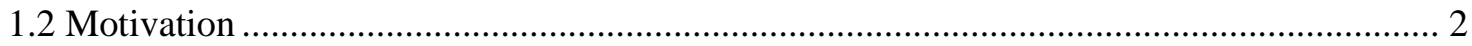

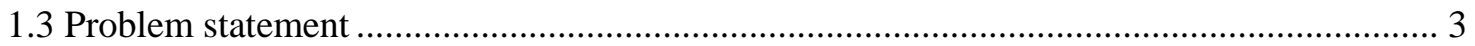

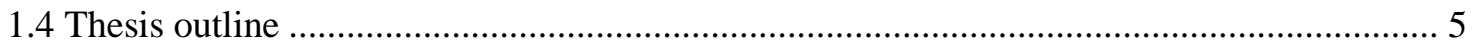

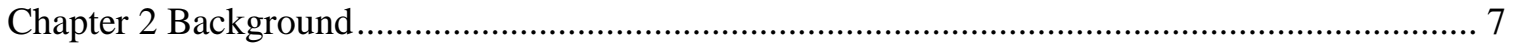

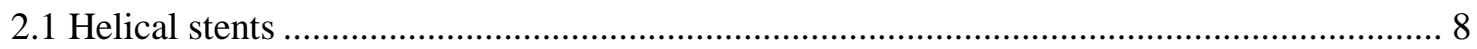

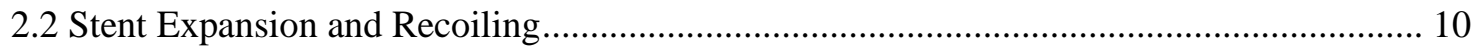

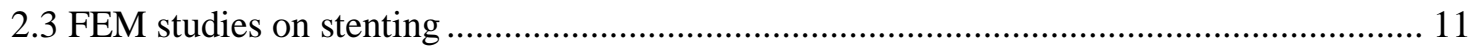

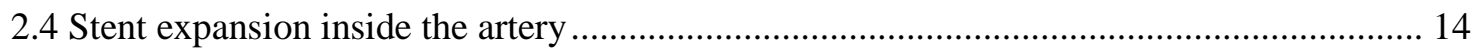

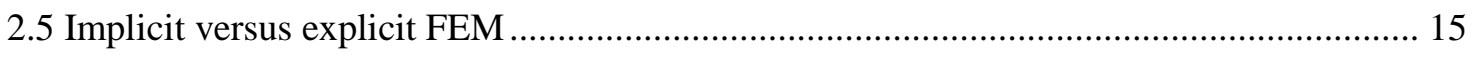

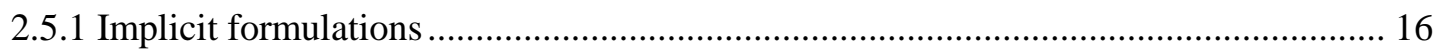

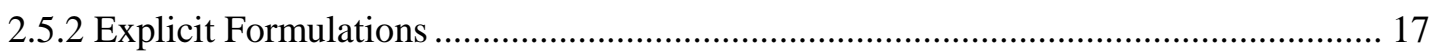

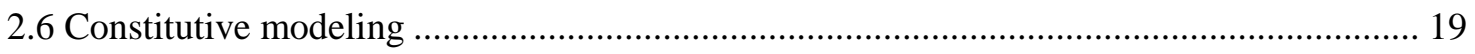

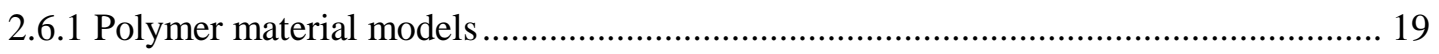

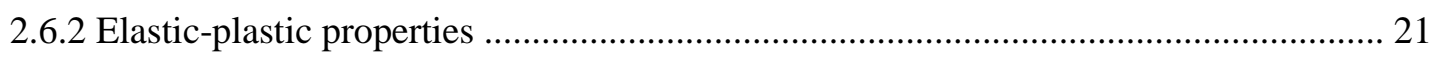

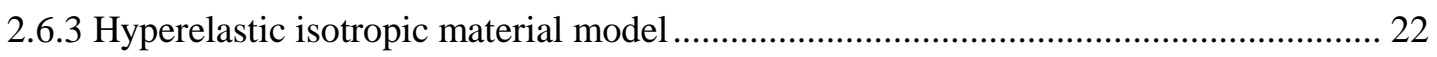

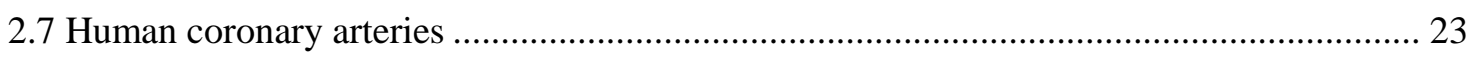

2.7.1 Acquiring mechanical properties of arteries......................................................... 27

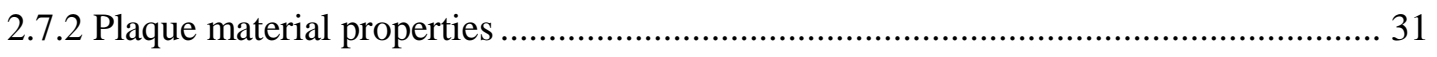

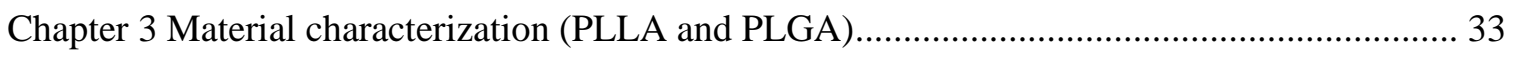

3.1 Dynamic mechanical analyzer (DMA) specifications .................................................... 33

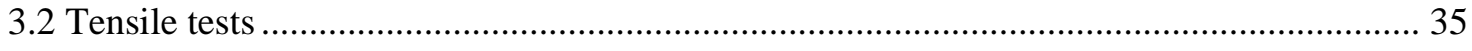

3.3 Presoaking effects on the stress-strain curve of PLLA samples ......................................... 40 


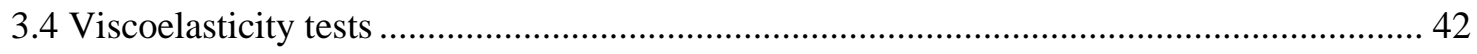

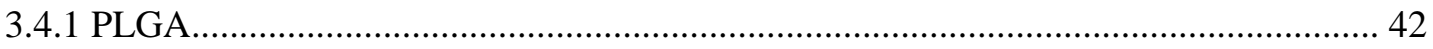

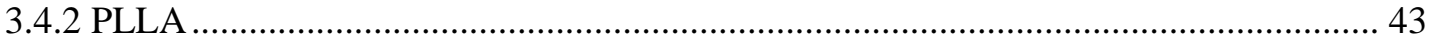

3.4.3 Evaluating linearity of PLLA samples in vitro (in $37^{\circ} \mathrm{C}$ water) ................................. 45

Chapter 4 Finite Element Approaches to Analyzing Helical Stent Expansion.............................. 49

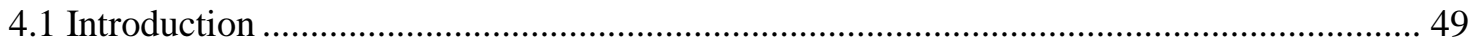

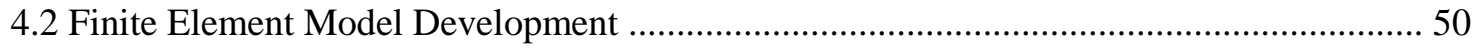

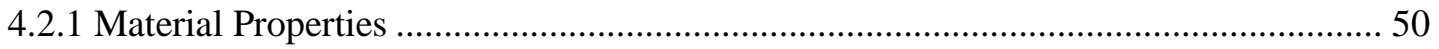

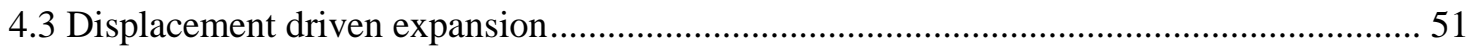

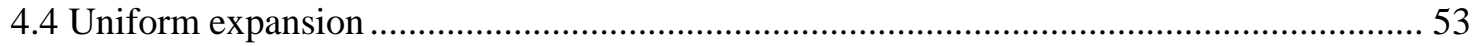

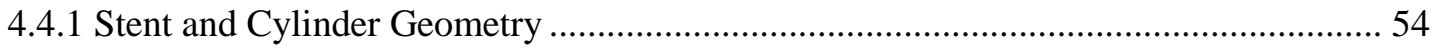

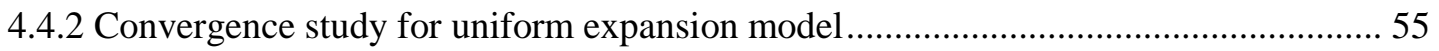

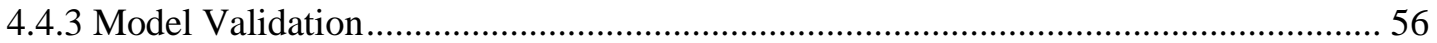

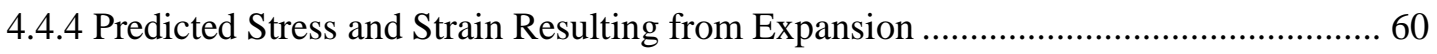

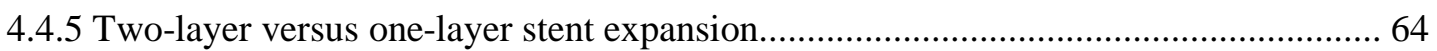

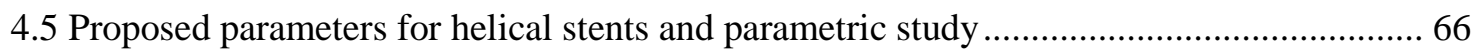

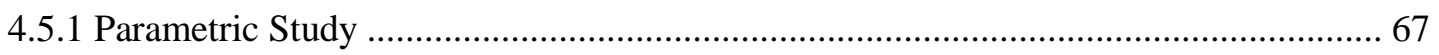

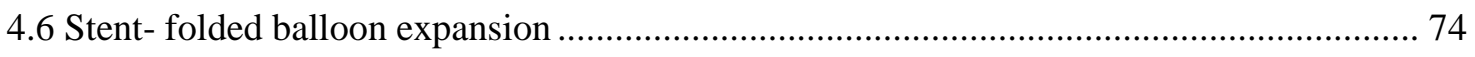

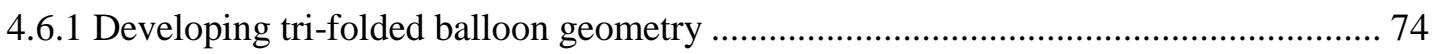

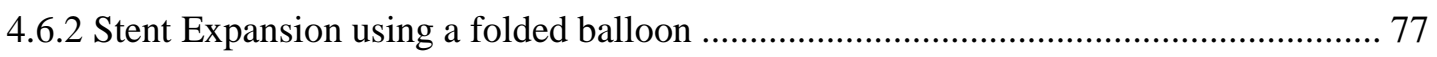

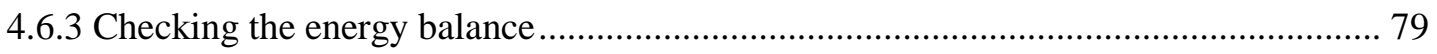

4.6.4 Typical balloon expansion of a helical stent .......................................................... 80

4.6.5 Unsymmetrical expansion of helical stent with folded balloon inflation ..................... 82

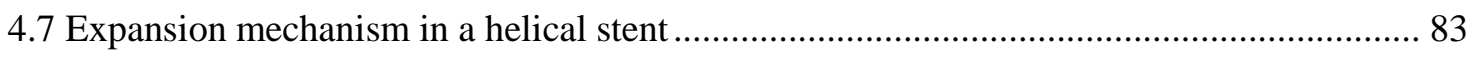

Chapter 5 Challenges to achieving full expansion of helical stent ............................................. 85

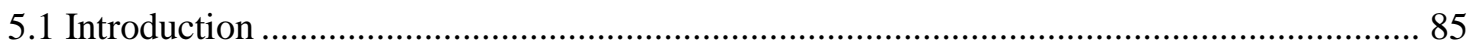

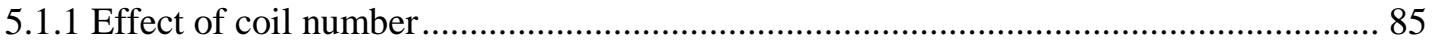

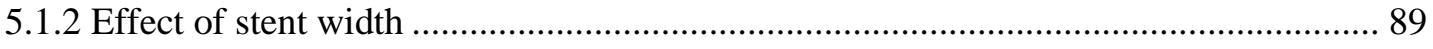

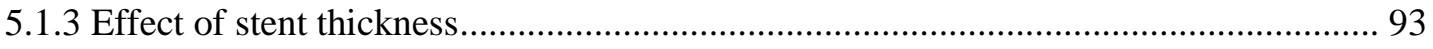

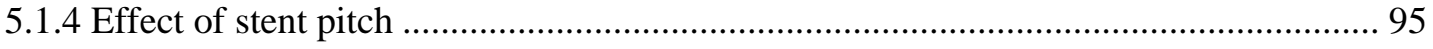

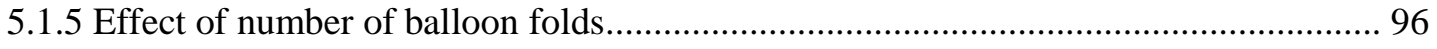

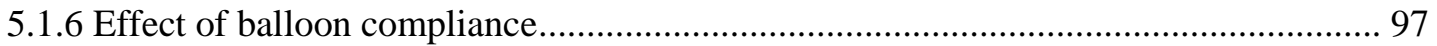

vii 


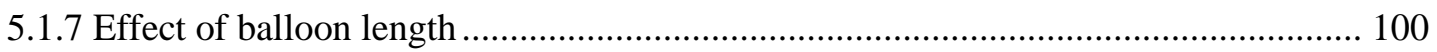

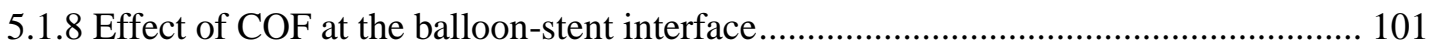

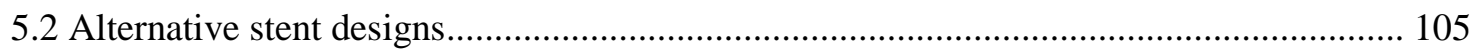

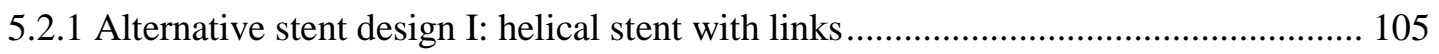

5.2.2 Alternative stent design II: helical stent with three sequentially connected two-coil

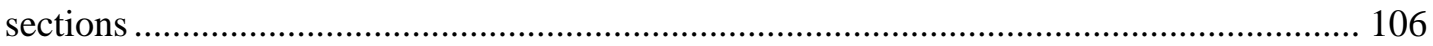

5.2.3 Alternative stent design III: three-coil sequential helical stent................................. 107

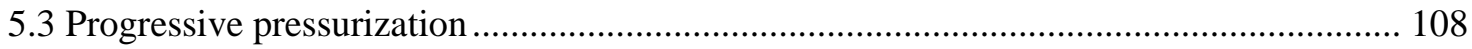

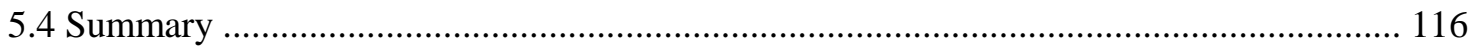

Chapter 6 Analysing helical stent deployment inside a human coronary artery with plaque buildup

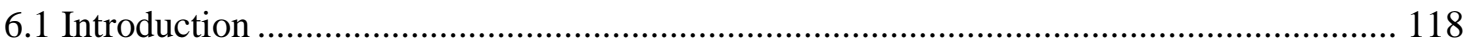

6.2 Development and verification of the artery and plaque constitutive model ..................... 119

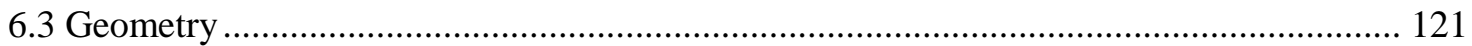

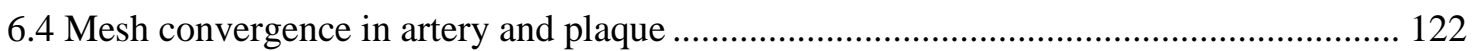

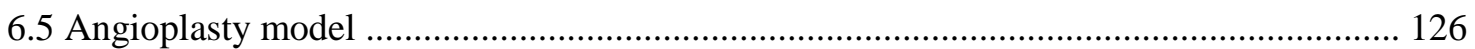

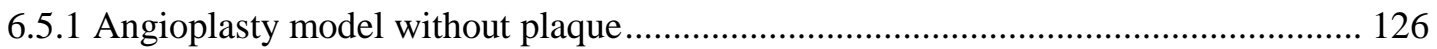

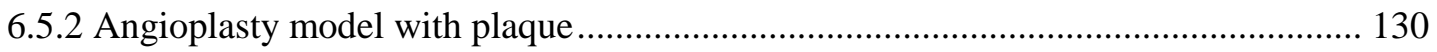

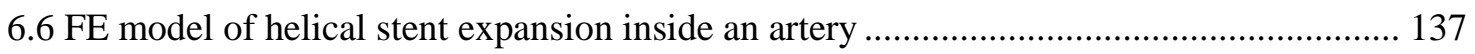

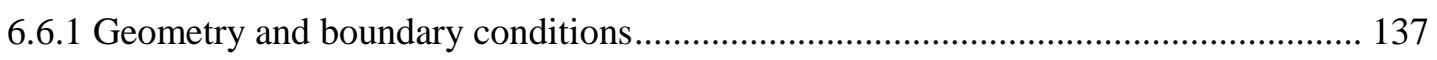

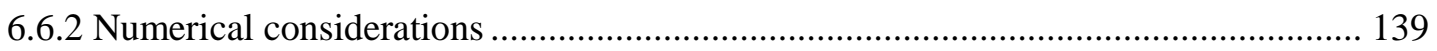

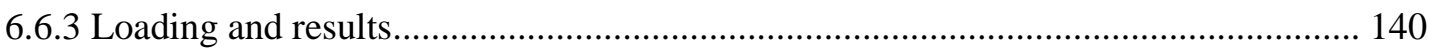

6.7 Potential injury areas in the artery and plaque post-expansion ..................................... 144

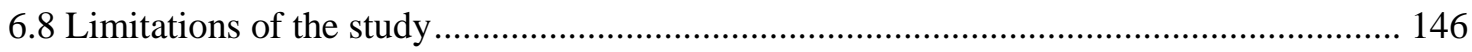

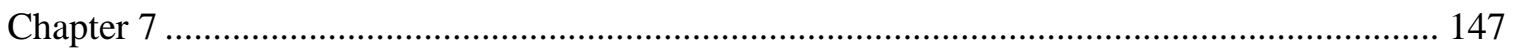

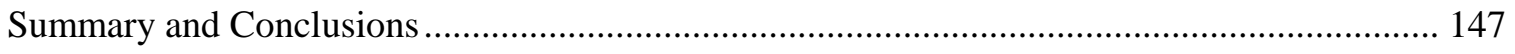

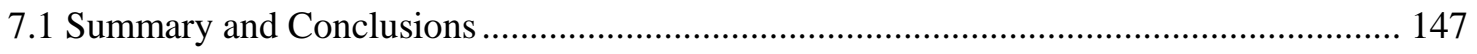

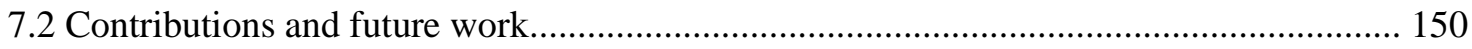

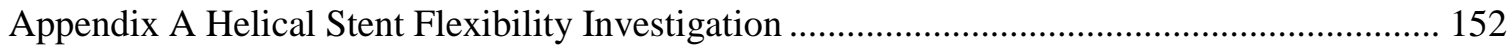

Appendix B Implicit versus explicit finite element modeling approach for analyzing stent uniform

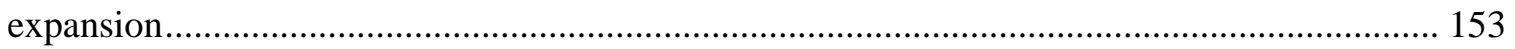

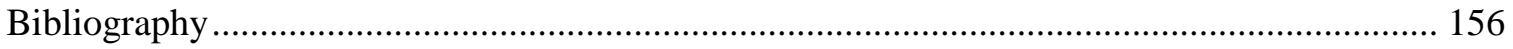




\section{List of Figures}

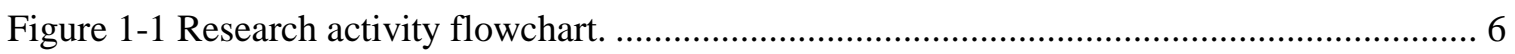

Figure 2-1 A typical helical stent [Venkatraman, 2003] ......................................................... 9

Figure 2-2 Balloon expansion of a helical stent in saline bath, demonstrating 'dog boning' and

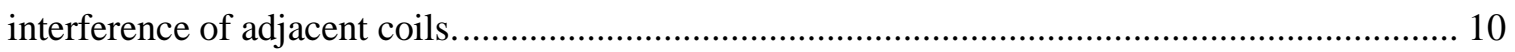

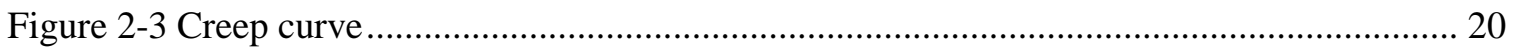

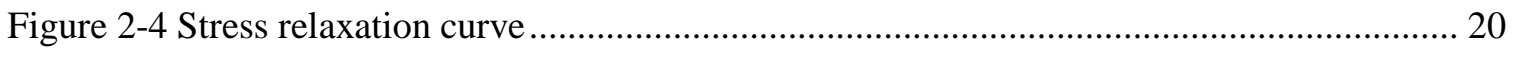

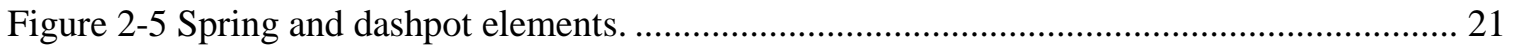

Figure 2-6 Schematic representation of the Ascending Aorta and its branching arteries

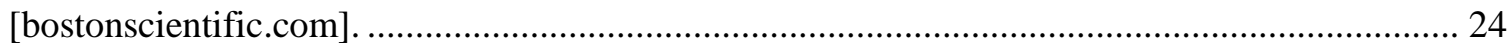

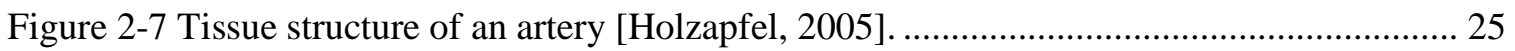

Figure 2-8 Typical true stress-stretch curve of an artery [from Claes, 2010].............................. 26

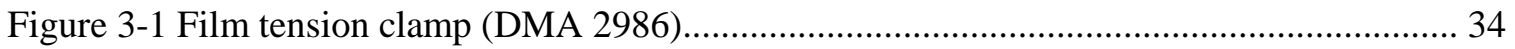

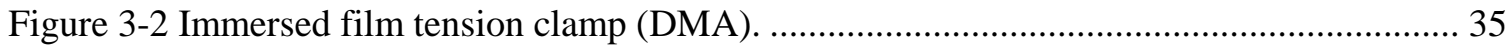

Figure 3-3 Stress-strain results for PLLA samples tested at $25^{\circ} \mathrm{C}$ and $37^{\circ} \mathrm{C}$ in air and water-bath using a DMA tension clamp. Error bars show the upper and lower limits observed during the experiment. The ' $\mathrm{x}$ ' in the figures indicates failure of the sample, while the arrow indicates that

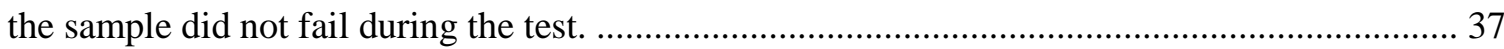

Figure 3-4 Stress-strain results of PLGA samples tested in $25^{\circ} \mathrm{C}$ and $37^{\circ} \mathrm{C}$ in air and in water-bath using a DMA tension clamp. Error bars show the upper and lower limits observed during the experiment. The ' $x$ ' in the figures indicates failure of the sample, while the arrow indicates that

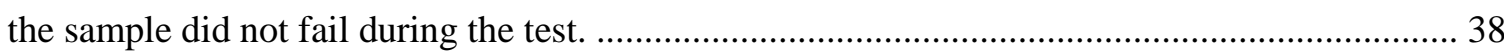
Figure 3-5 Image of a PLGA sample 24 hours after pre-conditioning and tension testing in a $37{ }^{\circ} \mathrm{C}$

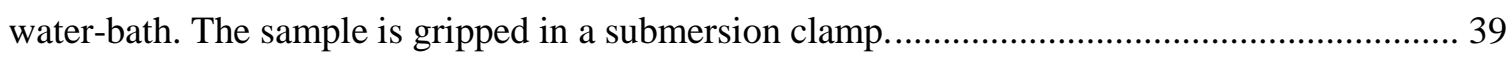

Figure 3-6 Tension tests to evaluate the effect of soaking time on the stress-strain curve of PLLA samples (one sample is tested for each condition).

Figure 3-7 Creep test on PLGA sample in $37{ }^{\circ} \mathrm{C}$ water under $2 \mathrm{MPa}$ load after $30 \mathrm{~min}$ soaking in water.

Figure 3-8 PLLA creep test results measured in room temperature air under $25 \mathrm{MPa}$ tensile

loading. 44

Figure 3-9 In vitro PLLA creep results (in the loading range of 10-35 MPa) following 30 minutes of preconditioning in $37^{\circ} \mathrm{C}$ water . 45 
Figure 3-10 Creep Compliance of PLLA samples in vitro.

Figure 3-11Creep compliance versus applied stress measured 10 min after applying the force... 47

Figure 3-12 Recovery compliance curves of PLLA samples tested in vitro.

Figure 3-13 Recovery compliance versus stress curves of PLLA samples measured after 10 min

of recovery. 48

Figure 4-1 Prescribed radial displacement on the inner surface of a five-coil stent, arrows denote

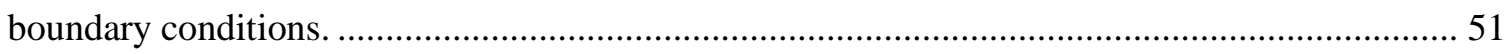

Figure 4-2 Predicted helical stent expansion using applied radial displacement. ........................ 52

Figure 4-3 Equivalent plastic strain for the displacement-driven expansion at model termination $(0.054 \mathrm{~mm}$ radial expansion). 53

Figure 4-4 Eight-coil helical stent geometry. 54

Figure 4-5 Convergence study based on maximum stress values, element size quoted as single element volume. 55

Figure 4-6 Convergence study based on maximum equivalent plastic strain and maximum principal strain, element size quoted as single element volume. 56

Figure 4-7 Unexpanded and expanded stent showing foreshortening and uncoiling. 57

Figure 4-8 Pressure versus diameter for expansion of an 8.5 coil stent. 58

Figure 4-9 Stent recoiling (outside diameter) following expansion and removal of balloon........ 59

Figure 4-10 Eight-coil stent model before expansion, after expansion and after recoiling........... 60

Figure 4-11 Circumferential stress distribution (in MPa) for an 8-coil stent after expansion....... 62

Figure 4-12 Circumferential stress distribution (in MPa) for an 8-coil stent after expansion along the thickness of the inner coil. 62

Figure 4-13 Circumferential stress distribution (MPa) for an 8-coil stent after recoiling. 63

Figure 4-14 Equivalent plastic strain distribution for an 8-coil stent after recoiling...... 63

Figure 4-15 Two-layer stent including PLLA (inner layer) and PLGA (outer layer) layers. 64

Figure 4-16 One-layer PLLA stent model. 65

Figure 4-17 von Mises stress changes during expansion of a one-layer PLLA stent and a two-

layer PLLA and PLGA stent. 66

Figure 4-18 Evaluation parameters as a function of number of coil. 68

Figure 4-19 Evaluation parameters as a function of COF. 69

Figure 4-20 Evaluation parameters as a function of stent width. 70

Figure 4-21 Evaluation parameters as a function of stent thickness. .71 
Figure 4-22 Evaluation parameters as a function of stent pitch. ............................................... 72

Figure 4-23 FE model of the balloon folding process (3-fold).................................................. 76

Figure 4-24 Verification of free expansion of a folded balloon.................................................. 77

Figure 4-25 FE model of a coupled stent and tri-folded balloon................................................. 78

Figure 4-26 Convergence study on the element size of the stent................................................ 79

Figure 4-27 A typical kinetic-to-total energy ratio model of helical stent expansion with folded

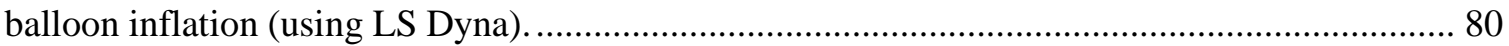

Figure 4-28 Typical helical stent expansion with a folded balloon (1.42 $\mathrm{mm}$ expansion)........... 81

Figure 4-29 Unsymmetrical expansion of a helical stent. The end shown here expands faster than the other because the preferred direction of the balloon is in the opposite direction of the stent

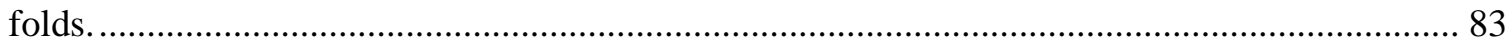

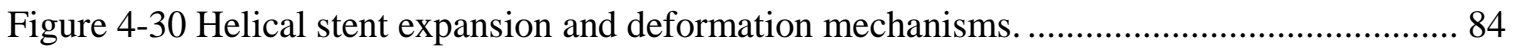

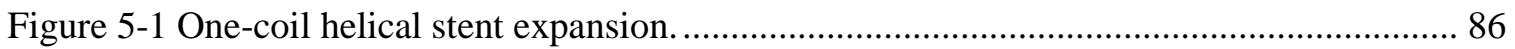

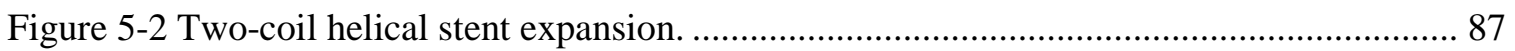

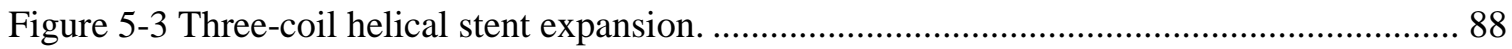

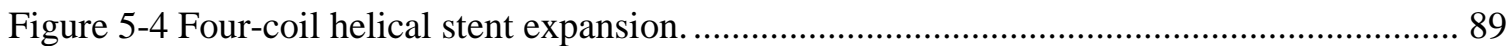

Figure 5-5 Expansion of a $0.12 \mathrm{~mm}$ (width) four-coil stent........................................................ 90

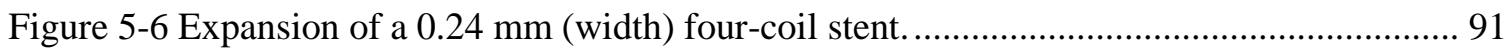

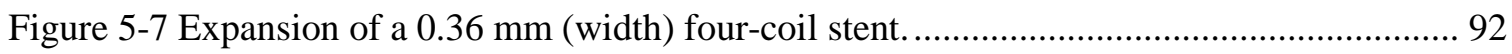

Figure 5-8 Expansion of a $0.03 \mathrm{~mm}$ (thick) four-coil stent. ...................................................... 93

Figure 5-9 Expansion of a five-coil stent with varied thickness. ................................................ 95

Figure 5-10 Expansion of a five-coil stent with pitch value of $1.2 \mathrm{~mm}$.................................... 96

Figure 5-11 Expansion of a six-coil stent using a balloon with four folds ................................... 97

Figure 5-12 Expansion of a four-coil stent with a non-compliance balloon. .............................. 98

Figure 5-13 Expansion of a five-coil stent using a tri-folded balloon with varied wall thickness.

Figure 5-14 Expansion of a four-coil stent with a short balloon. ............................................. 101

Figure 5-15 Expansion of a four-coil stent with $\mathrm{COF}=0.2$ at the stent-balloon interface. ........ 102

Figure 5-16 Expansion of a five-coil stent with a balloon bearing 50 times the modulus at its end

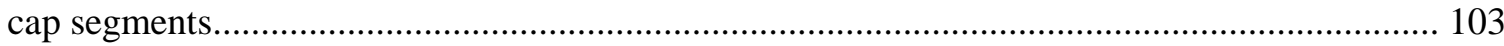

Figure 5-17 Expansion of a five-coil stent with a balloon bearing 100 times the modulus at its two

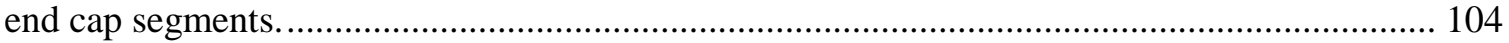


Figure 5-18 Expansion of alternate stent design I: a four-coil helical stent with axial links 106

Figure 5-19 Expansion of alternate stent design II: helical stent design with three sequentially connected two-coil sections (totaling six-coils). 107

Figure 5-20 Expansion of alternate stent design III: helical stent with three sequentially connected three-coil sections (totaling nine coils). 108

Figure 5-21 Expansion of a five-coil stent with a tri-folded balloon using a progressive pressurization pattern. 109

Figure 5-22 Equivalent plastic strain distribution for a $3 \mathrm{~mm}$ radial expansion using the progressive balloon expansion approach.

Figure 5-23 Circumferential stress distribution (GPa) in a helical stent expanded to $3 \mathrm{~mm}$ diameter (without the surrounding artery).

Figure 5-24 Axial stress distribution (GPa) in a helical stent expanded to $3 \mathrm{~mm}$ diameter (without the surrounding artery).

Figure 5-25 Radial stress distribution (GPa) in a helical stent expanded to $3 \mathrm{~mm}$ diameter (without the surrounding artery).

Figure 5-26 Coefficient of friction versus dog boning. .

Figure 5-27 Expansion normal force versus stent diameter.

Figure 6-1 Hyperelastic material model verification based on experimental results for human LAD arteries from implementation of hyperelastic material models [Yang, 2009] and human cellular plaque buildup [Loree, 1994] and implemented in a one-element FE model................. 121

Figure 6-2 Mesh convergence model: a cylinder under axial stretch loading ............................ 123

Figure 6-3 Mesh convergence for artery elements based on von Mises stress (MPa)................. 124

Figure 6-4 Mesh convergence for artery elements based on effective strain ............................ 124

Figure 6-5 Mesh convergence for plaque elements based on von Mises stress (MPa) ............... 125

Figure 6-6 Mesh convergence for plaque elements based on effective strain ............................ 125

Figure 6-7 Geometry of the angioplasty model without plaque buildup.................................. 127

Figure 6-8 von Mises stress distribution (MPa) after axial stretch.......................................... 128

Figure 6-9 Expanded geometry of the angioplasty model without plaque buildup. The two overstretched areas are marked with circles.

Figure 6-10 von Mises stress distribution (MPa) in the arterial wall during full balloon expansion (note that due to the model's symmetry, only half of the artery is shown) 130 
Figure 6-11 Mesh densities and the geometry of artery and plaque parts used in the angioplasty and stenting models

Figure 6-12 Radial displacement (mm) in the artery and plaque elements after axial stretch .... 132

Figure 6-13 von Mises stress distribution (MPa) in the artery and plaque wall at the end of

balloon expansion in the angioplasty model with axial stretch of $1 \mathrm{~mm}$......

Figure 6-14 von Mises stress distribution (MPa) in the artery and plaque wall at the end of

balloon expansion in the angioplasty model without axial stretch

Figure 6-15 Effective strain distribution in the artery and plaque wall at the end of balloon

expansion in the angioplasty model without axial stretch.....

Figure 6-16 Maximum outer radius of the artery during expansion in the angioplasty model with

and without axial stretch.

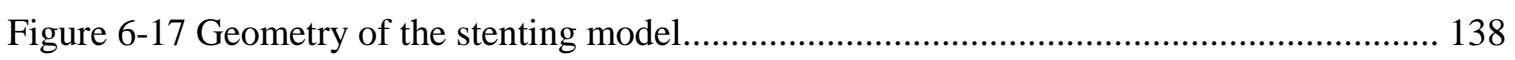

Figure 6-18 the ratio of hourglass and internal energy in the stenting model ............................ 140

Figure 6-19 von Mises stress distribution (MPa) of the artery and plaque before and after full stent expansion.

Figure 6-20 Effective strain distribution at balloon full expansion in artery and plaque

Figure 6-21 von Mises stress (MPa) in the stent at full expansion

Figure 6-22 Foreshortening of the stent

Figure 6-23 Potential injury areas on the plaque buildup following full expansion (shown in red)

Figure 6-24 Artery volume at maximum balloon expansion in different stretch ranges (blue part: low stretch, green part: high stretch and red part: beyond the failure point measured in the experiment).

Figure A- 1 The FEM model of helical stent flexibility.

Figure B- 1 Mesh and geometry of the stent and expansion cylinder. 153

Figure B- 2 Comparison of stent expansion response using implicit and explicit finite element solvers. 155 


\section{List of Tables}

Table 2-1 Summary of the published studies on the material properties of human coronary arteries

Table 3-1 Summary of tests conducted to examine the effect of temperature, immersion in water, and pre-conditioning on the mechanical properties of PLLA and PLGA. ................................... 36

Table 3-2 Average properties of PLLA films based on a sample size of five.............................. 39

Table 4-1 Stress and plastic strain definition for the constitutive model. ................................... 51

Table 4-2 Parametric study results for helical stent uniform expansion. …................................. 72

Table 6-1 Hyperelastic model constants and corresponding $\mathrm{R}^{2}$ value calculated for human artery

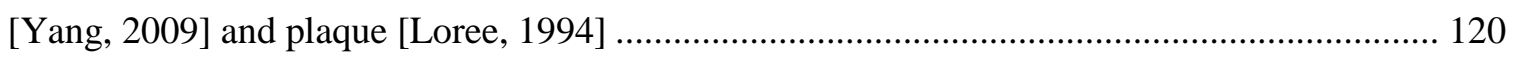




\section{Chapter 1 \\ Introduction}

\subsection{Introduction}

Coronary artery disease is a buildup of fat and cholesterol, also called plaque, in the main arteries that supply blood to the heart, restricting blood flow as these vital passageways gradually narrow and, in some cases, become completely obstructed. This disease claims more than 7 million lives worldwide every year [Claes, 2010], indicating a clear need for effective treatment methods. While several medications are available to treat and reduce symptoms of coronary artery disease, bypass surgery, angioplasty, and stenting are the main interventions for more severe cases. The global market for minimally invasive surgical (MIS) devices and instruments such as stents was estimated at $\$ 13.4$ billion in 2010, with cardiothoracic surgery applications representing $68 \%$ of the total market [BCC research]. Stenting is now a standard MIS method used to restore blood flow in narrowed and blocked arteries. A stent is a tube-like scaffold that can be dilated using a balloon (balloon-expandable) or its own power (selfexpandable) to achieve a customized fit within narrowed arteries following plaque removal, where it serves as a critical support against collapse of these weakened tissues. The vast majority of stents used in MIS interventions are balloon expandable [Martin, 2013] wire mesh designs, rising steadily over the last decade from $70 \%$ of applications reported in 2002 [Stoeckel, 2002].

During surgical deployment, balloon-expandable stents are inserted into a remote location in the artery tree and moved through the artery to the narrowed site. A folded angioplasty balloon is then inflated to expand the stent. Once the stent reaches the required diameter, the balloon is deflated and the plastically-deformed stent remains anchored in the artery. Recent advancements in design and delivery methods have enabled stenting applications to address narrower and more complex lesions [Kandzari, 2002].

First generation stents were commonly fabricated from bare metals such as stainless steel. However, despite early successes, bare metal (BM) stents carry a 30-40\% risk of in-stent immediate restenosis or re-narrowing of the artery [Burt, 2006; Rosanio, 1999; Regar, 2001; Schwartz, 2002]. In these cases, immediate restenosis is primarily caused by blood vessel wall damage sustained during the stent insertion procedure, triggering thrombosis or blood clot 
formation at the site of the damage. This is usually accompanied by an inflammatory response from the immune system and is often treated by administering anti-platelet drugs immediately following surgery to reduce the chance of thrombosis. It has subsequently been found that drug eluting (DE) stents, i.e. stents with a polymeric drug eluting coating, could reduce the risk of restenosis to approximately $10 \%$. Nevertheless, there is still a risk of late restenosis (Neointimal hyperplasia or NIHA) from the proliferation of cells in the intima (smooth muscle wall in the vessel), which can occur 6-12 months after stent insertion. Reports indicate late restenosis is typically the result of artery injury induced by stent implantation followed by foreign body reactions to the stent. To address this, biodegradable drug-eluting (BDE) stents that dissolve gradually while facilitating controlled release of drugs, such as immunosuppressants, tissue growth or inhibitory factors, and antithrombins, are gaining attention as promising alternatives to permanent devices [Regar, 2001; Schwartz, 2002; Wang; 2004].

\subsection{Motivation}

Biodegradable polymers used in stenting applications are limited to drug delivery and loadcarrying structural polymers such as poly (L-lactide-CO-glycolic acid) PLGA and poly-L-lactic acid (PLLA). Helical stent designs are ideal for devices made from biodegradable polymeric materials since the geometry can be readily manufactured and the resulting expanded stent is structurally stable. Another key benefit of this design is ease of deployment; that is, helical stents can bend around small radii that are commonly encountered in arteries and, therefore, have limited free-end contact with the artery, which minimizes potential injury. However, while helical coil polymeric stents may provide a feasible alternative to traditional metallic stents, additional research is required to understand deployment, expansion and fixation of these devices.

Most studies in the literature focus on common wire mesh stents, where inflation of a balloon expands the stent mesh and plastic deformation at the mesh corners locks the stent in the expanded state. In contrast, helical stents undergo larger scale motion as they uncoil and expand. Therefore, available knowledge on the mechanical performance of wire mesh stents cannot be applied to helical stent design. Separate studies must be performed to understand the mechanism of helical stent expansion and determine influential factors to optimize stent expansion and deployment. 
Stenting is a mechanical solution for a biological problem involving large strains, nonlinear materials, and contact between the stent, folded balloon and live tissues (e.g., plaque and artery). Analyses on stent parameters can be performed both experimentally and computationally. Although experimental studies (either in vitro or in vivo) are thought to provide more realistic results, they cannot address the full range of important parameters and are both difficult and costly to conduct. Computational studies, particularly finite element modeling (FEM) efforts, are helpful in developing new designs as they are less complex and expensive than experimental analyses.

In FEM studies, boundary conditions (especially effects attributed to the expanding balloon) must be modeled with care. Several approaches have been applied to model the effect of the balloon on stent expansion, such as creating idealized models that ignore the balloon's complex geometry during expansion and instead consider a fixed internal pressure load [Bedoya, 2006; Rogers, 1999; Dumoulin, 2002; McGarry, 2004; Paszenda, 2005; Mori, 2005; Martin, 2002] or an expanding high stiffness cylinder [Li, 2009; Takashim, 2007; Wu, 2007; Hall, 2006], which depict uniform expansion. Models have also been built to capture the behaviour of an actual folded balloon [Oberhofer, 2006; Gervaso, 2008; De Beulea, 2008; Wu, 2010; Zahedmanesh, 2010]; however, while highly accurate, this approach is computationally expensive. Selection of an appropriate numerical approach and computational platform is often based on model complexity and efficiency requirements, where tradeoffs between accuracy and computational costs must be considered.

\subsection{Problem statement}

Despite the advantages helical stents provide for biodegradable stent applications, data and research supporting their applicability for real-world surgical deployment is limited. What we do know from our limited understanding of helical stenting is that this design suffers from high foreshortening. In addition, helical stents often experience non-uniform local expansion (dog boning), which can prohibit full expansion using conventional methods. Finite element analysis of the actual expansion boundary conditions using a folded balloon coupled to a helical stent, which gradually expands the balloon, can accurately predict transient deformation during expansion including the 'dog bone' shape. However, modeling balloon-stent expansion requires extensive computational resources due to the nonlinearities and complexities of the contact interfaces. 
Basic expansion models (such as uniform expansion) can be used to evaluate and optimize expansion factors, such as foreshortening. However, as a strong connection has been reported between artery injuries induced during stent deployment and in-stent restenosis (ISR) risk, FEM can be invaluable to determining the factors that directly influence successful stent deployment.

The thesis project described herein is part of a wider research activity initiated by Nanyang Technological University (NTU) [Venkatraman, 2006A]. Our NTU collaborators developed the design and deployment conditions of a drug-eluting biodegradable helical stent used in a MIS application. In the present study, a FE model of a bilayer helical coil stent consisting of PLLA and PLGA was built to evaluate performance using finite element analysis. In vitro material characterization studies showed that a pre-insertion water-soaking step that mimics body implantation conditions provided the required ductility level for expansion. In this case, the mechanical contribution of the outer PLGA layer was negligible since it softened significantly under environmental conditions. The viscoelastic response was not considered in this study because the strain rate during expansion was relatively slow and the material response was primarily plastic.

The helical stent geometry investigated in the present study was expanded from an inside diameter of $1 \mathrm{~mm}$ to a final diameter of $3 \mathrm{~mm}$ using three different numerical expansion methods: (I) displacement-driven, (II) uniform expansion cylinder, and (III) folded balloon expansion. It should be noted that, in cases where full expansion to a $3 \mathrm{~mm}$ diameter was not achieved, this was due to correct prediction of physical response (e.g., coil interaction), which led to locally high deformations or stresses and, subsequently, to earlier termination of the calculation. However, this was useful information in that we could investigate various expansion methods and identify specific aspects of non-uniform expansion that are not desirable for actual stent designs.

Stent deployment was studied using the progressive expansion pattern in order to: i) evaluate the effects the presence of the artery has on helical stent expansion, and ii) find the stress distribution on the artery during helical stent expansion.

Figure 1-1 presents a flowchart of the research described herein. 


\subsection{Thesis outline}

Chapter 2 provides a brief review of recent advances in stent design, outlines remaining technological challenges facing current stent devices, and discusses the role helical stents will play in resolving these challenges. Shortcomings and lack of research data related to helical stent expansion are specifically underscored. In this chapter, the role FEM may play in determining and possibly resolving the helical stent expansion problem is briefly described. Finite element code formulations (both implicit and explicit) and the applications of each code are reviewed. An in vitro study on PLLA and PLGA that provides both tensile and creep (time dependent) experimental results is described in Chapter 3. Implementations of the three main FEM approaches in helical stent expansion (expansion with inner pressure, high stiffness, and folded balloon inflation) are studied in Chapter 4. Chapter 5 discusses factors that influence the "dog boning" pattern of helical stent expansion and offers potential methods to resolve this issue. The mechanical results from one of these methods (progressive expansion pattern) are also analyzed in the chapter. In Chapter 6, helical stent expansion is studied using the progressive expansion pattern method during deployment inside an idealized artery with plaque buildup to determine the effect of the surrounding artery on stent expansion and, conversely, the effect of the stent's expansion on the artery wall. In the seventh and final chapter, conclusions are drawn from research described in earlier chapters. Limitations of the study and potential future research directions are also discussed. 


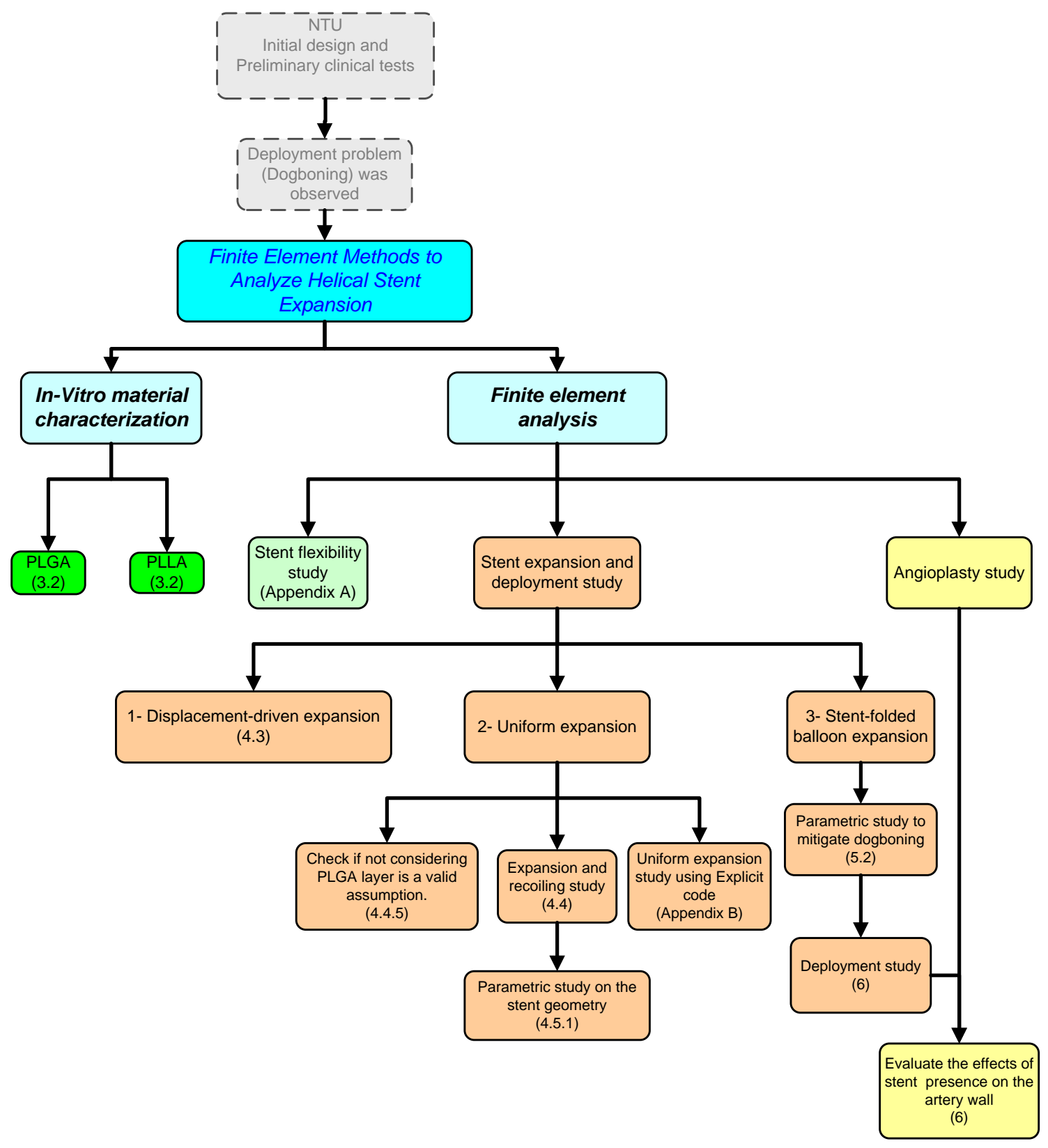

Figure 1-1 Research activity flowchart. 


\section{Chapter 2 \\ Background}

Finite element modeling techniques are increasingly used to build patient-specific tools with strong potential to aid in diagnosis, provide decision-support, and predict clinical outcomes of new treatment and surgical interventions prior to in vivo implementation [Holzapfel, 2005; Kural, 2012]. Reliability remains a key challenge to the clinical application of these integrative tools for cardiac stenting procedures; however, the first step towards addressing this issue is the implementation of highly accurate material and geometry properties into the code.

Cardiac stents are tube-like scaffolds used to support narrowed arteries following plaque removal and arterial widening procedures. Although stents may be self-expandable, the vast majority of stents used today are balloon-expandable. During deployment, the stent is positioned inside the narrowed part of the artery, and then gradually expanded to a nominal diameter by inflating a folded angioplasty balloon set inside the stent. The balloon is then deflated and removed while the plastically-deformed stent remains anchored in the artery.

First generation stents were bare metal (BM) devices that carried a 30-40 \% risk of immediate restenosis [Burt, 2006; Rosanio, 1999; Regar, 2001; Schwartz, 2002]. The introduction of localized drug delivery through drug eluting (DE) stents, i.e. stents with a polymeric drug eluting coating, has reduced the risk of short-term restenosis to approximately $10 \%$. Nevertheless, the risk of late restenosis, which occurs 6-12 months after stent insertion, remains high. It has been reported that late in-stent restenosis (ISR) is associated with injuries to the artery induced during stent deployment, which is followed by foreign body reactions (FBR) to the stent. Two approaches have been applied to address ISR. The first seeks to optimize both stent deployment and inflation techniques in order to minimize high stress areas on the artery wall [Chua, 2004; Lally, 2005; Liang, 2005; Bedoya, 2006; Gervaso, 2008; Gijsen, 2008], while the second employs biodegradable drug-eluting (BDE) stents to eliminate FBR. BDE stents that dissolve gradually while releasing various drugs, such as anti-inflammatory, anti-thrombin medications, are gaining attention as promising alternatives to conventional BM stent devices [Regar, 2001; Schwartz, 2002; Wang, 2004]. Materials that can be used in biodegradable stents must be biocompatible [Lally, 2005] and balloon-expandable stents should demonstrate appropriate mechanical properties to support the artery and undergo plastic deformation during deployment 
[Lally, 2005]. Potential candidate materials for BDE stents are limited to drug delivery and loadcarrying structural polymers such as poly-L-lactide-CO-glycolic acid (PLGA) and poly-L-lactic acid (PLLA), where PLLA is one of the most widely studied biocompatible materials [Athanasiou, 1996, Elakkiya 2013]. Although current BDE stents may minimize FBRs, they cannot prevent arterial injuries incurred during deployment. Thus, alternative stent design and deployment techniques are required to minimize injuries.

Polymeric stents are not as common as their metallic counterparts and many are still in the pre-clinical design stage. In the literature, two types of biodegradable stent designs have been employed. The first designs were fiber braided [Welch 2008 and Vaajanen 2003] and helical stents [Tan 2001 and Su 2003], which often exhibit low collapse pressure. The second type covers polymeric stent designs that mimic traditional metallic stent devices, such as common wire mesh structures [Grabow 2007 and Grabow 2005]. Although these stents demonstrate improved collapse pressure, they are difficult to fabricate and sterilize [Grabow 2005]. In addition, the stress concentrations associated with wire mesh devices can result in uncontrolled, non-uniform degradation since biodegradable polymers such as PLLA [Soares 2010] and PLGA [Chu 1985] exhibit higher degradation rates at highly strained regions.

\subsection{Helical stents}

Helical stent geometries are easy to process, which makes them ideal for biodegradable polymer-based devices (Figure 2-1). During helical stent processing, a polymer film is spun around a cylindrical mandrel to obtain a coil of controllable thickness. Resulting devices can be built to have high flexibility, enabling insertion in curved passages [Lansky 2000]. The stent-toartery ratio, representing the stent coverage, can also be controlled by the width of the stent. In addition, by comparison with common slotted and wire mesh stents, which have sharp edges, helical stents may reduce potential injury sites upon insertion and subsequently reduce the incidence of restenosis [Bedoya, 2006; Gervaso, 2008]. 


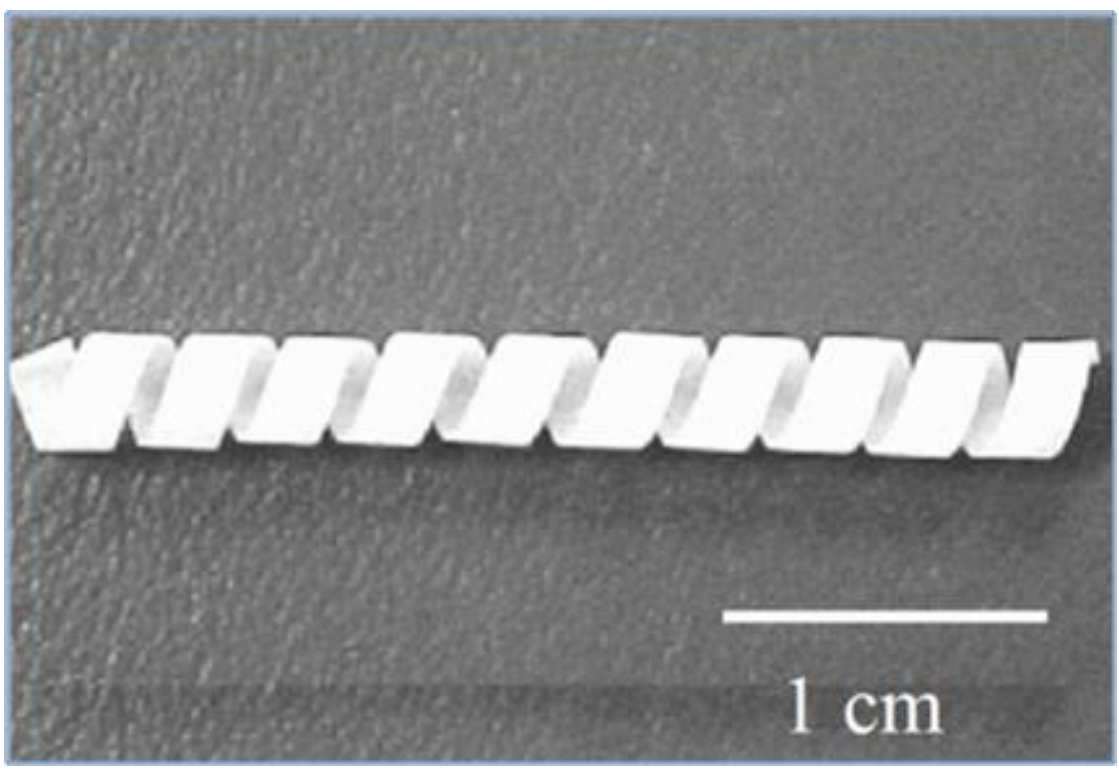

Figure 2-1 A typical helical stent [Venkatraman, 2003].

Beyond some clinical reports [Vaajanen, 2003; Tamura, 1996; Vogt, 2004; Meng, 2006; Venkatraman, 2003], very few studies have been undertaken to evaluate mechanical performance and design of helical stents. It has been found that a typical PLLA-based helical stent has a collapse pressure of 2.5 bar. Stents should generally demonstrate a collapse pressure of 0.4 bar for coronary arterial applications [Agrawal, 1992]. Furthermore, the biodegradable characteristics of the stent do not affect its radial strength over time [Venkatraman, 2003; Venkatraman, 2006B]. It has also been reported that helical stents suffer from large non-uniform expansion, termed 'dog boning', which can affect expansion and therefore function (Figure 2-2); however, the associated causes of this behaviour remain unclear. This is primarily due to challenges in experimental testing where only limited measures of response are available, and varying material properties and geometry is time consuming and expensive to implement. 

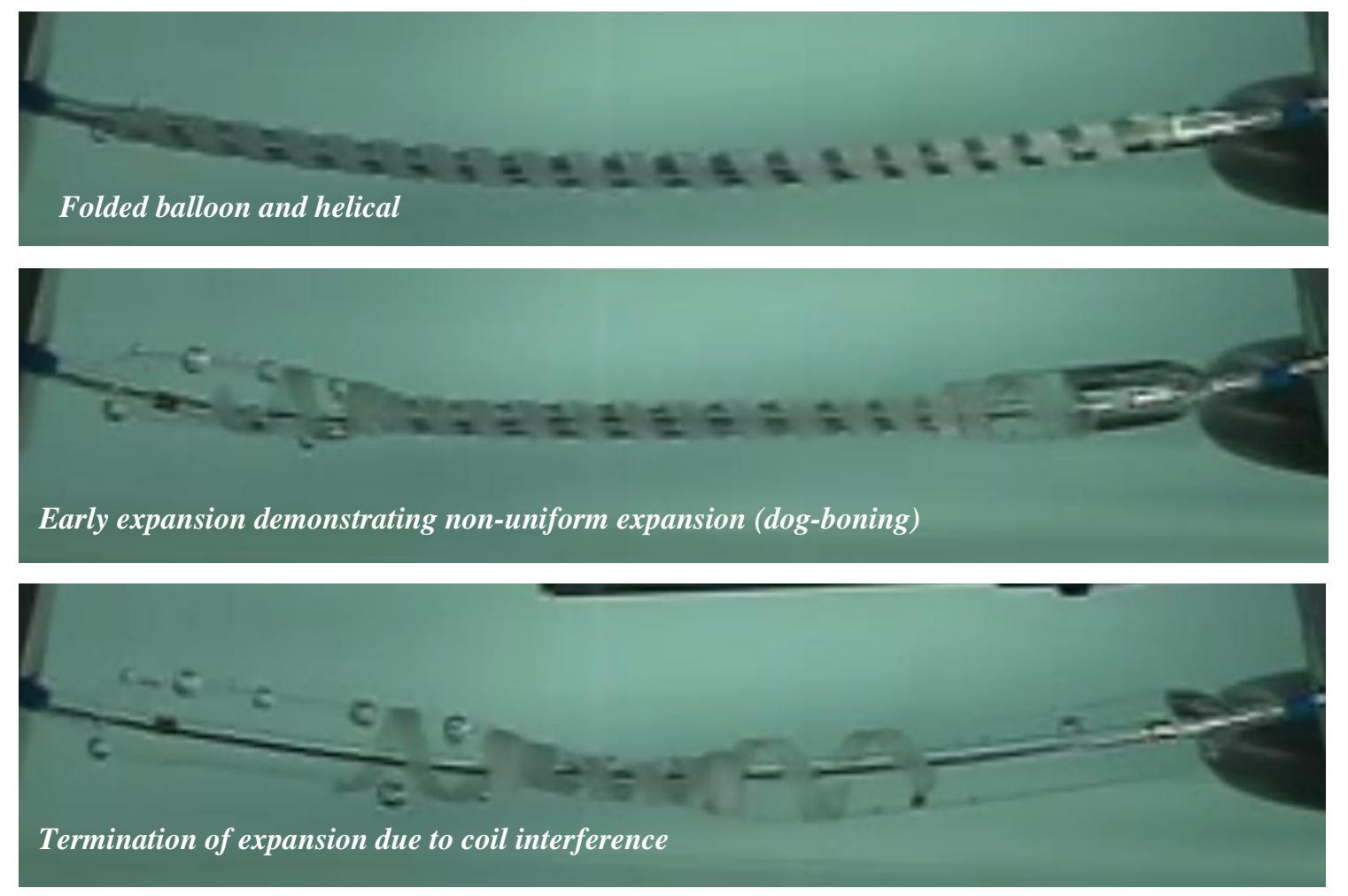

Figure 2-2 Balloon expansion of a helical stent in saline bath, demonstrating 'dog boning' and interference of adjacent coils.

Wire mesh stents typically expand via plastic deformation (or bending) of the struts, a mechanism that is both widely studied and well documented [De Beulea, 2008]. In contrast, experimental tests indicate that helical stent expansion involves larger scale motion as the stent uncoils which has not been extensively studied in the literature.

\subsection{Stent Expansion and Recoiling}

After expanding the stent to the required diameter, the balloon is deflated and the plasticallydeformed stent remains anchored in the artery. Once the balloon is removed, stents tend to reduce in both radius and length; that is, they undergo radial and longitudinal recoiling, respectively. One goal of deployment studies is to ensure the stent is positioned properly in the artery or, for biodegradable materials, remains in place until it dissolves.

Generally, a stent's mechanical performance is measured against various evaluation parameters including foreshortening, dog boning, and radial or axial recoiling. Minimizing all of 
these parameters is the goal of stent design. Foreshortening is a measure of the change in stent length during expansion; dog boning measures end-first expansion of the stent; and axial and radial recoiling measure the change in radius and length of the stent following deflation of the balloon.

Foreshortening affects the stent's position in the artery and is therefore considered very important to determining the therapeutic efficacy of stent design. In addition, relative movement of the stent in the artery during expansion can cause frictional effects that may in turn cause injuries to the delicate inner layer of the artery [Wang, 2006].

Foreshortening is defined as [Migliavacca, 2002]:

Foreshortening $=\frac{L_{1}-L_{2}}{L_{1}} \times 100$

Equation 2-1

where $\mathrm{L}_{1}$ is the original length and $\mathrm{L}_{2}$ is the final length.

Traditionally, recoiling has been measured as:

Radial recoiling $=\frac{R_{2}-R_{3}}{R_{3}} \times 100$

Equation 2-2

where $R_{2}$ is the expanded radius and $R_{3}$ is the final radius.

Dog boning measures the end first expansion of stents [Migliavacca, 2002]:

Dog boning $=\frac{D_{\text {Distal }}-D_{\text {Central }}}{D_{\text {Distal }}} \times 100$

Equation 2-3

where $\mathrm{D}_{\text {Distal }}$ is the diameter of the end of stent and $\mathrm{D}_{\text {Central }}$ is the diameter of the middle of stent.

\subsection{FEM studies on stenting}

Stent expansion is a complex mechanical procedure involving large strains, nonlinear materials, and contact between the stent, folded balloon and live tissues (e.g., plaque and artery). In the literature, mechanistic analyses of this procedure have included experimental and computational studies. Although experimental studies (in vitro or in vivo) are considered to provide more realistic results than simulation studies, they cannot address all relevant performance parameters. Furthermore, since experimental studies are both difficult and costly to conduct, they are usually undertaken at later stages of development or to validate computer simulation predictions. Computational studies, particularly finite element modeling (FEM), are helpful in developing new designs and minimizing experimental cost [Stolpmann, 2003; 
Auricchio, 2000; Wang, 2006; Rogers, 1999; Migliavacca, 2002; Walke, 2005]. However, due to computational limitations, finite element models are generally simplified to analyze only the most important parameters. It is generally accepted that even first order analyses are extremely useful for parametric studies that evaluate the influence of various factors, such as geometry and mechanical properties, on the success of stenting treatment, particularly if some form of experimental work can verify the results.

Helical stents often experience non-uniform local expansion (dog boning), which can prohibit full dilation of the stent using conventional methods. Development of viable stent designs and deployment methodologies is challenging and can be supported by numerical analysis; however, this complex problem is often approached with simplified boundary conditions that may not be appropriate for helical stents.

In general, the primary differentiator between numerical studies recently undertaken to investigate stent expansion is the computational method used to predict that behaviour, which dictates the computation time and model complexity. Indeed, a variety of computational approaches, each with varying levels of complexity, have been applied to investigate various expansion methods for (primarily) wire mesh stent designs, and typically consider simple uniform pressure or displacement on the inner surface of the stent [Dumoulin, 2000; Auricchio, 2001; Etavea, 2001; Migliavacca, 2002; Liang, 2005; Migliavacca, 2005; Oberhofer, 2006; Early, 2008; De Beulea, 2008; Zahedmanesh, 2009; Pericevic, 2009; Kiousis, 2009; Zahedmanesh, 2010].

The simplest possible stent expansion FEM strategy is to apply inner pressure/displacement to the inner surface of the stent [Pericevic, 2009; Auricchio, 2001; Etave, 2001; Migliavacca, 2002; Dumoulin, 2000; Early, 2008; Migliavacca, 2005; Zahedmanesh, 2009]. A study by De Beule et al [De Beule, 2008] showed that, while the final and pressure-diameter measures predicted by this method resemble the experimental results, it does not effectively simulate the profile of the stent during expansion.

Stent expansion has also been investigated using a high stiffness cylinder, which generates uniform radial expansion [Li, 2009; Takashim, 2007; Wu, 2007; Hall, 2006]. However, any nonuniform aspects of expansion related to stent geometry (i.e., dog boning) cannot be predicted with this method. It has been noted that, although the transitional behaviour cannot be predicted, the final shape of the stent produced using the high stiffness cylinder is the same as the balloon 
expanded stent [De Beulea, 2008]. Nevertheless, load path dependence can be important to determining the plastic deformation required to expand the stent.

A flexible cylinder has also been applied to expand the stent in an effort to further simplify computational complexity [Liang, 2005; Kiousis, 2009]. In this case, tuning or adjustment of the cylinder properties was found to produce reasonable behaviour; however, once developed for a particular scenario, this method is not necessarily applicable to other load cases or stent designs since the properties are often adjusted on a case-by-case basis [De Beulea, 2008].

Coupling a folded balloon model to the stent and gradually expanding the balloon to predict the transient deformation during expansion, including the 'dog bone' shape, provides the most accurate representation of the actual expansion boundary conditions. However, modeling balloon/stent expansion requires extensive computational resources due to the nonlinearities and complexities of the contact interfaces.

Several different methods have been considered to create the initial folded balloon, including CT-imaging of an actual folded balloon [Gervaso, 2008; De Beulea, 2008] and extruding an assumed balloon cross-section in the axial direction [Wu, 2010]. In cases where the balloon end caps are not included, an applied axial stress was required to compensate for the effect of the balloon end conditions. A more accurate model includes a simulation to initially fold the balloon [Zahedmanesh, 2010; Oberhofer, 2006], followed by integration with a stent, and finally expansion of the balloon. No matter how the folded balloon geometry is developed, stent expansion is a computationally expensive model to run; however, computationally accelerated techniques, such as parallel computing, can be used to solve this problem.

The choice of stent expansion technique is always the center of debate in wire mesh FEM studies; in fact, several studies have specifically sought to address this issue [Pericevic, 2009; Auricchio, 2001; Etave, 2001; Migliavacca, 2002; De Beule, 2008; Liang, 2005; Kiousis, 2009; Zahedmanesh, 2010; Oberhofer, 2006]. However, very few studies have considered new designs, such as helical stent devices. Part of the present study is dedicated to comparing the applicability and results of the three most commonly employed wire mesh stent expansion methods applied to the helical stent. During this study, the mechanism of helical stent expansion is described and a solution to overcome the dog boning issue is provided. 


\subsection{Stent expansion inside the artery}

Clinical reports indicate that there is a strong connection between artery injuries incurred during stent deployment and risk of in-stent restenosis (ISR) [Gu 2010]. Several studies have used FE modeling to determine the factors that influence successful stent deployment, with particular emphasis on stent expansion inside the artery [Auricchio, 2001; Berry, 2002; Prendergast, 2003; Chua, 2004; Miglivacca, 2004; Holzapfel, 2005; Lally, 2005; Liang, 2005; Walke, 2005; Ballyk, 2006; Bedoya, 2006; Marrey, 2006; Kiousis, 2007; Takashim, 2007; Timmins, 2007; Wu, 2007; Gervaso, 2008; Gijsen, 2008; Capelli, 2009; Early, 2008; Pericevic, 2009]. Thus far, all deployment studies have focused on metallic wire mesh stents inside both normal and occluded coronary arteries, with the earliest studies primarily targeting a common Palmaz-Schatz wire mesh stent design [Auricchio, 2001; Chua, 2004]. Later modeling efforts addressed new stent designs [Bedoya, 2006; Wu, 2007], including those made of stainless steel (either 316 L [Auricchio, 2001; Lally, 2005; Liang, 2005; Holzapfel, 2005; Bedoya, 2006; Wu, 2007, Gervaso; 2008, Gijsen, 2008; Capelli, 2009] or 304 [Chua, 2004]) and MP35N cobalt alloy [Pericevic, 2009]. In most cases, the artery (and plaque) was modeled as hyperelastic material [Auricchio, 2001; Lally, 2005; Liang, 2005; Holzapfel, 2005; Bedoya, 2006; Wu, 2007; Gervaso, 2008; Gijsen, 2008; Capelli, 2009; Pericevic, 2009] where the material model

constants were derived by fitting the material model curve to in vitro experimental data [Hayashi, 1997; Veress, 2000; Loree, 1994; Prendergast, 2003; Holzapfel, 2000; Holzapfel, 2005; Lally, 2004]. The geometry of the artery was mostly modeled as an idealized cylindrical shape [Auricchio, 2001; Chua, 2004; Lally, 2005; Liang, 2005; Bedoya, 2006; Wu, 2007; Capelli, 2009; Pericevic, 2009]. Actual arteries have three tissue layers [Pizlo, 2001]; thus, studies have either modeled the effective structural layer or a composite of all three layers [Gervaso, 2008; Capelli, 2009]. In some cases, a patient's specific artery geometry was imported from the results of magnetic response imaging (MRI) [Holzapfel, 2005; Gijsen, 2008].

The early studies concluded that it is possible to obtain a validated stent deployment model that can evaluate mechanical behaviour of the stent [Auricchio, 2001], where high stress areas on the artery (or plaque) coincide with experimentally observed injury areas [Chua, 2004] and a correlation can be found between the predicted volume of the arterial wall under high stress and the reported ISR rate caused by arterial injury during stent deployment [Lally, 2005; Liang, 2005]. In an effort to minimize the occurrence of high stress areas, recent studies have employed 
FE models to evaluate the relationship between stent design specifications and potential injury sites [Bedoya, 2006; Gijsen, 2008; Capelli, 2009]. More recent deployment studies have taken advantage of advances in robust high efficiency numerical methods to include more realistic expansion conditions, such as stent expansion with tapered balloon inflation [Capelli, 2009] that takes into account patient-specific conditions such as artery geometry and plaque buildup [Gijsen, 2008]. Despite these advances, helical and polymeric stent deployment has not been addressed to date, and is thus a key focus of this study.

\subsection{Implicit versus explicit FEM}

FEM-based mechanical analyses can provide insight into the expansion and performance of stent designs, as well as highlight critical or sensitive parameters. More important, model validation results against appropriate experimental data may be sensitive to the model boundary conditions. FEM studies on stenting have employed both implicit [Welch, 2008; Migliavacca, 2007; Migliavacca, 2002, Lia; 2009; Lim, 2008] and explicit codes [Lim, 2008; Gervaso, 2008; Ju, 2008; De Beulea, 2008; Zahedmanesh, 2009; Mortier, 2009; Li, 2009; Pericevic, 2009; Mortier, 2008]. However, despite increasing use of FEM in stenting analysis there is no established methodology for choosing one method over another, or for defining suitable parameters that facilitate the analysis and do not affect the final results. Selection of a numerical approach and computational platform are often based on model complexity and requirements.

Implicit finite element methods are ideal for low deformation rate processes, when the contact interfaces are simple. These approaches provide reasonable calculation times since large time steps are acceptable in quasi-static or low rate processes, and are therefore often used to predict simple expansion methods.

In general, contact issues limit the use of implicit approaches. Explicit (time domain) methods, which employ small time steps to accurately follow the model, are required to solve more complex, nonlinear modeling problems, such as material and geometrical nonlinearities, or the complicated contact interface between the balloon and stent. However, while small stable time steps enable advanced modeling of complex behaviours, they are also the primary drawback of the explicit method. These time steps are dependent on the size of the elements and elastic wave speed in the materials. For typical material properties and mesh sizes, stable time steps may be on the order of microseconds, requiring upwards of $10^{8}$ calculation cycles to 
address full expansion. In certain cases, this limitation can be reduced through mass or time scaling. Therefore, selection of an appropriate modeling approach is very important and must be considered with respect to the complexity of the model. Although simplified approaches allow for more simulations and larger parametric studies, limitations with respect to boundary conditions and associated assumptions may render them inappropriate for highly nonlinear problems. For advanced models containing complex contact interfaces, material nonlinearities, and large deformations, explicit code is more attractive. Moreover, the contact algorithm in explicit code is more straightforward than would be achieved with implicit formulations, which makes it the first choice when analyzing complex contacts.

\subsubsection{Implicit formulations}

Implicit and explicit formulations are both methods of solving equations that explain geometrical (i.e., large deformations) and/or material nonlinearities (e.g., displacement or time dependent properties in hyperelastic and viscoelastic materials).

In stiffness-based implicit methods, the incremental strategy is based on a full Newton iterative method [Chung, 1998]. The dynamic quantities at time $\mathrm{t}+\Delta \mathrm{t}$ are solved based on the values at time $\mathrm{t}$ and time $\mathrm{t}+\Delta \mathrm{t}$, implicitly:

$\Delta u_{i+1}=\Delta u_{i}+K_{t}^{-1} \cdot\left(F_{i}-I_{i}\right)$

Equation 2-4

where $\Delta \mathrm{u}, \mathrm{K}_{\mathrm{t}}, \mathrm{F}$, and I are the increment of displacement, the current tangent stiffness matrix, the applied load vector, and the internal force vector, respectively.

The algorithm for an implicit dynamic procedure is defined as follows:

$M \ddot{u}_{i+1}+(1+\alpha) K u_{i+1}-\alpha K u_{i}=F_{i+1}$

Equation 2-5

where $\mathrm{M}, \mathrm{K}, \mathrm{F}$, and $\mathrm{u}$ are the mass matrix, stiffness matrix, applied load vector and displacement vector, respectively.

The displacement and velocity vectors are calculated as follows:

$u_{i+1}=u_{i}+\Delta t \dot{u}_{i}+\Delta t^{2}\left(\left(\frac{1}{2}-\beta\right) \ddot{u}_{i}+\beta \ddot{u}_{i+1}\right)$

Equation 2-6

and

$\dot{u}_{i+1}=\dot{u}_{i}+\Delta t\left((1-\gamma) \ddot{u}_{i}+\gamma \ddot{u}_{i+1}\right)$

Equation 2-7 
Although implicit code is an unconditionally stable procedure, implicit equations should be solved for each time increment. While this method is efficient for small and simple models, modeling complicated problems (e.g., large contact interfaces and material nonlinearity) results in very high run times, representing a major drawback of this approach. That is, the implicit approach requires shortened time increments to model complex contact interfaces, and, since a tangent stiffness matrix must be calculated for each increment, computation time is dramatically increased. Furthermore, small increments can cause divergence and, in complicated models, the Jacobian matrix is hard to calculate due to local instabilities.

Adding contact constraints to implicit code dramatically increases the complexity of the resulting model, but does not increase the number of equations required.

\subsubsection{Explicit Formulations}

Explicit code was originally developed to address the computation time issue inherent to the implicit method. In explicit schemes, the central difference operator is commonly used for stress analysis:

$\ddot{u}_{i}=M^{-1} \cdot\left(F_{i}-I_{i}\right)$

Equation 2-8

$\dot{u}_{1+\frac{1}{2}}=\dot{u}_{i-\frac{1}{2}}+\frac{1}{2}\left(\Delta t_{i+1}+\Delta t_{i}\right) \ddot{u}_{i}$

Equation 2-9

where M , F, and I are the mass "lumped" or diagonal matrix, the applied load vector and the internal force vector, respectively.

Combining explicit equations with a diagonal mass matrix is a key advantage of this method, minimizing the solution cost for each time increment. However, it is conditionally stable. To ensure stability, each time increment should be shorter than the time required for an elastic wave to cross the smallest element's dimension in the model. The upper bound of the time increment is as follows:

$\Delta t \leq \frac{2}{\omega_{\max }}$

Equation 2-10

where $\omega_{\max }$ is the maximum eigenvalue of the body. Estimating $\omega_{\max }$ is not an easy task. There is a more conservative value that gives the upper bound of $\Delta \mathrm{t}$, as follows:

$\Delta t=\min \left(\frac{L_{C}}{c_{d}}\right)$

Equation 2-11 
where $\mathrm{L}_{\mathrm{C}}$ and $\mathrm{C}_{\mathrm{d}}$ are the characteristic element dimension and the current effective dilatational wave speed of the material [ABAQUS manual], respectively.

The implicit method requires time increments that are roughly 2 to 3 orders of magnitude larger than those used in the explicit method to solve the same model.

Efficiency gains achieved through explicit integration can be attributed to its non-iterative nature and the use of a diagonal (lumped) mass matrix, as opposed to the tangent stiffness matrix used in implicit integration.

Adding contacts in explicit code changes the model constraints and, consequently, the maximum eigenvalue of the model. Therefore, smaller time increments are required to stabilize explicit code. In this method, the cost of analysis increases with the order of the model itself, rather than the complexity of the model. Therefore, explicit code can more efficiently handle large or complicated models.

The major drawback of the explicit method is the very small time increments used, which drive up the computation time, particularly for small, low density, high stiffness parts.

\subsubsection{Time and mass scaling in explicit calculations}

The explicit method was originally developed to address impact-type and short-time loadings. Stenting, however, is generally performed with low velocity expansion and is, therefore, considered a quasi-static procedure. In order to apply the explicit method to quasi-static models it is customary to undertake the analysis in virtual time using time scaling or mass scaling approaches. Time scaling applies loads in shorter time periods than would be experienced in reality. Run time decreases by the same factor applied to the time scaling. Time scaling cannot be used to expedite models with rate-dependent materials (i.e., where inertia effects are important). In mass scaling, the density is increased. Scaling the density by two orders of magnitude increases the time step by one order. Sometimes, instead of increasing the density (mass) of the entire structure, only the mass of the elements with the smallest dimensions, which often determine the smallest time step during solution, is changed - a method called selective mass scaling.

Care should be taken to evaluate the effect of virtual time and mass scaling, which increases inertial effects and can change the final results [Chung, 1998]. In order to find a suitable scale factor, an energy factor is calculated. 
The potential error induced by mass scaling is expressed as the ratio of kinetic energy to internal energy. Increasing the scale factors increases the kinetic energy of the model. The share of the kinetic energy from the internal energy should be less than 1-5\% for a quasi-static process [Ju, 2008; Zahedmanesh, 2009; Gervaso, 2008; Mortier, 2008].

$e(t)=\frac{E_{K}(t)}{E_{\text {int }}(t)}$

Equation 2-12

where $E_{K}$ and $E_{i n t}$ are kinetic and internal energies. This formula was originally developed for sheet metal forming.

\subsection{Constitutive modeling}

Most structural materials exhibit instantaneous responses to applied loads or displacement, and the resulting stress-strain relationship can be simply described with Hooke's law. Other "viscoelastic" materials, such as polymers, exhibit a combination of both liquid-like (viscous) and solid-like (elastic) properties when subjected to an external load.

\subsubsection{Polymer material models}

When viscoelastic materials are subjected to a constant step load, the strain level increases with decreasing strain rate (Figure 2-3). This behaviour is called creep. If the stress is removed, strain decreases gradually with a decreasing rate, which is referred to as recovery. The stress level required to maintain a constant strain also decreases over time, which is called stress relaxation (Figure 2-4). 


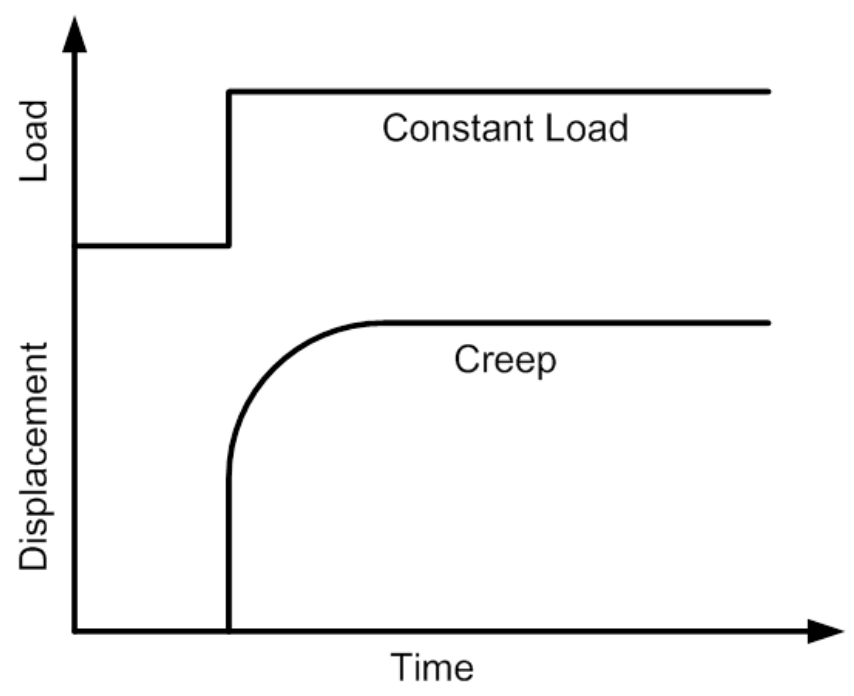

Figure 2-3 Creep curve

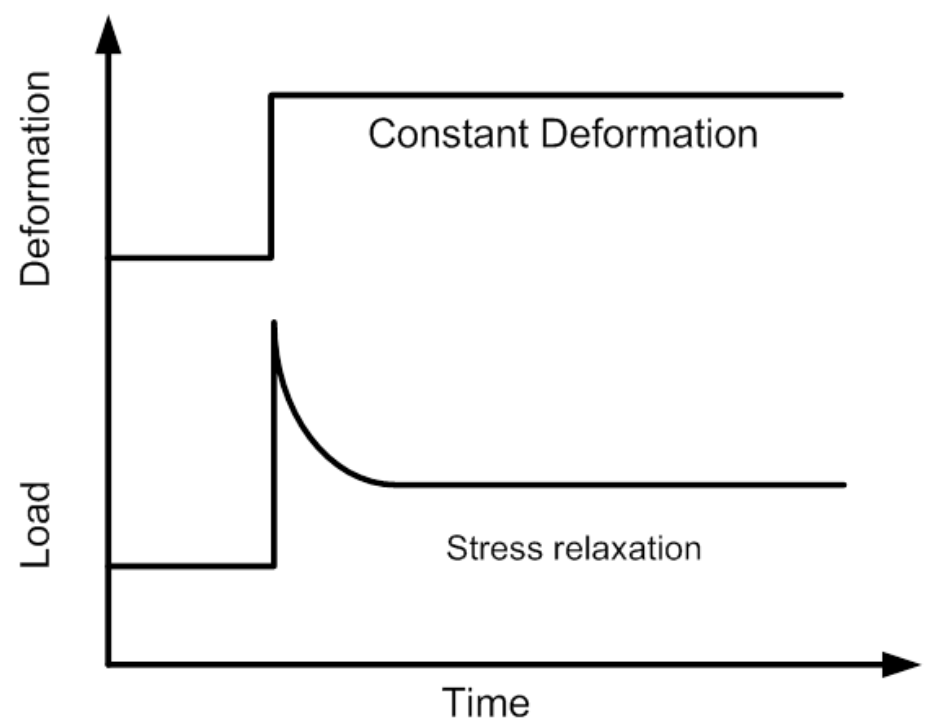

Figure 2-4 Stress relaxation curve

Constitutive models of viscoelastic materials should include elements to describe both elastic (instantaneous) and viscous (time dependent) properties. Basic viscoelastic models use mechanical analogies of spring and dashpot models to describe instantaneous and time dependent properties of viscoelastic materials, respectively (Figure 2-5). The spring model can be described with Hooke's law, while dashpot-type mechanical behaviour can be described as follows 
$\sigma=\eta \frac{d \varepsilon}{d t}$

Equation 2-13

where $\eta$ is viscosity.

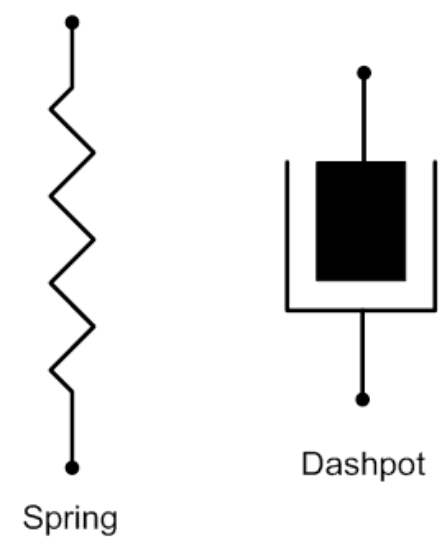

Figure 2-5 Spring and dashpot elements.

Basic viscoelastic models generally combine spring and dashpot elements. A model based on a series of spring and dashpot elements is called a Maxwell model. Its representative differential equation is described as follows

$E \frac{d \varepsilon}{d t}=\frac{d \sigma}{d t}+\frac{\sigma}{\tau_{r}}$

Equation 2-14

where $\tau_{\mathrm{r}}$ is the relaxation time and is equal to $\eta / \mathrm{E}$. This model accurately describes behaviour of viscoelastic fluids and stress-relaxation, but not creep [Ferry, 1970].

\subsubsection{Elastic-plastic properties}

Although polymers have rate dependent properties, in cases where constant strain rate conditions exist (such as in standard stent expansion practice), the strain rate part of the constitutive model can be ignored. Therefore, the classical plasticity theory is true in these cases and the yield point is assumed to be rate independent. The elastic portion of the constitutive model can be defined with Hooke's law since it is much smaller than the plastic portion.

Several failure (yield) theories have been employed to determine when the material starts to yield and deform permanently (plastic strain portion), such as: the maximum normal stress theory (Lame-Navier), maximum shear stress theory (Tresca), and maximum distortion energy 
(or maximum octahedral shear stress) theory (von Mises). It has been shown that, in general, polymers tend to behave based on the von Mises failure theory [Brinson, 2008], which is defined as follows:

$\sigma_{1}^{2}+\sigma_{2}^{2}+\sigma_{3}^{2}-\left(\sigma_{1} \sigma_{2}+\sigma_{2} \sigma_{3}+\sigma_{1} \sigma_{3}\right)=\left.2 \sigma_{f}^{2}\right|_{\text {tensile }}$

\section{Equation 2-15}

where the $\sigma \mathrm{s}$ are principal stresses and $\sigma_{\mathrm{f}}$ is the yield stress limit of the material.

Different methods can be used to find the yield point from experimental data; however, the proportional limit method is widely accepted for polymers [Brinson, 2008].

In addition, temperature dependent properties can be ignored in mechanical modeling (such as FEM) if it is known that the stent is expanded in a constant temperature.

Since polymer material properties are highly process-sensitive, the material properties of both PLLA and PLGA were measured experimentally for this project.

An isotropic elastic-plastic model, which is the most cost effective formulation to model plastic materials [LS Dyna theory manual], was used to forecast plasticity where only the history variable can give the effective plastic stain, which is defined as follows:

$\varepsilon_{e f f}^{p}=\int_{0}^{t} d \varepsilon_{e f f}^{p}$

Equation 2-16

where $d \varepsilon_{e f f}^{p}=\sqrt{\frac{2}{3} d \varepsilon_{i j}^{p} d \varepsilon_{i j}^{p}}$. And the plastic tangent modulus is defined in terms of the input tangent modulus, $\mathrm{E}_{\mathrm{t}}$, as follows

$E_{p}=\frac{E E_{t}}{E-E_{t}}$

Equation 2-17

\subsubsection{Hyperelastic isotropic material model}

A hyperelastic isotropic material model can be described as a strain energy function $\mathrm{W}$, of the left Cauchy-Green tensor B with F being the deformation gradient.

$\mathrm{B}=\mathrm{FF}^{\mathrm{T}}$

Equation 2-18

Cauchy stress $\sigma_{\mathrm{ij}}$ can be given in terms of Cauchy-Green tensor, $\mathrm{B}_{\mathrm{ij}}$, as follows:

$\sigma_{i j}=-p+2 \frac{\partial W}{\partial I_{1}} B_{i j}-2 \frac{\partial W}{\partial I_{2}} B_{i j}^{-1}$

Equation 2-19

where $\mathrm{W}$ is the strain energy function and $\mathrm{I}_{1}, \mathrm{I}_{2}$, and $\mathrm{I}_{3}$ are stretch invariants of $\mathrm{B}_{\mathrm{ij}}$. 
$\mathrm{I}_{1}=\operatorname{tr}(\mathrm{B})=\left(\lambda_{1}\right)^{2}+\left(\lambda_{2}\right)^{2}+\left(\lambda_{3}\right)^{2}$

Equation 2-20

$\mathrm{I}_{2}=\operatorname{tr}[\operatorname{adj}(\mathrm{B})]=\lambda_{1} \lambda_{2}+\lambda_{1} \lambda_{2}+\lambda_{1} \lambda_{2}$

Equation 2-21

$\mathrm{I}_{3}=\mathrm{J}_{2}=\lambda_{1}^{2} \lambda_{2}^{2} \lambda_{3}^{2}$

Equation 2-22

where $\lambda_{\mathrm{i}}(\mathrm{i}=1,2,3)$ are the principal stretches of $\mathrm{F}$. As the material is incompressible $\mathrm{I}_{3}=1$.

$W=C_{10}\left(I_{1}-3\right)+C_{01}\left(I_{2}-3\right)+C_{11}\left(I_{1}-3\right) \cdot\left(I_{2}-3\right)+C_{20}\left(I_{1}-3\right)^{2}+C_{30}\left(I_{1}-3\right)^{3}$

Equation 2-23

where $\mathrm{C}_{\mathrm{ij}}$ are the constants, which are determined by fitting the equation to the experimental results.

A hyperelastic constitutive model was generated to describe human coronary artery and plaque for this study.

\subsection{Human coronary arteries}

The aorta is the largest artery in the human body. It extends from the left ventricle of the heart and carries oxygen-rich blood for distribution throughout the body via an intricate system of smaller, branch arteries. The right and left coronary arteries stem from the bulges at the origin of the ascending aorta, immediately distal to the aortic valve (Figure 2-6), and supply blood for the heart muscles or myocardia. Severe narrowing and blockage (stenosis) of these arteries can cause angina and myocardial infarction or heart attack. Stents are used to prevent such blockages and keep the arteries open for blood flow.

Drug eluting stent designs such as polymeric helical devices are mainly used to prevent instent restenosis. Although the risk of in-stent restenosis exists in every artery, its likelihood is greater for some arteries over others. The Left Anterior Descending (LAD) artery, for example, is the anterior interventricular branch of the left coronary artery and is known to carry a higher risk of in-stent restenosis than most other arterial segments [Kastrati, 1997; Hausleiter, 2002]. Therefore, the present study focused on the LAD artery to evaluate stenting behaviour within an area prone to injury and subsequent re-narrowing of the arterial passageway.

Generally, stenting procedures are performed on arteries diagnosed to be beyond the "significant condition," which means they have narrowed beyond an allowable percentage of the normal arterial diameter. For main arteries such as the LAD artery, the significant condition is defined as $50 \%$ stenosis, whereas for the other less significant arteries of the heart, the limit is 
70\% [ACCF/AHA/SCAI Guideline, 2011]. A 50\% stenosis means the diameter of the affected portion of the artery is $50 \%$ less than its normal diameter.

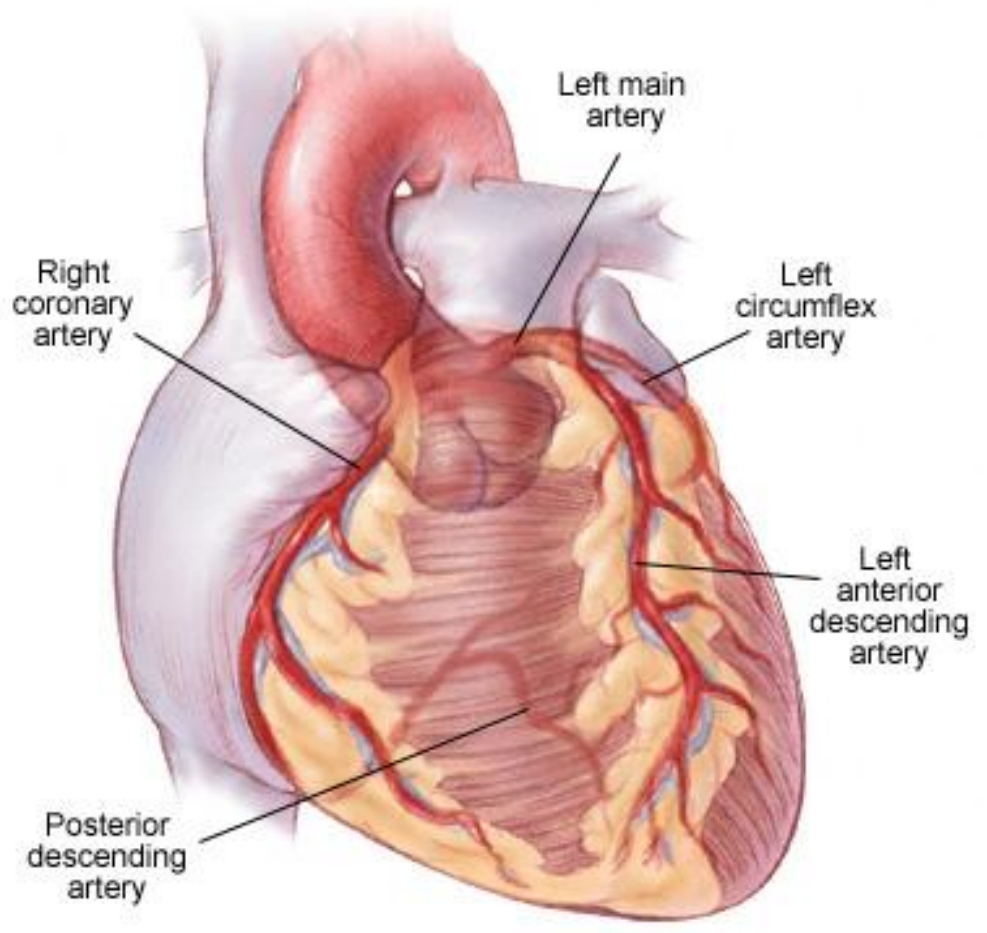

(9) medmovie.corn

Figure 2-6 Schematic representation of the Ascending Aorta and its branching arteries [bostonscientific.com].

To understand the mechanical properties of a human artery, it is necessary to know its basic structure. Human artery walls have three layers: the inner layer or Intima (Tunica Intima), the middle layer or Media (Tunica Media) and the outer layer or Adventitia (Tunica Adventitia) (Figure 2-7). The complex structure of arterial walls allows activation of different layers under different loading conditions. From a mechanical point of view, the selective activation of different layers of artery wall in different loading conditions is very important. 


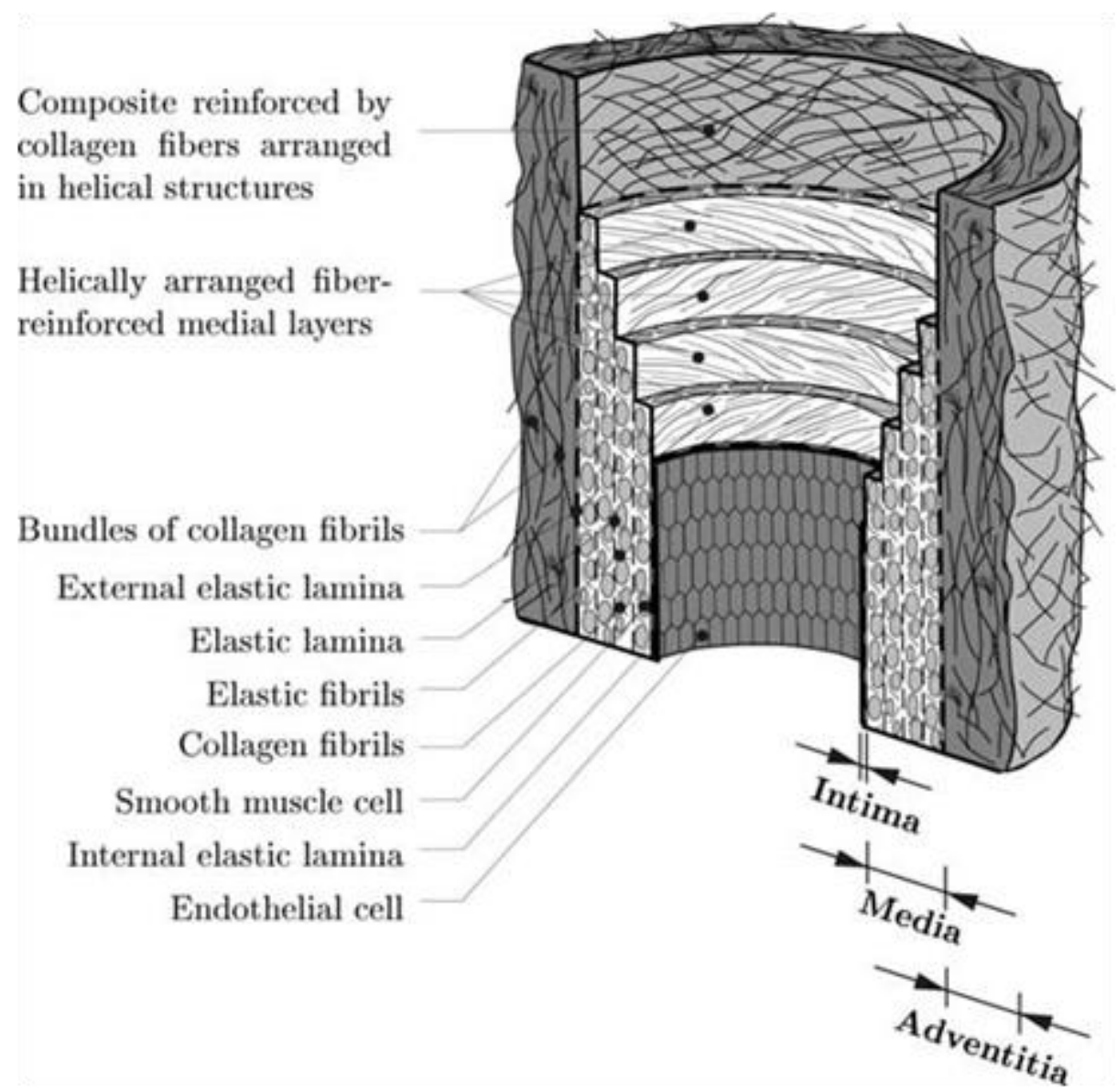

Figure 2-7 Tissue structure of an artery [Holzapfel, 2005].

The intima, the innermost layer of the artery wall, is made up of an elastic membrane lining and smooth endothelial cells and is in direct contact with the blood stream. In human coronary arteries, this layer is thicker than the other two layers and contributes significantly to the mechanical behaviour of these blood vessels (in the other, non-coronary human arteries, the intima is thinner, as shown in Figure 2-7). Therefore, the intima must be a key consideration in the mechanical analysis of human coronary arteries [Holzapfel, 2005]. The media, or middle layer of the artery wall, is made up of smooth cells and elastic tissue. The media can be distinguished from the intima and adventitia by its fibrous layers. The number of fibrous layers in the media varies from 1 (small arteries) up to 4 or 5 layers (larger arteries). Among the three artery wall layers, the media contributes the most to the mechanical properties of the blood vessel, particularly to the physiological range of pressure each vessel normally operates under or can accept. In some mechanical analyses, the artery is modeled as a single media layer [Pant, 
2012]. However, due to the mechanical contributions of the intima and adventitia, especially in the high pressure range, a single-layer approach is not an appropriate strategy for accurately modeling human coronary arteries. The adventitia is the outermost layer of any tubular organ, including the blood vessels, and is made up of elastic (such as elastin) and collagenous fibers. Considered a connective tissue, the adventitia has a significant mechanical contribution when pressure within the artery is above its physiological range.

From a mechanical point of view, an arterial wall is assumed to be incompressible [Carew, 1968; Dobrin, 1969]. The Cauchy stress-stretch behaviour of the arterial wall always has a J-like shape (Figure 2-8) in which the elbow of the curve $\left(\lambda_{e}, \sigma_{e}\right)$ separates arterial compliance, which occurs at relatively low pressures, with increased arterial stiffness under deformation, which occurs at high blood pressures. In a healthy artery, the physiological loading range is below the transitional elbow section.

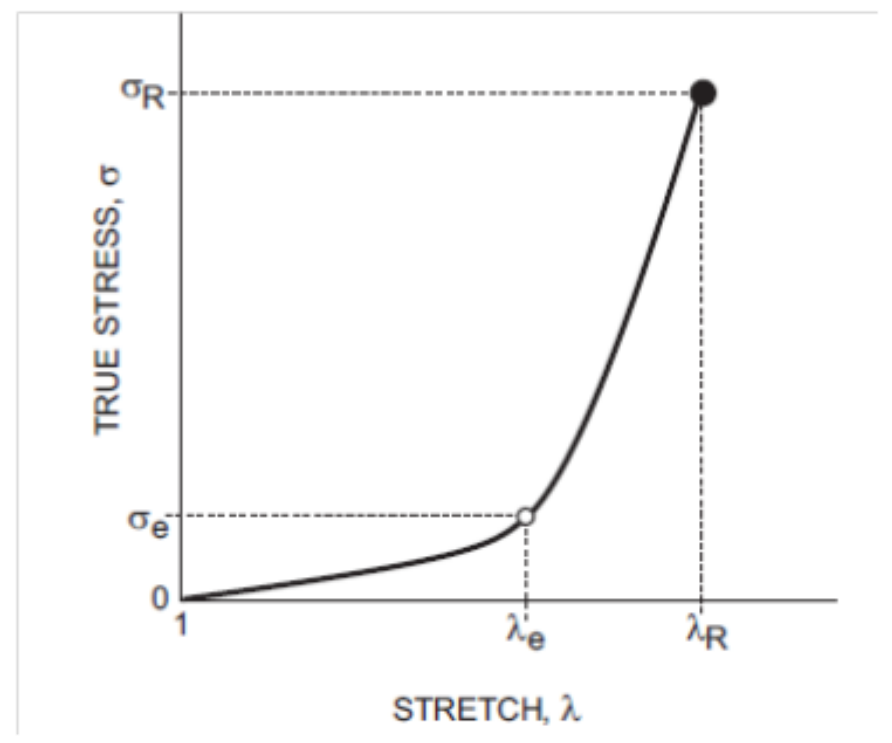

Figure 2-8 Typical true stress-stretch curve of an artery [from Claes, 2010].

In the low stretch domain (less than $30 \mathrm{kPa}$ ), the part of the artery wall with non-collagenous matrix material, namely the intima and to a lesser extent the media, is active and bears the load [Holzapfel, 2005]. The compliant part of the curve is an elastin-dominated zone where the load is carried mainly by the soft tissue. As the artery's inner pressure increases, the helically braided collagen in both the media and adventitia layers reorient into the circumferential direction to sustain the load. The gradual fiber engagement phase can be seen as the elbow zone of the curve. 
The stiffer part of the curve is the collagen-dominated zone [Claes, 2010], where almost half of the load is carried by the collagenous matrix of the artery wall. However, even in this area more than half of the load is still sustained by the intima [Holzapfel, 2005]. Near the maximum stretch level of the artery, all collagenous fibers in the adventitia are activated and the adventitia transforms into a stiff "jacket-like" layer to protect the artery from overstretching and rupture [Schulze-Bauer, 2002].

In a healthy artery the physiological inner artery pressure range sits squarely in the compliant (elastin-dominated) zone. However, with age the arterial wall stiffens, shifting the elbow position of the curve and affecting the stretching capacity of the arterial wall during a normal physiological cycle [Claes, 2010]. The elbow stress $\left(\sigma_{\mathrm{e}}\right)$ and the compliance of the arterial wall decrease with age [Claes, 2010; Ozolanta, 1998]. In a healthy aged coronary artery the elbow stress decreases to $50 \mathrm{kPa}$ [Claes, 2010].

The rupture point in the artery stretch-stress data usually falls on the rupture point of the softest layer of the artery wall, i.e., the Intima [Holzapfel, 2005]. The rupture stress also decreases with age and can drop from $1500 \mathrm{KPa}$ in a healthy young coronary artery to $39 \mathrm{KPa}$ in a healthy aged coronary artery [Claes, 2010; Holzapfel, 2005]. Generally, the artery wall starts weakening when people are between 30 to 40 years of age [Claes, 2010, Vande Geest, 2004].

\subsubsection{Acquiring mechanical properties of arteries}

Designing new therapeutic devices to treat coronary artery disease and other cardiac issues requires detailed knowledge of the mechanical properties of human arteries in conditions as similar as possible to their actual working conditions inside the human body. Very few studies have examined the mechanical properties of human arteries [van Andel, 2003, Holzapfel, 2005, Claes, 2010, Karimi, 2013, Kural, 2012; Yang, 2009]. The lack of reliable mechanical property data [Claes, 2010] hampers the application of numerical methods in predicting arterial responses to medical interventions.

Most available data on arterial tissue mechanics have been collected from animal samples [Bund, 1996; Gow, 1974; Kang, 1995; Lu, 2003; Lu, 2004; Patel, 1970; Vito, 1982]. Despite some similarities, there are fundamental differences between the coronary arteries of animals and humans. Unlike animal arteries, human coronary arteries have a considerably thick Intimal layer that contributes to the mechanical properties of the vessel [Holzapfel, 2005]. Furthermore, 
the Intimal layer grows and thickens as the individual grows [Stray, 1992] and becomes the dominant layer of an old coronary artery. Therefore, there is a huge difference between the stretching capacities of human and animal arteries. For instance, porcine arteries are far more flexible and stretchable than human coronary arteries. Porcine arteries can stretch up to $60-70 \%$, while human arteries can stretch up to $20 \%$ [van Andel, 2003]. Therefore, while the stretchstress data obtained from animal studies can provide us with some insight into the changes that occur during an intervention, they are not suitable for mechanical studies of stent deployment. Furthermore, since the structural and mechanical properties of an artery depend on its position in the arterial tree and also on the individual's medical history [Canham, 1989; Hirai, 1989; van Andel, 2003], the mechanical properties reported for other arteries in the human body [Lally, 2005; Loree, 1994] may not be generalizable to coronary arteries.

Ideally, the mechanical properties of a tissue should be measured in vivo to account for all biological factors. The limited studies performed on human samples can be divided into in vivo and in vitro studies. Due to the inherent accessibility and ethical limitations placed on studies involving living human organs, in vivo measurements are limited to arterial responses in conditions only up to two or three times normal physiological pressure [Ozolanta, 1998; Williams, 1999; Tajaddini, 2005]. Therefore, the behaviour of the tissue in these studies is not representative of the behaviour that occurs under interventional loading conditions (i.e., during angioplasty and stenting) [Claes, 2010]. in vivo studies have primarily examined the module of elasticity of arteries in the physiological pressure range, i.e., 0-240 $\mathrm{mmHg}$ (or 0-31.9974 $\mathrm{kPa}$ ), while the normotensive systolic blood pressure is $100-120 \mathrm{mmHg}$ (or $13.3322-15.9987 \mathrm{kPa}$ ).

Recently, however, a limited number of in vitro experimental studies have examined the mechanical properties of coronary arteries operating beyond their physiological pressure range [van Andel, 2003] all the way up to the "rupture point" or "failure point" [Holzapfel, 2005; Claes, 2010; Karimi, 2012; Kural, 2012; Yang, 2009]. The rupture point is defined as the first point at which one of the layers of the artery wall fails and can be identified by the first sudden drop in the stress-strain curve [Claes, 2010].

One way of analyzing arterial tissue mechanics is to measure the properties of each layer of the artery wall separately. This method is called "layer specific testing." Holzapfel and colleagues are pioneers in layer specific testing [Holzapfel, 2005]. They conducted tensile tests up to the fracture point on samples obtained from healthy elderly people. Results were presented 
for each layer of the human coronary artery wall (i.e., Intima, Media, and Adventitia) in two directions (i.e., circumferential and longitudinal) up to the rupture point [Holzapfel, 2005]. Data from the circumferential direction [Holzapfel, 2005] have been widely used in finite element modeling of stent deployment [Zahedmanesh ,2009; Gijsen, 2008; Zahedmanesh, 2010; Gastaldi, 2010; Gervaso, 2008; Grogan, 2011; Boyle, 2010; Pant, 2012]. However, since only healthy arteries were studied, while samples with any sign of atherosclerosis were rejected, the material properties reported by Holzapfel's team are not useful for the present study.

Yang et al. [Yang, 2009] characterized coronary artery samples with slightly thickened Intima layers, but without clear plaque buildup, using uniaxial tests in both the circumferential and axial directions and resulting stress-stretch data were fitted hyperelastic modeling models. Claes et al. [Claes 2010] characterized the mechanical properties, specifically the axial prestretch and stress-strain behaviour, of healthy coronary arteries by exposing whole samples of Right Coronary (RC) and LAD branches to uniaxial in vitro tensile testing up to failure. They reported the true (Cauchy) stress-stretch data from force-displacement results based on the incompressibility assumption. Although Claes and colleagues performed a fairly thorough study of coronary artery tissue mechanics, they did not report the entire stress-stretch data series required to support constitutive modeling of the RC and LAD blood vessels. Kural et al. [Kural, 2012] examined the mechanical properties of human coronary arteries through biaxial testing of thickened Intima tissue, which is the first symptom of plaque buildup. They found that in comparison with healthy artery walls, unhealthy specimens with thick Intimal layers demonstrate higher stiffness $(50-210 \mathrm{KPa})$ and much lower stretching capacity (1-10\%). Similarly, following in vitro uniaxial tensile tests that stressed healthy and atherosclerotic coronary arteries to the rupture point, Karimi et al. [Karimi, 2013] found that at any given true stress level, healthy human arteries demonstrate twice the deformation compared to unhealthy specimens. Some healthy artery samples were stretched up to $200 \%$ without rupturing. Karimi and colleagues [Karimi, 2013] also measured the compressibility of their samples and reported Poisson ratios of 0.497 to 0.498 for unhealthy and healthy arteries, respectively. Although this is a very unique study, it does not provide sufficient information regarding the calculation of the artery's material properties, the experimental procedure for preparing the samples, or sample dimensions. Without the aforementioned specifications, we cannot use the reported stress-stretch data in finite element modeling. 
Table 2-1 provides a summary of published studies on the material properties of human coronary arteries.

Table 2-1 Summary of the published studies on the material properties of human coronary arteries

\begin{tabular}{|l|l|l|l|l|l|}
\hline $\begin{array}{c}\text { First author } \\
\text { and } \\
\text { publication } \\
\text { date }\end{array}$ & Artery & Type of experiment & $\begin{array}{c}\text { Up to } \\
\text { failure } \\
\text { point? }\end{array}$ & $\begin{array}{c}\text { Health } \\
\text { status of the } \\
\text { artery }\end{array}$ & $\begin{array}{c}\text { Layer } \\
\text { specified? }\end{array}$ \\
\hline $\begin{array}{l}\text { van Andel, } \\
2003\end{array}$ & $\begin{array}{l}\text { RC, LAD } \\
\text { and CX }\end{array}$ & Uniaxial & No & Healthy & No \\
\hline Holzapfel, 2005 & RC and & Biaxial & Yes & Healthy & Yes \\
\hline Yang, 2009 & Coronary & $\begin{array}{l}\text { Uniaxial in both } \\
\text { circumferential and } \\
\text { axial directions }\end{array}$ & Not- & $\begin{array}{l}\text { Healthy, with } \\
\text { Intimal }\end{array}$ & No \\
thickening & \\
\hline Claes, 2010 & LAD & Uniaxial & Yes & Healthy & No \\
\hline Kural, 2012 & Coronary & Biaxial & Yniaxial & Yes & Both healthy \\
and & No \\
Karimi, 2012 & LAD & Unhealthy & No \\
\hline
\end{tabular}

$\mathrm{CX}=$ Circumflex coronary artery, LAD = Left anterior descending coronary artery, and RC $=$ Right coronary artery

Hyperelastic models are commonly used to implement artery material properties in finite element code. The present study employed data reported by Yang et al. [Yang, 2009] because it was derived from human coronary artery testing and they provide sufficiently detailed information regarding the experimental procedure. 
While most arteries demonstrate hysteresis behaviour, coronary arteries show very little hysteresis behaviour due to their muscular nature [Holzapfel, 2005]. Therefore, in the present study we overlook the hysteresis behaviour of artery walls when evaluating the arterial response to stent deployment.

\subsubsection{Plaque material properties}

There are two methods for measuring the mechanical properties of plaque. The first method is based on classic mechanical testing of dumbbell- or rectangular-shaped samples. Samples are tested under various loading conditions until they rupture, i.e., experimental testing continues up to the failure point [Lee, 1991; Loree, 1994; Holzapfel, 2004; Karimi, 2013]. Histological studies are also usually performed; however, histological testing can only be performed in vitro and is not directly useful for patient-specific testing where the properties of a live tissue (i.e., artery) are targeted.

The second method for measuring the mechanical properties of arterial plaque provides a work-around for the complex and irregular geometries typical of these fatty deposits. Specifically, this method applies non-invasive, image-based techniques such as Magnetic Resonance Imaging (MRI) and Intravascular Ultrasound (IVUS) to capture the mechanical and geometrical properties of plaque in vivo [Yang, 2009; Karimi, 2008]. This method can capture images of the artery under physiological loading and accurately measure subsequent strain. Armed with data that describes the strain level of loaded and unloaded samples, the artery's specific loading range, its composition and mechanical properties, researchers can measure the plaque stress level. The data collected in this step is used to obtain patient-specific image-based measurements.

The loading range produced by a typical angioplasty and stenting procedure is beyond the physiological range of pressure and is in fact closer to the rupture pressure. Therefore, in the present study plaque mechanical data measured using traditional mechanical testing was used to support the modeling activity.

Typically, coronary artery plaques are composed of cellular, calcified, and hypocellular tissues [Kragel, 1989; Lee, 2000; Loree, 1994; Karimi, 2013]. Although each plaque contains a combination of these three tissue types, one type may be dominant in each plaque's composition. 
For example, plaques dominated by Cellular tissue are the softest type. There is no known correlation between the position of the artery and plaque composition [Kragel, 1989].

Plaque rupture is life threatening and plaque vulnerability to rupture has been studied extensively [Falk, 1995]. Plaque vulnerability is directly related to its composition rather than its volume [Falk, 1995]. Soft lipid rich plaques with thin caps are more dangerous than calcified hard plaques and are more prone to rupture even under physiological pressure loadings [Fernandez-Ortiz, 1994]. Loree et al. [Loree, 1994] measured the stress-strain curves of three plaque types taken from human coronary artery samples, and reported engineering stress-strain data for each composition and identified that cellular plaques are the softest and more vulnerable to rupture. Since softer plaques are more vulnerable to rupture, the present study focuses on the softest plaques, i.e., the Cellular plaques. 


\section{Chapter 3 \\ Material characterization (PLLA and PLGA)}

Generally, a primary step in finite element (FE) model development is implementation of structural material models into the code. Characterization of individual material responses is essential to creating robust FE models. If reliable data is not available in the literature to properly characterize physical material properties, direct mechanical testing should be undertaken.

Polymeric materials usually exhibit time dependent (viscous) and process (load history) dependent physical properties, both of which can be altered by environmental conditions (e.g., temperature and immersion in water). Therefore, care should be taken in using data available in the literature.

Since PLLA and PLGA properties have not been investigated extensively in in vitro conditions to determine the effects of temperature, immersion in water and soaking time, mechanical testing of these materials was undertaken using thin film samples fabricated by solution casting [Frank 2004]. The PLLA and PLGA (53/47) films used for this study had a molecular weight of $1.96 \times 10^{6}$ and $3.2 \times 10^{4}(\mathrm{~g} / \mathrm{mol})$, respectively. PLGA is an amorphous material and the particular material used in this study had a glass transition temperature of $37.5^{\circ} \mathrm{C}$, while PLLA, which is typically about $30 \%$ crystalline, had a glass transition temperature of $54.8^{\circ} \mathrm{C}$.

Generally, polymers have lower stiffness than other structural materials, such as metals. Therefore, the devices used to measure the mechanical properties of polymers must be more precise, capable of measuring small quantities and have smaller maximum force limits. These devices can be either force controlled or displacement controlled. The present study employed a force-controlled instrument, specifically a dynamic mechanical analyzer (DMA), to measure the mechanical properties of PLLA and PLGA samples.

\subsection{Dynamic mechanical analyzer (DMA) specifications}

A Dynamic Mechanical Analyzer (DMA) is a thermal analytical instrument used to measure both intrinsic and extrinsic mechanical properties of a given material. The DMA is a force controlled device; it measures the force required to produce a small deformation in the sample. 
These instruments employ different clamps to test specific sample types (e.g., fiber, films, and gels).

In this study, a TA Instruments 2980 Dynamic Mechanical Analyzer was used to characterize the mechanical and viscoelastic properties of PLLA and PLGA thin films, employing two different clamps - a tension-film clamp (Figure 3-1) and a film submersion clamp (Figure 3-2). Mechanical loading was controlled using Instrument Control Software: Thermal Advantage 99.

While the load range of the DMA instrument is relatively small, at $18 \mathrm{~N}$, it is adequate for the soft polymer materials studied in this research.

The DMA was used to perform two types of mechanical testing: tensile and creep tests.

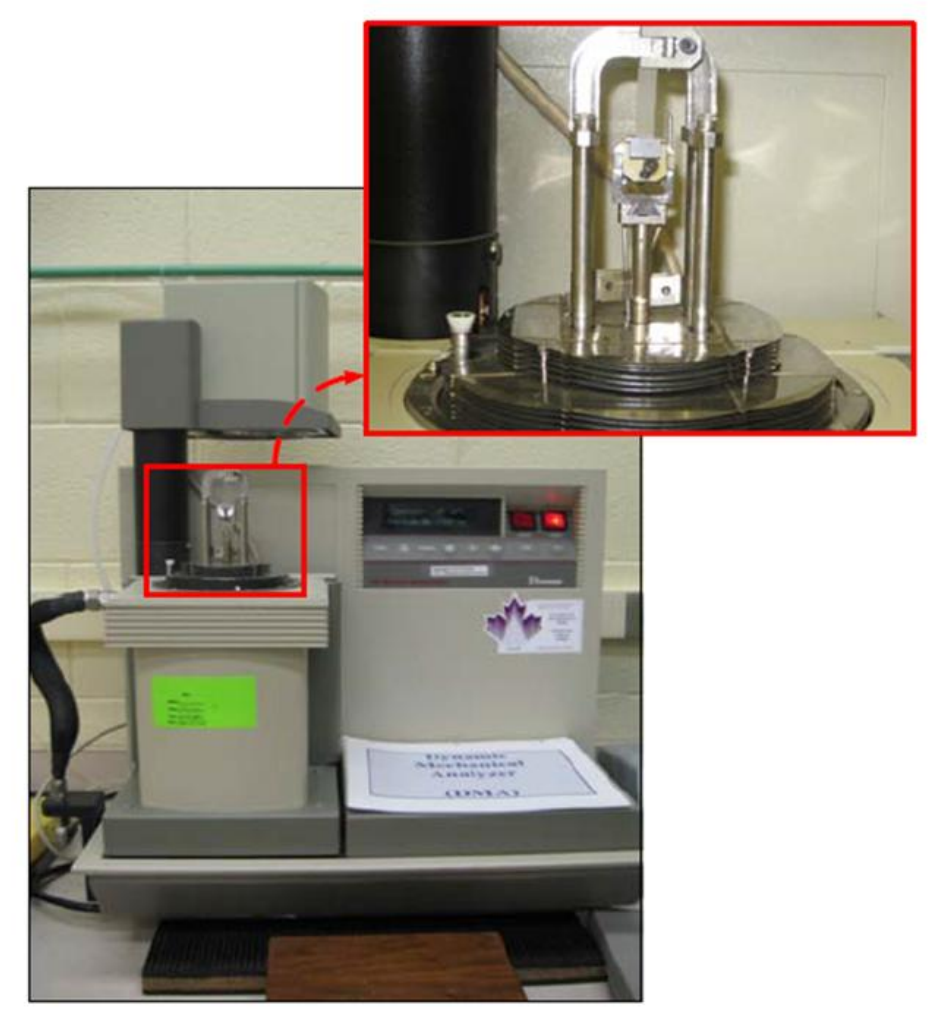

Figure 3-1 Film tension clamp (DMA 2986). 


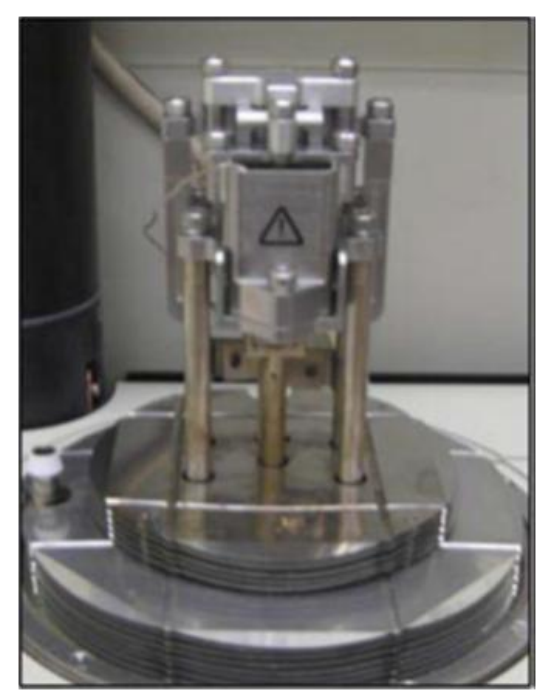

\section{Figure 3-2 Immersed film tension clamp (DMA).}

\subsection{Tensile tests}

Since experimental stent expansion tests conducted by the collaborator researcher at Nanyang technological University (NTU) revealed no evidence of delamination or a transition region between materials in the stent, the mechanical properties of PLLA and PLGA were studied separately. For each test condition, five samples of PLLA and four samples of PLGA were cut from fabricated films to a nominal length of $30 \mathrm{~mm}$ using a guillotine cutter. Different sample widths were used ( $4 \mathrm{~mm}$ for PLLA and $5 \mathrm{~mm}$ for PLGA) to maximize the width while remaining below the maximum allowable force for the test apparatus $(18 \mathrm{~N})$. Five samples were tested for each condition.

PLLA and PLGA samples were tested under the following four conditions:

i. Room temperature in air (reference)

ii. $\quad 37^{\circ} \mathrm{C}$ in air

iii. Room temperature in water

iv. $\quad 37^{\circ} \mathrm{C}$ in water

Condition (iv) most closely approximates actual stenting or in vitro conditions. The first 3 sets of tests were conducted to provide a comparison against in vitro results and to investigate the effect of immersion in water and temperature on the mechanical properties of the PLLA and PLGA samples. In-air tests were conducted with dry samples at room temperature $\left(\sim 25^{\circ} \mathrm{C}\right)$ and $37^{\circ} \mathrm{C}$, while in-water tests were performed in an immersion tank with a $\mathrm{pH}$ of 7.4, conditions similar to 
human blood. The in-water tests were conducted after soaking the samples in a $37^{\circ} \mathrm{C}$-water tank for 30 minutes [communication with NTU], a procedure often used to soften the materials before stenting.

Table 3-1 Summary of tests conducted to examine the effect of temperature, immersion in water, and pre-conditioning on the mechanical properties of PLLA and PLGA.

\begin{tabular}{|l|l|l|l|l|l|}
\hline Type & Soaking (a) & Exposure condition (b) & Temp. (a) & Temp. (b) & Clamp \\
\hline i & NO & Air & Room temp. & Room temp. & Tension-film \\
\hline ii & NO & Air & $37^{\circ} \mathrm{C}$ & $37^{\circ} \mathrm{C}$ & Tension-film \\
\hline iii & 30 min in water & Water & Room temp. & Room temp. & Submersion \\
\hline iv & 30 min in water & Water & $37^{\circ} \mathrm{C}$ & $37^{\circ} \mathrm{C}$ & submersion \\
\hline
\end{tabular}

Generally, stents made of PLLA exhibit limited expansion rates to ensure sufficient ductility during the expansion process. Grabow et al. [Grabow, 2005] demonstrated that changing the loading rate from $0.1 \mathrm{~N} / \mathrm{min}$ to $1 \mathrm{~N} / \mathrm{min}$ and then $10 \mathrm{~N} / \mathrm{min}$ produces an increase in the modulus and yield limit while reducing the elongation to failure, typical of polymeric materials. To achieve the required elongation for stent expansion, the loading rate should be limited to $1 \mathrm{~N} / \mathrm{min}$ which would cause the stent to expand within 3-7 $\mathrm{min}$. This is equivalent to expanding a wire mesh stent with a loading rate of 1 bar/min [Bünger, 2007]. Therefore, DMA testing was conducted with a loading rate of $1 \mathrm{~N} / \mathrm{min}$.

Tension test results (engineering stress and strain) show that the material ductility of both PLLA (Figure 3-3) and PLGA (Figure 3-4) increased with soaking time and increasing temperature. Error bars show the upper and lower limits observed during the experiment. The ' $\mathrm{x}$ ' in the figures indicates failure of the sample, while the arrow indicates that the sample did not fail during the test. In general, the PLLA samples exhibited necking prior to failure, while the PLGA samples did not show this localization when tested in water. Of particular significance, both materials reached the deformation limit of the test apparatus (approximately 50\% elongation) without failing when tested in water, demonstrating a significant increase in material 
elongation. Increasing temperature reduced the stiffness and yield strength of both materials, as expected.

The elongation of PLLA, the structural material in the stent, exceeded $40 \%$, indicating that this material was suitable to accommodate the required plastic deformation on expansion to achieve fixation in the artery (discussed in Chapter 4). Therefore, despite its brittle nature in dry conditions, PLLA is a suitable stent material for in vitro applications. Immersion in water and temperature were found to be important factors (Figure 3-3 and 3-4) in increasing the ductility and decreasing the yield stress of both materials.

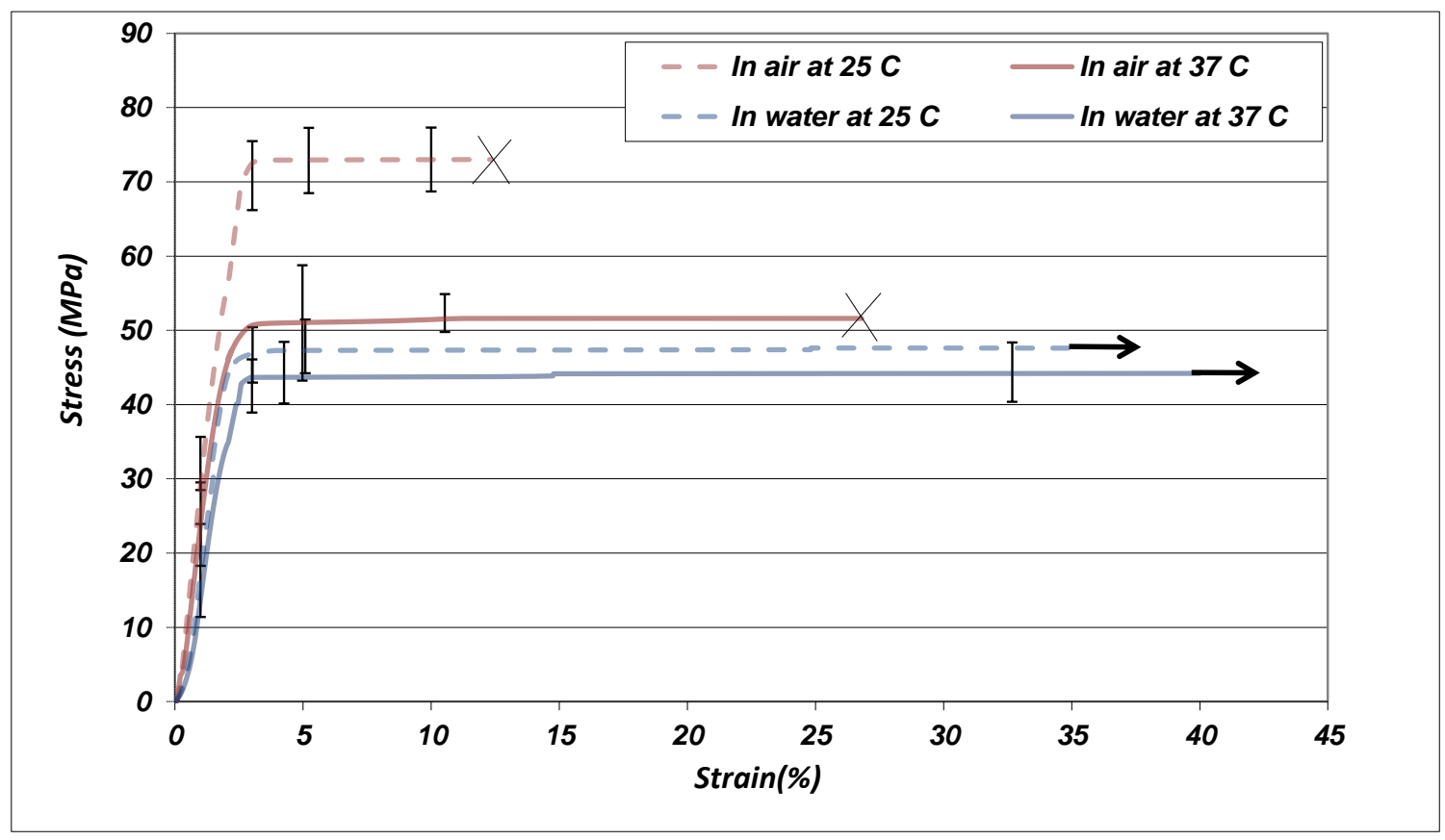

Figure 3-3 Stress-strain results for PLLA samples tested at $25^{\circ} \mathrm{C}$ and $37^{\circ} \mathrm{C}$ in air and water-bath using a DMA tension clamp. Error bars show the upper and lower limits observed during the experiment. The ' $x$ ' in the figures indicates failure of the sample, while the arrow indicates that the sample did not fail during the test. 


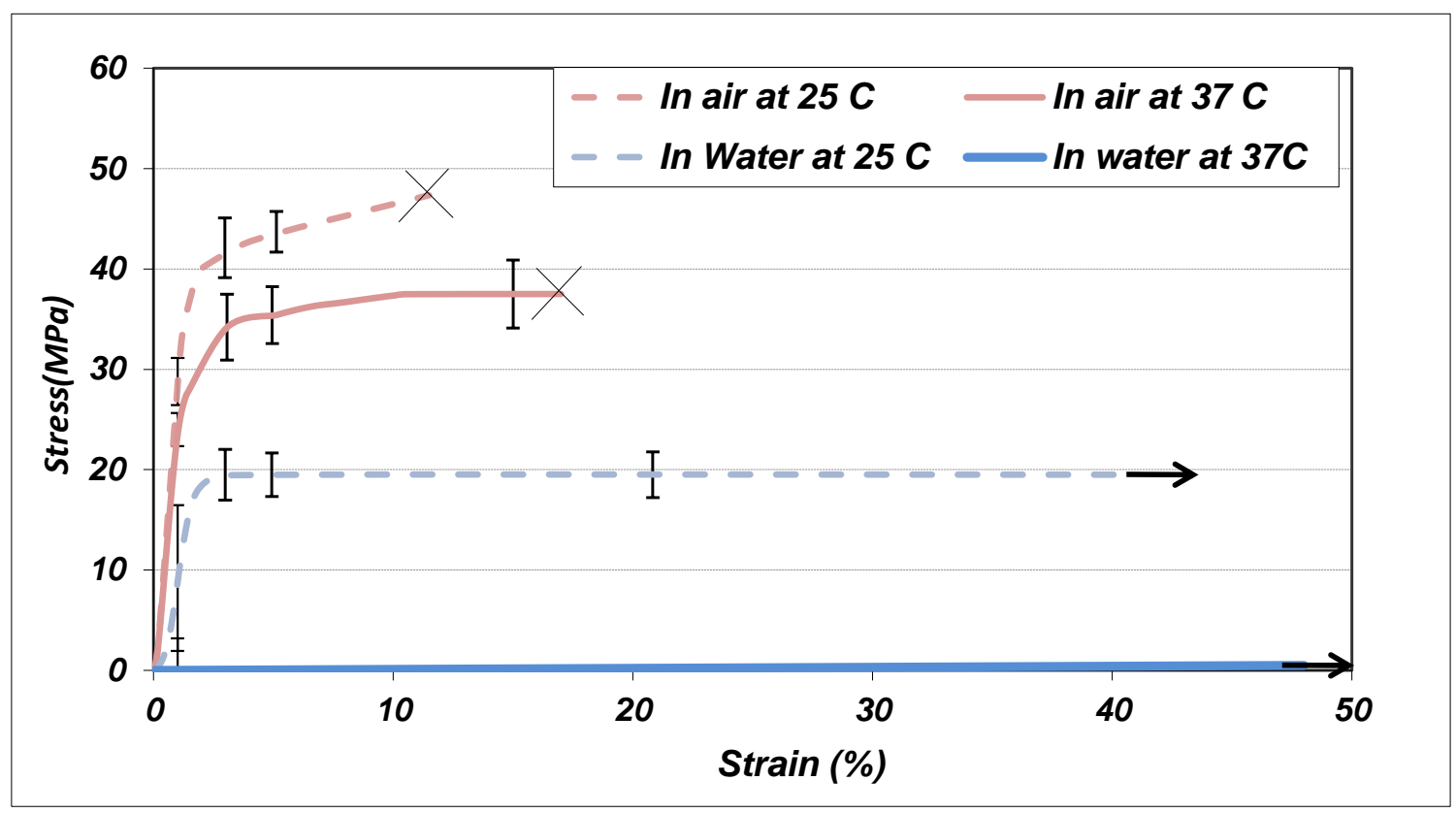

Figure 3-4 Stress-strain results of PLGA samples tested in $25^{\circ} \mathrm{C}$ and $37^{\circ} \mathrm{C}$ in air and in water-bath using a DMA tension clamp. Error bars show the upper and lower limits observed during the experiment. The ' $x$ ' in the figures indicates failure of the sample, while the arrow indicates that the sample did not fail during the test.

As shown in Figure 3-4, PLGA samples reach the maximum measurable strain. The sample reaches the maximum measurable strain after applying a tensile stress of $0.2 \mathrm{~Pa}$. Both increasing temperature and immersion in water increases material ductility. However, the combination of elevated temperature and immersion in water softens the samples, completely depleting their mechanical strength. Uniform expansion model results (described in Chapter 4) show that the elongation limit required to expand the stent to twice its initial diameter is $30 \%$. From the material testing results, PLLA samples can be elongated up to $50 \%$ in in vitro conditions without any sign of breakage (Figure 3-3). 


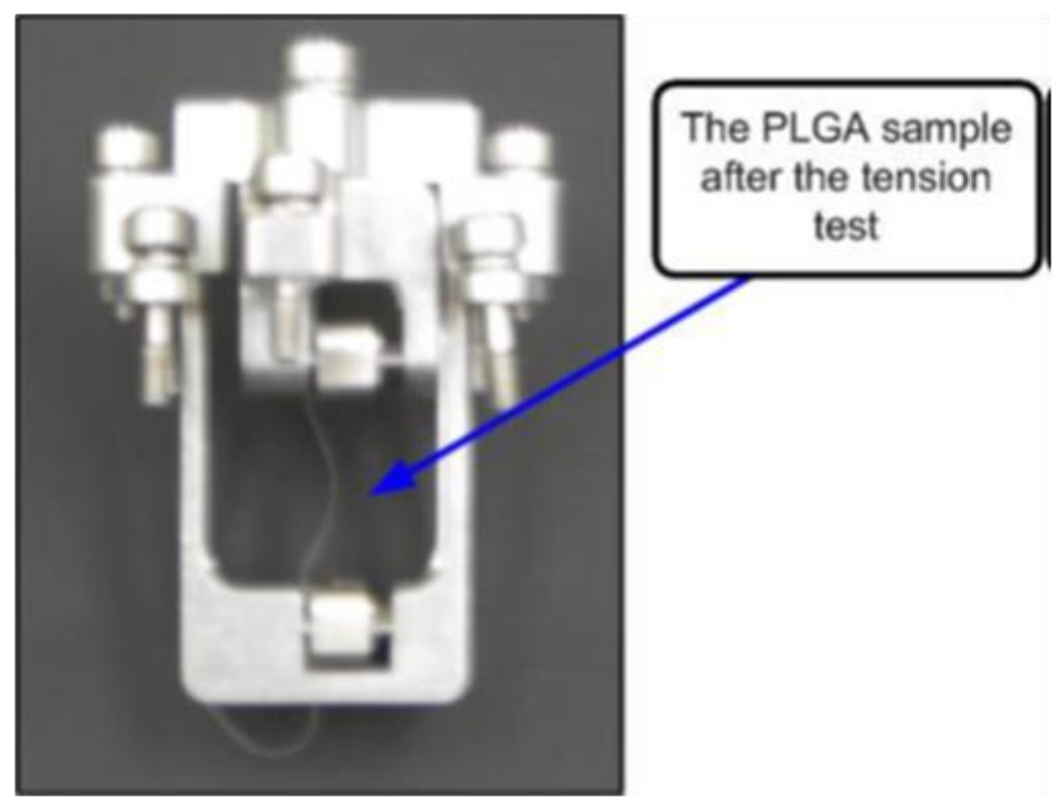

Figure 3-5 Image of a PLGA sample 24 hours after pre-conditioning and tension testing in a $37^{\circ} \mathrm{C}$ water-bath. The sample is gripped in a submersion clamp.

Overall, body temperature and immersion in water similarly affect the mechanical properties of PLLA samples; however, immersion in water demonstrates far greater impacts than temperature, as shown in Table 3-2. In combination, these conditions soften the samples. It was also found that the sample rapidly elongated by more than $50 \%$ and the elongation was permanent, as shown in Figure 3-5. This was expected since the PLGA glass transition temperature is very close to $37^{\circ} \mathrm{C}$. The sample image was captured a day after the experiment; the clamp was removed from the water bath immediately after the test.

Table 3-2 Average properties of PLLA films based on a sample size of five.

\begin{tabular}{|c|c|c|c|c|}
\hline Test condition & i: air- $25^{\circ} \mathrm{C}$ & ii: air-37 ${ }^{\circ} \mathrm{C}$ & iii: water- $25^{\circ} \mathrm{C}$ & $\begin{array}{c}\text { iv: water- } \\
37^{\circ} \mathrm{C}\end{array}$ \\
\hline Tensile strain at yield (\%) & 3.1 & 3.0 & 2.1 & 3.0 \\
\hline Tensile stress at yield (MPa) & 71.1 & 53.2 & 46.2 & 42.3 \\
\hline Young's modulus(GPa) & 2.3 & 1.8 & 2.2 & 1.4 \\
\hline Tensile strain at break (\%) & 12.3 & 26.9 & -- & - \\
\hline Tensile stress at break (MPa) & 73.0 & 59.1 & - & - \\
\hline
\end{tabular}


When exposed to a combination of contact with body fluid and body temperature, PLLA and PLGA films soften and lose their strength; however, these effects are worse for PLGA samples. Since the conditions under testing resemble actual stenting, it can be concluded that the PLGA layer in the bilayer stent design does not contribute to the mechanical strength of the helical stent; it simply acts as a drug-carrying layer. Therefore, the properties of the PLGA layer can be excluded from the FE analysis. Furthermore, future viscoelastic characterization should also focus on PLLA material.

\subsection{Presoaking effects on the stress-strain curve of PLLA samples}

This project is part of wider project initiated at NTU. NTU researchers developed the design and deployment conditions [Venkatraman, 2006A]. In the deployment process, they presoak the stent inside a $37^{\circ} \mathrm{C}$-water tank for 30 minutes before expansion. To evaluate the effect of preconditioning on the stress-strain curve of PLLA samples, a series of tests were conducted. Fresh cut PLLA samples were used for each test. Three series of tension tests were conducted. Samples were tested in the following conditions:

- No soaking and tested in air at room temperature (control)

- Different soaking times in $37^{\circ} \mathrm{C}$ water $(0,10,15,20$, and 30 minutes $)$ and tested in $37^{\circ} \mathrm{C}$ water tank

- 24 hours in air at room temperature and tested in $37^{\circ} \mathrm{C}$ water tank with no pre-soaking 


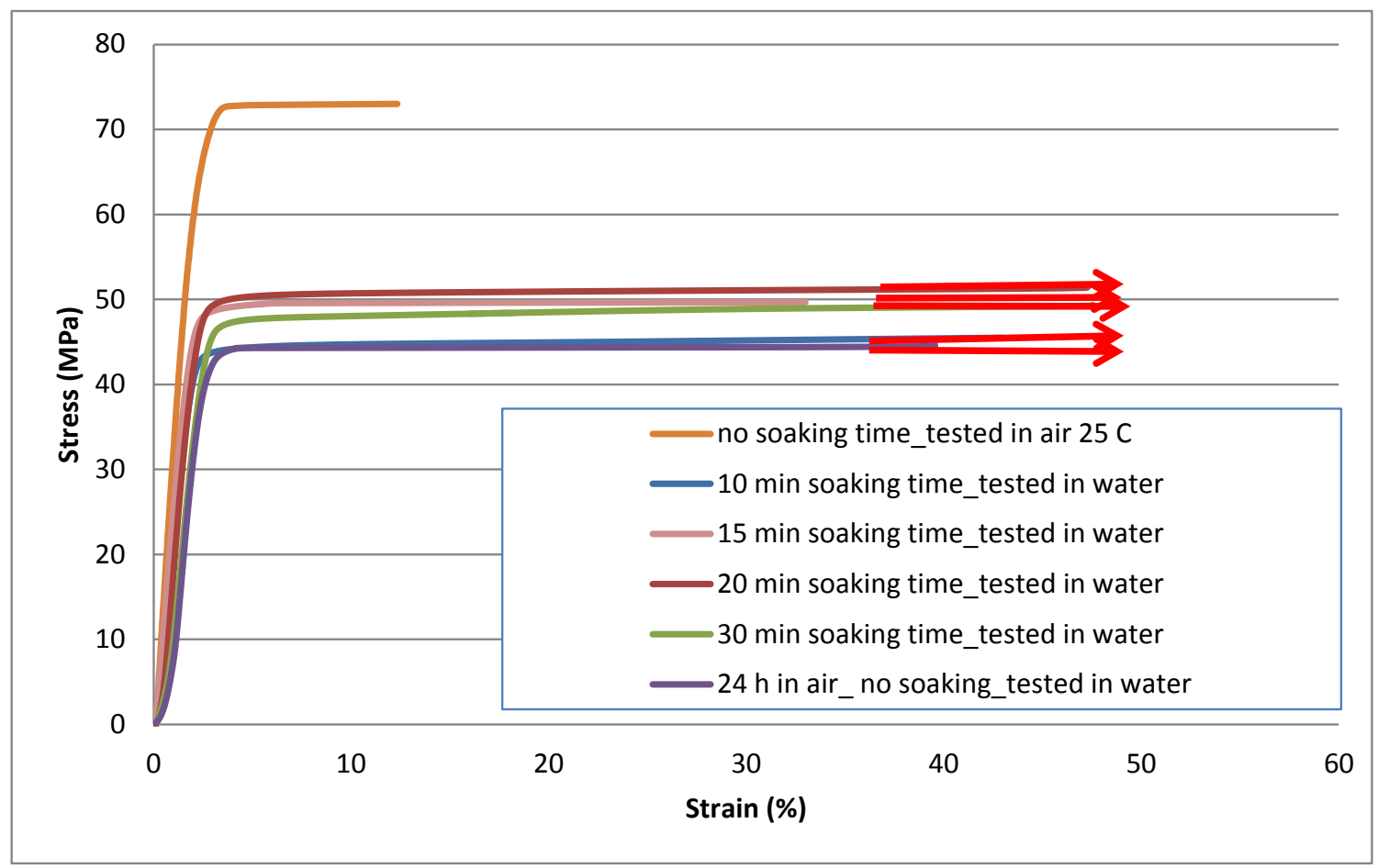

Figure 3-6 Tension tests to evaluate the effect of soaking time on the stress-strain curve of PLLA samples (one sample is tested for each condition).

Results of water tank tests showed that the increased temperature and immersion in water during the deformation is necessary to achieve the required mechanical toughness for deployment (Figure 3-6). The arrows in Figure 3-6 indicate that the sample did not fail during the test, while the cross shows breakage in the sample. Four of the samples were tested after soaking in $37{ }^{\circ} \mathrm{C}$ water and a standard deviation of 5.6 was calculated for the yield point the curve. Temperature and immersion in water requirements reflect in vivo conditions during real-world stent deployment. It appears that the length of pre-soaking time has a minimal effect on the stressstrain relationship. The variability in response is in the range of the standard deviation of the data which is shown in Fig 3-3. Although presoaking time has no effect on stress-strain relationship, the time period of presoaking may be important for the stent's drug delivery performance.

In addition, results of stored-in-air sample testing showed that storing the samples at room temperature degrades their mechanical properties (Figure 3-6). Therefore, material 
samples and, by extension, the stents, should be sealed in a container and stored in a refrigerator kept at a temperature well below their glass transition temperatures.

\subsection{Viscoelasticity tests}

Polymers are viscous materials. The time and temperature dependent properties of the samples were studied using a DMA instrument equipped with an immersed grip.

\subsubsection{PLGA}

For the creep test, PLGA films less than $0.1 \mathrm{~mm}$ thick were fabricated using solution casting. PLGA strip samples were cut from the fabricated films to nominal lengths of $3 \mathrm{~cm}$ and $5 \mathrm{~mm}$ widths using a guillotine cutter.

The creep tests were conducted using a DMA immersed tension clamp. The sample was mounted on the clamp and soaked for 30 minutes in a water tank preheated to $37^{\circ} \mathrm{C}$. A $2 \mathrm{MPa}$ tension load was then abruptly applied to the sample and the deformation was measured. The loading was kept constant for 10 minutes and then removed, at which point the strain recovery of the sample was measured in the immersed condition. One PLGA sample was tested. The sample recovered the strain completely after 56 minutes. In addition, the sample did not neck during elongation, despite very large and abrupt deformation.

As shown from the tension testing results, PLGA samples do not demonstrate structural resistance under tensile loading; therefore, by applying a tensile load, the sample elongates to reach the elongation limit of the test clamp (here, it was 45\%). This is evident from the creep test results shown in Figure 3-7, specifically the straight vertical line at the end of loading section. In addition, similar to the loading profile, the deformation was abrupt and there was no lag between deformation and loading (Figure 3-7). Therefore, PLGA samples do not exhibit time dependent properties during the loading phase and it is acceptable to eliminate the PLGA layer in the FE model.

The relaxation phase of the test results showed time dependent, viscoelastic behaviour (Figure 3-7). The deformation, however, was fully elastic and the sample recovered its original length after the full relaxation period, roughly 30 minutes in this case. This suggests the recoiling behaviour of the stent's PLGA layer is time dependent. However, due to the minimal structural resistance demonstrated by the PLGA layer in the in vitro condition, the PLGA layer in a bi-layer stent will follow the PLLA layer deformation without compromising its mechanical 
properties. Furthermore, this suggests that eliminating the PLGA layer from the entire modeling process (both expansion and uncoiling phase) will not affect the results unless that elimination changes the stent's center of inertia, impacting the bending response of the stent.

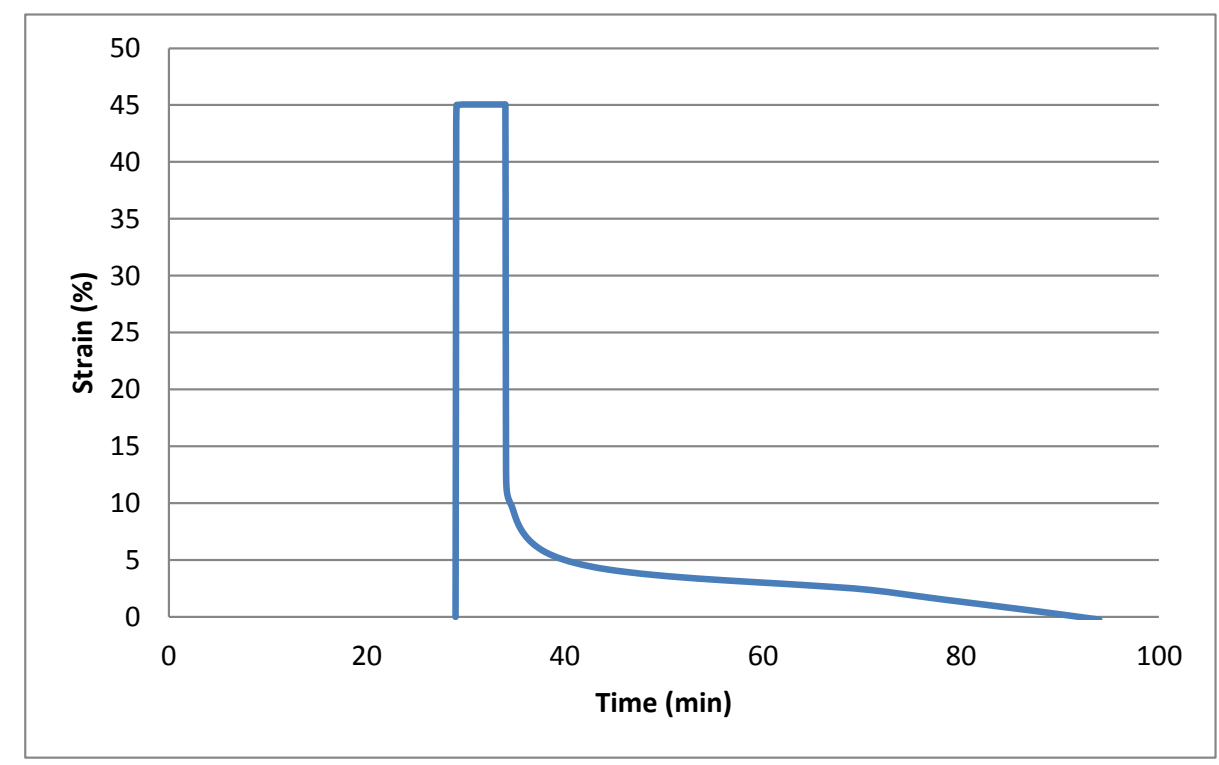

Figure 3-7 Creep test on PLGA sample in $37^{\circ} \mathrm{C}$ water under $2 \mathrm{MPa}$ load after $30 \mathrm{~min}$ soaking in water.

\subsubsection{PLLA}

The viscoelastic behaviour of PLLA samples was measured both in room temperature air and in in vitro conditions $\left(37^{\circ} \mathrm{C}\right.$ water). The strip samples were cut from the fabricated films to nominal lengths of $3 \mathrm{~cm}$ using a guillotine cutter. The width of the PLLA samples was $4 \mathrm{~mm}$.

For the in-air test, a sample was mounted on the tension DMA film clamp. A $25 \mathrm{MPa}$ tension load was abruptly applied to the sample and the strain was measured (Figure 3-8). 


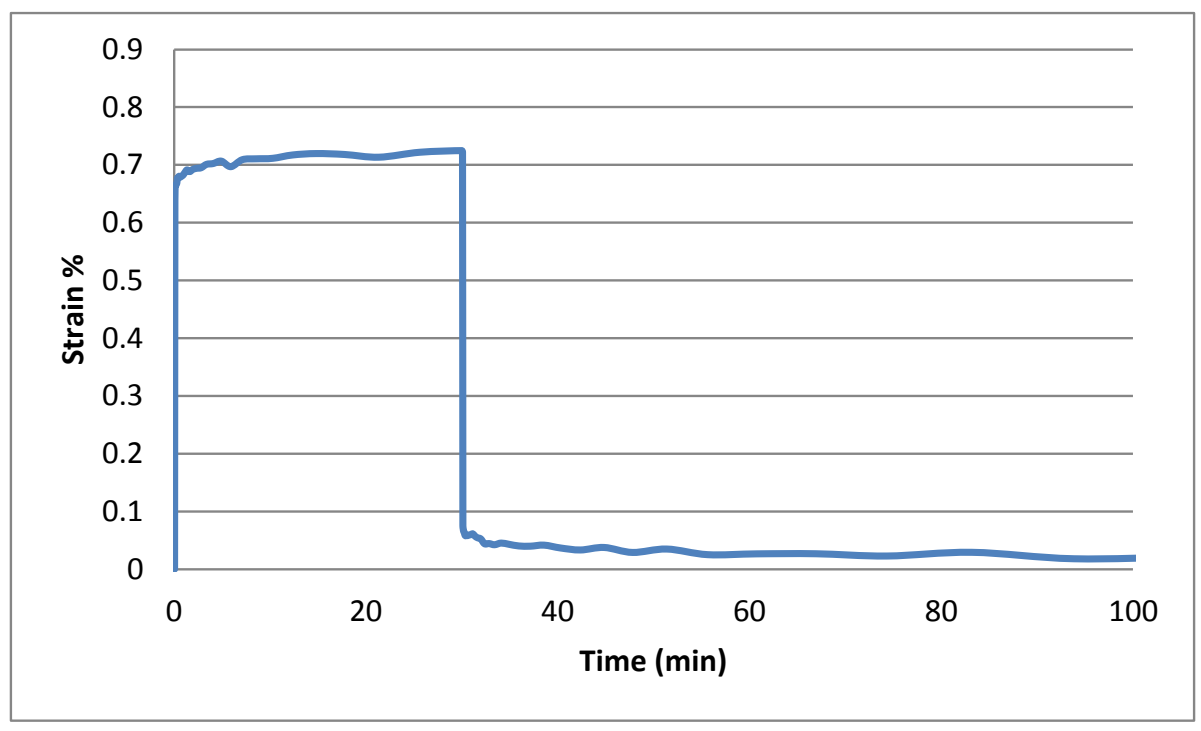

Figure 3-8 PLLA creep test results measured in room temperature air under 25 MPa tensile loading.

The results show that PLLA samples exhibit viscous properties and that there is a lag between the loading and measured strain. The sample reaches equilibrium within the first 5 minutes. After the force was removed, recovery was almost instantaneous. The samples exhibited a small percentage of unrecovered strain (less than $0.05 \%$ ), which decreases over time. However, the sample did not recover completely after 65 minutes.

A series of in-water tests was conducted on PLLA samples, which were mounted to the DMA submersion clamp and then soaked for 30 minutes in a water tank preheated to $37^{\circ} \mathrm{C}$. One sample for each condition was tested. Fresh cut samples were used for each test. Viscoelastic behaviour was measured in the tensile loading range of 10 to $35 \mathrm{MPa}$ (Figure 3-9). A tensile loading range higher than $35 \mathrm{MPa}$ could not be tested due to DMA device limitations. 


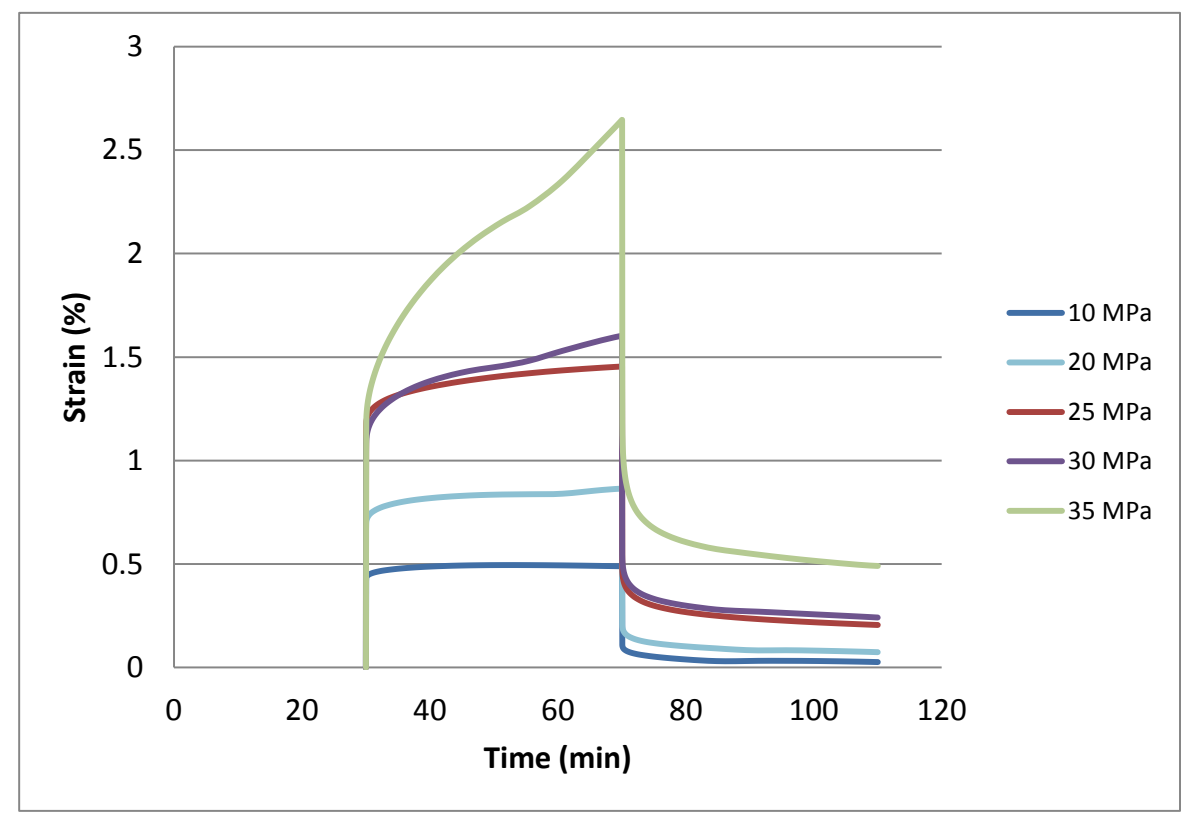

Figure 3-9 In vitro PLLA creep results (in the loading range of 10-35 MPa) following 30 minutes of preconditioning in $37^{\circ} \mathrm{C}$ water .

Under the tensile loading conditions (up to $35 \mathrm{MPa}$ ), the samples are in small strain range (up to $3 \%$ ). The results showed that from very small strain levels, the PLLA samples demonstrate viscoelastic behaviour in vitro. Under 10-20 MPa, the samples gained equilibrium during both creep and recovery in a very short period of time (within 5 minutes). As expected, with increased loading, the samples exhibit nonlinear viscous behaviour and resulting creep and recovery curves are not parallel.

\subsubsection{Evaluating linearity of PLLA samples in vitro (in $37^{\circ} \mathrm{C}$ water)}

In the linear viscoelasticity region, the mechanical strength (i.e., the compliance) of the material is independent of the loading. The creep compliance can be calculated by dividing the strain to the applied stress during creep deformation.

To examine the linearity of the samples, compliance was plotted versus time. Under low levels of stress (10-30 MPa), the difference seen between the curves is caused by the variation in mechanical properties of the polymer samples and is thus acceptable. However, 
under higher levels of stress, $35 \mathrm{MPa}$, the slope of the compliance curve differs considerably from previous curves, indicating nonlinearity.

As in practice, a step force cannot be applied instantly during experimentation. Thus, the nonlinear starting regions of the curves do not represent the effects of the actual loading pattern. In practice, it takes a period of time for the sample to reach the intended force. This causes a level of error in the beginning of the creep curve, as shown in Figure 3-10. In reality, applying stress or strain takes time. However, this portion of load application should not be considered in the material model [Christensen, 1982].

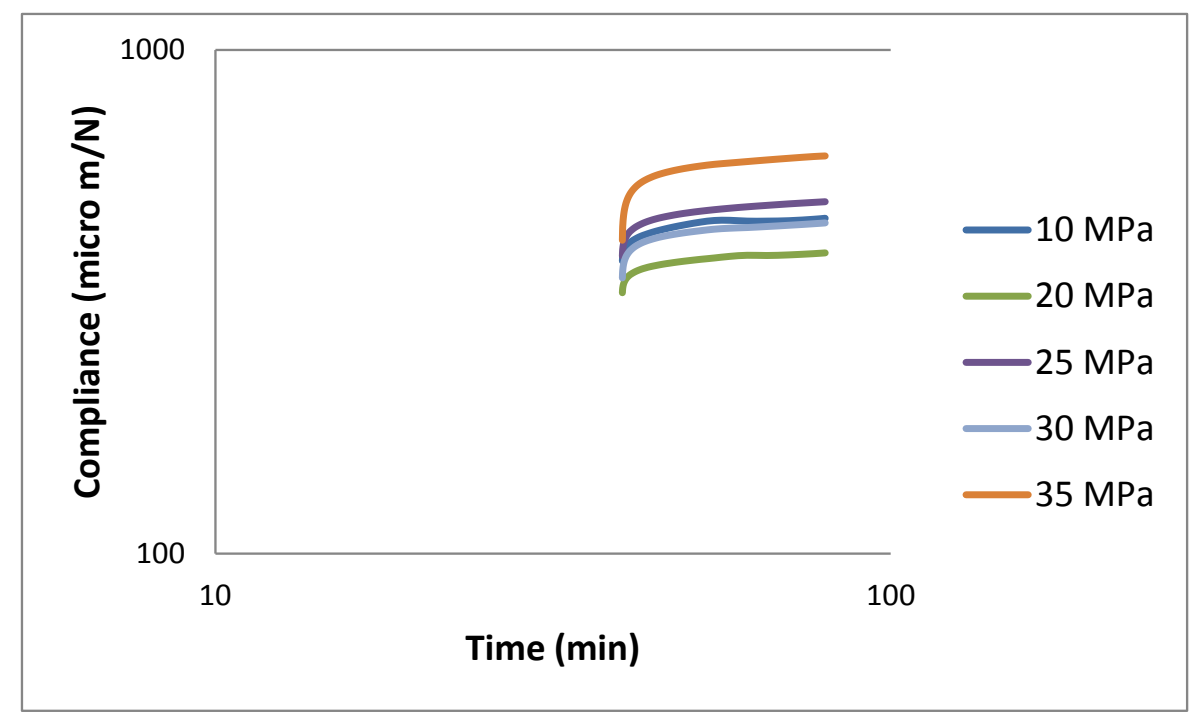

Figure 3-10 Creep Compliance of PLLA samples in vitro.

Creep compliance measurements taken after 10 minutes of applying the load show nonlinearity of PLLA samples in in vitro conditions (Figure 3-11). Changes in creep compliance, however, did not show any clear trend with increasing stress level. 


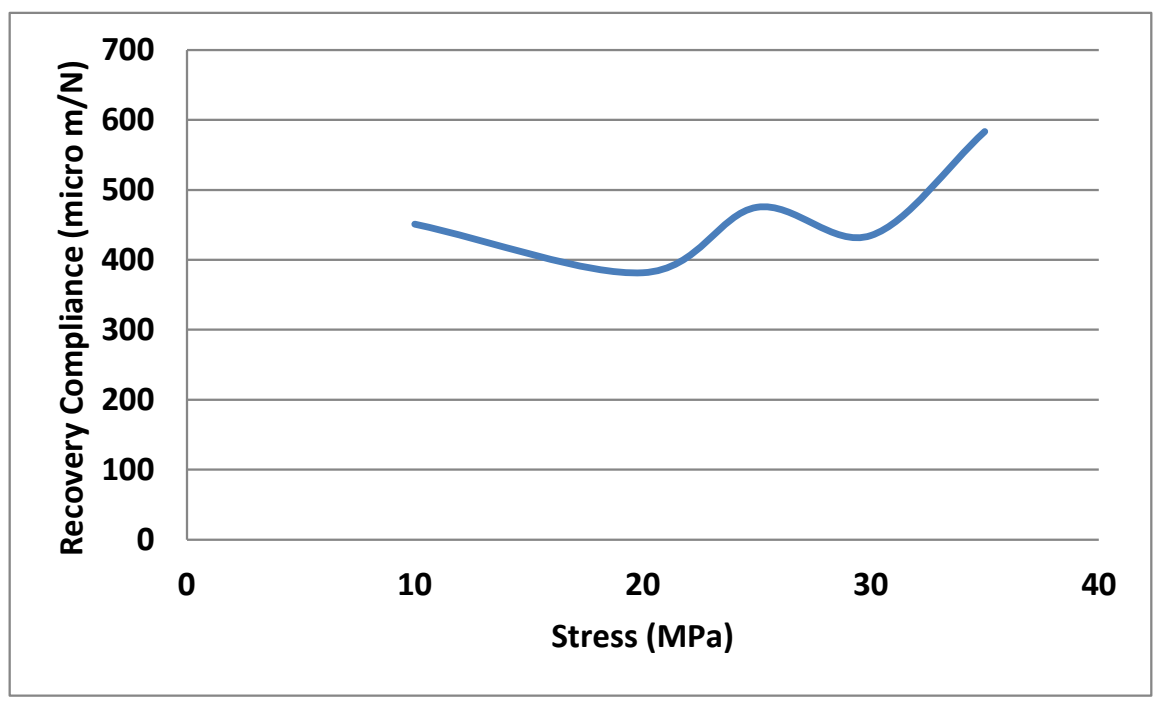

Figure 3-11Creep compliance versus applied stress measured 10 min after applying the force.

\begin{abstract}
Material compliance can also be used to detect nonlinearity during the recovery period. As shown in Figure 3-12, the slope of the recovery compliance curve produced by samples under 35 $\mathrm{MPa}$ creep loads is quite different from those produced by samples under smaller loads. The sample under $30 \mathrm{MPa}$ loading also demonstrated nonlinear behaviour. Therefore, from both creep and recovery compliance curves, it is concluded that the nonlinear viscoelastic behaviour of PLLA samples tested in vitro starts at $30 \mathrm{MPa}$.
\end{abstract}




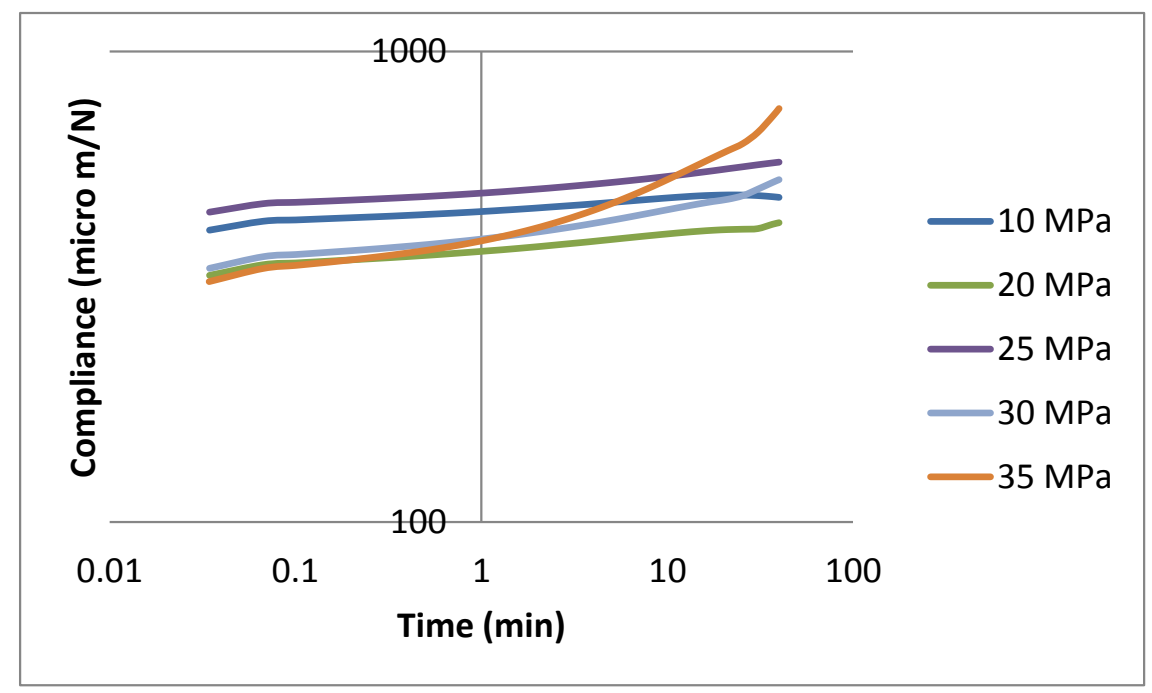

Figure 3-12 Recovery compliance curves of PLLA samples tested in vitro.

Recovery compliance curves built based on measurements taken 10 minutes after removing the load show nonlinearity in PLLA sample behaviour in in vitro conditions (Figure 3-13). Furthermore, changes in recovery compliance did not show any clear trend with increasing stress level. However, in general, the creep and recovery compliance curves follow a similar pattern across stress levels.

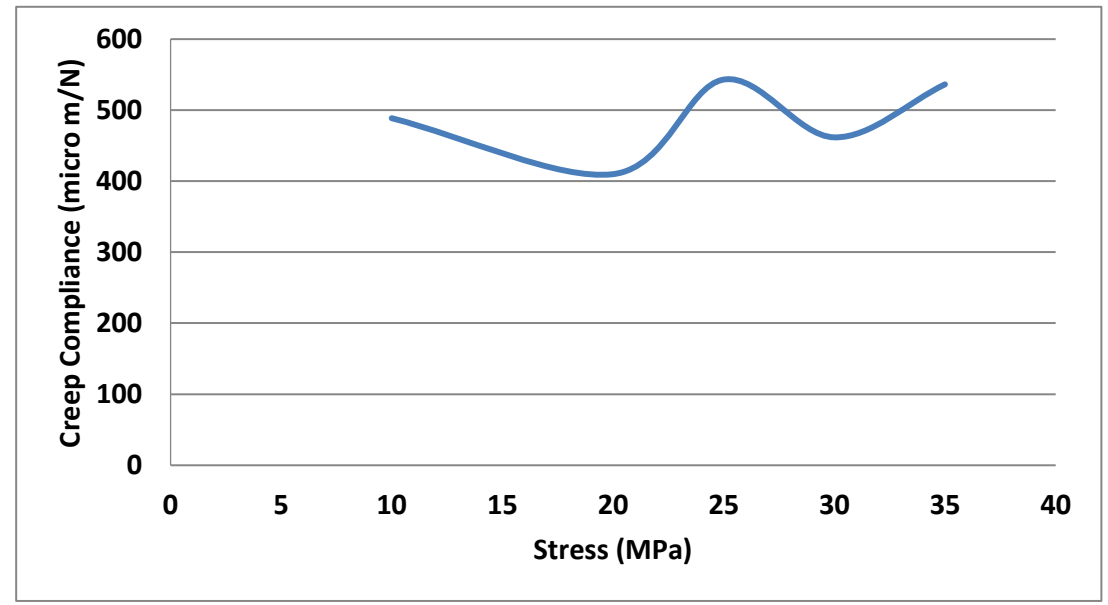

Figure 3-13 Recovery compliance versus stress curves of PLLA samples measured after 10 min of recovery. 


\section{Chapter 4 \\ Finite Element Approaches to Analyzing Helical Stent Expansion}

\subsection{Introduction}

Helical coil polymeric stents offer a plausible alternative to traditional metallic stenting methods; however, current understanding of their deployment, expansion and fixation behaviours remains limited. The goal of this work was to develop a finite element model to investigate the deformation characteristics of a helical bi-layer stent. The viscoelastic response was not considered since the strain rate during expansion was relatively slow and the material response was assumed to be primarily plastic.

The finite element method was used to investigate three approaches to modeling stent expansion with a focus on helical stent geometry, which differs from traditional wire mesh stent expansion. These three methods have been used in the literature of stenting to evaluate wire mesh stents and in this study we evaluate the applicability of these methods for non-traditional stents with helical geometry. These FEM strategies are: (i) displacement-driven expansion: to expand the stent with prescribed pressure or displacement at the inner surface of the stent (this is the simplest method); (ii) uniform expansion: place and radially expand a cylinder inside the stent, until it reaches an appropriate diameter; and (iii) stent-folded balloon expansion: position and inflate a folded balloon inside the stent, expanding it to the requisite diameter (this is the most complex method). Although each of the first two methods considered provided some insight into the helical expansion characteristics, these common displacement-controlled and uniform expansion methods were not able to demonstrate the characteristic local deformations observed in expansion. Furthermore, the relative efficiencies of these approaches differ based on computational complexity and, therefore, impact the information they can provide. Although computationally expensive, a coupled stent-balloon model had to be developed to demonstrate the observed non-uniform deformation. Recent advances in computing enabled us to extensively employ the most complex approach (i.e., stent-folded balloon) in this research. 
Flexibility of the helical stent was examined using a separate finite element model (Appendix A) where the stent was bent until the two ends of the coils touched each other. Then, once the constraints were removed, the stent returned to its original shape without any plastic or permanent deformation.

\subsection{Finite Element Model Development}

In this study, a typical helical stent was expanded using three common finite element expansion methods. The first two models (displacement-driven and uniform expansion) were solved using the implicit method (ABAQUS/Standard, Simulia) on a PC (Intel(R) core(TM) 2 Duo CPU, $3.00 \mathrm{GHz}$ ). The third model (coupled stent-folded balloon expansion) included complex contact interfaces between the stent and the balloon as well as between the folds of the balloon. In this case, the stent-balloon expansion model was developed and solved using an explicit finite element code (LS Dyna, LSTC) and a Symmetric Multiprocessing System (SMP) platform (AMD X86_64 GNU/Linux with 8 CPUs $(4 * 2.11 \mathrm{GHz})$ ). To ensure the results were comparable, the implicit and explicit methods were conducted under similar expansion cases.

\subsubsection{Material Properties}

In vitro material characterization studies (described in Chapter 3) showed that a preconditioning water-soaking step used to mimic body implantation conditions enabled required ductility level expansion. In this case, the mechanical contribution of the outer PLGA layer was negligible since it softened significantly under environmental conditions.

The measured mechanical properties from test condition iv (i.e., in $37{ }^{\circ} \mathrm{C}$ water), which most closely resembled the conditions during an actual stenting procedure, were used in the finite element model. Since the stiffness of the PLGA material under condition (iv) was negligible (Figure 3-4), this material was not included in the models. PLLA properties used in the model were: a multi-linear elastic-plastic with isotropic hardening material with a von Mises yield criterion, a modulus of $1.41 \mathrm{GPa}$, a Poisson's ratio of 0.3 , and a yield limit of $42.3 \mathrm{MPa}$. The yield limit was defined based on the proportional limit of the engineering stress-strain curve, which is widely accepted for polymers [Brinson, 2008]. The plastic region is defined in Table 4 1. Since the viscoelastic properties of the material were not considered, the elastic portion of the material properties of PLLA was modeled. PLLA characterization studies showed that this material exhibits plastic deformation as well. Therefore, an elastic-plastic material was used to 
represent this material in the modeling section of the study. Overall, for the scenarios considered in this study, the stent's viscoelastic response is expected to be secondary to its plastic response.

Table 4-1 Stress and plastic strain definition for the constitutive model.

\begin{tabular}{|l|l|l|}
\hline & Yield stress ( MPa) & Plastic strain \\
\hline 1 & 42.3 & 0.000 \\
\hline 2 & 43.9 & 0.008 \\
\hline 3 & 44.7 & 0.306 \\
\hline 4 & 46.7 & 1.000 \\
\hline
\end{tabular}

\subsection{Displacement driven expansion}

It was found that applying prescribed radial displacement to the inner surface of the stent provides the simplest method for uniform expansion (Figure 4-1). The stent model was fixed at one end to prevent rigid body motion and the radial expansion boundary condition allowed for expansion, uncoiling and a corresponding reduction in length.

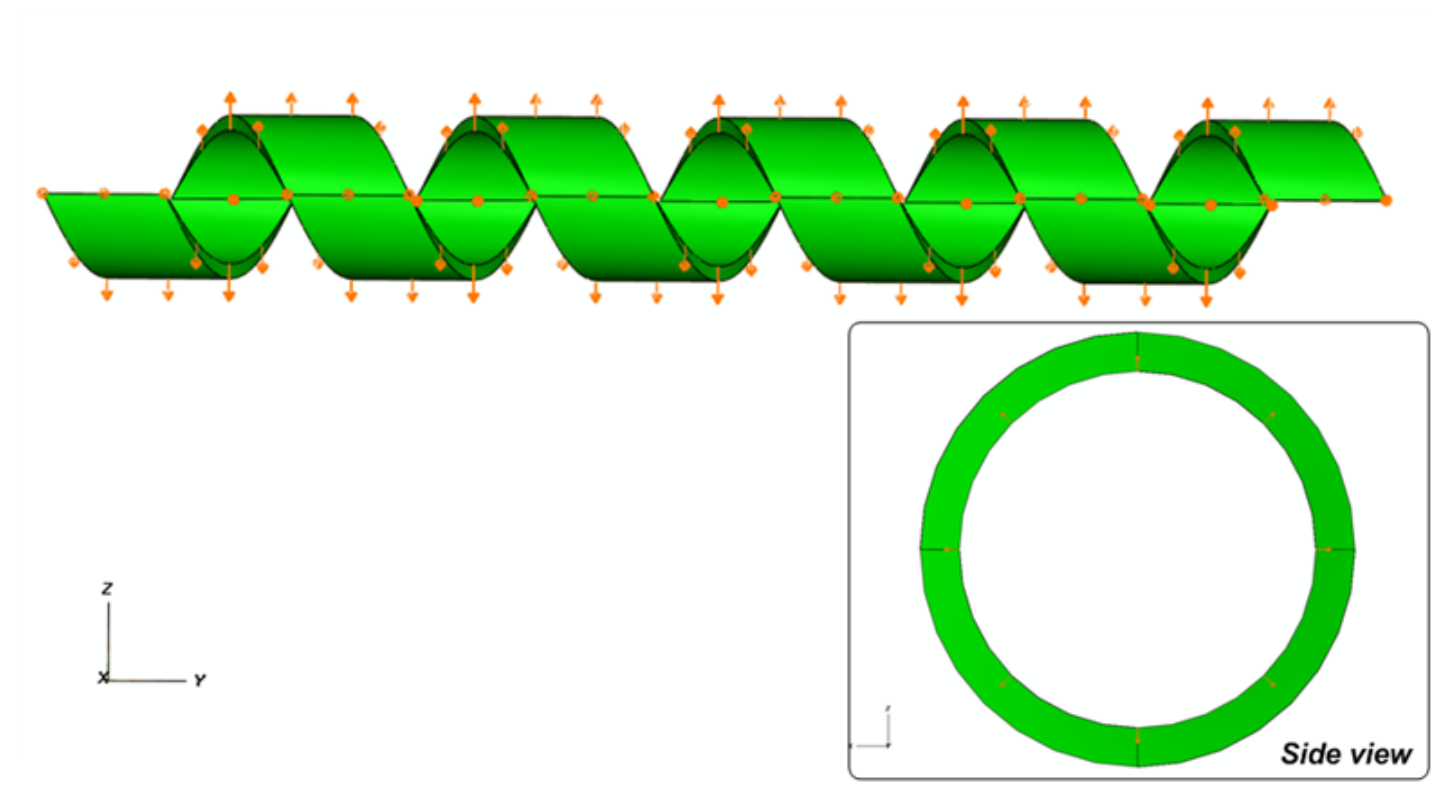

Figure 4-1 Prescribed radial displacement on the inner surface of a five-coil stent, arrows denote boundary conditions. 
In FE modeling of wire mesh stent expansion, it is common to apply a prescribed radial displacement or pressure to the inner surface of the stent [Bedoya, 2006; Rogers, 1999; Dumoulin, 2002; McGarry, 2004; Paszenda, 2005; Mori, 2005; Martin, 2002]. However, radial expansion of helical stents is the result of uncoiling and a decrease in the pitch value of the stent.

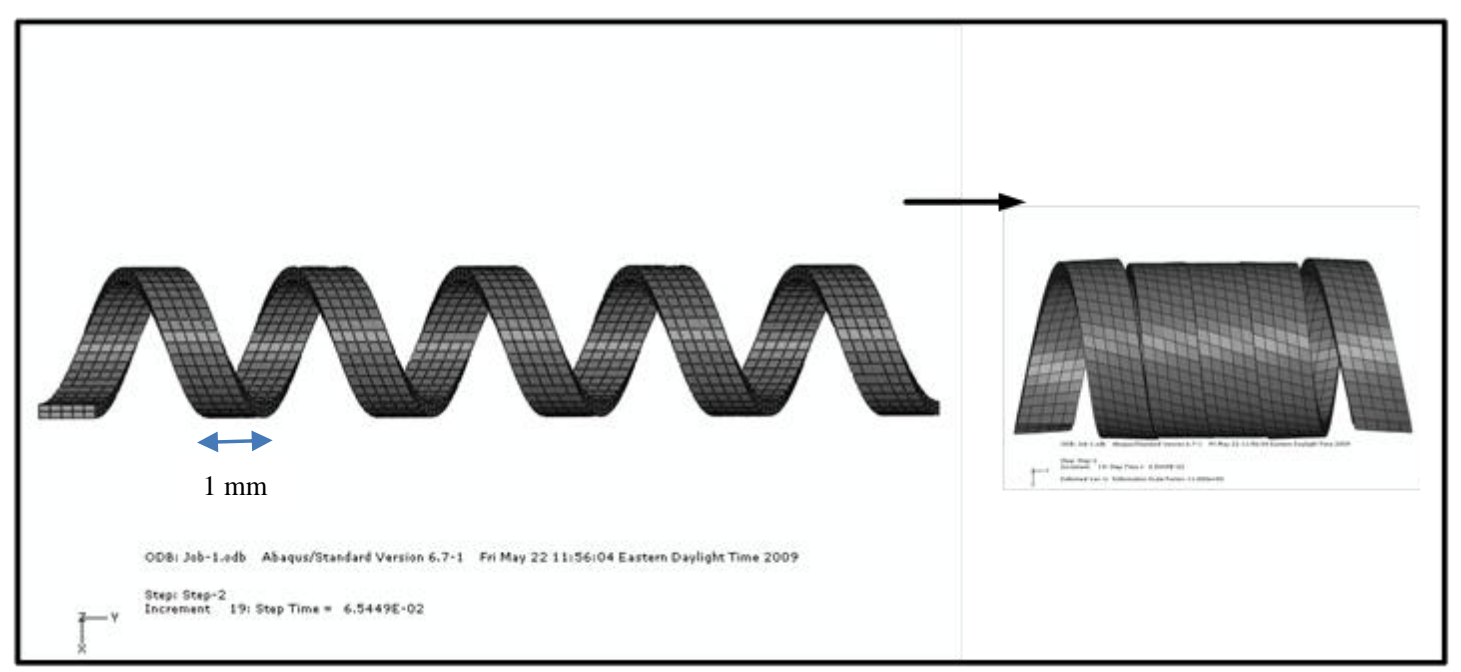

Figure 4-2 Predicted helical stent expansion using applied radial displacement.

To build the displacement-driven expansion model shown Figure 4-1, axial contraction of the helical stent was not restricted, enabling a decrease in the stent pitch value and a concomitant increase in stent diameter (the first helical stent expansion mechanism, Figure 2-3). The model was meshed with 8-node linear brick, reduced integration elements. A convergence study was also conducted to find the suitable element size. After 0.1 millimeters of radial expansion, the coils were in contact with each other and plastic deformation was predicted at the contact locations due to the coil interaction. The model subsequently terminated due to excessive element distortion (Figure 4-3). It took 2 minutes to solve this model up to the termination point using an implicit finite element solver (Abaqus, Simulia). 


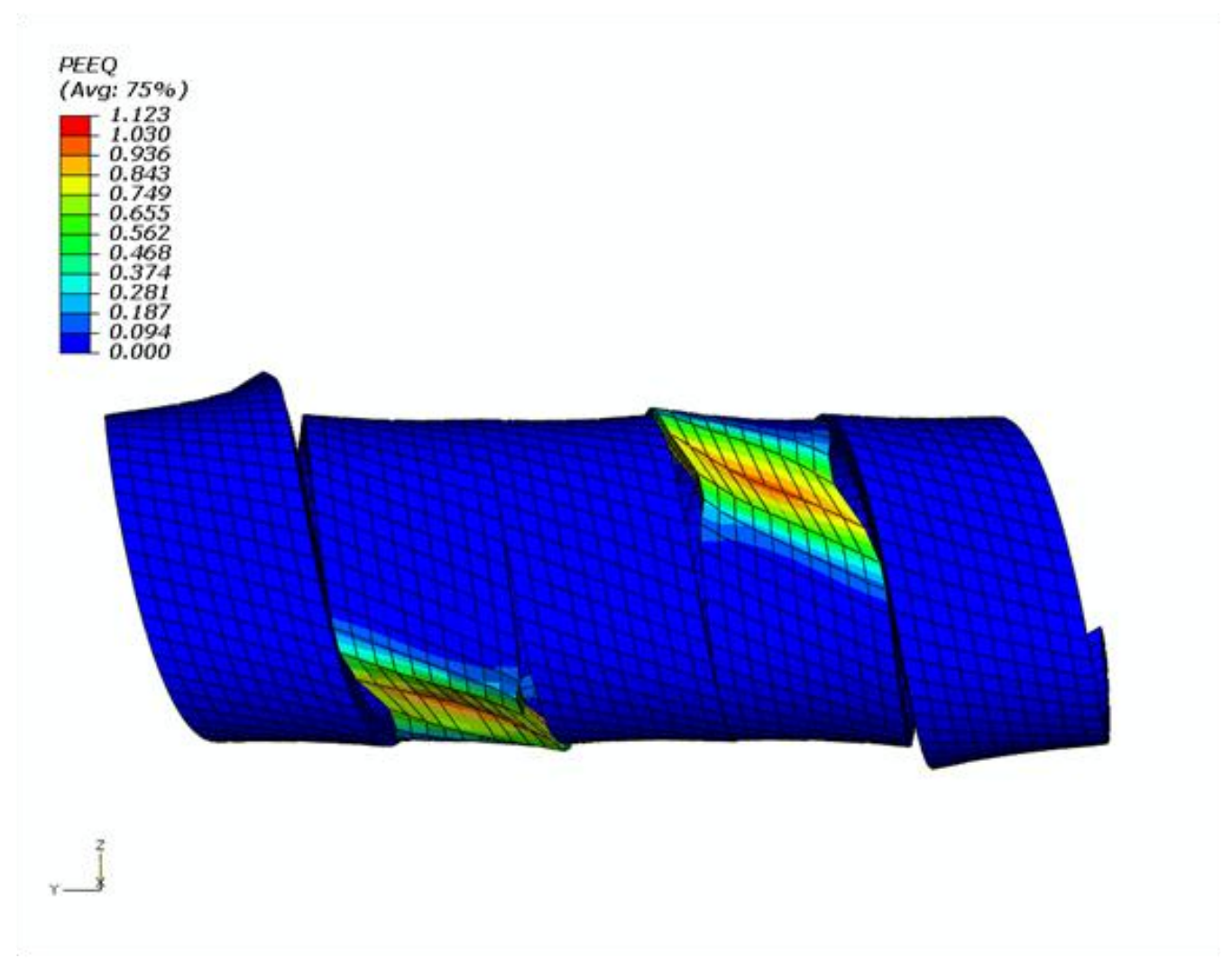

Figure 4-3 Equivalent plastic strain for the displacement-driven expansion at model termination $(0.054 \mathrm{~mm}$ radial expansion).

Using the simple displacement driven expansion method, radial expansion of the helical stent occurs due to uncoiling and a decrease in the stent's pitch value. By applying prescribed displacement or pressure directly to the inner surface of the stent, the stent tended to decrease in pitch value in order to expand in the radial direction, due to the absence of axial constraints (Figure 4-2). This non-physical result was due to the lack of interaction between the stent and an expansion medium. A cylinder was used to uniformly expand the stent.

\subsection{Uniform expansion}

Helical stent expansion and recoil behaviour was studied, first by uniformly expanding the stent to the required diameter using a high stiffness cylinder and then allowing it to recoil. The model was validated using experimental test data measured in vitro. Later, a parametric study 
was undertaken to investigate the effect of stent geometry and the coefficient of friction (COF) at the stent-cylinder interface on the stent's expansion and recoiling characteristics.

\subsubsection{Stent and Cylinder Geometry}

An eight-coil helical stent geometry, with an inner radius of $0.55 \mathrm{~mm}$, thickness of $0.12 \mathrm{~mm}$, and pitch value of $0.12 \mathrm{~mm}$ (Figure 4-4), was used to develop a commercial implicit finite element code (ABAQUS/CAE 6.7-1).

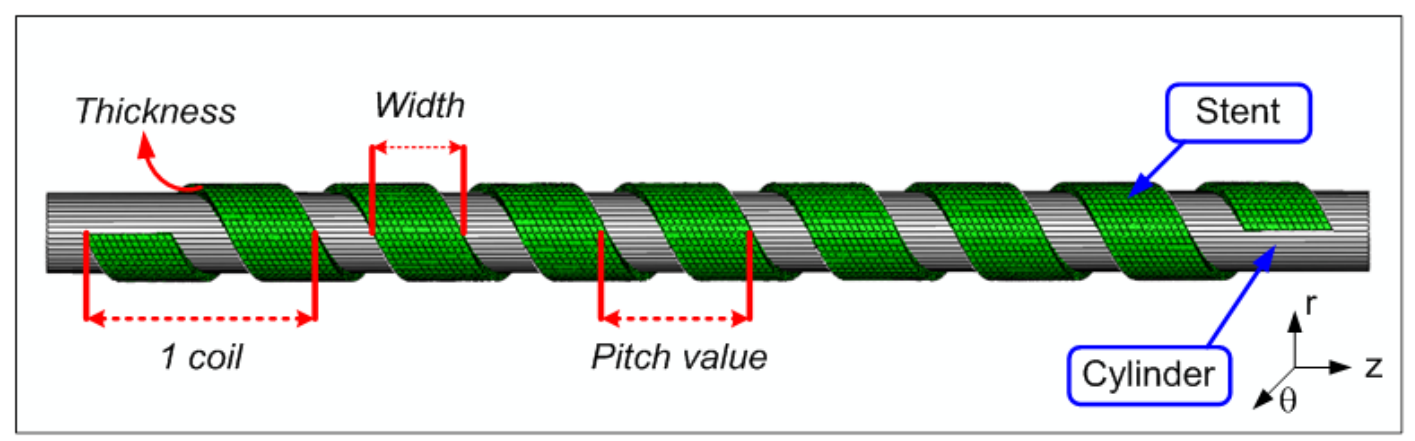

Figure 4-4 Eight-coil helical stent geometry.

A deformable cylinder, with an initial outer diameter of $1 \mathrm{~mm}$, was integrated into the stent model. Although used primarily to provide radial expansion, the cylinder was modeled as deformable to enable contact and friction between the stent and cylinder. This model was solved using an implicit finite element code (ABAQUS/ Standard, Simulia). An automatic contact algorithm was applied at the interface of stent and the cylinder with a coefficient of friction (COF) of 0.1, consistent with a typical Nylon 12 balloon [Vad, 2010] . The cylinder was constrained to expand only in the radial direction with no other boundary conditions applied to the stent. All contacts were modeled using a penalty-based formulation. For the recoil study, the contact between the stent and the cylinder was disabled following expansion to the desired diameter. The cylinder was modeled as elastic with a Young's modulus of $500 \mathrm{GPa}$, which is much higher than the stiffness of the stent. Therefore, deformation of the stent did not cause significant deformation of the cylinder. The expanding cylinder was modeled with an outer diameter of $1 \mathrm{~mm}, 14 \mathrm{~mm}$ length and a thickness of $0.1 \mathrm{~mm}$. The stent was expanded to an outer diameter of $3 \mathrm{~mm}$, including a constant thickness of $0.08 \mathrm{~mm}$ for the PLGA layer. 


\subsubsection{Convergence study for uniform expansion model}

For the uniform expansion model, the stent and the expanding cylinder were meshed with Solid (Linear Hexahedron C3D8R) and Shell (Linear quadrilateral S4R) elements, respectively. The elements are considered robust, in terms of convergence, for difficult contact problems [Mortier, 2008; De Beule, 2008; Lim, 2008]. The PLLA layer of the stent had four elements through the thickness, to capture the bending effects. It was important that the number of elements in the circumferential direction were same in both the stent and the cylinder (44 elements in this case) for contact requirements.

The sensitivity of the results to the size of elements was investigated. Changing the size of the elements from $1.13 \mathrm{e}-3 \mathrm{~mm}^{3}$ to $5.64 \mathrm{e}-4 \mathrm{~mm}^{3}$ changed von Mises stress by $14 \%$. By continuously decreasing the element size to $2.82 \mathrm{e}-4 \mathrm{~mm}^{3}$, the predicted von Mises stress converged (Figure 45), corresponding to 4 elements through the thickness, 44 elements around the coil circumference, and 12 elements along the width of each coil. Based on the converged mesh density, the 8-coil stent, with thickness of $0.12 \mathrm{~mm}$ and width of $1.2 \mathrm{~mm}$, was meshed with 16896 solid elements.

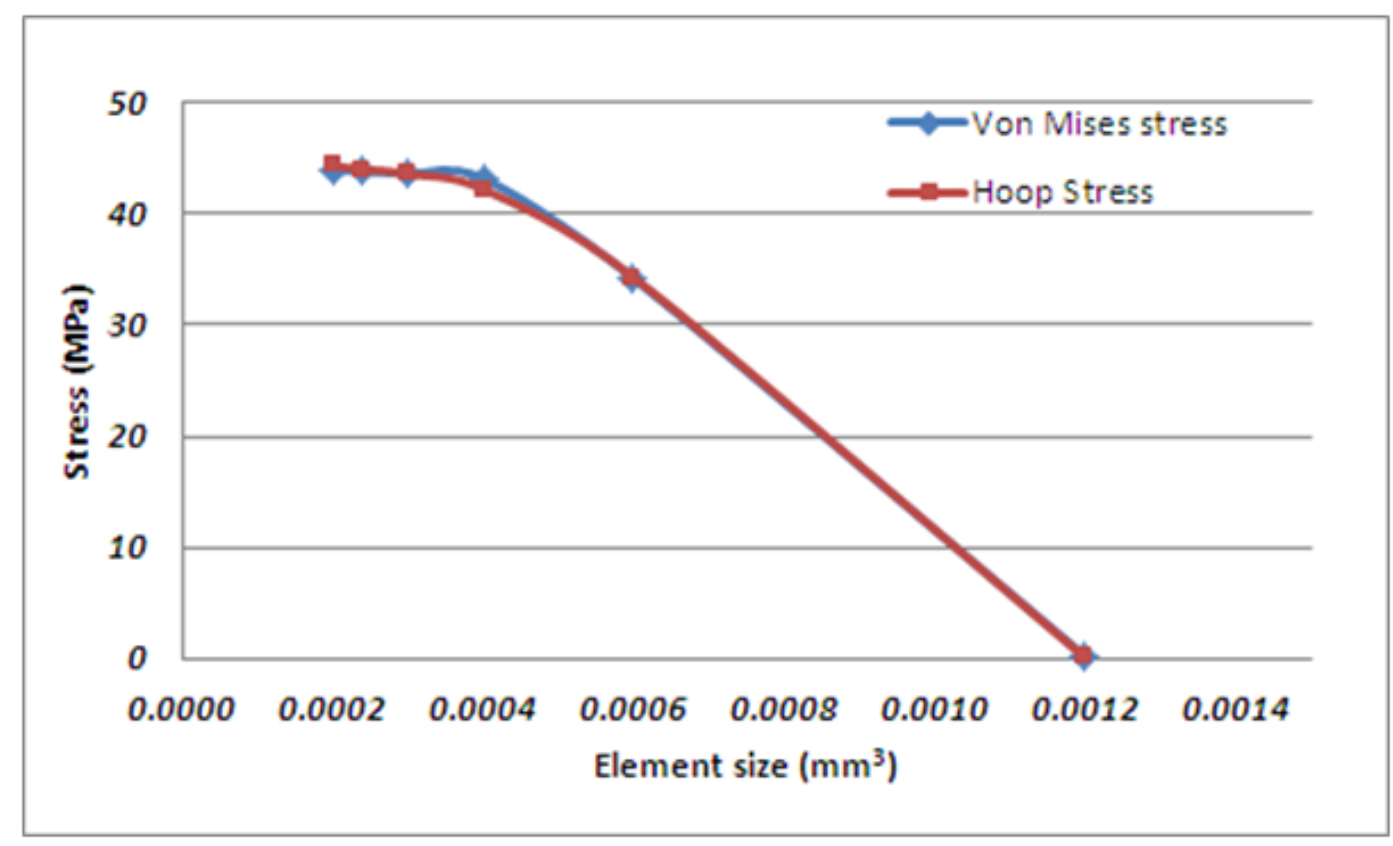

Figure 4-5 Convergence study based on maximum stress values, element size quoted as single element volume. 
The sensitivity of the results to the size of elements was investigated using the maximum equivalent plastic strain and maximum principal strain. The same convergence trend was observed for both strain measures (Fig 4-6).

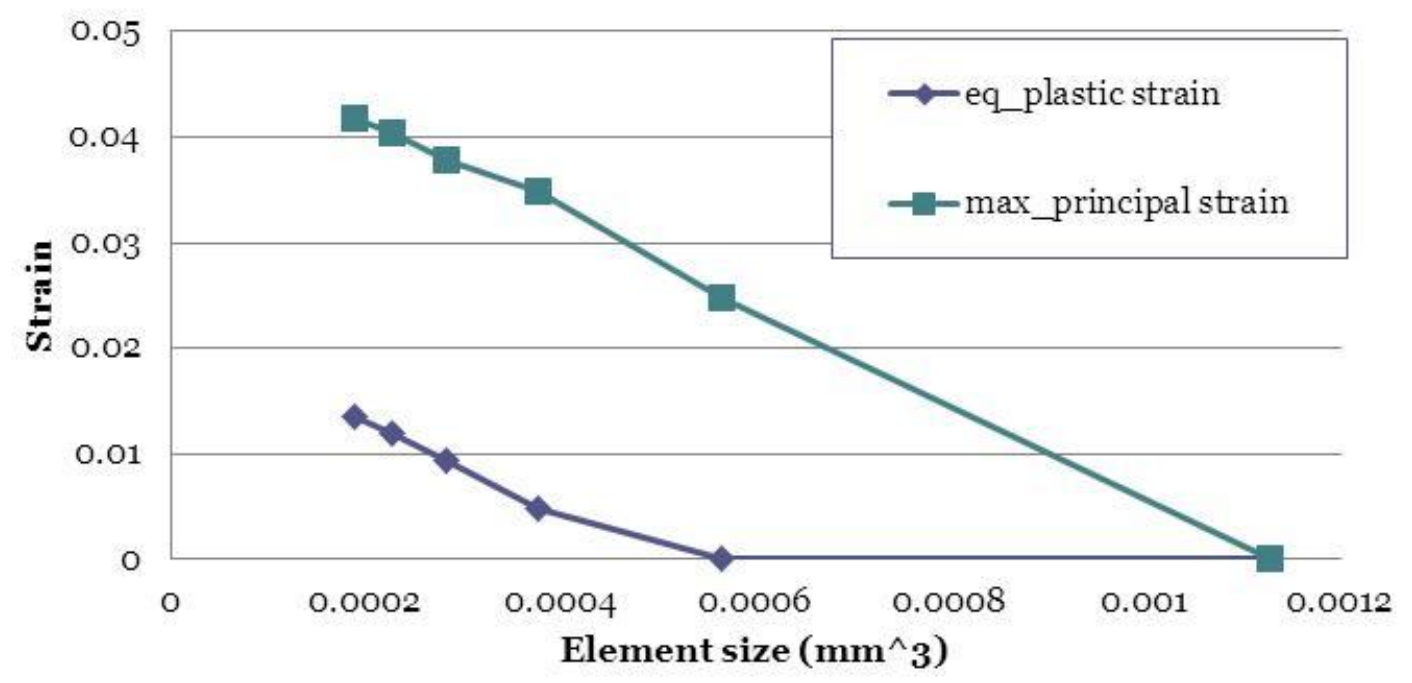

\section{Figure 4-6 Convergence study based on maximum equivalent plastic strain and maximum principal strain, element size quoted as single element volume.}

The implicit quasi-static process was considered suitable for this application for several reasons: the expansion time of a typical polymeric stent is on the order of 3-7 minutes; the materials considered have a relatively low density; and inertia was assumed to have a negligible impact on the stent expansion.

\subsubsection{Model Validation}

The model was validated using experimental stent expansion and radial recoiling measurements taken in vitro by the NTU research group. During the experiment, an 8.5-coil stent with a pitch value of $2.0 \mathrm{~mm}$, width of $1.2 \mathrm{~mm}$, an inner layer of PLLA (0.12 mm thick), and outer layer of PLGA $\left(0.08 \mathrm{~mm}\right.$ thick) was preconditioned in $37^{\circ} \mathrm{C}$ water for $30 \mathrm{~min}$ before expansion. The material properties measured following this preconditioning step were incorporated in the model. The stent was then expanded using a folded balloon from an inner diameter of $1 \mathrm{~mm}$ to an outer diameter of $3 \mathrm{~mm}$ in $37^{\circ} \mathrm{C}$ water. Immediately following expansion, the stent length was reduced to $13 \mathrm{~mm}$ (foreshortening) and a total of 4 coils (uncoiling). A similar stent (8coils, $18.2 \mathrm{~mm}$ length, $1 \mathrm{~mm}$ ID, $1.2 \mathrm{~mm}$ width, $2 \mathrm{~mm}$ pitch) was evaluated using 
the finite element method. The numerical stent was expanded to an outside diameter of $3 \mathrm{~mm}$ under uniform expansion. The model predicted that the stent would shorten to $13.56 \mathrm{~mm}$ with a total of 3.9 coils (Figure 4-7) (compared to $13 \mathrm{~mm}$ length and 4 coils in the experiment). Since foreshortening and uncoiling are two semi-independent parameters (considering the effect of the pitch angle), this provided a good assessment of model performance. The change in outer diameter of the stent with increasing balloon pressure was also measured experimentally (Figure 4-8).

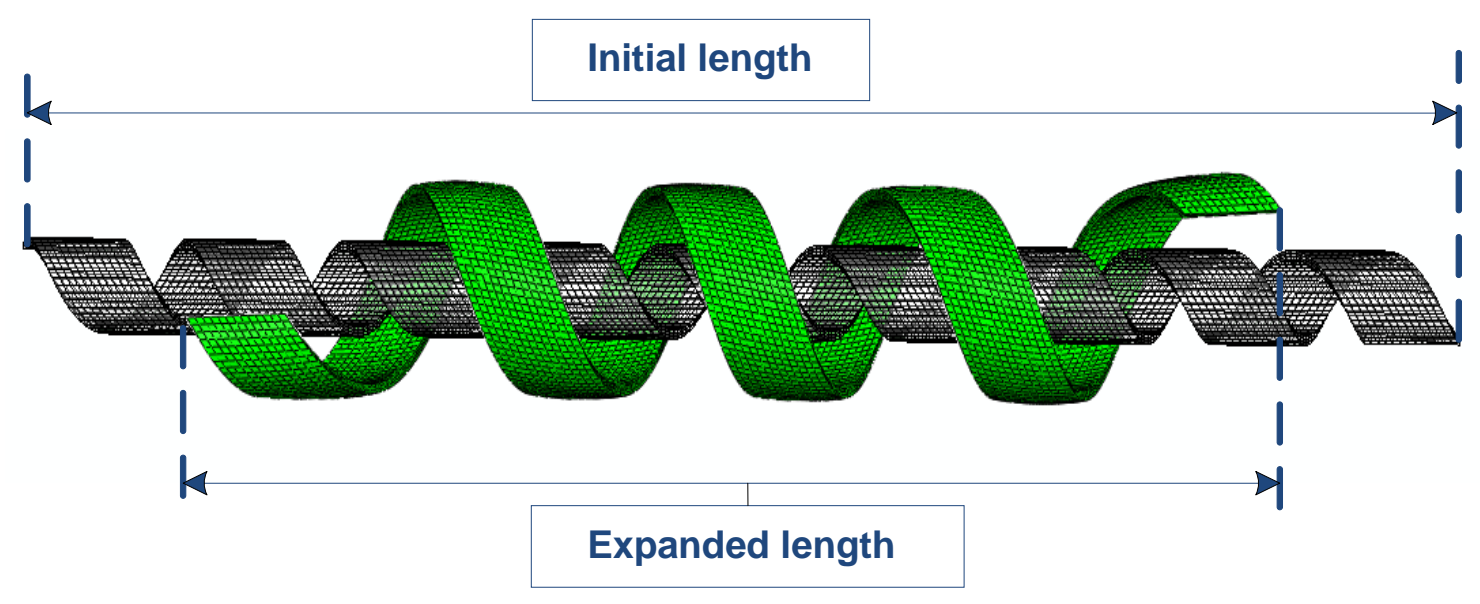

Figure 4-7 Unexpanded and expanded stent showing foreshortening and uncoiling. 


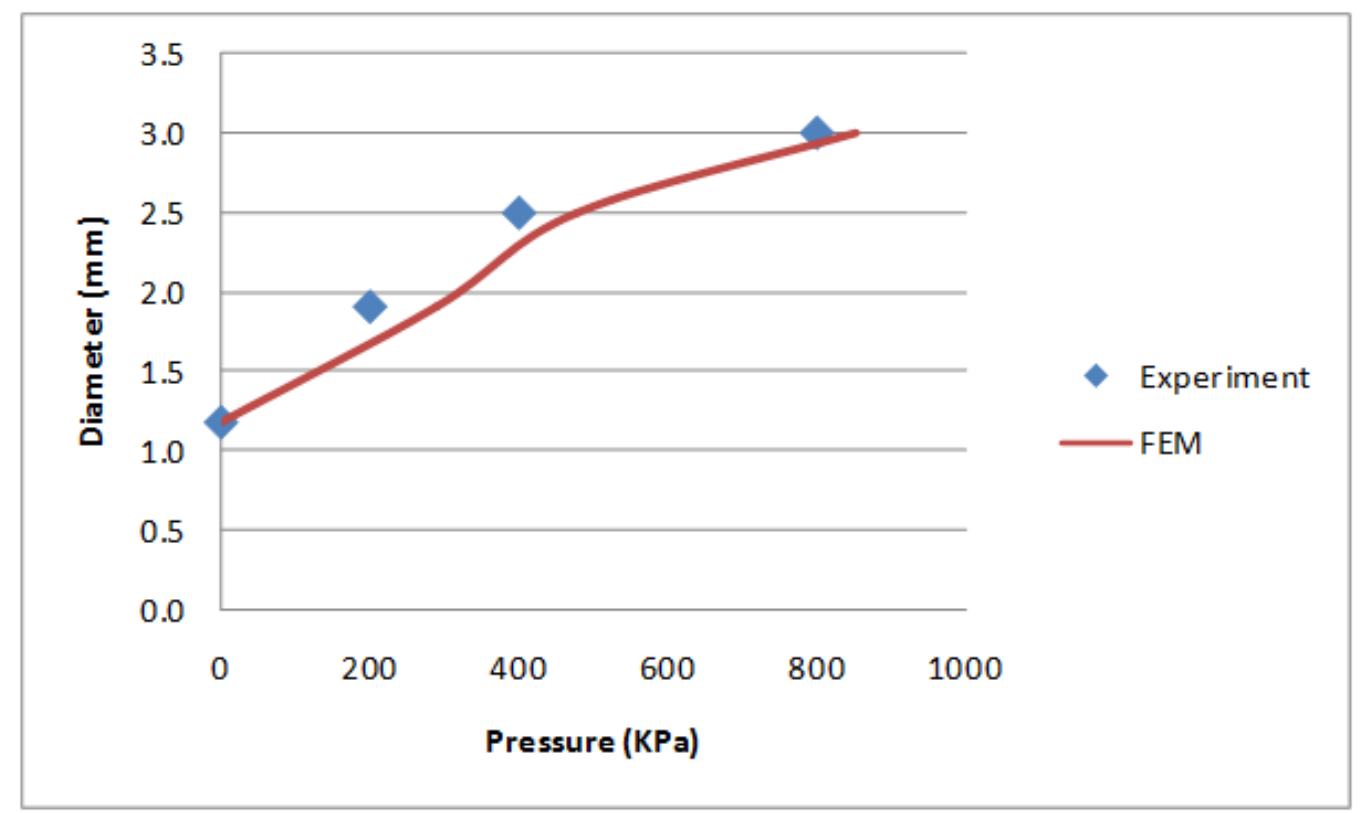

Figure 4-8 Pressure versus diameter for expansion of an 8.5 coil stent.

The stent outer diameter (OD) decreased to $2.5 \mathrm{~mm}$ (2.1 mm ID) (Figure 4-9) when the balloon was deflated. In all cases, the model predictions were in good agreement with the experimental results provided by NTU.

An eight-coil stent model with a width of $1.2 \mathrm{~mm}$, thickness of $0.12 \mathrm{~mm}$ was expanded to an inner diameter of $2.6 \mathrm{~mm}$ (effective outer diameter of $3.0 \mathrm{~mm}$ ) and allowed to recoil (Figure 4$10)$.

The uniform expansion method allowed for complete expansion of the helical stent (Figure 410) with the pressure-radius curve following expected trends (Figure 4-8), taking approximately 90 minutes to solve using an implicit finite element solver (Abaqus, Simulia). 


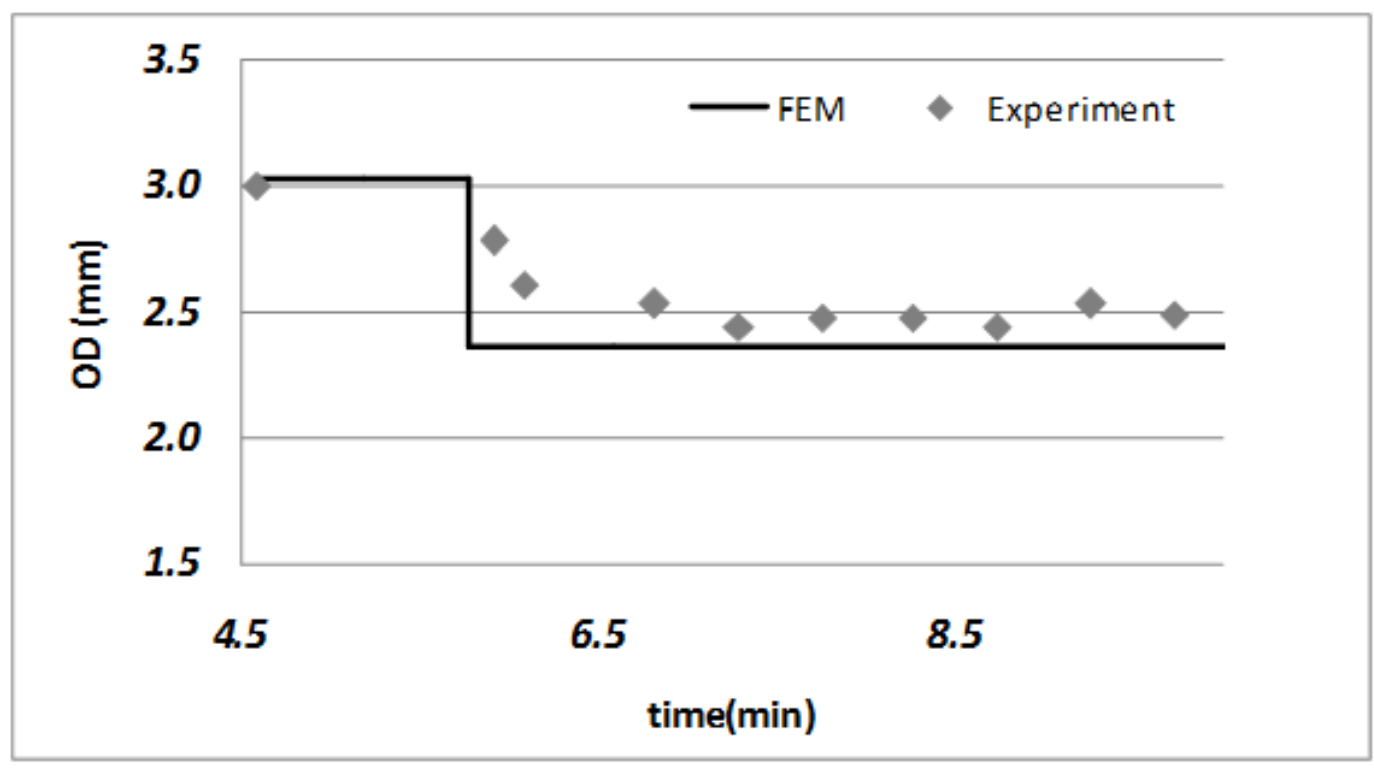

Figure 4-9 Stent recoiling (outside diameter) following expansion and removal of balloon.

It should be noted that viscoelastic effects were not included in the model; however, these effects are expected to be secondary contributors to the response, which is dominated by plasticity in the material at the very low strain rates encountered during expansion. One of the most significant challenges in developing this model was the contact conditions between the expansion cylinder and stent. In general, high contact stiffness caused the stent to detach from the expansion cylinder, while low contact stiffness tended to cause interpenetration at the interface and limited uncoiling of the stent. The contact stiffness ( $365 \mathrm{kN} / \mathrm{mm}$ for an 8-coil stent) was determined by monitoring the stress in the end coils of the stent, which are relatively unconstrained and subject to simple bending stresses. The circumferential stress in these coils was compared with calculated values using the Winkler curved beam theory [Hibbeler, 1999], which was valid for small strains. The results of uniform circumferential expansion of a one-coil stent from the inner diameter of 0.5 to 0.52 were compared with the stress results based on the Winkler theory. The maximum stresses have $10 \%$ error, caused by friction at the stent-cylinder interface in the finite element model. 


\subsubsection{Predicted Stress and Strain Resulting from Expansion}

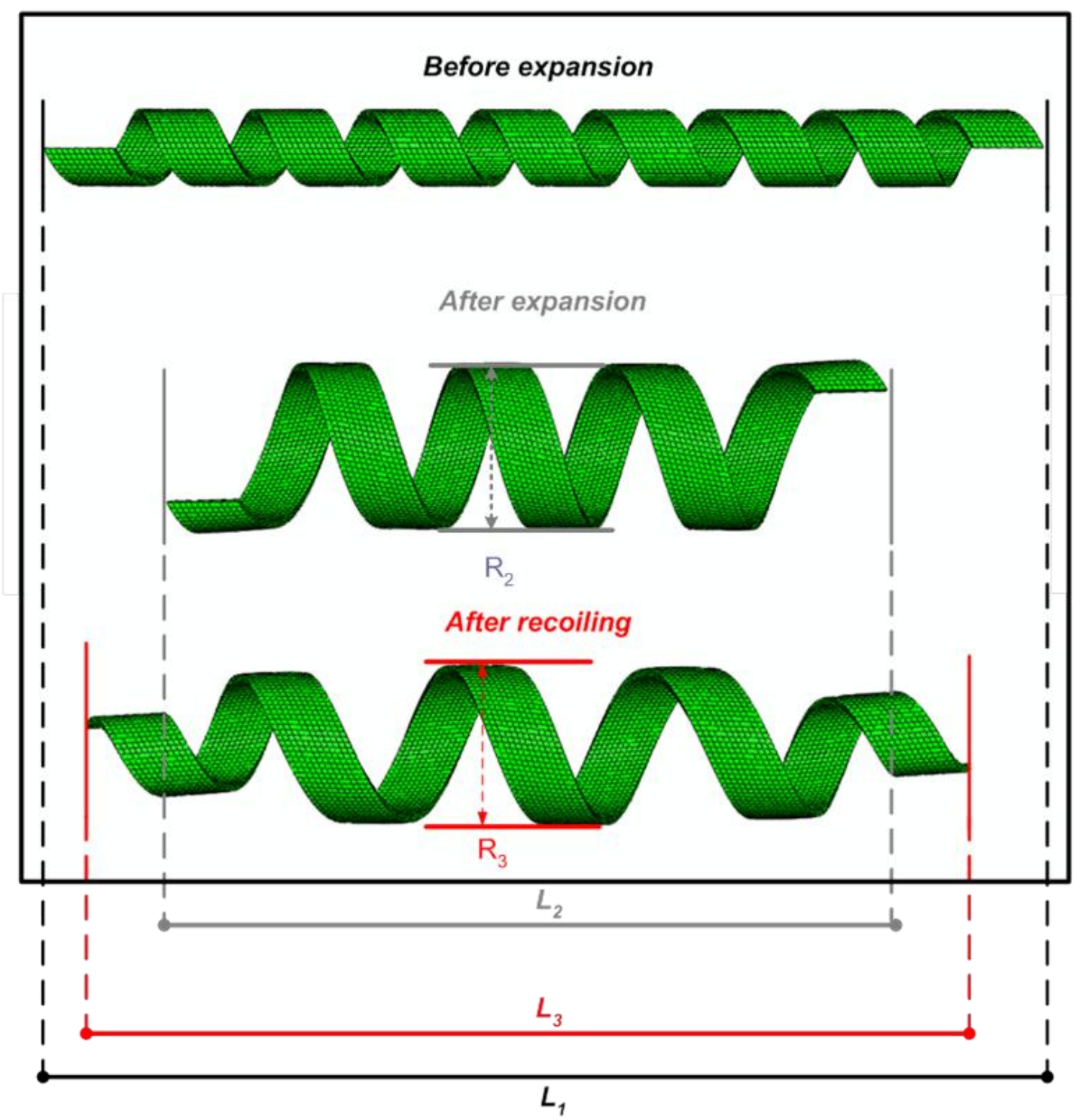

Figure 4-10 Eight-coil stent model before expansion, after expansion and after recoiling.

As the stent's diameter expands to $R_{2}$ its length shortens to $L_{2}$, and after recoiling the diameter decreases to $R_{3}$ and the length increases to $L_{3}$. The actual magnitude of the numbers depends on the stent design and expansion conditions, as will be described in the next sections. 
Circumferential bending is the dominant deformation process during expansion and recoiling, which can be evaluated using the circumferential stress predicted in the stent. The circumferential stress experienced by an 8-coil stent during expansion recoiling is compressive at the outer surface and tensile at the inner surface (Figure 4-11, Figure 4-12, and Figure 4-13). The stress distribution of the stent is three dimensional after recoiling. Unlike a rectangular beam, which is subject to bending, stent coils are subject to axial stress from the increase in pitch during uncoiling. However, the plastic deformation in the axial direction limits part of the stent's axial movement so that the elastic circumferential stress cannot be entirely released during recoiling and the inner coils of the stent are under tension. This fact is more obvious in the expansion model that describes prescribed radial displacement at the stent's inner surface. Since axial resistance is not available in this model, the coils start to overlap and collapse together during radial expansion. Balloon-expandable stents are usually anchored using plastic deformation, where the equivalent plastic strain was higher at the inner surface of the coils (Figure 4-14).

Following expansion of the stent, both radial and axial deformations were observed. Axial stress caused the stent to bend along the width of each coil. The three-dimensional stresses produced a locking force, which caused the circumferential stress in the coils to decrease to zero after recoiling. 


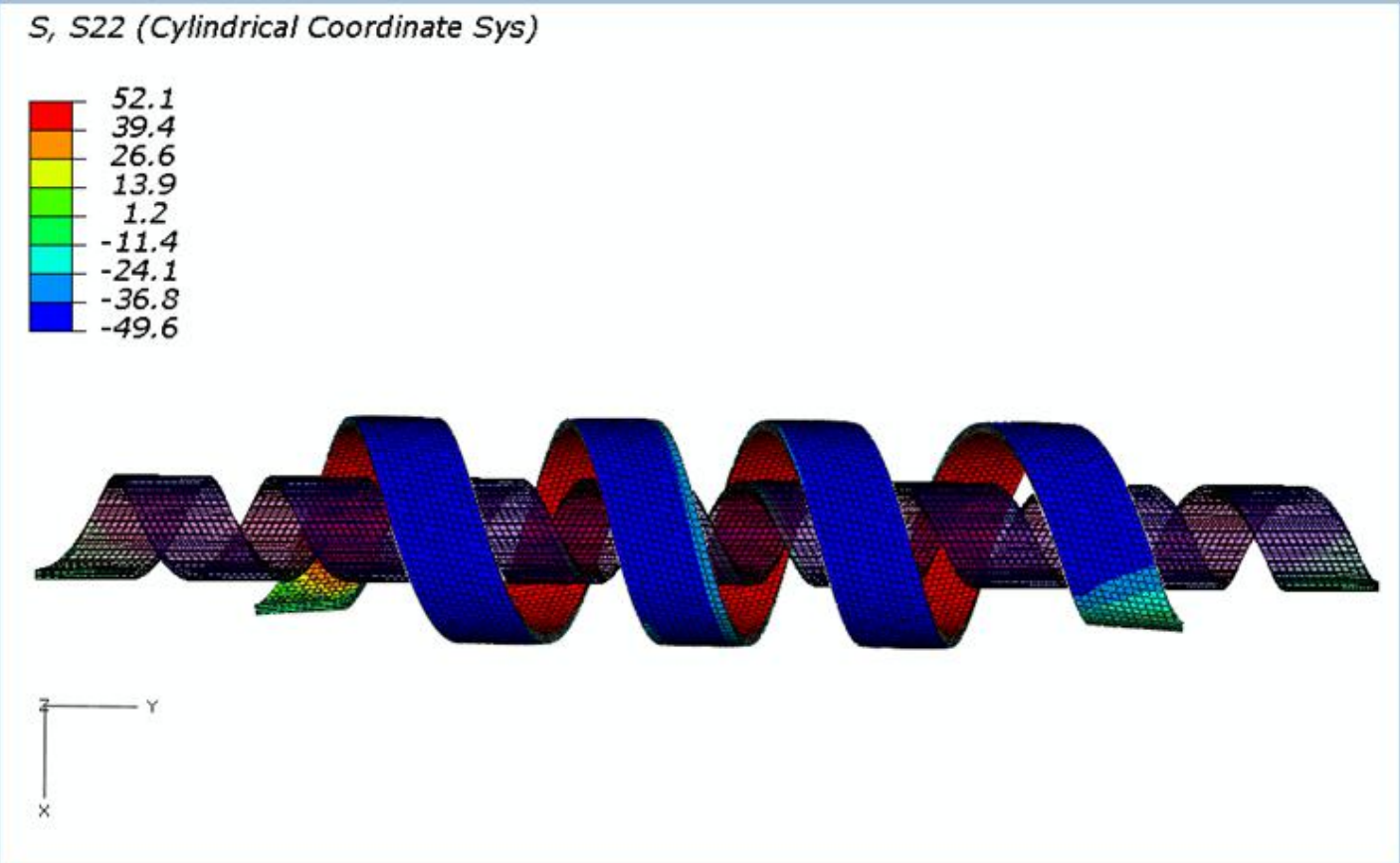

Figure 4-11 Circumferential stress distribution (in MPa) for an 8-coil stent after expansion.

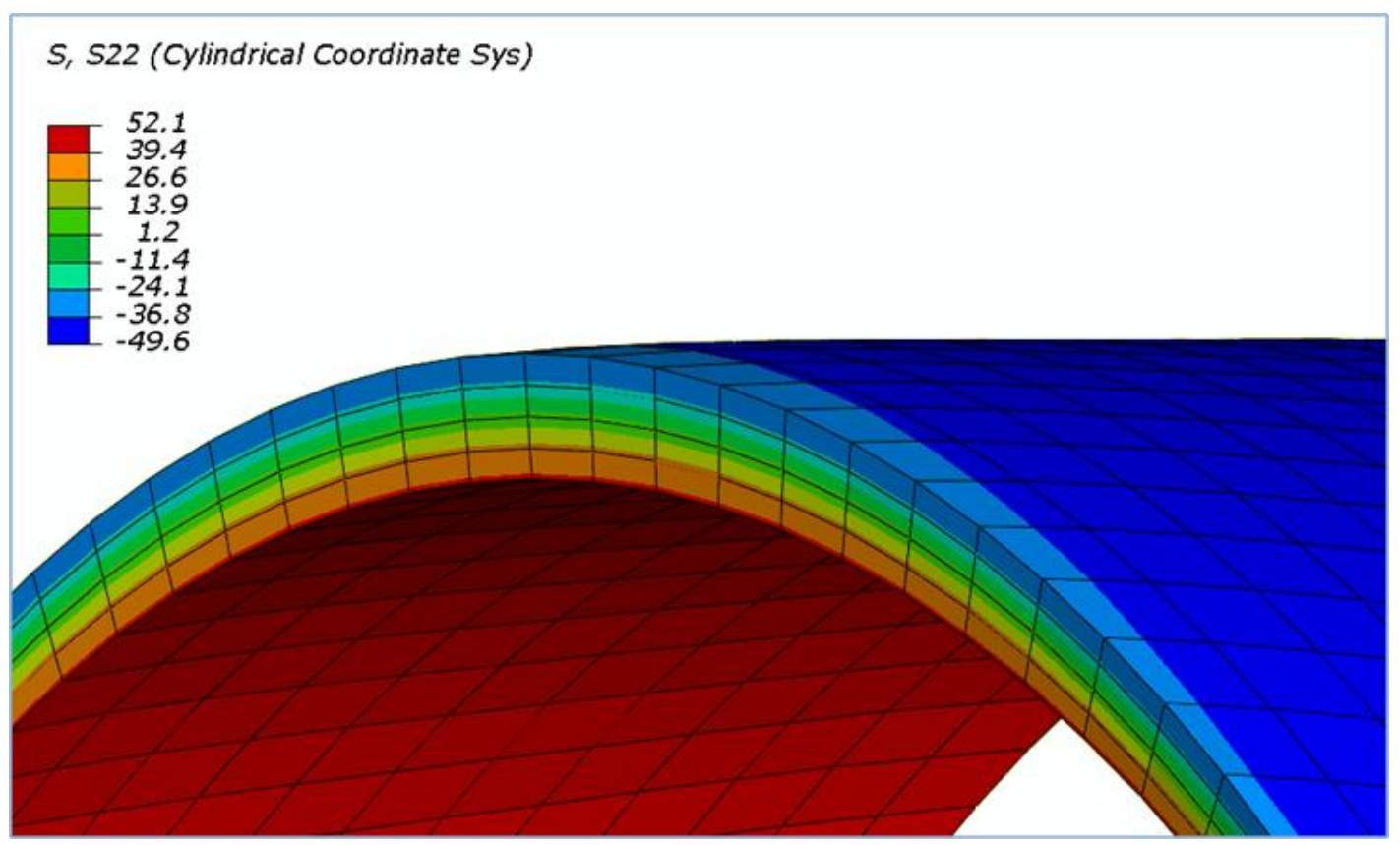

Figure 4-12 Circumferential stress distribution (in MPa) for an 8-coil stent after expansion along the thickness of the inner coil. 


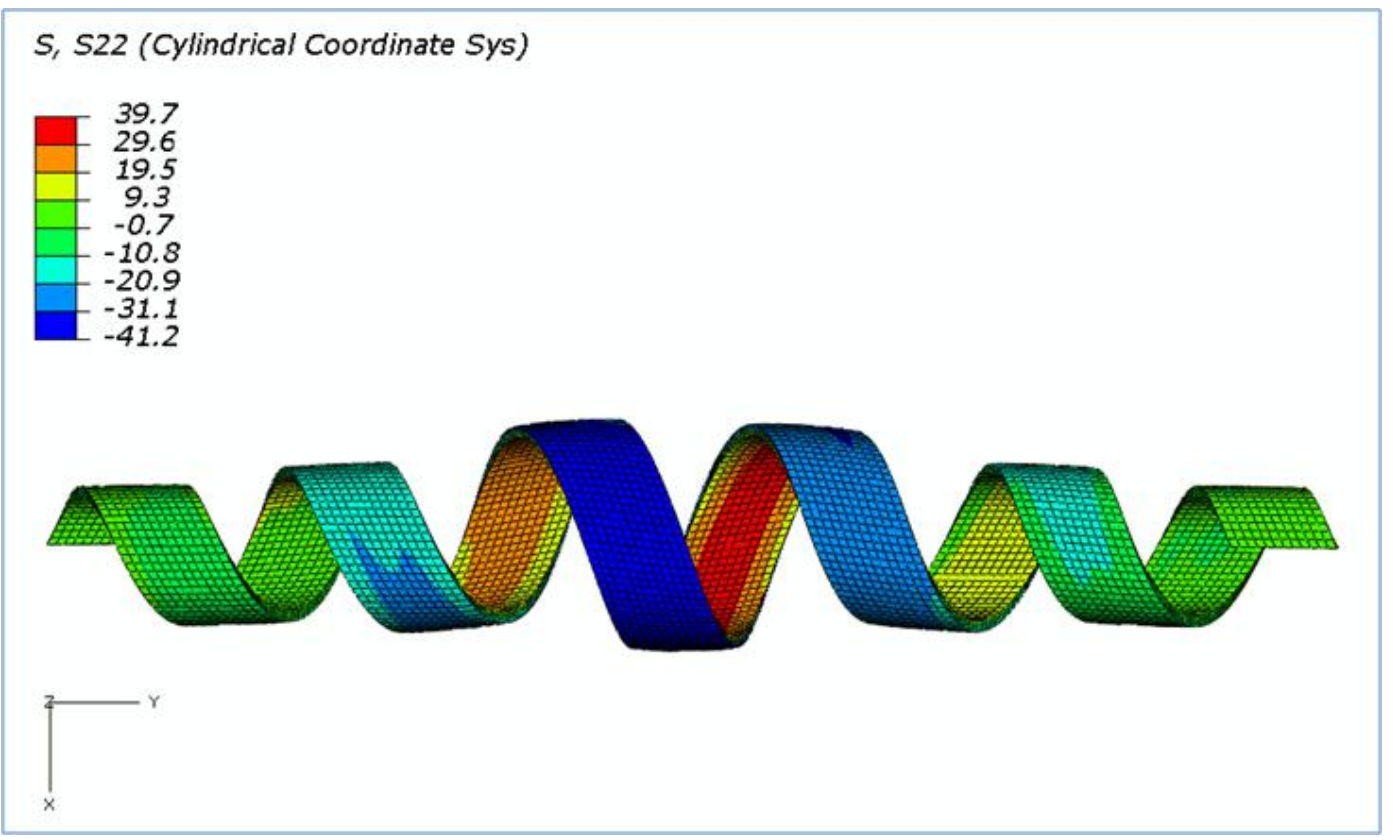

Figure 4-13 Circumferential stress distribution (MPa) for an 8-coil stent after recoiling.

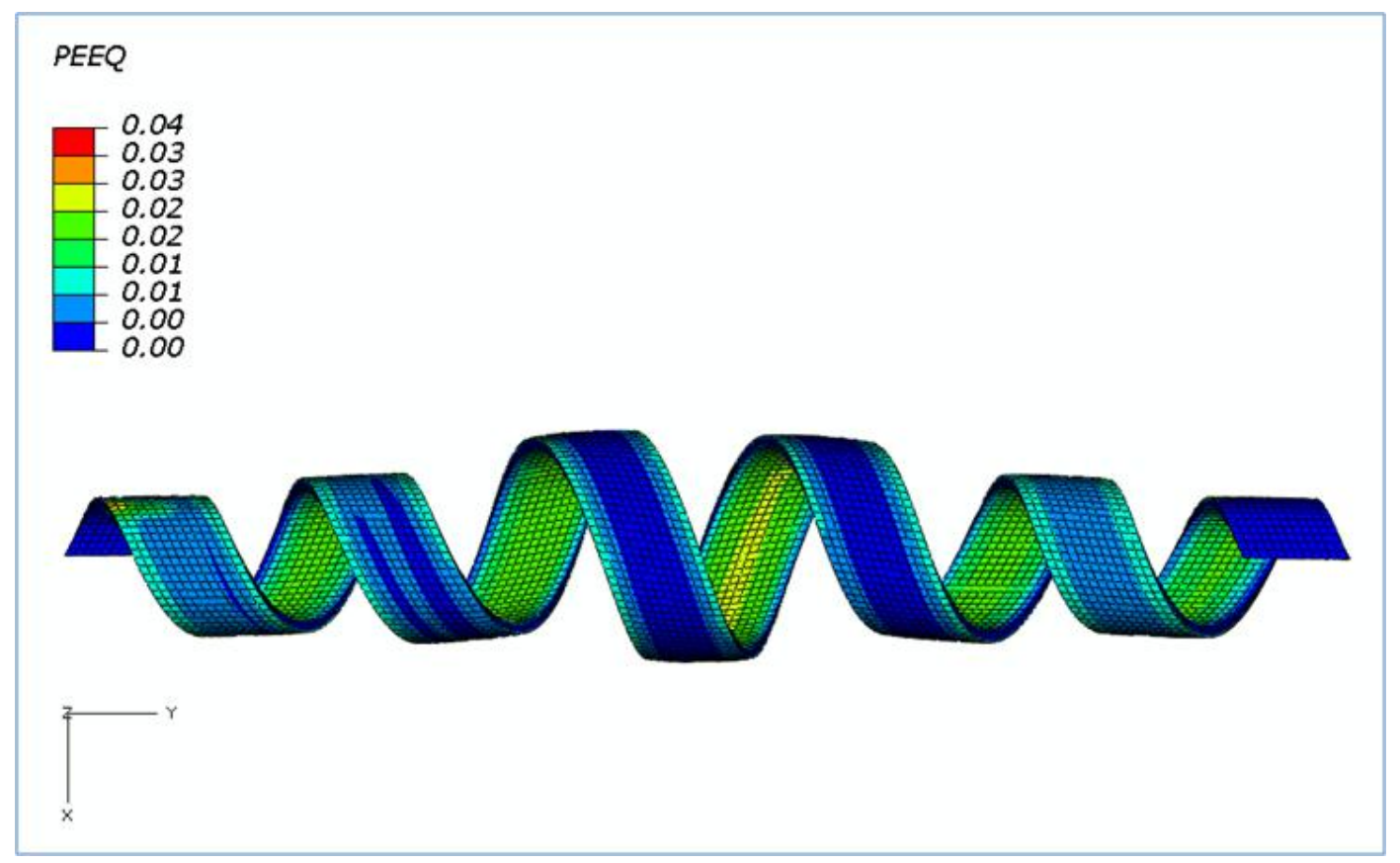

Figure 4-14 Equivalent plastic strain distribution for an 8-coil stent after recoiling. 


\subsubsection{Two-layer versus one-layer stent expansion}

To evaluate the effect of PLGA layer on expansion of a bi-layer helical stent, the PLGA layer (3 elements through the thickness) was included in a 3-coil stent, and expanded to an outer diameter of $3 \mathrm{~mm}$. The PLGA layer had a thickness of $0.08 \mathrm{~mm}$, Young's modulus of 0.001 $\mathrm{MPa}$, and Poisson's ratio of 0.3 (Figure 4-15). The outer PLLA surface and the inner PLGA surface were tied together, as would be the case in the actual stent. For comparison, a PLLAonly stent was modeled (Figure 4-16).

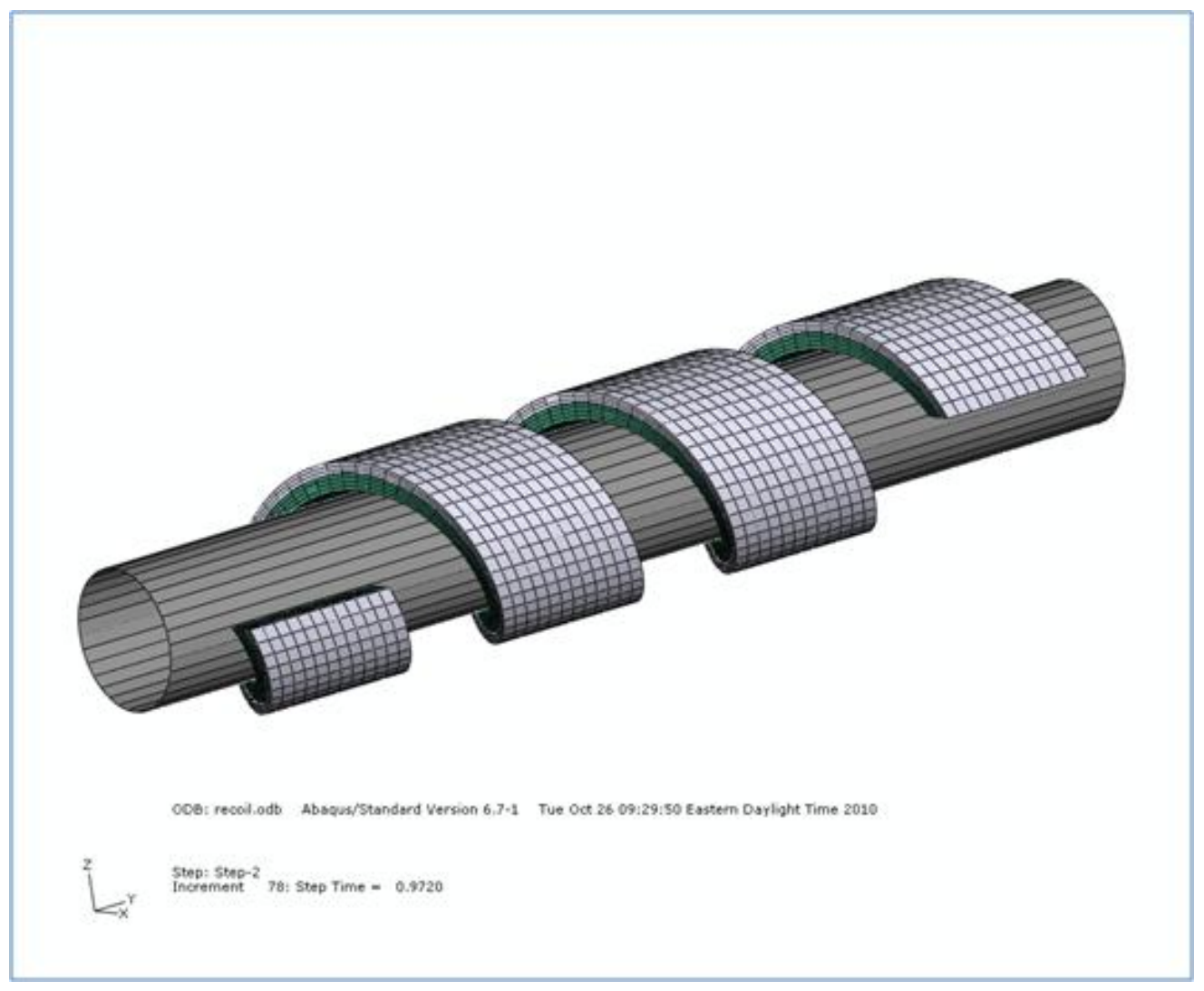

Figure 4-15 Two-layer stent including PLLA (inner layer) and PLGA (outer layer) layers. 


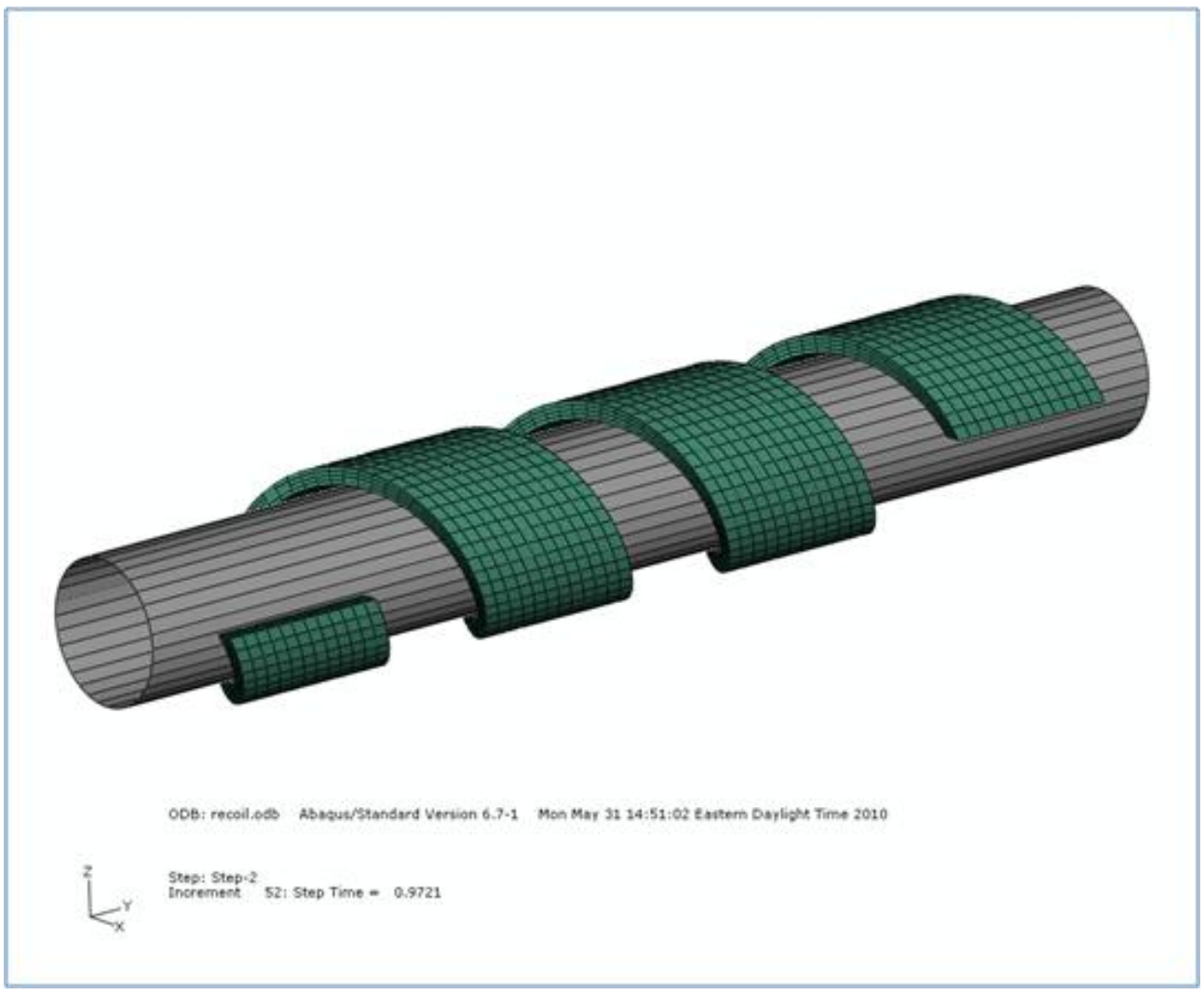

Figure 4-16 One-layer PLLA stent model.

As shown in Figure 4-17, the two models predicted identical von Mises stress results in the PLLA layer, suggesting the PLGA layer did not influence the outcome of the model. This result was also predictable based on the material properties of PLGA. 


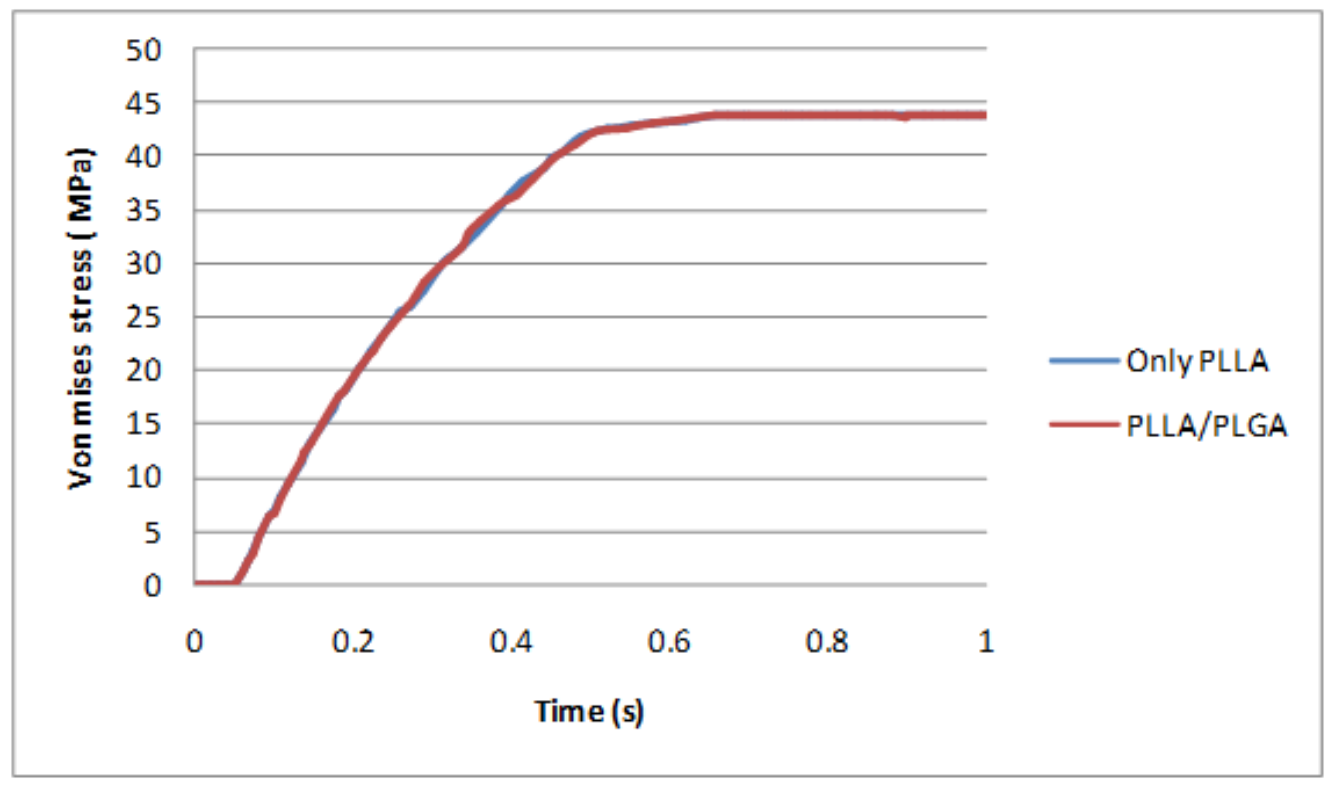

Figure 4-17 von Mises stress changes during expansion of a one-layer PLLA stent and a two-layer PLLA and PLGA stent.

\subsection{Proposed parameters for helical stents and parametric study}

Coils are the building blocks of helical stents. During radial expansion, the coils change in pitch value, uncoil or stretch. On the other hand, expansion of common wire mesh stents includes opening the stent slots and stretching the stent walls. Owing to the differences between these expansion mechanisms, new evaluation parameters were defined in this study to represent helical stent expansion performance.

The change in helical stent length, however, is a cumulative effect based on different factors such as uncoiling, increases in gap length between coils, or an increase in the pitch value of the stent. Thus, two additional parameters, namely uncoiling and change in pitch were introduced to separate these effects.

The number of coils, measured before and after stent expansion, determines stent uncoiling:

Uncoiling $=\frac{\# \text { Coils }_{0}-\# \text { Coils }_{f}}{\# \text { Coils }_{0}} \times 100$

Equation 4-1

where \# Coils ${ }_{0}$ and \# Coils $\mathrm{f}_{\mathrm{f}}$ are the initial and final number of coils, respectively. 
The relationship between foreshortening and uncoiling is semi-independent since the stent pitch will also change during expansion.

The change in the pitch is also relevant:

changeinPitch $=\frac{P_{2}-P_{1}}{P_{2}} \times 100$ Equation 4-2

where $\mathrm{P}_{1}$ and $\mathrm{P}_{2}$ are the stent's pitch values before expansion and after expansion, respectively. This parameter is positive value for increasing pitch corresponding to expanding stent.

\subsubsection{Parametric Study}

Following model development and validation, a parametric study was undertaken to investigate the expansion and recoiling characteristics as a function of the stent geometry (width, thickness, pitch) and coefficient of friction (COF) between the stent and expansion cylinder. For computational efficiency, a 5-coil stent was used. Results from the middle coil were reported since they were representative of 5-coil or longer stents, as described below.

\subsubsection{Number of Stent Coils}

The effect of coil number, ranging from 3 to 8 , on expansion and recoiling was investigated. The stent width $(1 \mathrm{~mm})$ and pitch $(2 \mathrm{~mm})$ were held constant. The evaluation parameters (foreshortening, uncoiling, pitch change and radial recoiling) were relatively constant for stents with 5 or more coils (Figure 4-18). However, for stents with less than 5 coils, a significant increase in pitch and radial recoiling was predicted. This was attributed to the interaction with end coils in these shorter stents, suggesting that stents with less than 5 coils should not be considered for implantation. 


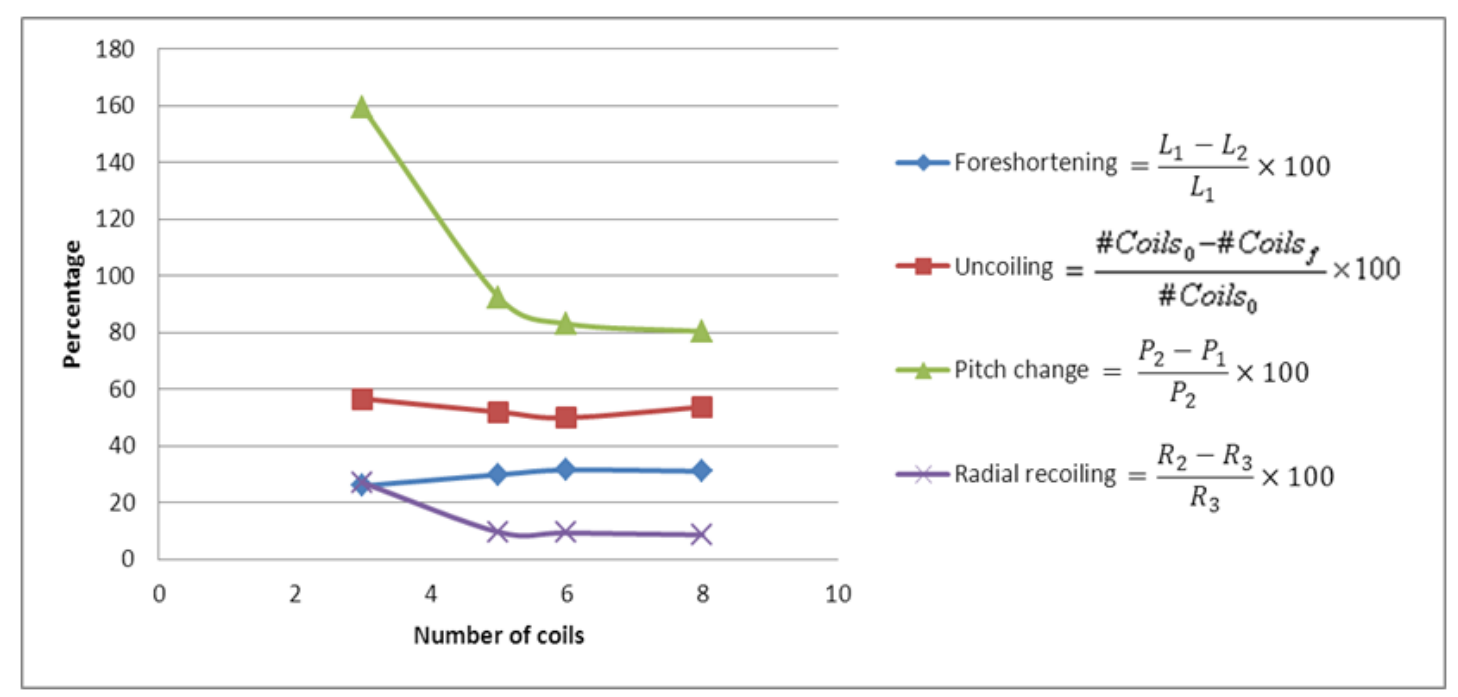

Figure 4-18 Evaluation parameters as a function of number of coil.

\subsubsection{Effect of Friction Coefficient}

Expansion balloons are typically made of polymers such as Nylon, PE, PET, PVC, PTFE and PA, the friction coefficients of which are from approximately 0.04 to 0.2 [Yang, 2010].

Helical stent expansion results in uncoiling as the stent coils slide along the balloon surface. Therefore, in addition to the geometry of the helical stent, the stent-balloon interface can affect both expansion and subsequent recoiling behaviour. These effects were examined in a series of parametric studies on both 5-coil and 8-coil stents with a range of COFs at the stent-cylinder interface. The stent width $(1 \mathrm{~mm})$ and pitch $(2 \mathrm{~mm})$ were held constant. Coil number was varied from 5 to 8 and, since there was no significant change in the results, only the 5-coil results were reported, consistent with other data provided. As expected, increasing the COF resulted in a significant decrease in pitch change and a corresponding increase in foreshortening, while uncoiling and radial recoiling remained constant (Figure 4-19). 


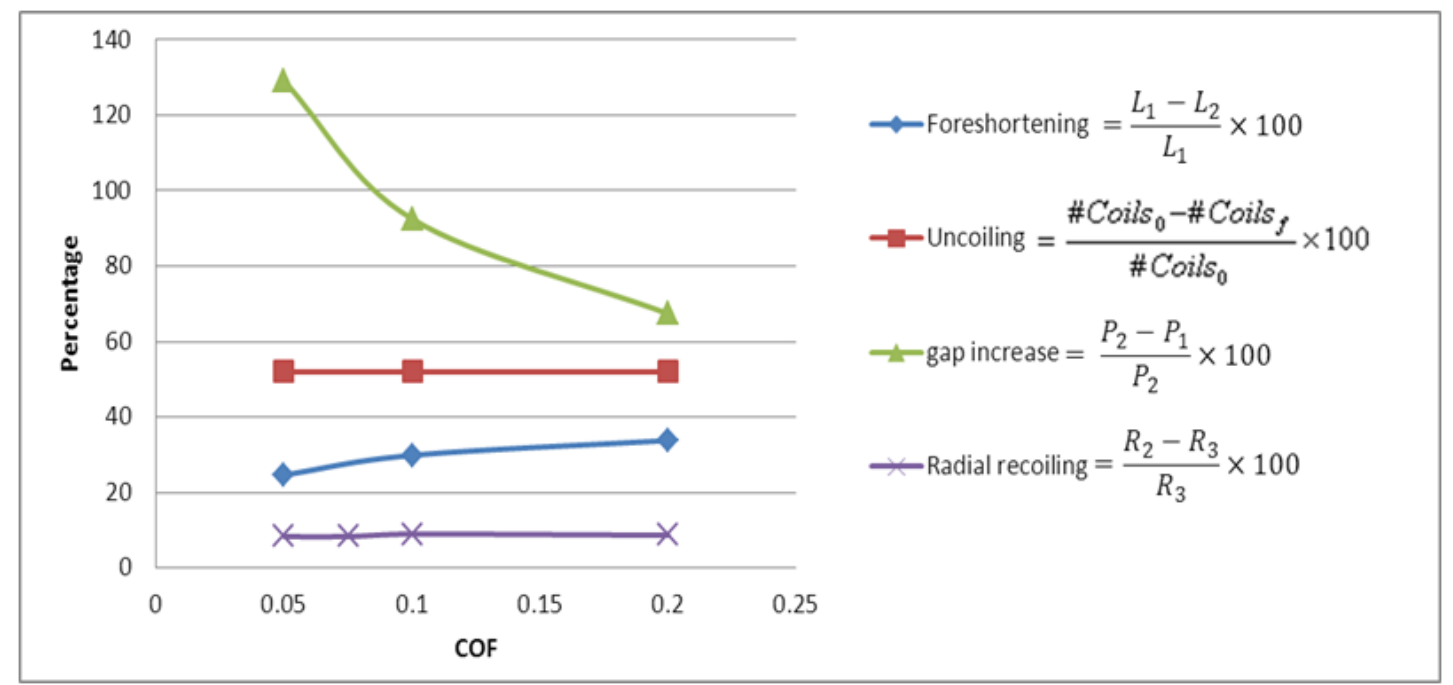

Figure 4-19 Evaluation parameters as a function of COF.

\subsubsection{Stent Width}

The stent width is directly related to the artery coverage area, and is also known to affect drug diffusion patterns from drug eluting stents. The coverage area is important since it is related to the stressed area of the artery during expansion, with larger values being desirable since they result in lower arterial stresses [Rogers, 1999]. A parametric study was undertaken to evaluate a 5-coil helical stent with a $2 \mathrm{~mm}$ pitch and varying widths between $0.5-1.2 \mathrm{~mm}$.

Increasing the stent width (Figure 4-20), increased both the uncoiling and pitch change, and reduced foreshortening due to increased frictional effects. However, radial recoiling (Figure 420) was not significantly affected by the stent width. 


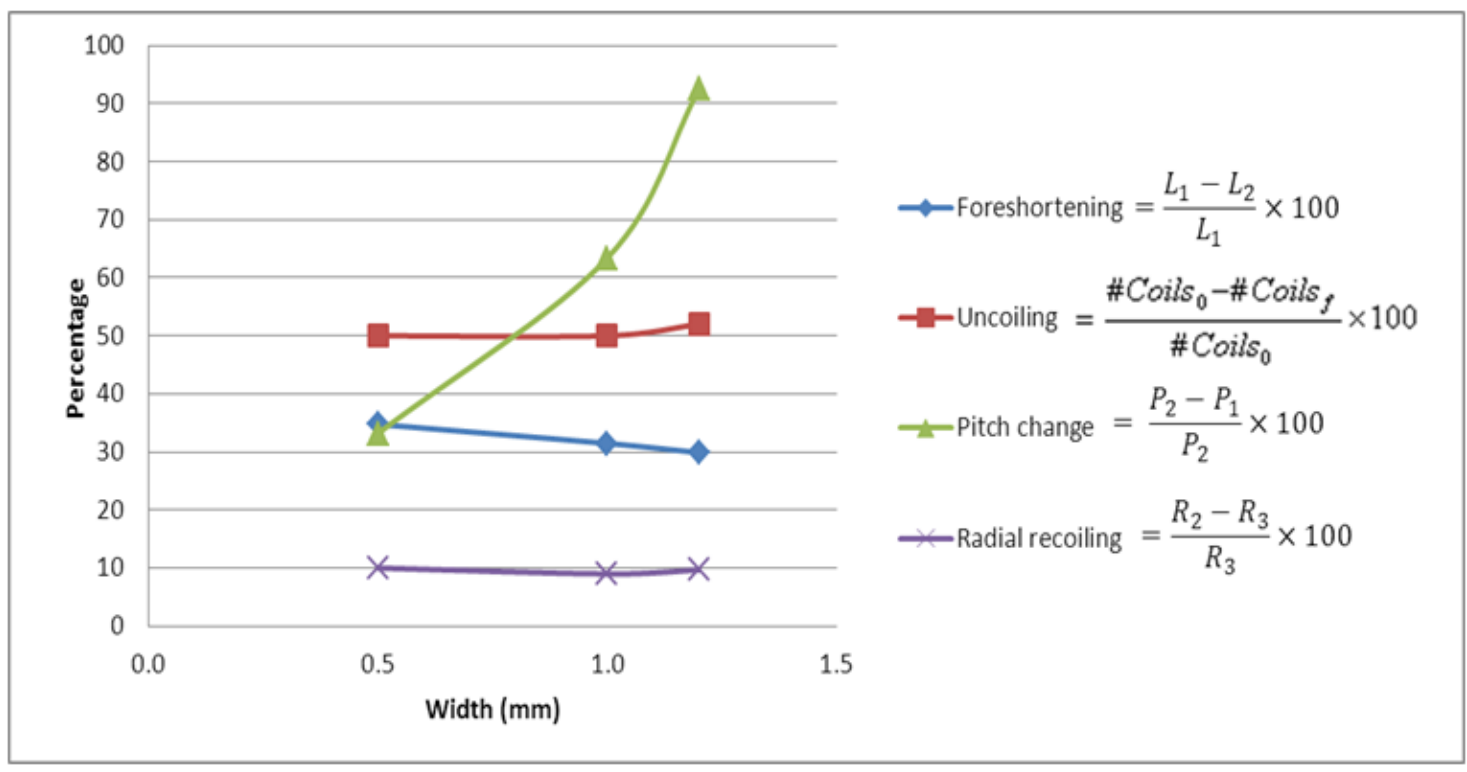

Figure 4-20 Evaluation parameters as a function of stent width.

\subsubsection{Stent Thickness}

Common stent thickness ranges from 0.1 to $0.2 \mathrm{~mm}$. Therefore, in a 2-layer stent with a constant total thickness (here $0.2 \mathrm{~mm}$ ), the share for the PLGA layer can be increased by reducing the thickness of the PLLA layer. Since the PLGA layer is typically the drug-carrying layer, changing its thickness could affect the amount and pattern of drug diffusion. Multiple layers of PLGA can also be included, each containing a different drug. The effect of thicknesses ranging from 0.06 to $0.16 \mathrm{~mm}$ was evaluated using a 5-coil stent with constant width $(1 \mathrm{~mm})$ and pitch (2 mm). The pitch change (Figure 4-21) was predicted to increase with increasing thickness, and produce a corresponding decrease in foreshortening and small decrease in radial recoiling and uncoiling behaviour. In general, thicker stents showed larger plastic deformation, which is ideal for fixing the stent inside the artery. 


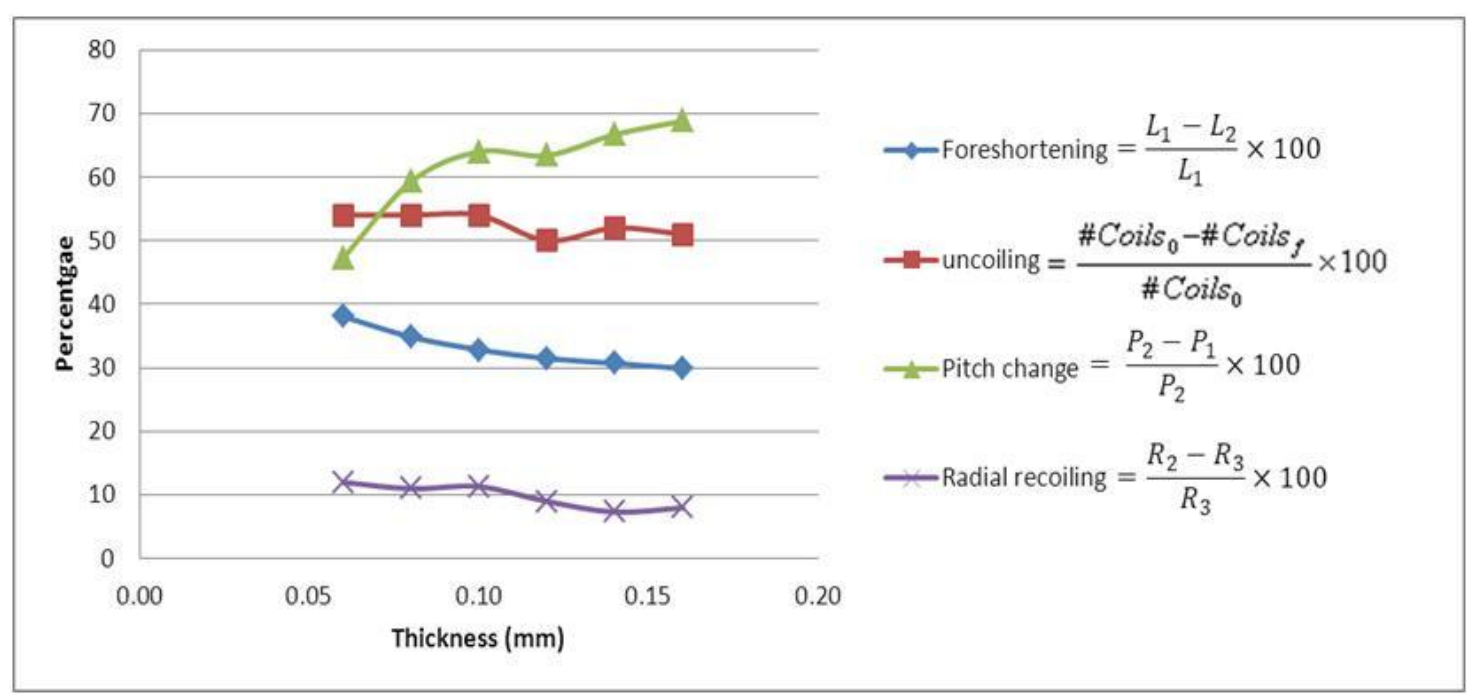

Figure 4-21 Evaluation parameters as a function of stent thickness.

\subsubsection{Stent Pitch}

Stent pitch is relatively easy to modify in the stent manufacturing process, and was investigated for a 5-coil stent with a constant width $(1 \mathrm{~mm})$ and thickness $(0.12 \mathrm{~mm})$. When the pitch value was varied from 1.1 to $2.0 \mathrm{~mm}$, the pitch change decreased significantly (Figure 422). Uncoiling also decreased while foreshortening increased. Radial recoiling, however, remained almost constant with increasing stent pitch. 


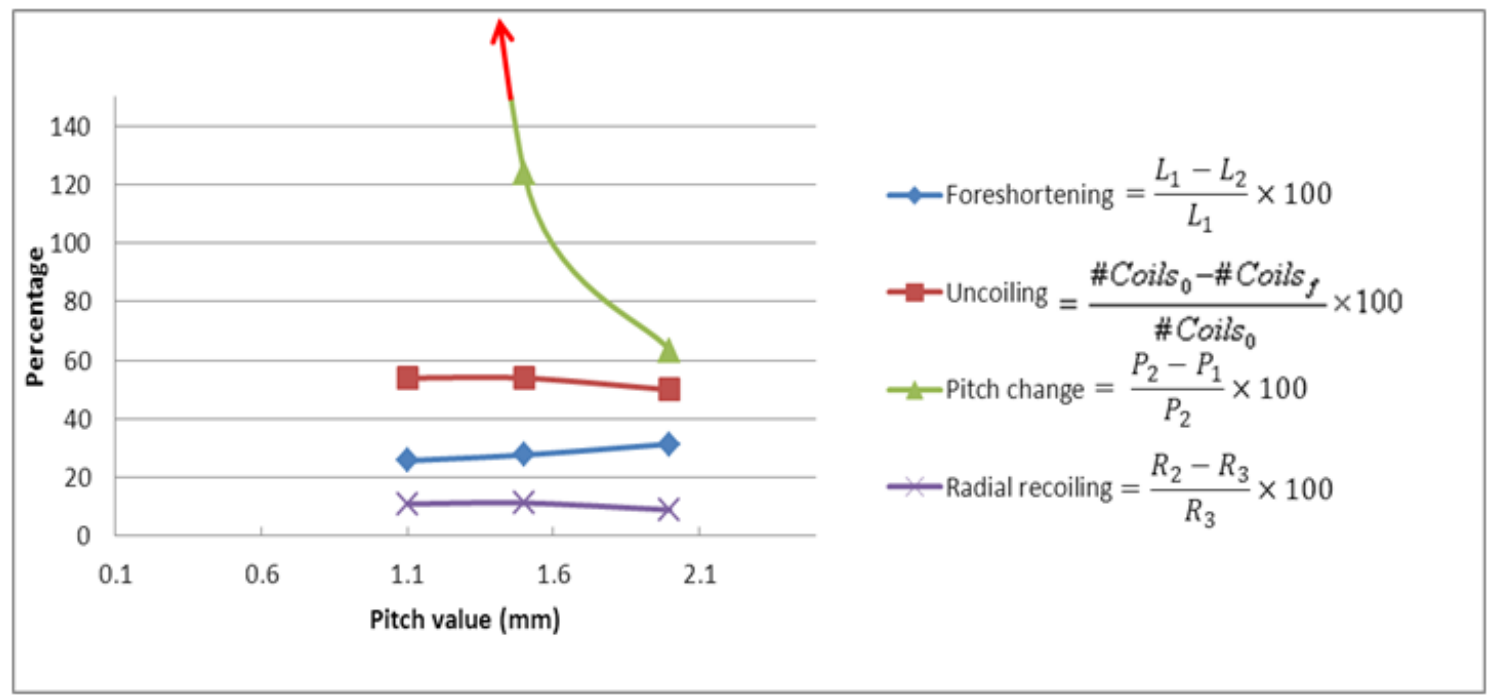

Figure 4-22 Evaluation parameters as a function of stent pitch.

Trends in expansion and recoiling behaviour derived from the parametric study are shown in Table 4-2, where a change in magnitude of more than $5 \%$ was identified as significant.

Table 4-2 Parametric study results for helical stent uniform expansion.

\begin{tabular}{lcccc}
\hline Increased Parameter & Foreshortening & Uncoiling & Pitch change & $\begin{array}{c}\text { Radial } \\
\text { recoiling }\end{array}$ \\
\hline Number of coils & $3-5 \uparrow$ & $3-5 \downarrow$ & $3-5 \downarrow$ & $3-5 \downarrow$ \\
& $5-8 \sim$ & $5-8 \sim$ & $5-8 \sim$ & $5-8 \sim$ \\
COF at the stent- & $\uparrow$ & $\sim$ & $\downarrow$ & $\sim$ \\
cylinder interface & $\downarrow$ & $\uparrow$ & $\uparrow$ & $\sim$ \\
Width & $\downarrow$ & $\downarrow$ & $\uparrow$ & $\sim$ \\
Thickness & $\uparrow$ & $\downarrow$ & $\downarrow$ & $\sim$ \\
Pitch & $\downarrow$ & & \\
\hline
\end{tabular}

$\uparrow:$ increase, $\downarrow$ : decrease, and $\sim$ : constant

The goal of the uniform expansion study was to determine the effect of geometry and friction on expansion and recoiling behaviour. The model was validated based on available experimental 
data. Predicted results were generally within $10 \%$ of the experimental values, where differences were attributed to non-uniform expansion along the stent and interactions with the balloon used in the experiments.

The uniform expansion model results showed that helical stents exhibit a uniform stress distribution after expansion, which is important for controlled degradation when using biodegradable materials. The results indicated that increasing stent width, pitch value and coil thickness resulted in a larger diameter after recoiling, which would improve fixation in the artery. It was also noted that a helical stent should have more than 5 coils to ensure stability after uncoiling.

Helical stent expansion occurs by uncoiling and sliding the coils over the surface of the expander (i.e., the cylinder in this study), where uncoiling primarily generates bending stresses and strains. During expansion, the stent length decreases during uncoiling resulting in longitudinal movement, which is resisted by interface friction and interaction with adjacent coils. These effects can cause a tensile stress in the coils and an increase in pitch (Figure 4-10). The ultimate challenge with these sliding motions in practice (expansion inside an artery) is that they can cause small but significant shear stresses on the delicate artery wall. The evaluation parameters used in this study included traditional measures (such as foreshortening and recoiling) and two proposed parameters (uncoiling and pitch change) to evaluate geometric changes to the stent following expansion and recoiling.

In general, factors that affect the motion of the stent over the expansion cylinder or its bending stiffness will affect the final geometry. Increasing the number of stent coils as well as increasing the friction coefficient at the interface limited stent sliding, which was observed through decreased pitch change (Figure 4-18 and Figure 4-19). Changes in stent length were found to be very sensitive to uncoiling, and the values of foreshortening and pitch change were relatively constant for stents with 5 or more coils. Increasing the stent width increased uncoiling and most significantly affected pitch change (Figure 4-20). Increasing the thickness of stent directly increased the bending stiffness and limited uncoiling (Figure 4-21). As expected, increased thickness resulted in higher plastic strain during expansion and reduced recoiling. Decreasing the pitch value somewhat reduced the stent foreshortening (Figure 4-22). The stent design considered in this study showed $10 \%$ recoiling. It is recommended that the stent should expand up to $10 \%$ of the artery's inner diameter to ensure fixation. 
Based on the above results, polymer helical stent designs exhibit some benefits over traditional materials and stent geometries. Specifically, the helical stent showed a relatively uniform stress distribution, which is favorable for uniform and controlled biodegradation. This also means the amount of permanent deformation and the stent's final geometry can be controlled by the initial stent geometry. In particular, increased thickness was found to increase plastic deformation and therefore fixation in the artery; however, this parameter and the expansion rate must be controlled to avoid excessive strain in the material during deployment. The proposed change in pitch parameter was found to provide a sensitive measure to changes in geometry and should be used for evaluation of proposed helical designs.

Limitations of this model include the need to investigate the effects of transient non-uniform balloon and stent expansion.

The uniform expansion of a 3-coil helical stent was investigated with both implicit and explicit codes. This study revealed that, in a simple uniform helical stent expansion, the results from of implicit and explicit codes are identical. The explicit code, however, takes a long time to run and requires scaling. Thus, different scaling levels were applied on the model; a comparison of the produced energy error and resultant mechanical outputs showed that just observing the error levels is not sufficient to capture the effects of contact with mass and time scaling. Therefore, to avoid inaccurate results, implicit modeling should be used whenever possible. Explicit code is essential to solving models that include contact interfaces and nonlinearities. Experimental verification and validation should also be undertaken in these cases.

\subsection{Stent- folded balloon expansion}

\subsubsection{Developing tri-folded balloon geometry}

The balloon model was first created using the geometry of a fully expanded balloon $(3 \mathrm{~mm}$ diameter, $0.03 \mathrm{~mm}$ wall thickness) [Bravo] with a small internal pressure (200 Pa) to maintain stability. Due to symmetry, only one half of the balloon was modeled, with a length of $3.5 \mathrm{~mm}$ and meshed with 37200 shell elements using the Belytschko-Tsay formulation [Belytschko, 1984]. Three integration points were used through the balloon thickness to minimize element distortion during folding and wrapping. Early investigations showed that the presence of a central wire $(\mathrm{CW})$ did not affect the stent expansion results; thus, in order to minimize 
computational time, a central wire was not included in the present expansion model. The end nodes of the balloon were fixed in the radial, circumferential and axial directions to accommodate removal of the central wire. It was found that a fine mesh (approximately 0.03 $\mathrm{mm}$ elements) was required to prevent significant element distortion during balloon folding and wrapping. Any excessive distortion in the folding phase produced asymmetric balloon expansion. The balloon was folded by introducing rigid surfaces (Figure 4-23), which is similar to an actual balloon folding process. It was found that sharp corners were necessary to produce folds that could wrap around the central wire. In addition, a slight negative pressure was included during the folding phase to maintain numerical stability of the model.

The balloon material properties (nylon-12) were defined as an isotropic elastic material with a modulus of $0.5 \mathrm{GPa}$ [Matweb, 2012] and Poisson's ratio of 0.3 [Oberhofer, 2006]. After completing the folding simulation, the folded half balloon was mirrored and the interface nodes were merged together to create the full balloon (124080 shell elements, $13.8 \mathrm{~mm}$ length). Before merging, the element normal vectors were set to be in the same direction to ensure numerically stable contact during the expansion simulation. The body size of the balloon (the length of the balloon between the two end caps) was equal to the length of the unexpanded stent. 

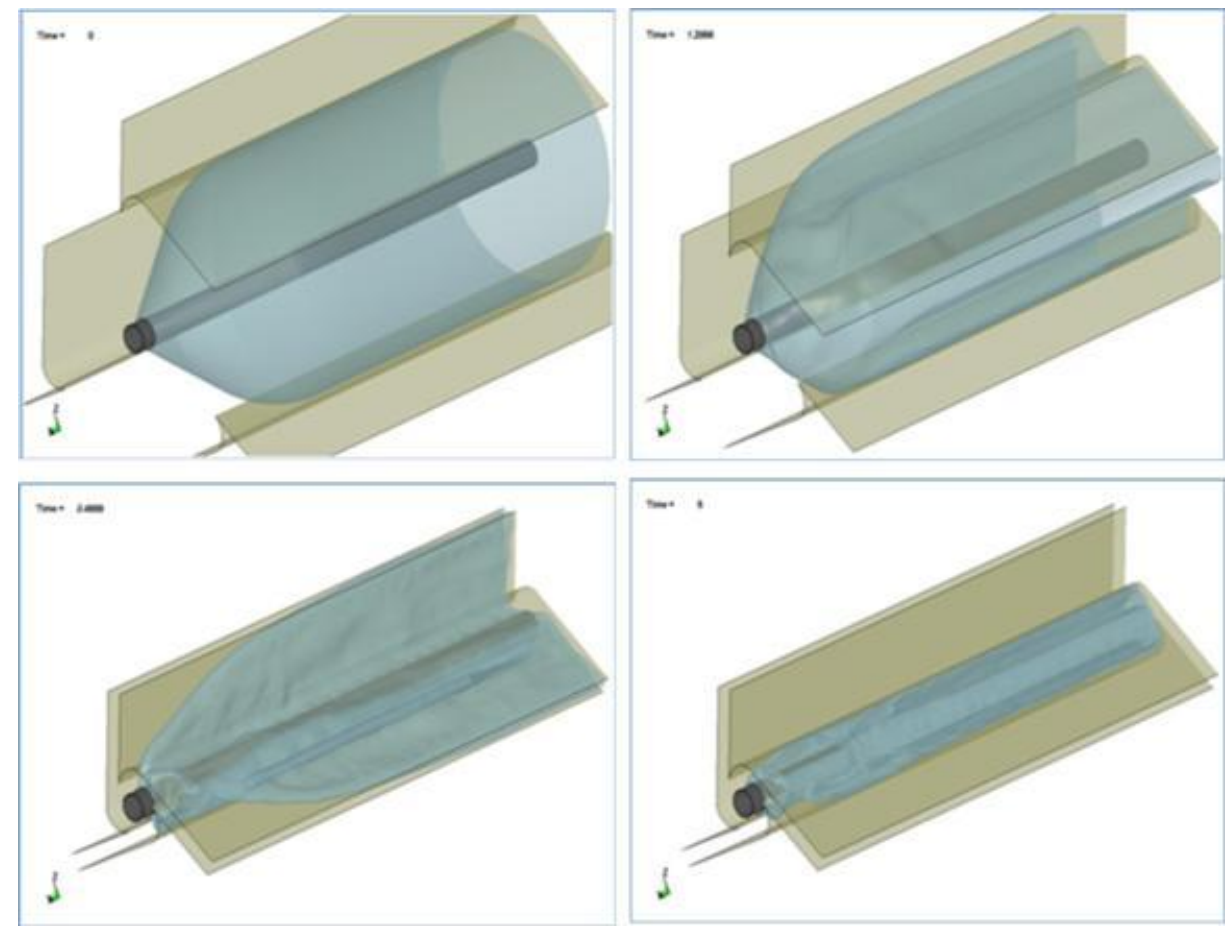

Figure 4-23 FE model of the balloon folding process (3-fold).

To verify the folded balloon model, the balloon was expanded using internal pressure and the pressure-diameter results of the expansion model were compared to the results provided by the balloon manufacturer [Bravo] (Figure 4-24). Diameter measurements taken before reaching the expanded condition (less than 2.8 bar) was somewhat subjective and the difference between the FEM and manufacturer data may not have any physical representation. The maximum pressure is 12 bar, which is the balloon's rupture pressure, whereas the nominal pressure of this balloon is 8 bar. This means that the balloon will reach its nominal diameter (without a stent) at 8 bar. 


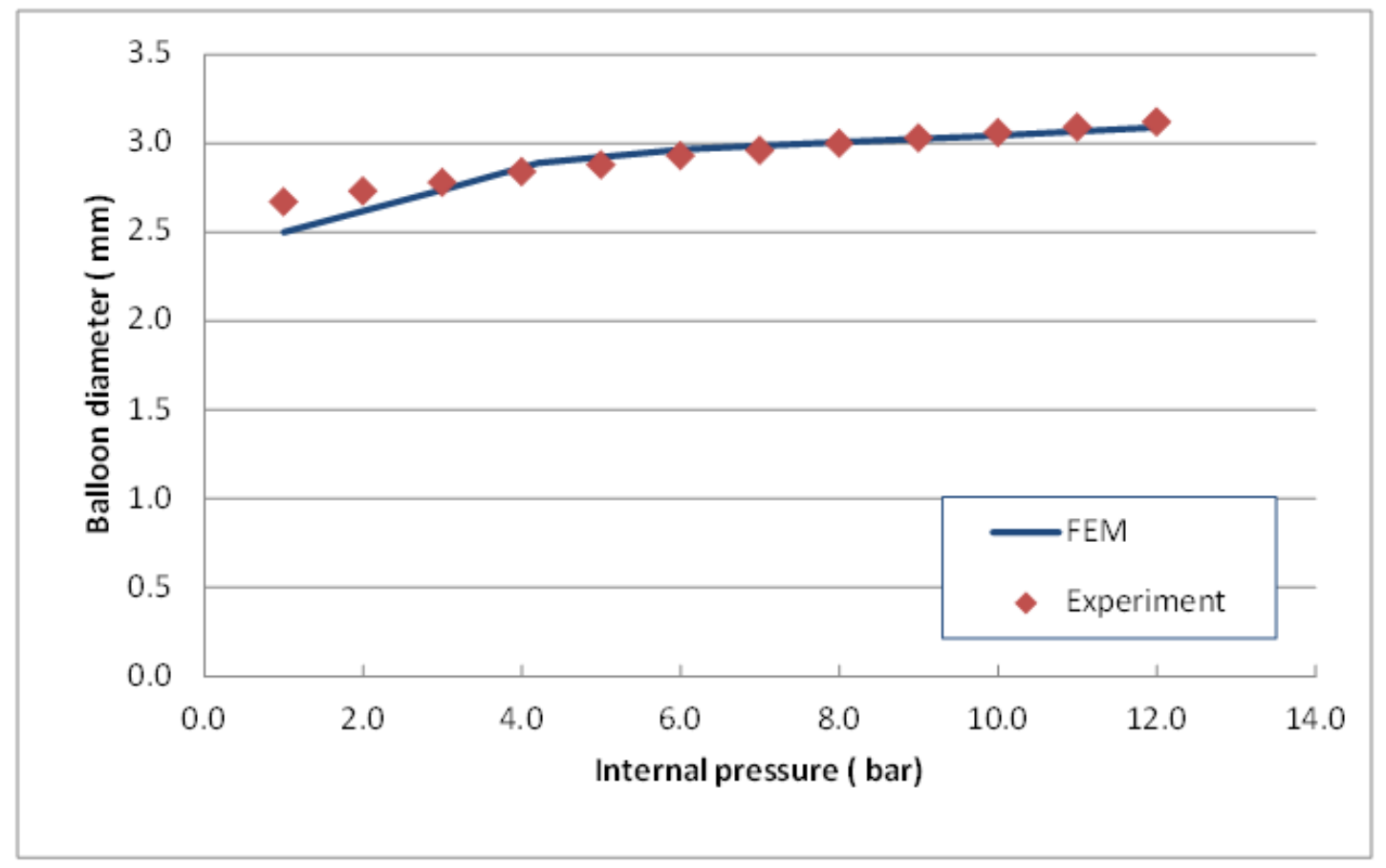

Figure 4-24 Verification of free expansion of a folded balloon.

\subsubsection{Stent Expansion using a folded balloon}

A finite element model of a helical PLLA stent ( 5 coils, $1 \mathrm{~mm}$ width, $2 \mathrm{~mm}$ pitch, and 0.12 mm thickness) was developed, as discussed in Section 4.4. The stent was meshed with 6600 hexahedral elements and the mechanical properties of the PLLA were measured in vitro $\left(37^{\circ} \mathrm{C}\right.$ water) using an immersed dynamic mechanical analyzer (DMA). The stent was implemented in the model using an elastic-plastic constitutive model with Von Mises yield criterion and an elastic modulus of $1.4 \mathrm{GPa}$, Poisson's ratio of 0.3 , and yield stress of $260 \mathrm{MPa}$.

The helical stent model was coupled to the folded balloon model (Figure 4-25) using a contact algorithm with a COF of 0.1 at the balloon-stent interface [ $\mathrm{Vad}, 2010]$ while frictionless selfcontact was applied between the balloon folds [Oberhofer, 2006]. A self-contact was also applied between the stent coils to capture any interaction between the coils during expansion and a monotonically increasing pressure was applied to the inner surface of the balloon to a maximum of 8 bars, based on the manufacturer's specifications. 


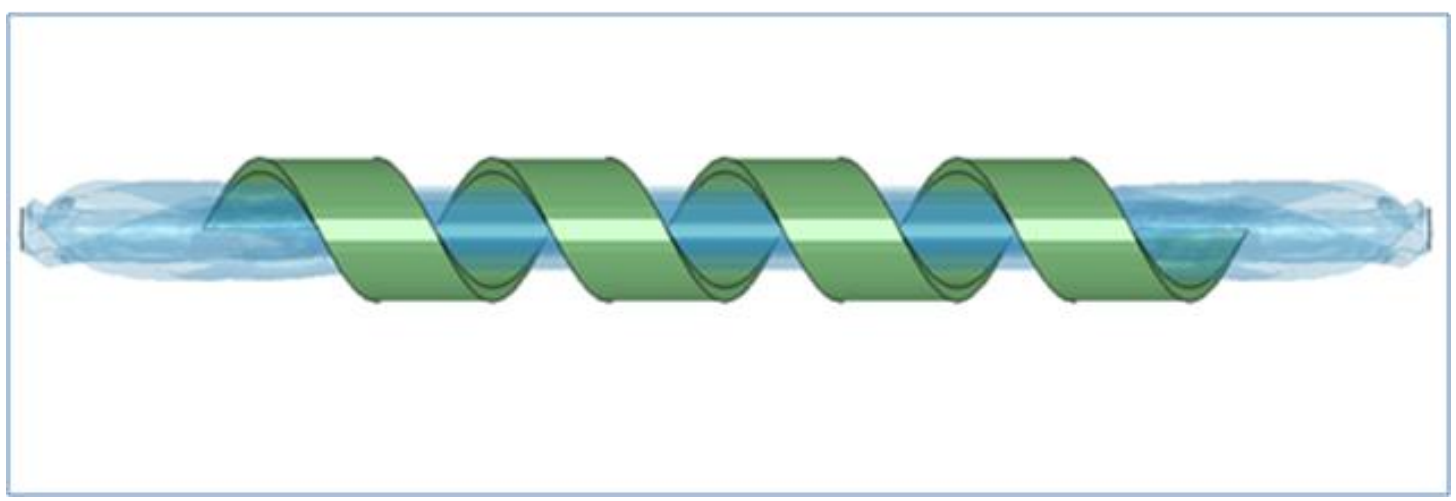

Figure 4-25 FE model of a coupled stent and tri-folded balloon.

The model was solved using an explicit finite element code (LS-Dyna, LSTC) since an implicit approach was found to be unstable for the complicated contact between the balloon and stent. Both time and selective mass scaling were used for the folding and expansion phases, and the component inertia and kinetic energy were monitored and found to be less than $5 \%$ of the total energy, which was considered acceptable for this type of simulation. The model was solved on an SMP platform (AMD X86_64 GNU/Linux with 8 CPUs $(4 * 2.11 \mathrm{GHz})$ ), which typically took 56 minutes to fold the balloon and 48 hours to expand the coupled stent-balloon model.

Following model development, a convergence study was conducted to determine the mesh resolution required for the stent. To allow for reasonable computation times, a representative three coil stent was expanded using the tri-folded balloon and the level of equivalent plastic strain was monitored for different element densities (Figure 4-26). The same element density $(0.03 \mathrm{~mm})$ was used for the full model, corresponding to 4 elements through the thickness of the stent. 


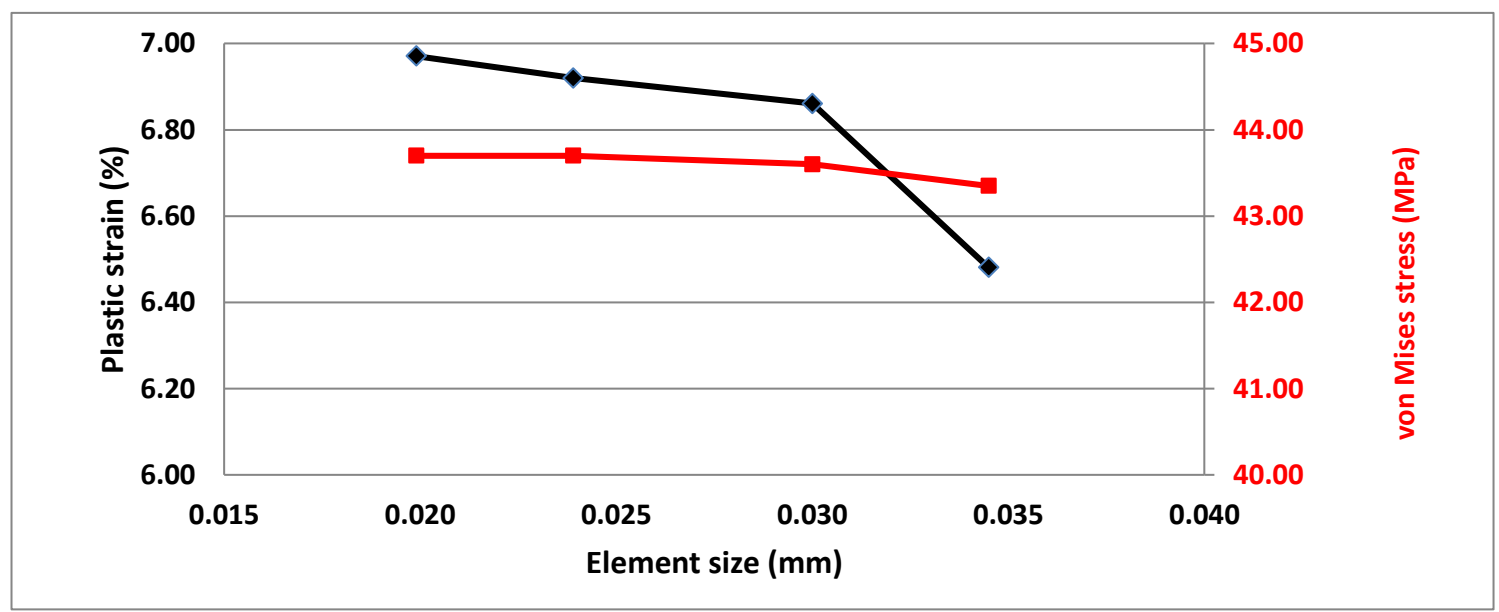

Figure 4-26 Convergence study on the element size of the stent.

\subsubsection{Checking the energy balance}

As the stent balloon expansion is very time consuming, in addition to parallel computing time and selective mass scaling was used to decrease the run time. To ensure that the inertia effect did not decrease the accuracy of the results, the ratio of kinetic to total energy was monitored and found not to be less than $5 \%$ (Figure 4-27). 


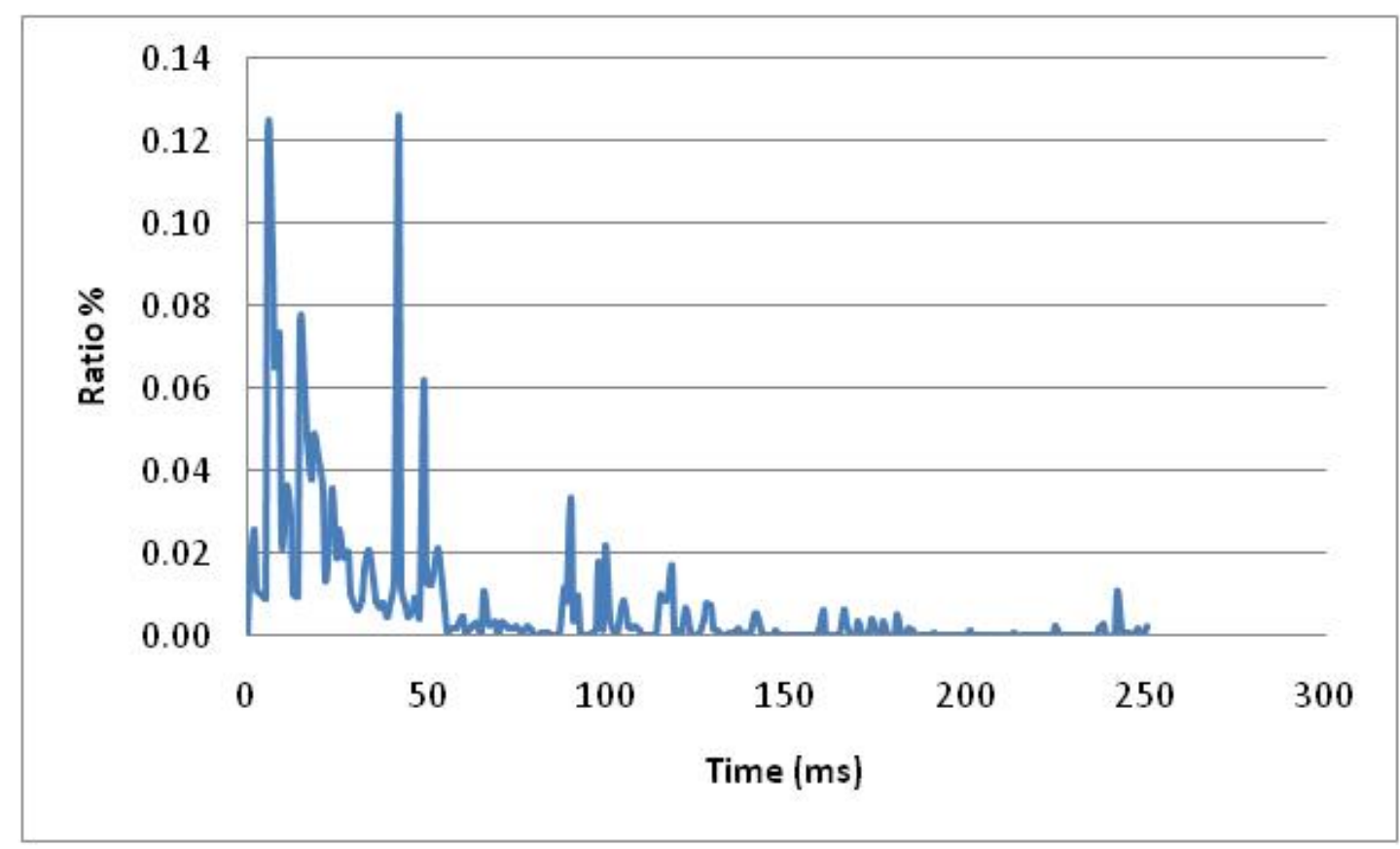

Figure 4-27 A typical kinetic-to-total energy ratio model of helical stent expansion with folded balloon inflation (using LS Dyna).

\subsubsection{Typical balloon expansion of a helical stent}

The third expansion method (folded balloon inflation) was investigated using a folded balloon model (Figure 4-28) to accurately represent the actual expansion conditions. This method was computationally expensive due to the complicated contact conditions, where the initial expansion phase resulted in significant non-uniform deformation (dog boning) at the ends of the stent, causing the coils to slide axially over the balloon and interact. This was in agreement with experimental tests (Figure 2-2). The expansion process for the stent geometry was terminated prior to full expansion due to the excessive element distortion resulting from interaction between and overlap of the coils (Figure 4-28). During expansion, the balloon interface provided some axial motion restriction through friction; however, the local deformation tended to overcome frictional effects causing contraction in the middle of the stent, which was enhanced by the low axial stiffness of the helical stent. 

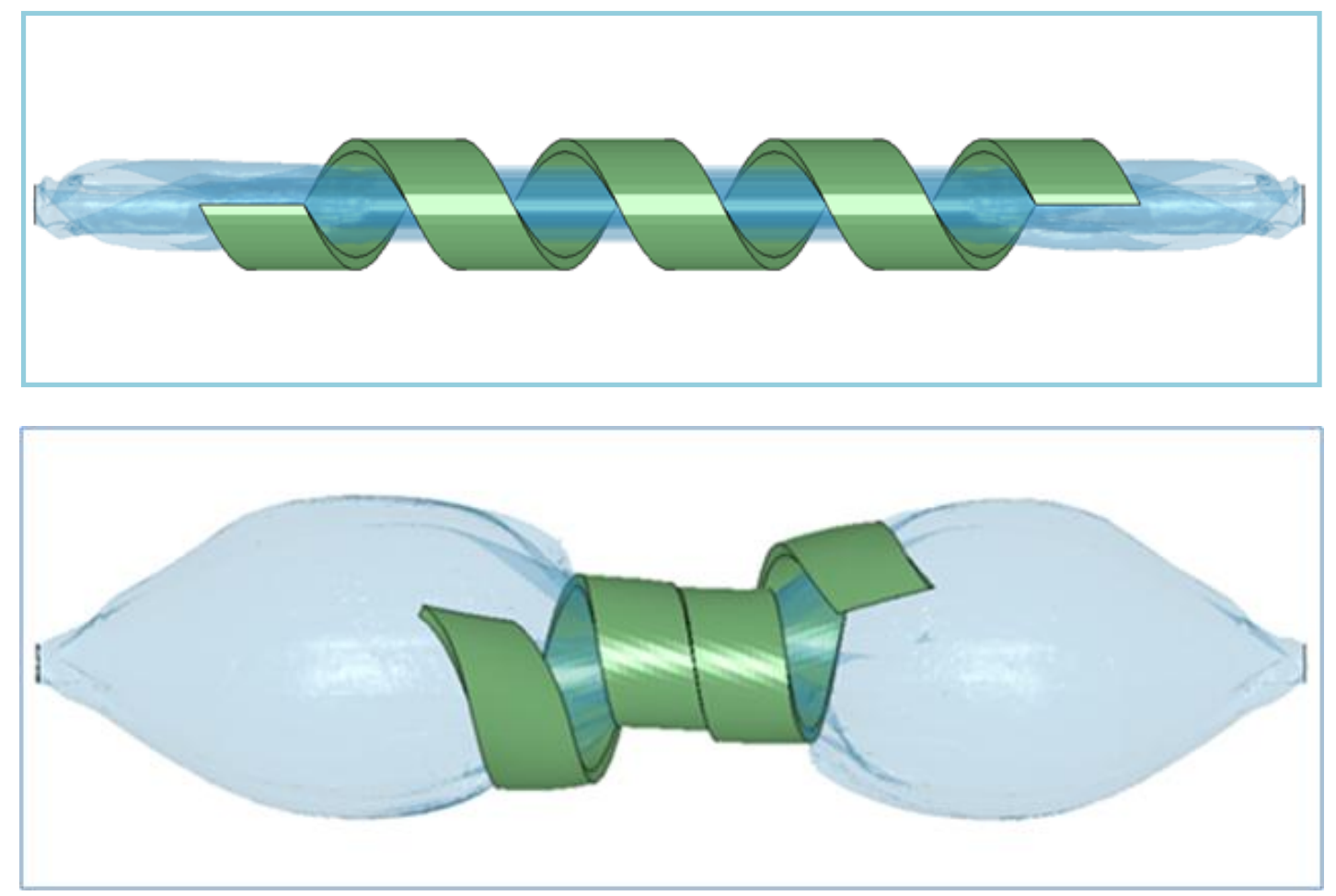

Figure 4-28 Typical helical stent expansion with a folded balloon (1.42 mm expansion).

The helical stents were expanded from the inner diameter of $1 \mathrm{~mm}$ to $3 \mathrm{~mm}$. Folded balloon expansion is the most realistic method to expand a stent. This method shows the helical stent's sensitivity to dog boning. The whole expansion process was terminated due to overlapping of the coils, which is promoted by dog boning. During expansion, the balloon provides some axial restrictions through friction. However, the dog boning seen in both the balloon and stent causes the stent to overcome the frictional effect and shrink in the middle. As the stent structure does not have any axial restriction, there is no other means to prevent the coils from overlapping, which halts expansion. Combined, the helical stent's sensitivity to dog boning and the inherent tendency of balloons to expand in a dog boning shape prevent the stent from expanding properly with commercially available balloons.

The comparison of different numerical expansion methods showed that the model that describes dog boning in helical stents couldn't be investigated with simplified expansion methods (e.g., uniform expansion). Based on the results of this study it is evident that numerical 
methods can only capture realistic helical stent expansion when folded balloon inflation is considered.

This study determined that balloon expansion is the only realistic method to predict stent expansion, and identified non-uniform expansion (dog boning) as an important issue to address for proper helical stent deployment.

Using the folded balloon expansion method, it was evident that available commercial balloons promote dog boning and overlapping in helical stents. Therefore, using a new designed balloon that can expand the stent progressively from inside out is essential for deployment of this type stent.

The folded balloon expansion method was found to be the most realistic expansion method, albeit with significant computational cost relative to the other models (approximately 32 times longer compared to the uniform expansion model).

\subsubsection{Unsymmetrical expansion of helical stent with folded balloon inflation}

The expansion of the stent usually starts from two ends. Radial expansion at two ends, however, is not equal at the same pressure level. This is due to the fact that the stent coils and the balloon are both folded. During expansion, both the balloon and stent folds open (Figure 429). During expansion, the balloon unfolds in a predetermined direction. When that direction corresponds to the uncoiling direction of the stent, they expand together normally. However, at the other end of the stent, the balloon unfolds and the stent uncoils in opposite directions. At this end, expansion is higher at any given pressure than at the other end of the stent-balloon. This causes unsymmetrical expansion at ends of the stent, which is different from the dog boning effect. 

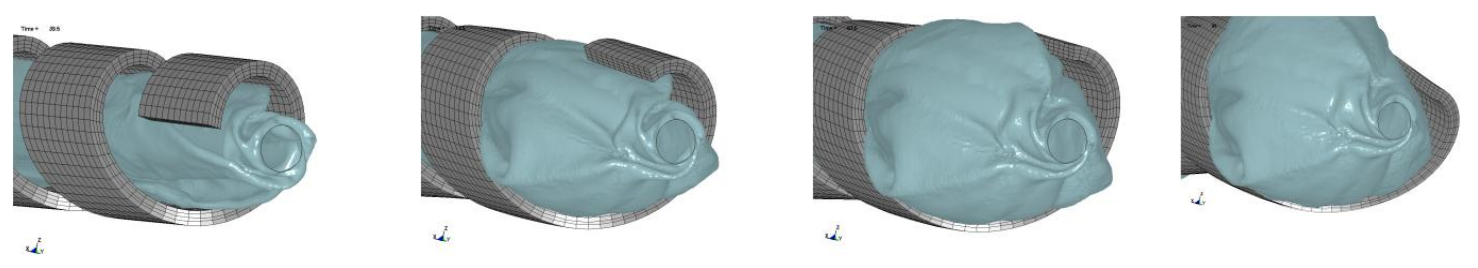

Figure 4-29 Unsymmetrical expansion of a helical stent. The end shown here expands faster than the other because the preferred direction of the balloon is in the opposite direction of the stent folds.

\subsection{Expansion mechanism in a helical stent}

Based on both modeling and experimental results, it seems helical stent expansion occurs via three concurrent deformation mechanisms:

1. Decrease in the pitch value of the helix.

This effect can be seen in Figure 2-2 (middle) where the expansion initiates at the ends of the stent and the coil pitch becomes smaller in the expanded coils (Figure 4-30).

2. Stretch in the coils of the helix.

In the expanded part of the stent (Figure 2-2), the coils become narrower allowing for some increase in diameter (Figure 4-30). This is generally considered a secondary effect in terms of magnitude and is dependent on friction between the balloon and stent.

3. Uncoiling of the helix.

As the stent expands, coil number decreases due to uncoiling (Figure 2-2) and (Figure 4-30).

The geometry of the stent (e.g., width and number of coils) as well as the boundary conditions on the stent (e.g., COF at the balloon and stent interface) can change the contribution of each mechanism in the radial expansion of the stent [Paryab, 2012]. 


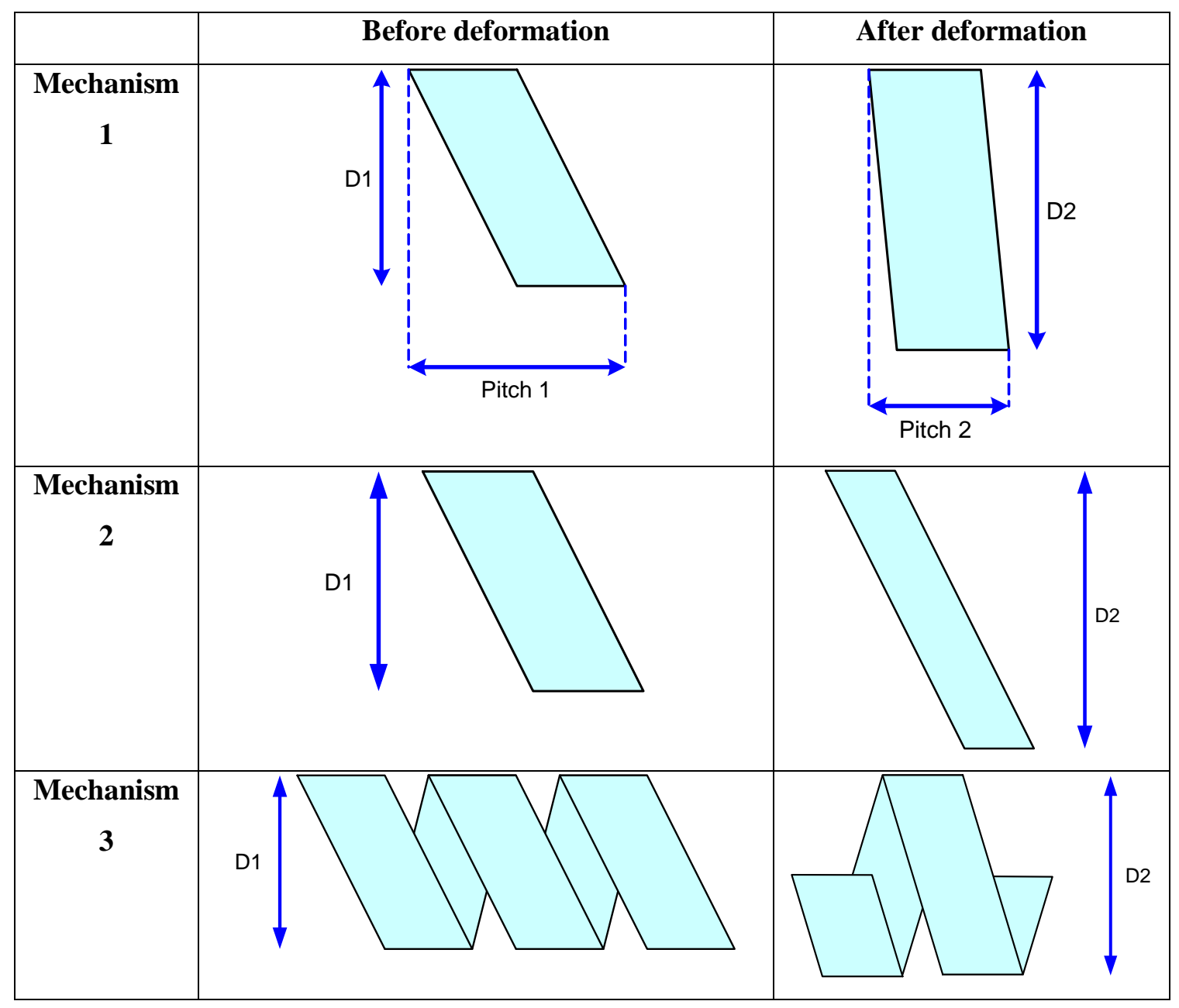

Figure 4-30 Helical stent expansion and deformation mechanisms. 


\section{Chapter 5 \\ Challenges to achieving full expansion of helical stent}

\subsection{Introduction}

Non-uniform expansion is a major issue for helical stent design, with dog boning and coil overlap, both shown in Figure 2-2, posing the primary challenges to clinical deployment. This chapter describes a series of studies undertaken to investigate the effect of various factors on stent expansion. Factors include those related directly to stent design (width, thickness, pitch value, and coil number), those related to the balloon deployed to expand the stent (length, cap length, wall stiffness, and wall thickness), and the coefficient of friction (COF) at the balloonstent interface. The chapter concludes with several recommendations to reduce and solve both dog boning and coil overlap.

Section 5.1 summarizes the abovementioned expansion studies, placing particular emphasis on those factors that potentially lead to dog boning and coil overlap in an effort to identify an effective method to minimize these issues and support proper stent expansion. Each of these studies is based on finite element results using explicit FE code (LS Dyna). In each case, a folded balloon (tri-folded, unless stated otherwise) was used to expand the stent and the element density of the stent was defined based on the converged value reported in Chapter 4.

During helical stent expansion, foreshortening starts from the end coils and is triggered by dog boning in the stent-balloon system. From studies that investigated other balloon-expandable stent types it is known that the relative length between the stent and balloon plays an important role in producing the dog boning effect [Kiousis, 2009]. Therefore, in the parametric studies discussed here, the relative stent-balloon length is held constant. In cases where the width of the stent's pitch value was changed, which then affects the length of the stent itself, stents with higher coil counts were used to correct the stent length.

Since stent expansion terminates as the end coils begin to overlap (Figure 2-2), models were terminated at the first observed sign of overlap.

\subsubsection{Effect of coil number}

Coils are the building blocks of helical stent design. During expansion, the helical stent uncoils and as each coil moves there is a risk of overlap, particularly in the end coils. The 
number of coils in the stent plays an important role in this behaviour; therefore, the effect of coil counts on dog boning and coil overlap was examined in this study.

A tri-folded balloon was inflated to expand PLLA stents with the following dimensions: $1 \mathrm{~mm}$ width, $2 \mathrm{~mm}$ pitch value, $0.12 \mathrm{~mm}$ thickness, and 0.1 COF at the balloon-stent interface. Each stent had one coil (Figure 5-1), two coils (Figure 5-2), three coils (Figure 5-3) or 4 coils (Figure $5-4)$.

One-coil stent expansion is independent of the effect of any other attached coil and is dominated by the uncoiling (bending) of a curved structure. This deformation was well predicted by the theory of curved membrane bending in the small strain range.

The folded balloon was expanded from a very small diameter to a full cylinder with an inner diameter of $3 \mathrm{~mm}$ (except for the end caps which have smaller final diameters). Full expansion of the one-coil model was successful, and the stent followed the balloon expansion to its full nominal diameter. During expansion, the coil expanded to half a coil in the nominal expanded diameter (uncoiling factor is about 50\%) (Figure 5-1). Therefore, the final geometry of the expanded one-coil stent cannot cover the full cylinder. This suggests that without benefit of neighboring coils, uncoiling behaviour paired with a decrease in pitch value causes one-coil helical stents to expand.

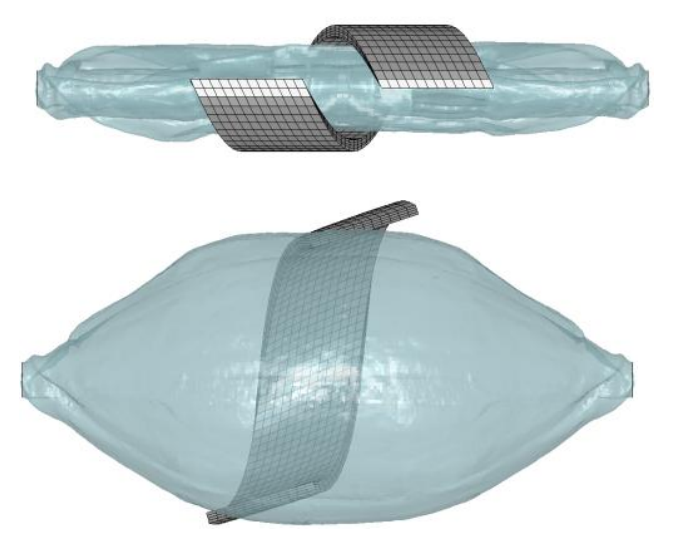

\section{Figure 5-1 One-coil helical stent expansion.}

By adding one coil to the expansion system, both coils are restricted at one end. Therefore, it is easier for each coil to expand from the free end. This type of expansion is caused by uncoiling 
and is dominated by bending (deformation) of a curved membrane. However, the deformation of each coil is independent from the neighboring coil. Expansion was successful, and the two-coil stent followed the balloon expansion to the full nominal diameter (Figure 5-2). In this case, the uncoiling factor is $60 \%$ and the number of coils after expansion is less than one.

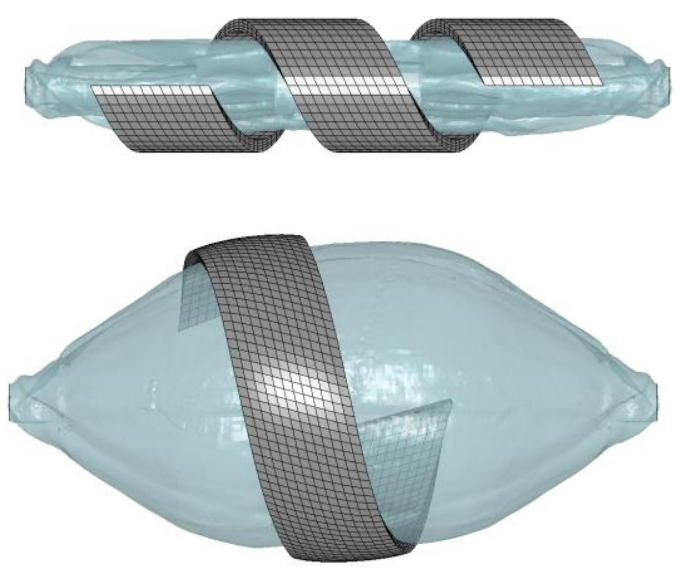

\section{Figure 5-2 Two-coil helical stent expansion.}

During three-coil stent deployment, two end coils and one middle coil expand. Expansion of the middle coil is caused expansion of the coil itself and also the uncoiling of the two ending coils; that is, as the stent expands, the end coils unfurl, the middle coil stretches and the pitch of the stent changes. However, these helical stents demonstrate asymmetrical expansion phenomena, where one of the end coils expands at a faster rate than the other. This causes the middle coil to slip distally towards the slower expanding end. Further expansion of the balloon pushes the stent off the folded balloon completely, and expansion ceases. (Figure 5-3). Asymmetrical expansion is a critical shortcoming of the three-coil stent geometry, and suggests helical stents should have at least four coils for practical implementation. This adds sufficient length to the stent, ensuring that asymmetrical expansion at one end does not affect expansion at the other. 

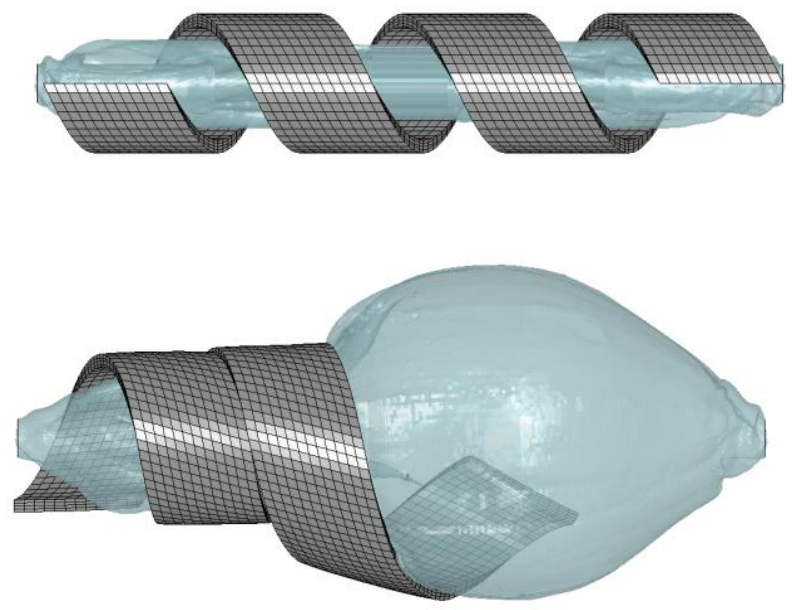

Figure 5-3 Three-coil helical stent expansion.

During four-coil stent expansion (assuming the same pitch, coil width and depth as the earlier described three-coil stent), expansion of the two middle coils depends on the expansion of the end coils. During the first phase of expansion, one end of the stent expands at a faster rate than the other. However, the frictional resistance force between the stent and balloon does not allow the stent to slide off the balloon.

Dog boning occurs at both ends of the stent, which causes the stent ends to expand more rapidly than the middle coils can unravel. Expansion of the two end coils (i.e., dog boning) pushes the stent to the middle of the balloon (Figure 5-4). The middle coils then overlap, preventing full expansion of the stent. The model was terminated by the user upon first observation of coil overlap. 

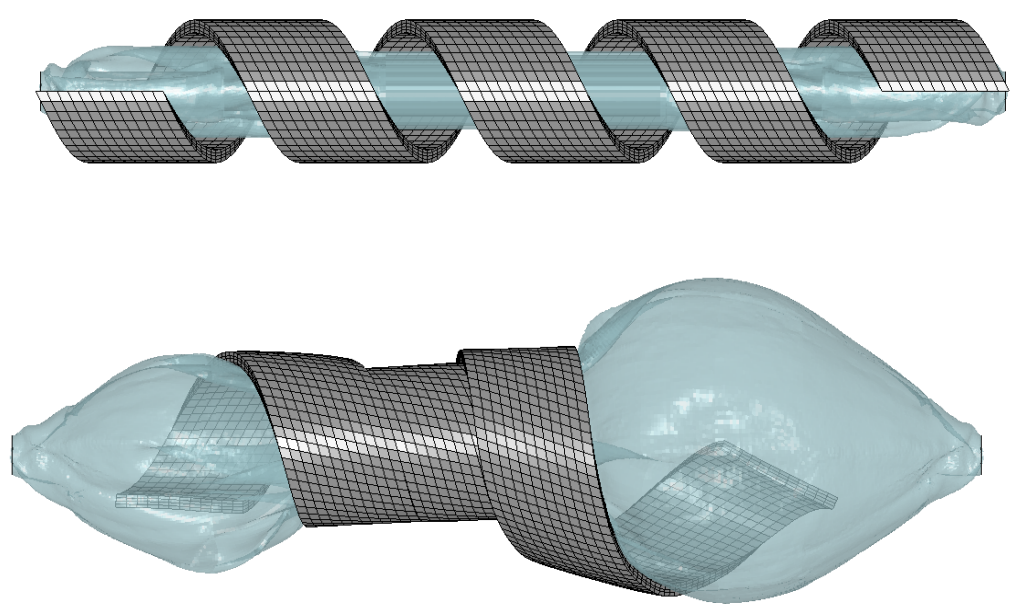

Figure 5-4 Four-coil helical stent expansion.

The parametric study undertaken to elucidate the effect of coil number on stent expansion revealed two important considerations for helical stent design: i) helical stents should have at least four coils to overcome the effect of asymmetrical expansion, where the expansion of one end did not affect the expansion at the other end; and, ii) dog boning causes the coils to overlap in the middle of the balloon, preventing full stent expansion.

\subsubsection{Effect of stent width}

To fully understand the effect of coil width on stent expansion, a parametric study was undertaken that varied stent width, starting with the narrowest coils possible, while holding stent depth and pitch value constant. Stent thickness is another geometrical factor that can affect its expansion. Decreasing the coil thickness not only reduces the bending strength of the structure, and thus facilitates uncoiling behaviour, but also reduces the friction force (by reducing the normal acting force) at the stent-balloon interface. Therefore, a smaller width stent should demonstrate improved uncoiling. On the other hand, radial strength is a crucial design characteristic; stents must have sufficient radial strength both during and after deployment to withstand pressure from the surrounding artery and possibly any residual plaque buildup to prevent artery closure.

The four-coil stents studied here each had a thickness of $0.12 \mathrm{~mm}$, pitch of $2 \mathrm{~mm}$, and 0.1 $\mathrm{COF}$ at the stent-balloon interface, while the stent widths were varied: $0.12 \mathrm{~mm}$ (Figure 5-5), $0.24 \mathrm{~mm}$ (Figure 5-6), and $0.36 \mathrm{~mm}$ (Figure 5-7). 
The $0.12 \mathrm{~mm}$ width stent had a square cross-section and showed low radial strength. While this stent expanded successfully, the end coils demonstrated undesirable rotation (Figure 5-5).
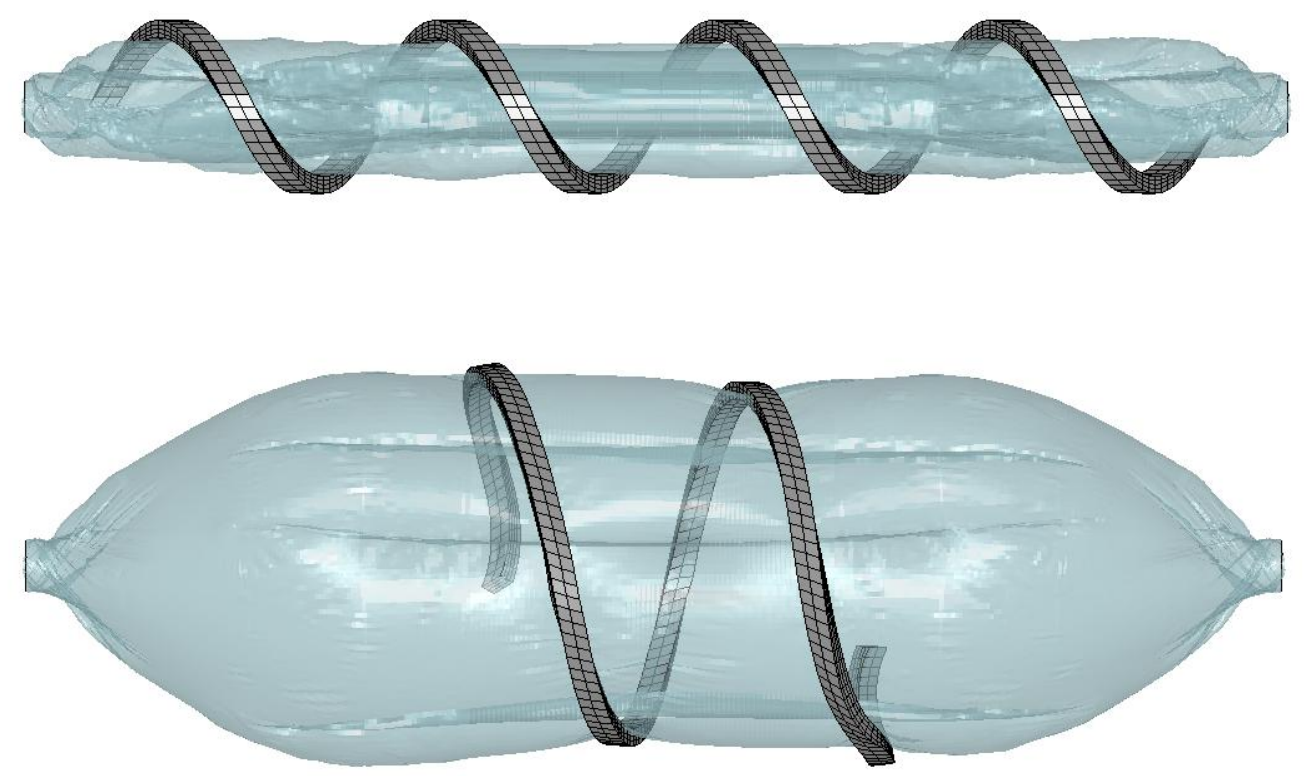

Figure 5-5 Expansion of a $0.12 \mathrm{~mm}$ (width) four-coil stent.

By increasing the width of the helical stent to $0.24 \mathrm{~mm}$, both the bending strength and frictional force at the stent-balloon interface increased. Both factors slow uncoiling behaviour and enhance the effects of other expansion mechanisms (e.g., stretching of the coils). While stent expansion was successful, the end-coils showed similar torsional rotation to the $0.12 \mathrm{~mm}$ stent (Figure 5-6). No dog boning was observed during expansion. 

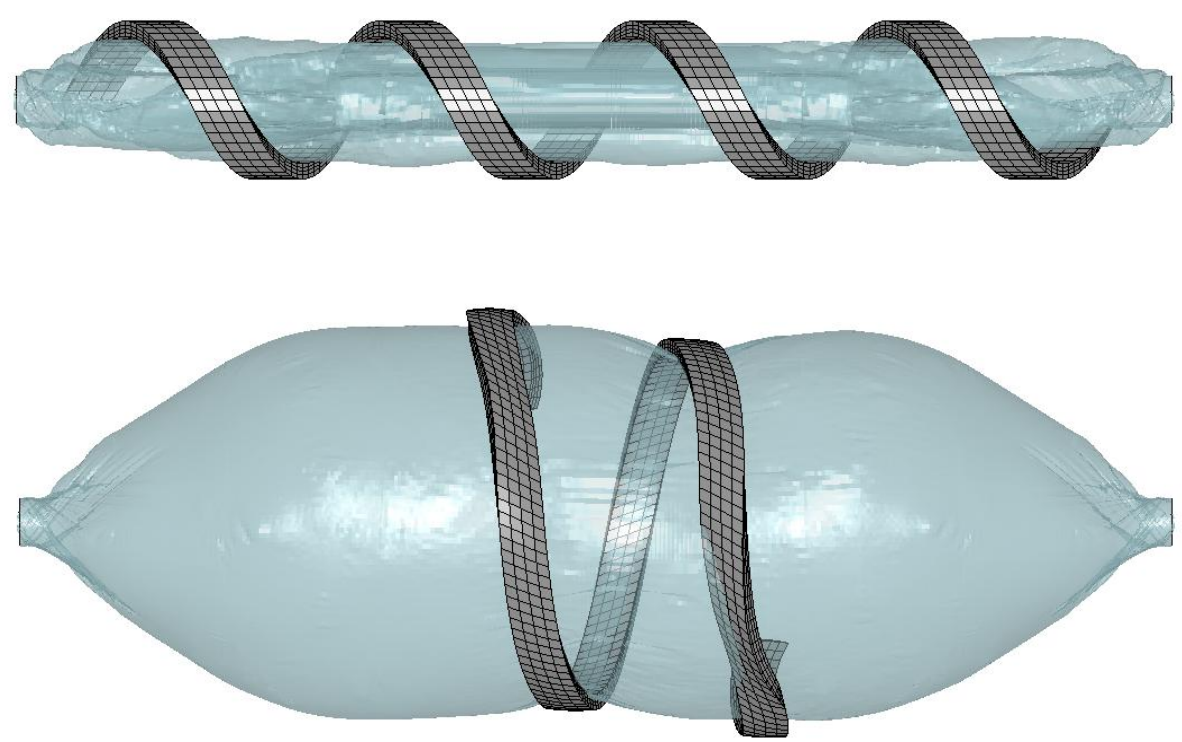

Figure 5-6 Expansion of a 0.24 mm (width) four-coil stent.

Expansion of a wider, $0.36 \mathrm{~mm}$, stent was also examined. At this width, the coils were not able to uncoil effectively; the stent showed asymmetrical expansion, dominated by the dog boning effect, which led to coil-overlap. The expansion model was terminated by the user upon first observation of coil-overlap (Figure 5-7). 

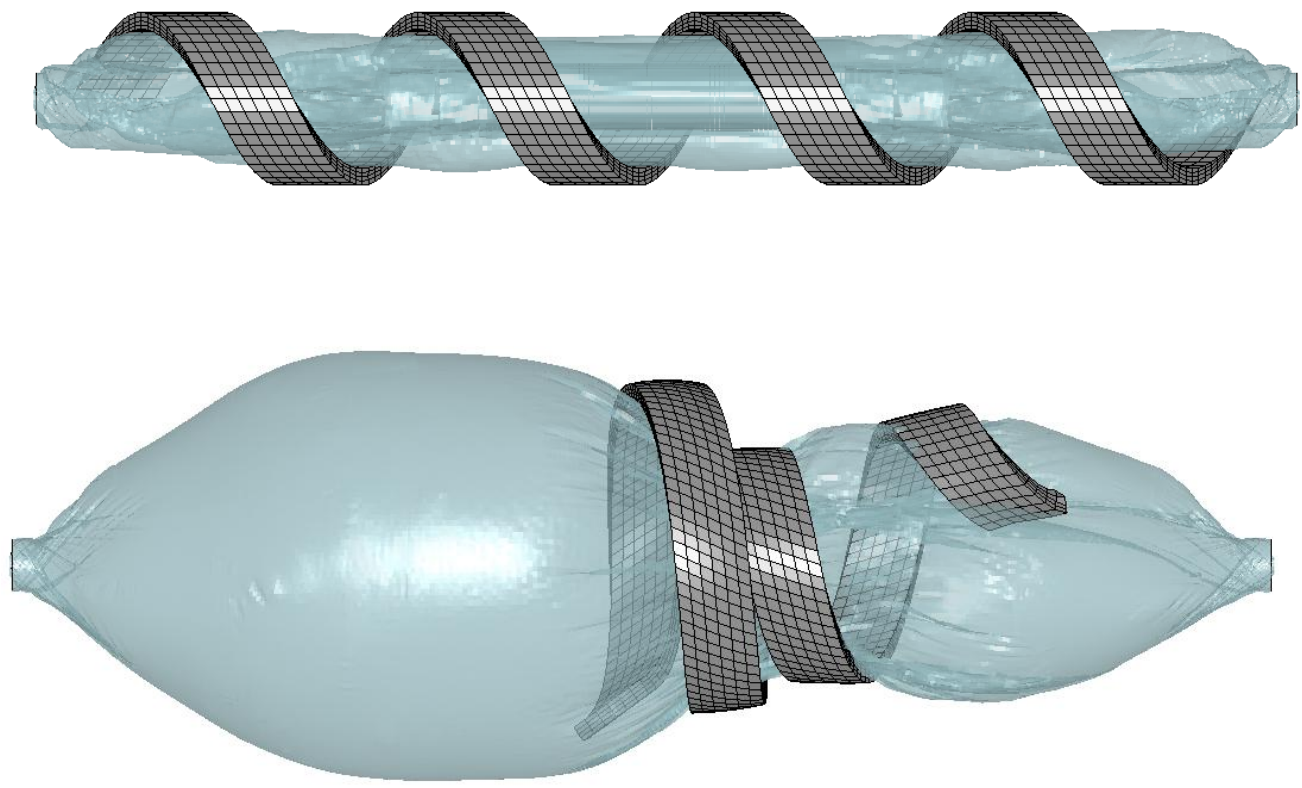

Figure 5-7 Expansion of a $0.36 \mathrm{~mm}$ (width) four-coil stent.

The study showed a clear trend towards asymmetrical and, ultimately, unsuccessful stent expansion with increased coil width; thus, larger coil widths (than $0.36 \mathrm{~mm}$ ) were not investigated. Helical stents with narrower coils, 0.12 and $0.24 \mathrm{~mm}$, were able to expand fully. Both stents showed small bending force, which facilitates increases in the radius of the coil, and frictional force at the stent-balloon interface. Therefore, when the balloon inflates, forcing the stent to increase in diameter, the tendency is for these stents to increase uncoiling behaviour rather than demonstrate changes in pitch value. These characteristics facilitate expansion along the balloon as it inflates, guarding against collapse and coil-overlap in the middle of the expanding balloon. Increasing coil width, however, tends to increase resistance to uncoiling, decrease the stent's pitch value during expansion, and encourage the coils to gather in the middle of the balloon, causing overlap. It should be noted, however, that although narrow stents can expand fully to the required diameter, they have small radial strength and tend to collapse under arterial wall pressure. 


\subsubsection{Effect of stent thickness}

Coil thickness is another factor that affects the bending strength of helical stents. Thin stents behave very similarly to the narrow stents studied in the previous section. The intention of this parametric study was to investigate expansion of four-coil stents ranging in coil thickness from 0.03 to 0.12 , beginning with the thinnest. A four-coil stent with $1.2 \mathrm{~mm}$ width, $0.03 \mathrm{~mm}$ thick coils, and a pitch value of $2 \mathrm{~mm}$ was expanded with a tri-folded balloon (Figure 5-8). The COF at the stent-balloon interface is 0.1 . This stent expanded successfully along with the balloon's inflation. The coils however, were very close and almost touched each other at the end of expansion. It was expected that a thicker stent, with higher dog boning effect, would not expand successfully; thus, thicker stents were not studied.
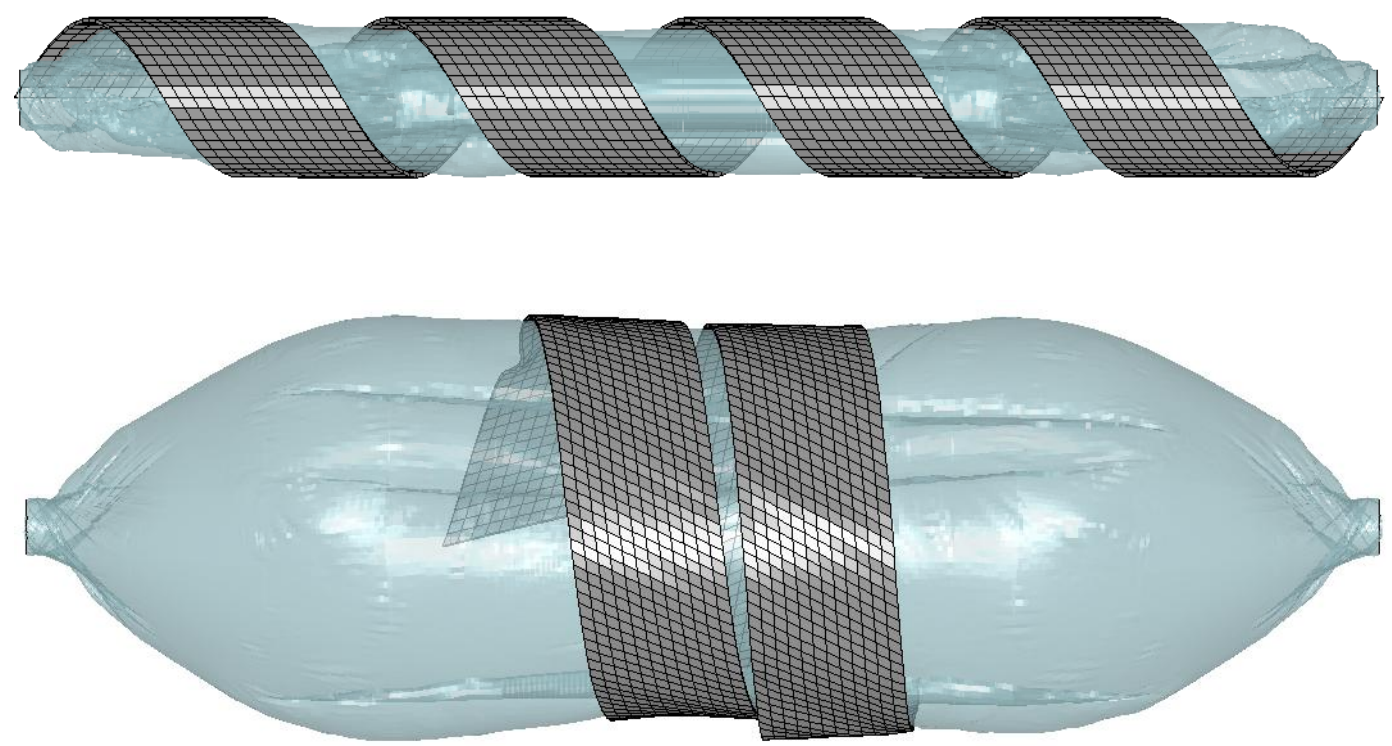

Figure 5-8 Expansion of a $0.03 \mathrm{~mm}$ (thick) four-coil stent.

Most of the parameters studied in the previous sections (5.1.1 and 5.1.2) represented global stent-balloon characteristics; that is, they described the mechanical properties of either the balloon or the stent as a whole. Dog boning, on the other hand, is a local problem, where the end-coils of the stent expand before the middle coils. Therefore, a method that increases the rate of expansion of the middle coils, to exceed that of the end-coils, would assist in supporting successful stent deployment. To that end, local changes can be made to the stent and balloon material, as well as the balloon and stent geometry. 
The level of structural deformation experienced when a force is applied depends on the structure's stiffness and boundary conditions. Therefore, in order to increase the rate of middle coil-expansion to surpass that experienced by the end-coils, one can either reduce the stiffness of the middle coils or apply greater force to them.

Decreasing the thickness of the middle coils also decreases their stiffness, enabling greater deformation under less pressure (than the end-coils). To investigate this phenomenon, a five-coil stent with various thicknesses along its length $(0.12 \mathrm{~mm}$ thick end-coils and $0.03 \mathrm{~mm}$ middle coils) was expanded (Figure 5-9). The stent was made of PLLA and had $1 \mathrm{~mm}$ wide coils, a pitch value of $2 \mathrm{~mm}$, and a $0.1 \mathrm{COF}$ at the stent-balloon interface. The balloon was divided into 7 segments, the two end cap segments and five additional segments each aligned with a stent coil.

As expected, the expansion model showed that the middle coils began expansion earlier than the end-coils; however, the dog boning effect and, consequently, the pushing force on the coils were very high, resulting in coil-overlap. The model analysis was terminated by the user upon observation of coil-overlap (Figure 5-9).

A separate parametric study was also undertaken to reveal the effect of the length of the low thickness portion of the stent on overall expansion. Through this study we found the best case for reducing the dog bone shape which is presented in Figure 5-9. 

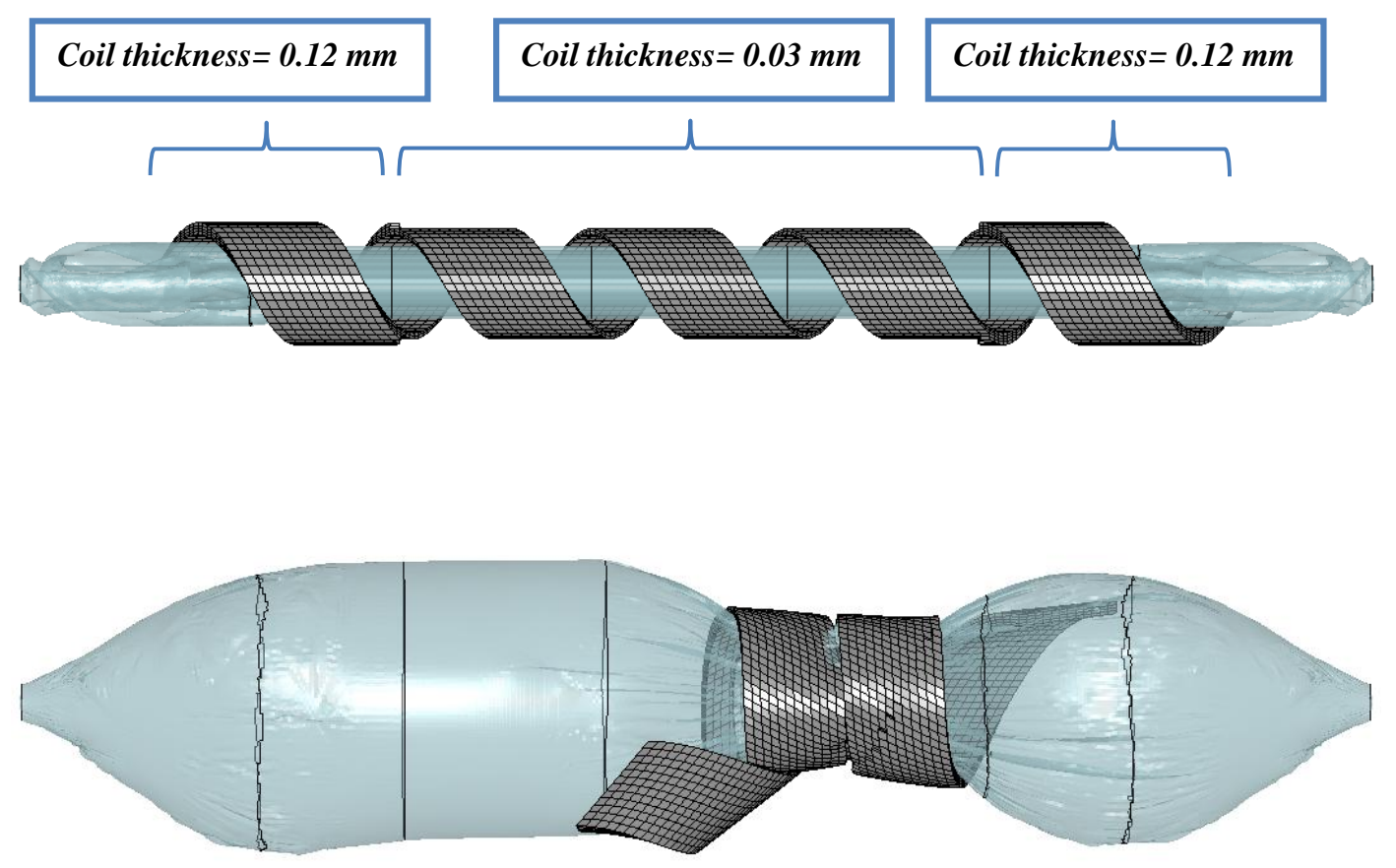

Figure 5-9 Expansion of a five-coil stent with varied thickness.

\subsubsection{Effect of stent pitch}

Pitch value is another geometrical factor that can affect the expansion of a helical stent. Stents with smaller pitch values have less surface area in contact with the balloon surface and consequently produce smaller frictional force during expansion.

The stent length is another important factor in stent expansion. In order to maintain the same stent length in this parametric study as that described in Section 5.1.3, the number of coils was increased as the pitch value of the stent was decreased. A five-coil stent with $0.03 \mathrm{~mm}$ thick and $1 \mathrm{~mm}$ width coils, a $0.1 \mathrm{COF}$ at the stent-balloon interface, and a pitch value of $1.2 \mathrm{~mm}$ was expanded with a tri-folded balloon (Figure 5-10). In the early stages of expansion, the initial dog bone shape of the balloon pushes the coils closer together. Furthermore, due to the smaller pitch, the initial distance between coils is smaller, causing the stent coils to overlap very early in the 
expansion process. The expansion model was terminated by the user at the first observation of coil overlap.
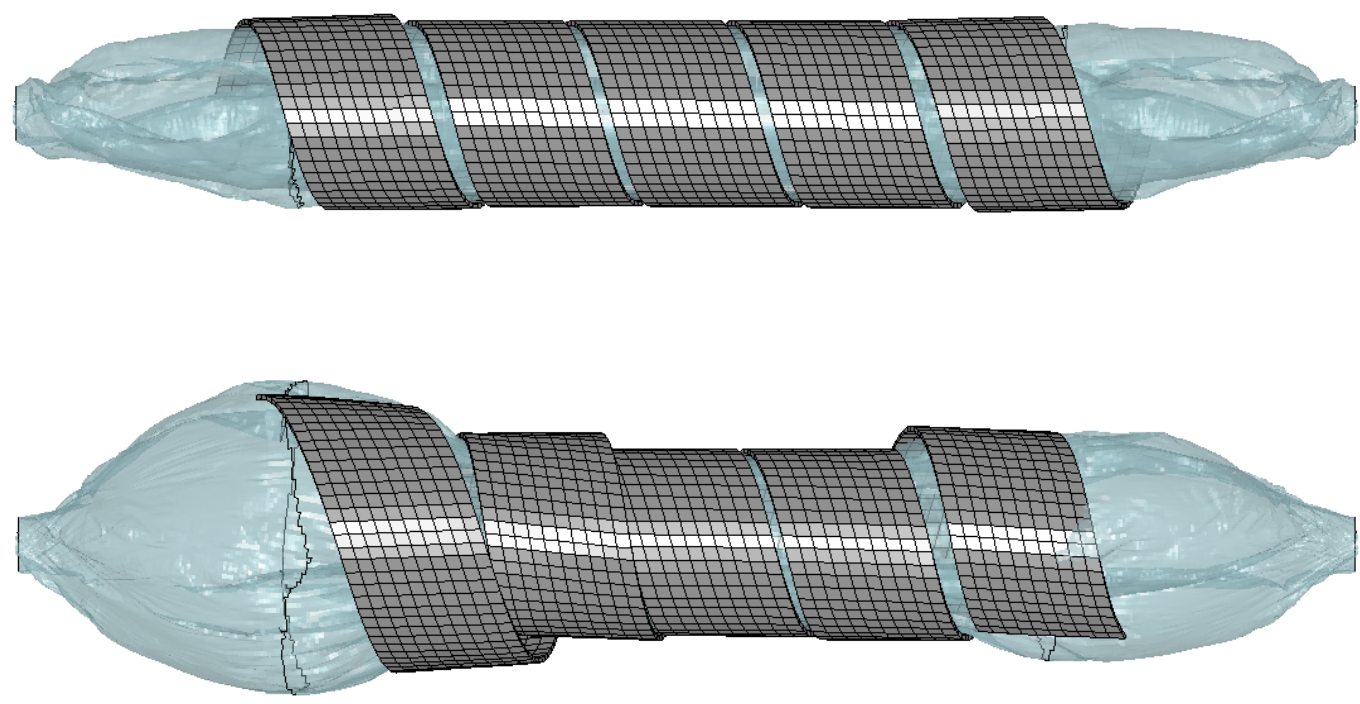

Figure 5-10 Expansion of a five-coil stent with pitch value of $1.2 \mathrm{~mm}$.

It is clear that in addition to stent geometry, the balloon's properties are also important to stent deployment. That is, the dog boning profile of the balloon (and consequently the stent) promotes coil overlap, which then terminates expansion. Thus, a method to effectively reduce dog boning in the expanding balloon may lead to successful expansion of the stent.

\subsubsection{Effect of number of balloon folds}

Balloon geometry, specifically the number of folds in the balloon, can be considered an important parameter for improving uniform stent expansion as it directly affects the stentballoon interface, which is the critical boundary condition in stent deployment. A six-coil helical PLLA stent with $1.2 \mathrm{~mm}$ width, 0.12 thick coils, $0.1 \mathrm{COF}$ at the stent-balloon interface, and a pitch value of $2 \mathrm{~mm}$ was used to study the impact of number of balloon folds (Figure 5-11). The number of coils was selected to ensure that the length of the balloon matched the length of the expanded stent. The balloon used in this study had four folds and was developed using the same 
method as that used to design the tri-folded balloon described in each of the other studies. The results showed that inflation of this type of balloon produced the same asymmetrical expansion and dog bone shape as the tri-folded balloon expansion described earlier. That is, the dog boning effect pushes the stent towards the middle of the balloon, where the coils overlap, terminating the expansion process.
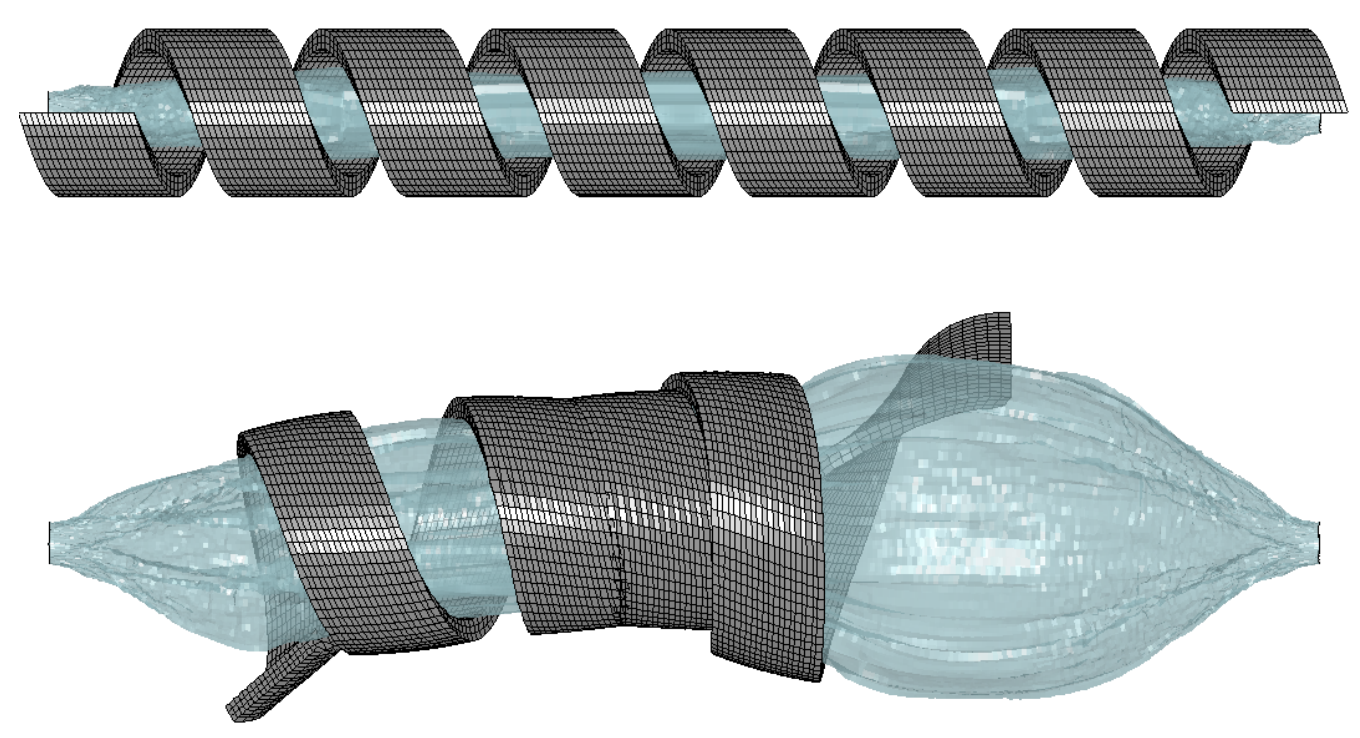

Figure 5-11 Expansion of a six-coil stent using a balloon with four folds

\subsubsection{Effect of balloon compliance}

Attempts at uniform expansion have shown that, if the dog boning effect is not produced, helical stents can expand to the required diameter. Therefore, efforts to make the balloon stiffer, so that it behaves similarly to a high stiffness cylinder, may support uniform and successful stent expansion. Balloons made with stiffer materials are called "non-compliance balloons" in the market. The balloon's stiffness should be sufficient to minimize the impacts of stent expansion on balloon expansion. This suggests that thin stents, which have low radial resistance, may be an ideal option. 
This option was evaluated by expanding a four-coil helical stent using a tri-folded balloon with a Young's modulus of $0.5 \mathrm{GPa}$ (Figure 5-12). The PLLA stent had a thickness of $0.03 \mathrm{~mm}$, width of $1 \mathrm{~mm}$, and pitch value of $2 \mathrm{~mm}$.

Expansion of the non-compliance balloon was more uniform than with the previously studied semi-compliance balloons. However, asymmetrical expansion occurred at one end of the stent faster than the other and dog boning caused the stent coils to overlap, which causes element distortion and subsequent termination of the analysis.
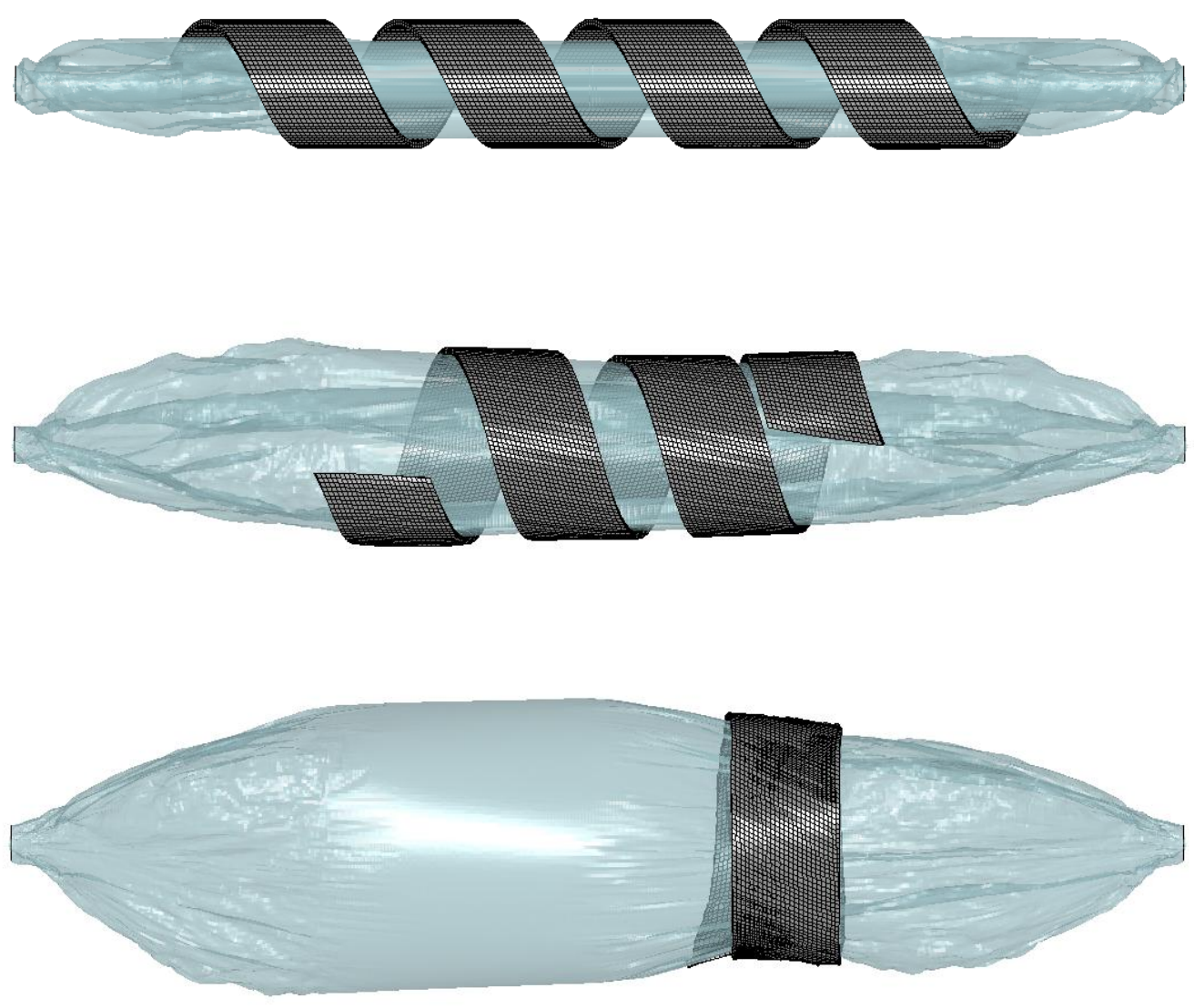

Figure 5-12 Expansion of a four-coil stent with a non-compliance balloon.

Due to the very thin wall of the balloon, increasing material stiffness cannot greatly impact the balloon's strength. However, adding thickness to the balloon's wall to increase its strength is not 
an option since the balloon must remain sufficiently flexible to fold into a very small diameter. Furthermore, in comparison, the stent will always be stiffer than the balloon, owing to its stiffer material and thicker structure.

There are several ways to reduce loading on specific parts of the stent. As the balloon wall is the medium to apply pressure to the stent, by changing the geometry or the mechanical properties of the balloon, the applied load to the stent is changed. This was evaluated by varying the balloon's wall thickness between its end (i.e., the end-cap segments, extending a short distance towards the middle of the balloon) and middle segments. Specifically, the balloon wall was thickest at its two ends, roughly 6 times that of the middle segments (i.e., $0.005 \mathrm{~mm}$ (middle) and 0.03 (ends)). Since thinner stents tend to demonstrate more uniform expansion, they were considered the best option for this case study. Furthermore, models of thin stents have fewer elements and are faster to run. Therefore, a thin, five-coil PLLA stent with a thickness of $0.03 \mathrm{~mm}$, width of $1.2 \mathrm{~mm}$, and pitch value of $2 \mathrm{~mm}$ was used. The COF at the stent-balloon interface was 0.1. By increasing the balloon's thickness at its two end-caps, this portion of the balloon tends to expand under higher pressure levels than the middle segment (Figure 5-13). From the outset, the expansion model showed a tendency towards dog boning and overlap at the end-coils of the stent (Figure 5-13), and was therefore terminated by the user.

A separate parametric study was undertaken to determine the effect of the length of high strength segments of the balloon on stent expansion. Here, we have presented the typical expansion resulting in the dog bone shape in Figure 5-13. 


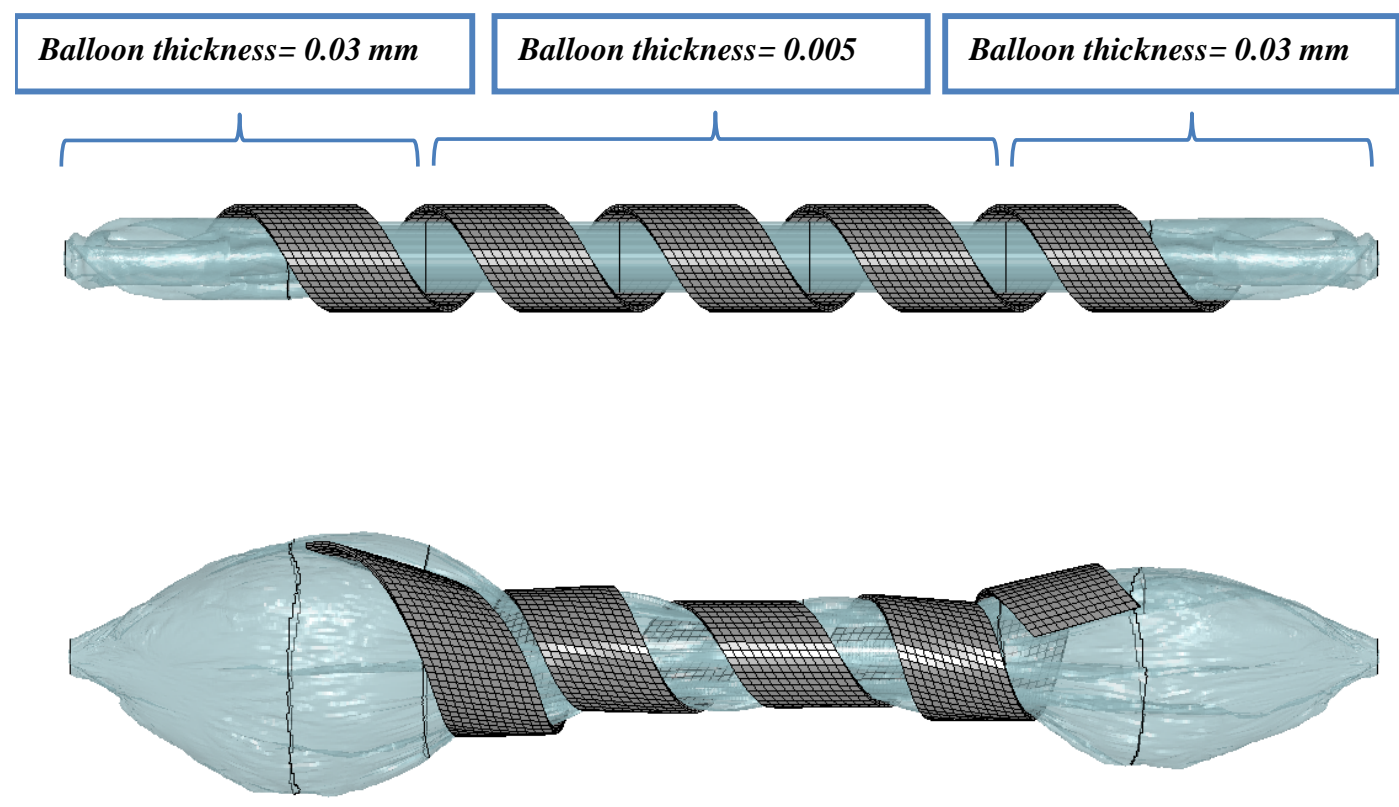

Figure 5-13 Expansion of a five-coil stent using a tri-folded balloon with varied wall thickness.

\subsubsection{Effect of balloon length}

It has been reported that dog boning decreases as the balloon length shortens [Kiousis, 2009], i.e., the full balloon length is shorter than the non-expanded stent. This effect was examined by expanding a five-coil helical PLLA stent with $1 \mathrm{~mm}$ wide, 0.12 thick coils, and a $2 \mathrm{~mm}$ pitch value (Figure 5-14). The balloon used to expand the stent was tri-folded and the COF at the stent-balloon interface was 0.1. During expansion, dog boning occurred and the expanded portion of the balloon penetrated between the coils, which not only pushes the middle coils towards the middle of the balloon, causing overlap, but also increases the pitch value at the stent's end-coils. Although the middle coils overlap, the outer coils slide off the end of the balloon during expansion. Since full expansion of the stent was impossible, the analysis was terminated by the user (Figure 5-14). 

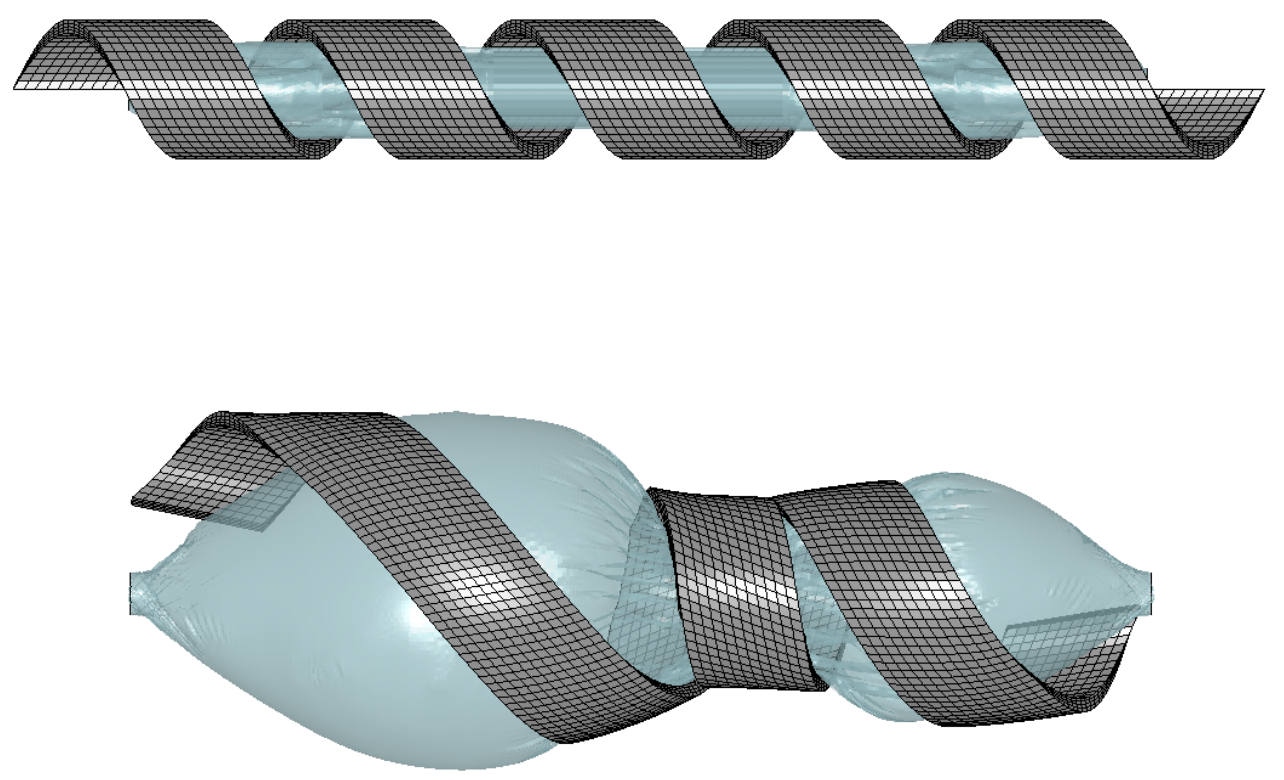

Figure 5-14 Expansion of a four-coil stent with a short balloon.

As previously mentioned, stent-balloon expansion studies described in the literature, which typically examine wire mesh stents, have shown that dog boning is minimized when the balloon is shorter than the stent. This study, however, showed that this phenomenon does not follow for helical stent expansion. The difficulty arises from the use of coils in helical stent design, where the expanded portion of the inflating balloon can penetrate between the coils and push the dog bone shape towards the middle portion of the stent. Formation of the dog bone shape was later followed by overlap of the middle coils and the end-coils slid off the end of the balloon, preventing full expansion of the stent.

\subsubsection{Effect of COF at the balloon-stent interface}

The frictional force at the interface between the stent and balloon is an important factor in altering the stent's resistance to uncoiling during expansion. For example, by changing the balloon material, the $\mathrm{COF}$ at the balloon-stent interface changes; as friction at the interface increases, it becomes harder for the coils to slide over the balloon and overlap.

This effect was examined with a four-coil stent that has $1 \mathrm{~mm}$ wide, $0.12 \mathrm{~mm}$ thick coils, and a pitch value of $2 \mathrm{~mm}$. The stent was expanded with inflation of a tri-folded balloon (Figure 5- 
15). To study the effect of COF on helical stent expansion, the COF at the balloon-stent interface was set to 0.2 , which is slightly higher than the current COF. This COF value does not have a specific physical interpretation; however, some of the balloon wall material can have this COF. During expansion, the inner coils of the stent overlapped and the analysis was terminated by the user.

The results show that although increased friction reduces the ability of the stent coils to slide over the balloon, it also limits uncoiling behaviour, which in turn promotes development of the dog bone shape in the balloon. By increasing the dog boning effect, the tendency for the middle coils to be pushed towards the middle of the balloon, where they overlap, is also increased.
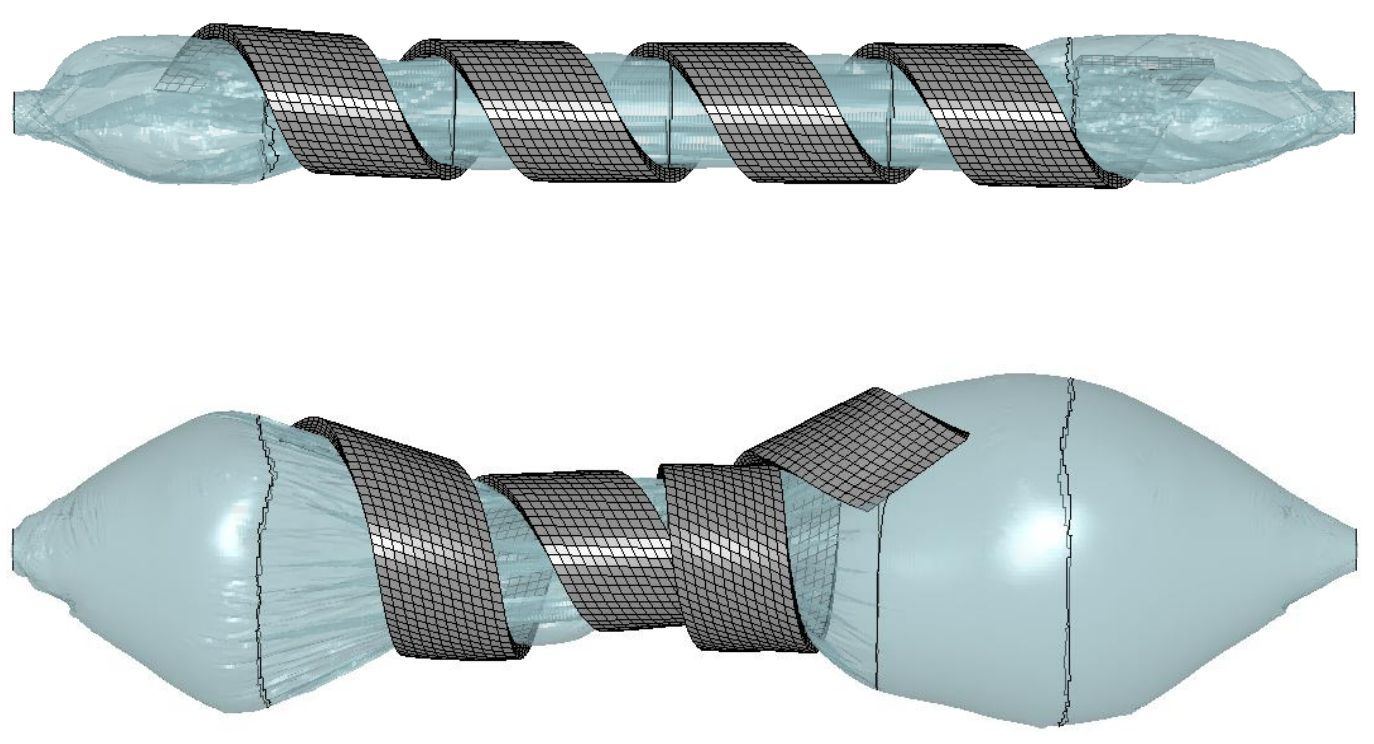

Figure 5-15 Expansion of a four-coil stent with $\mathrm{COF}=0.2$ at the stent-balloon interface.

It can be concluded from this study that higher COF values, which reduce uncoiling behaviour, cannot solve dog boning and coil-overlap issues. However, we also know from the uniform expansion study described in Chapter 3, where the COF was 0 , that low COF values reduce the stent's pitch, which does not resolve challenges to full expansion.

Varying material properties of the balloon not only affects the stent-balloon interface, it can affect the stiffness of the balloon wall as well. To examine the effects of balloon stiffness on stent expansion, the modulus of the end segments of a tri-folded balloon was increased 50 times 
(Figure 5-16) and 100 times (Figure 5-17). The balloon was then used to expand a five-coil PLLA stent with $1.2 \mathrm{~mm}$ wide, $0.03 \mathrm{~mm}$ thick coils, and a pitch value of $2 \mathrm{~mm}$. The COF at the stent-balloon interface was 0.1 .

By increasing the stiffness of the balloon at both ends (i.e., the cap segment, extending a short distance towards the middle of the balloon), these segments tend to require higher pressure for expansion than the middle segments.
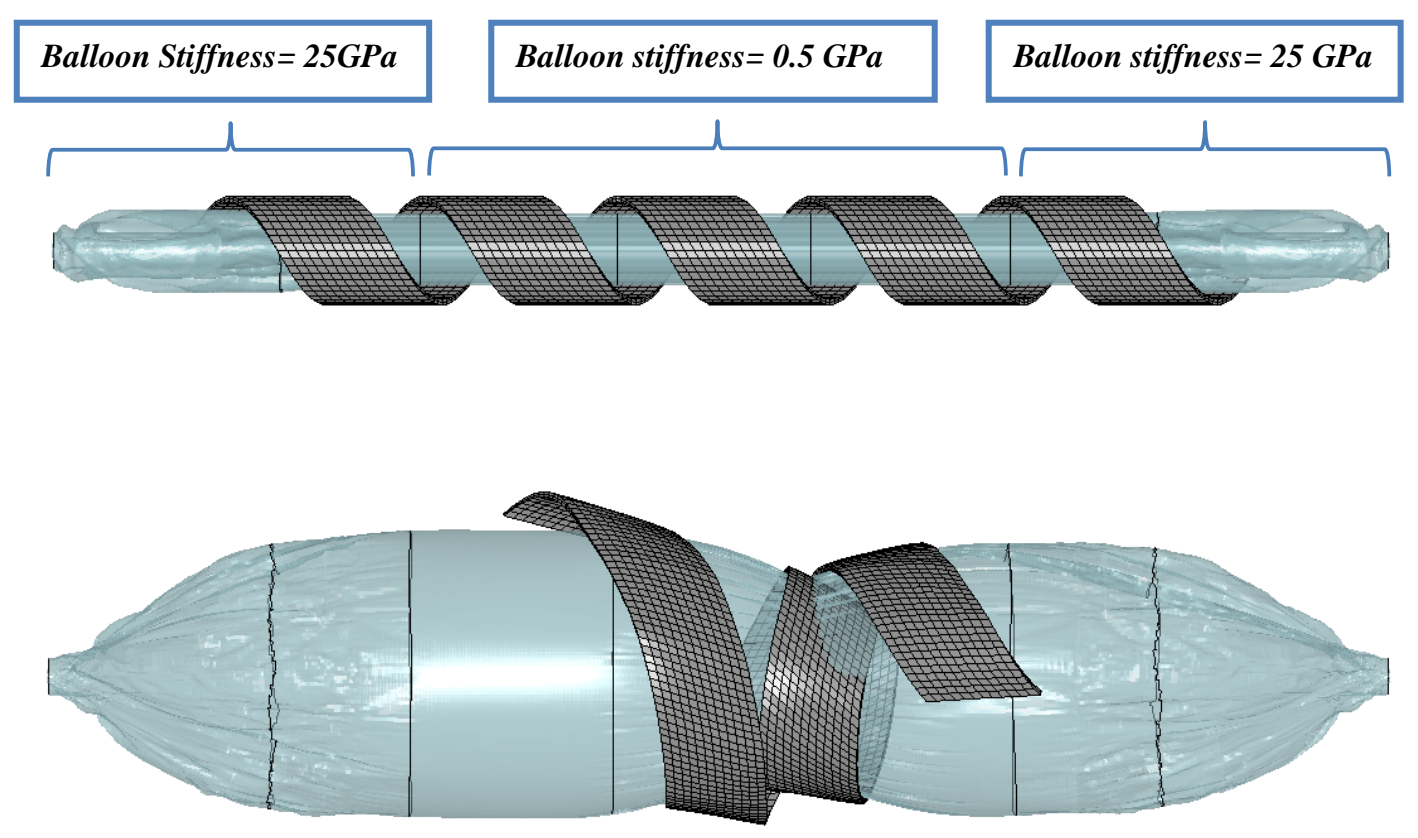

Figure 5-16 Expansion of a five-coil stent with a balloon bearing 50 times the modulus at its end cap segments.

Increasing Young's modulus 50 times changed the expansion profile, however, it was not enough to eliminate dog boning or coil-overlap issues (Figure 5-16). Upon first observation of coil-overlap, the model analysis was terminated by the user. 

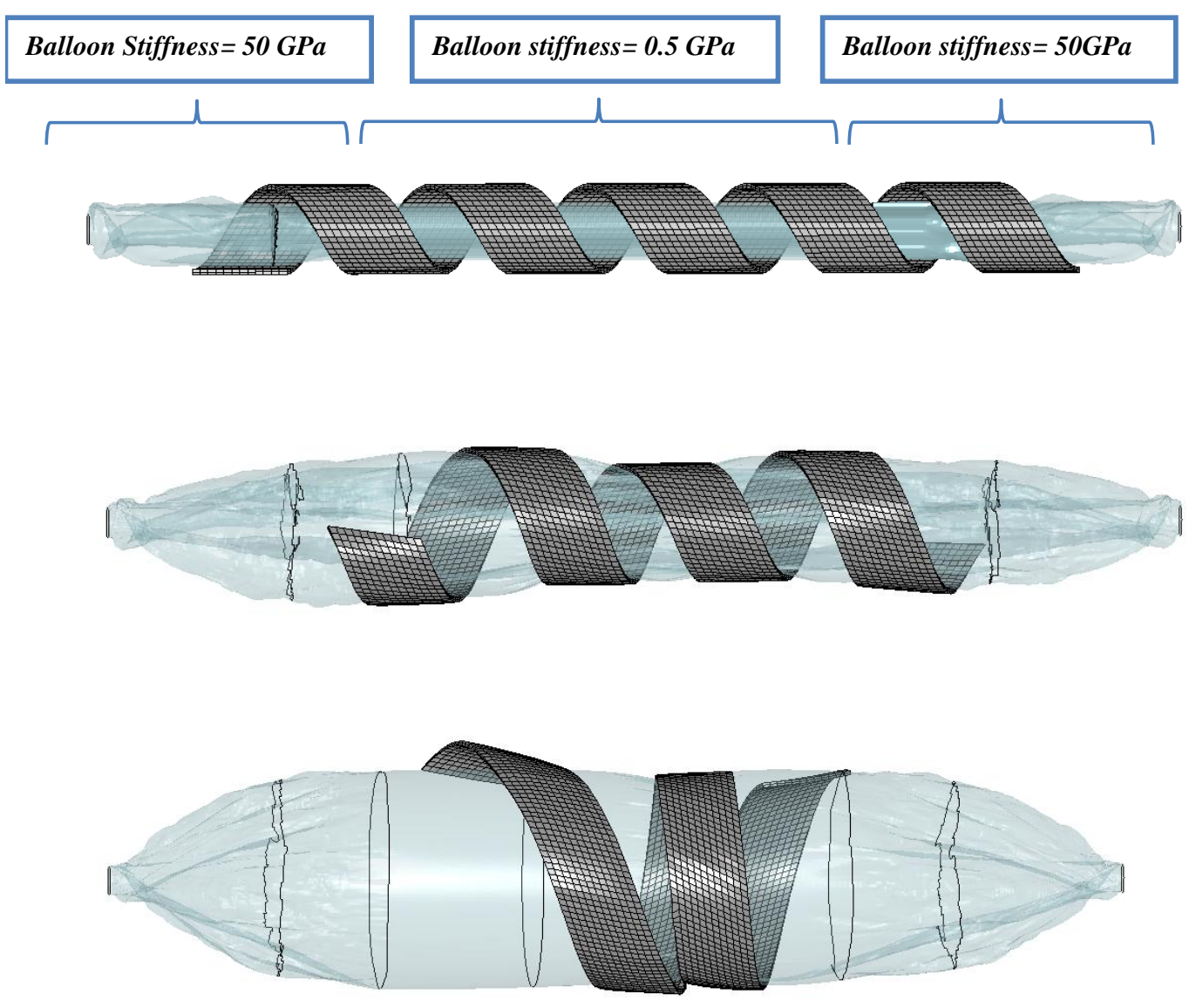

Figure 5-17 Expansion of a five-coil stent with a balloon bearing 100 times the modulus at its two end cap segments.

Further increasing the modulus of the end-segments to 100 times that of the middle segments enabled successful stent expansion. The stent reached its required diameter and overlapping was only observed in the final stages of expansion (Figure 5-17).

Although a thin helical stent can be successfully expanded with a folded balloon, it cannot provide sufficient radial support for clinical application. Successful expansion depends on the stiffness ratio between the balloon and the stent structure. Based on the current study, in order to achieve successful expansion of a clinically viable stent design (i.e., using a $0.12 \mathrm{~mm}$ thick stent), the end segments of the balloon should be more than 100 times stiffer than the middle 
segments. This high stiffness portion provides enough stent-balloon stiffness ratio to expand the stent successfully.

\subsection{Alternative stent designs}

Up to this point, the entire focus of this research has been placed on improving stent expansion by adjusting the mechanical properties of the stent components and/or balloon; however, the overall geometry and design of the stent itself can be considered an important factor in its expansion.

A major difficulty in helical stent expansion is coil-overlap following dog-bone shape development in the expanding balloon. This stems from the fact that helical stents demonstrate minimal axial resistance, which means they cannot prevent coils from sliding back towards the middle of the balloon when dog boning occurs in the cap segments. Therefore, new stent designs that incorporate axial links to limit axial coil movement may be helpful in overcoming this challenge. Three new stent designs were considered and are discussed below.

\subsubsection{Alternative stent design I: helical stent with links}

The first attempt to eliminate coil-overlap through enhanced axial resistance produced a helical stent design with links added in the axial direction, between every second coil (Figure 518). The resulting four-coil stent had $1 \mathrm{~mm}$ wide, $0.12 \mathrm{~mm}$ thick coils, and a pitch value of 2 $\mathrm{mm}$. The link thickness was equal to that of the neighboring coils. 

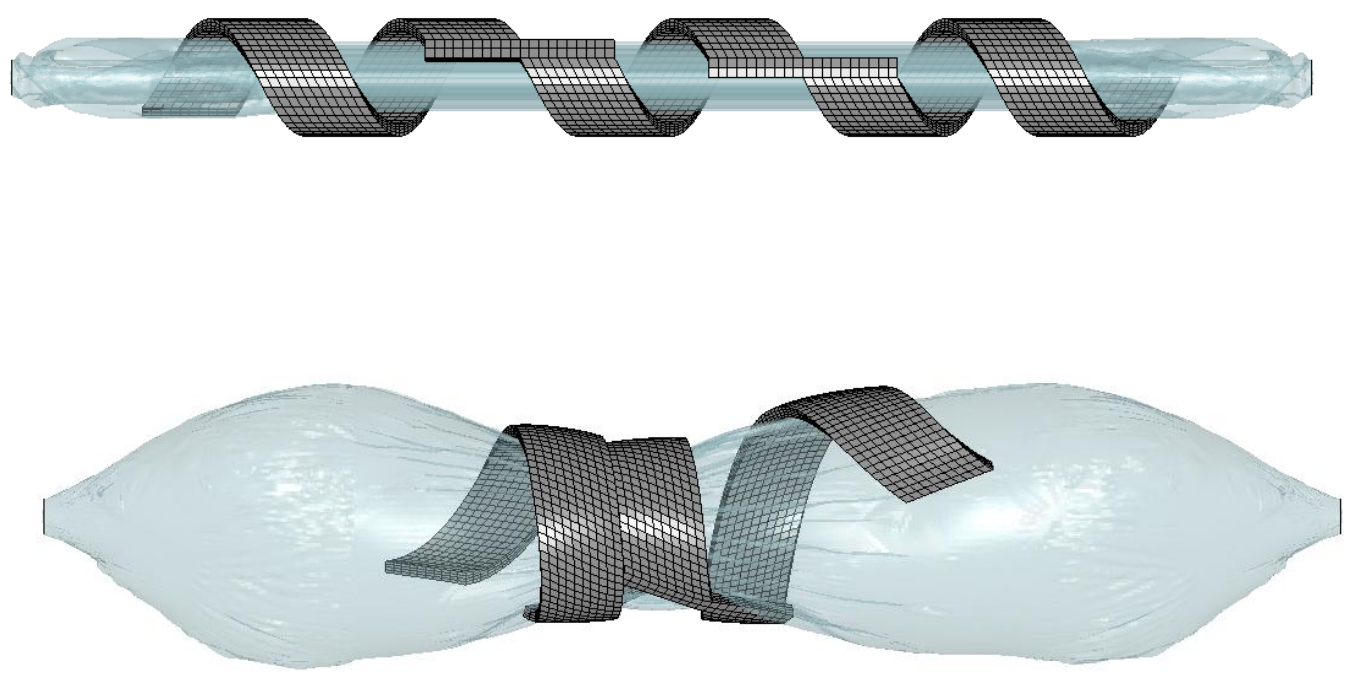

Figure 5-18 Expansion of alternate stent design I: a four-coil helical stent with axial links.

The simulation showed that while the links limited coil-overlap somewhat, they could not eliminate it completely. Foreshortening and dog boning behaviour were similar to that seen in the original helical stent seen in Section 5.1.1. The number of coils decreases during expansion because of the uncoiling mechanism. Therefore, the links that originally connected coils, and were intended to prevent overlap, shifted one-by-one to the middle of the half expanded coils. During expansion, and due to dog boning, the coils slid closer together and the links overlapped with the neighboring coils. Thus, this design did not support successful stent expansion.

\subsubsection{Alternative stent design II: helical stent with three sequentially connected two-coil sections}

In Section 5.1.1, which evaluated the effect of coil number on stent expansion, it was observed that two-coil stents can effectively expand to requisite diameters. This suggests that a sequence of two-coil stents may be equally successful. For example, by adding two-coil stents in sequence as shown is Figure 5-19, expansion of the two end coils draws the middle coils distally, away from the center of the balloon, supporting expansion. Therefore, expansion of the middle coils is facilitated by the end-coils and more uniform expansion is achieved. 

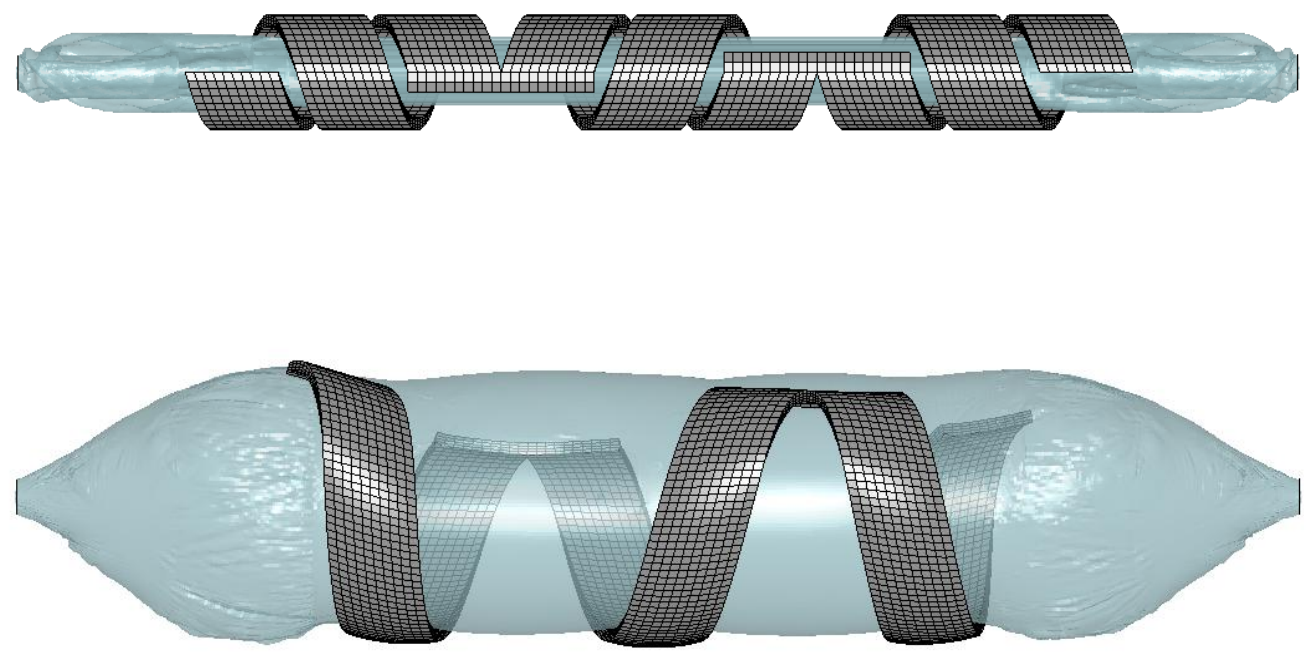

Figure 5-19 Expansion of alternate stent design II: helical stent design with three sequentially connected two-coil sections (totaling six-coils).

By connecting the two-coil sections together, expansion of the end sections forces the middle section to uncoil. Therefore, during expansion of a three section stent (six-coil stent), the two end sections expand the middle one, thus eliminating the dog boning effect. However, the uncoiling factor was only about $60 \%$, which means the two middle coils expanded to less than one coil. The fully expanded stent cannot cover the full cylindrical surface of the balloon, which means this design can only offer partial coverage of the artery and cannot provide suitable radial resistance of the artery. Therefore, despite its success in expansion, this design cannot support full coverage of the artery post-expansion (Figure 5-19). Furthermore, the links between coil sections produce stress concentration points in the stent. This may be a significant problem as high stress areas dissolve at a faster rate and may flake away pieces of the stent into the artery, blocking the flow of blood.

\subsubsection{Alternative stent design III: three-coil sequential helical stent}

To solve the coverage problem of stent design II, a similar stent design was developed, but with three sequentially connected three-coil sections, totaling nine coils (Figure 5-20). 

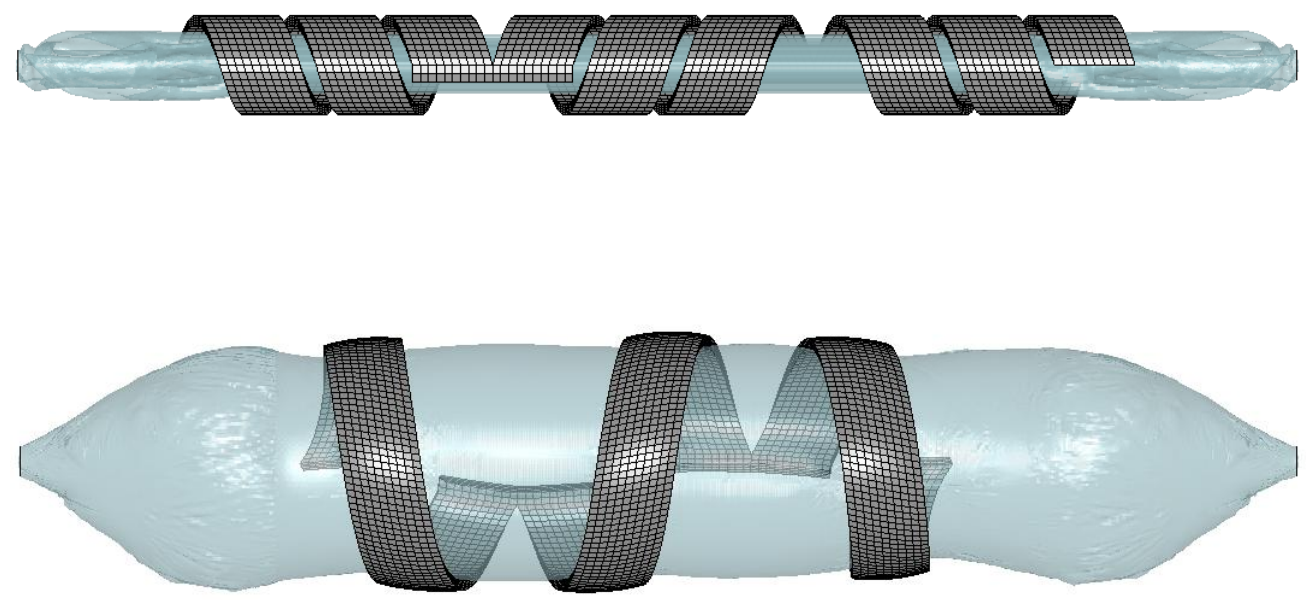

Figure 5-20 Expansion of alternate stent design III: helical stent with three sequentially connected three-coil sections (totaling nine coils).

This stent did not demonstrate dog boning or asymmetrical expansion issues. It also achieved $100 \%$ circumferential coverage after expansion. However, the only drawback of this stent design is the fact that the application of radial force (such as the pressure of artery) will expose the expanded stent to potentially immitigable bending force. That is, since the weakest points of this structure all fall on one side of the stent (Figure 5-20), application of arterial/plaque loads may cause the middle portion of the stent to bend and ultimately break. Stress concentrations at the joints also represent potential breakage points under artery/plaque pressure. Due to the degradation behaviour of PLLA, these high stress points tend to dissolve faster than other segments of the structure, which causes small pieces of stent to enter into the blood stream, increasing the risk of blockage.

\subsection{Progressive pressurization}

Another way to support improved stent expansion is to apply greater pressure to the middle coils of the stent at an earlier stage of the expansion process. By applying differential, but tightly controlled, pressure from the inflating balloon, the stent can be made to begin expansion at its middle coils and progress towards its ends. A series of parametric studies was undertaken to find a suitable balloon expansion pattern to support this expansion. A five-coil stent with $1 \mathrm{~mm}$ wide, 
$0.12 \mathrm{~mm}$ coils, a pitch value of $2 \mathrm{~mm}$, and $\mathrm{COF}$ of 0.1 at the stent-balloon interface was expanded with a tri-folded balloon (Figure 5-21). The inflation pattern used was asymmetrical at the balloon's ends to compensate for the asymmetrical expansion of typical helical stents.
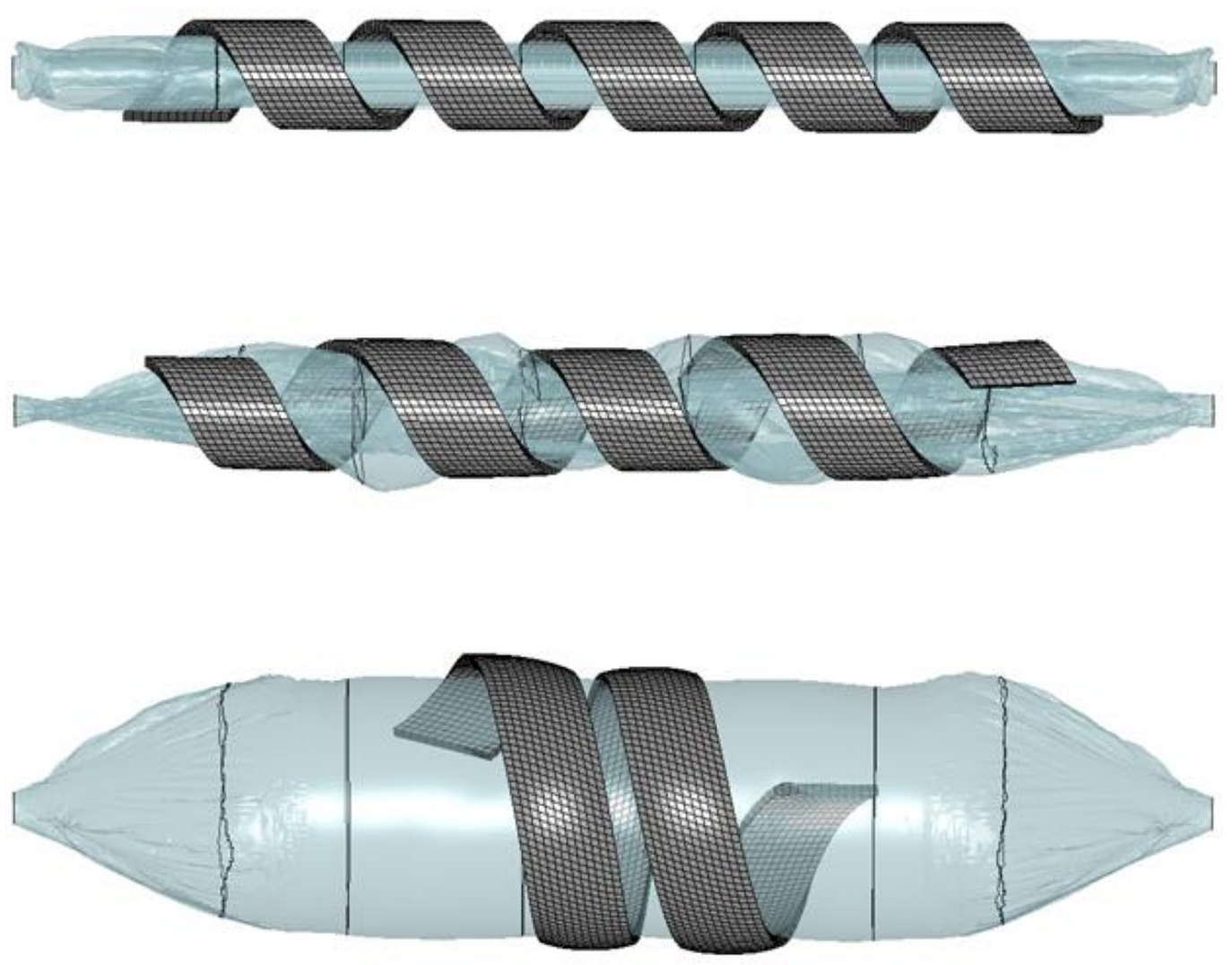

Figure 5-21 Expansion of a five-coil stent with a tri-folded balloon using a progressive pressurization pattern.

To address the dog boning issue, a progressive balloon expansion method was investigated allowing for expansion in a controlled manner. The body of the balloon was separated into five regions, corresponding to the five coils of the stent. Pressure was first applied in the central zone of the balloon (i.e., the middle coil of the stent), and, as expansion proceeded, continued to be applied to the inner surface of the balloon, expanding progressively from the mid-section distally towards the two ends. This progressive pressurization pattern was successful; only a modest 
amount of dog boning was observed and the coils did not slide toward the center of the balloon. This means the coils did not overlap, allowing the stent to expand fully to the required diameter (Figure 5-21).

The ability to successfully support an artery following stent deployment directly depends upon the stent's radial strength and the amount of recoil or elastic recovery it demonstrates postexpansion [Paryab 2014]. These factors are in turn dependent upon the level of plastic strain experienced by the stent material through the expansion process. In Chapter 4, a uniform expansion approach predicted a maximum plastic strain of $2.7 \%$ in the helical coil stent, which resulted in approximately $10 \%$ radial recoil. By comparison, the stent progressively expanded with the folded balloon had a maximum equivalent plastic strain of 5\% (Figure 5-22) and demonstrated reduced recoil behaviour. Further, the strain distribution was relatively uniform with a local maximum in the latter example (Figure 5-22), which is desirable for a stent. The local maximum is in the center of the middle coil of the stent, which is consistent with the circumferential stress distribution (which depicts the highest stress).

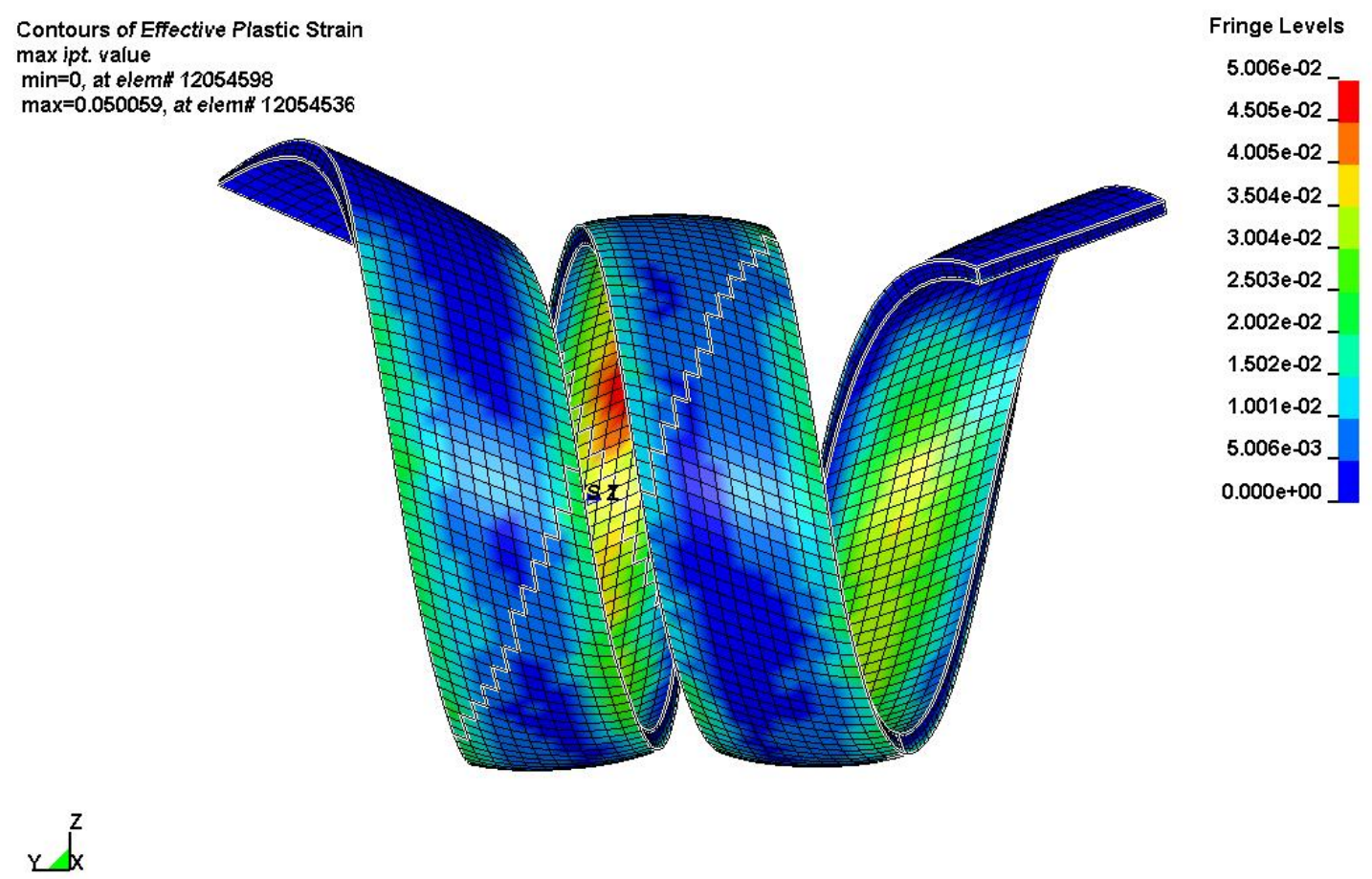

Figure 5-22 Equivalent plastic strain distribution for a 3mm radial expansion using the progressive balloon expansion approach. 
One difference noted between uniform and balloon expansion was the cross-sectional shape of the expanded coils (Figure 5-22), where the balloon-expanded coils was convex in shape. This effect could be due to the fact that the balloon material is more compliant than the stent. This type of deformation has not been observed in the uniform expansion model, as the expanding cylinder is high stiffness and forces the stent to expand in the radial direction. In actual practice, the convex deformation may be important in reducing the stresses on the artery, and will be the subject of a future investigation.

The circumferential (hoop) stress distribution on the stent at the maximum diameter is also uniform with the maximum tensile stress distribution in the inner surface and the compressive stresses at the outer surface. The maximum tensile stress (at the inner surface) is higher than the maximum compressive stress (at the outer surface). This is a typical stress distribution in the curved membrane (i.e., different levels of tensile and compressive stresses). This kind of stress distribution in a curved membrane suggests that the bending is one of the deformation modes in the structure and the curvature of the membrane increases during deformation.

The maximum stress area is located at the inner surface of the middle coil of the stent. The maximum compressive stress, however, is at the two edges of the middle coil. The stress at the edges (and vicinity of the edges) of the stent is almost zero.

Along the width of each coil (at the same radial distance from the middle axes of the stent) the stress distribution is symmetrical. For instance, the stress starts from a maximum on the outer surface of the middle coil and gradually drops toward the coil's middle, and then increases again to a similar maximum at the starting corner. This kind of stress distribution suggests that the pitch angle of the stent has minimal effect on the stress distribution and also that the stent does not possess axial restrictions that hold it in the initial pitch angle. 


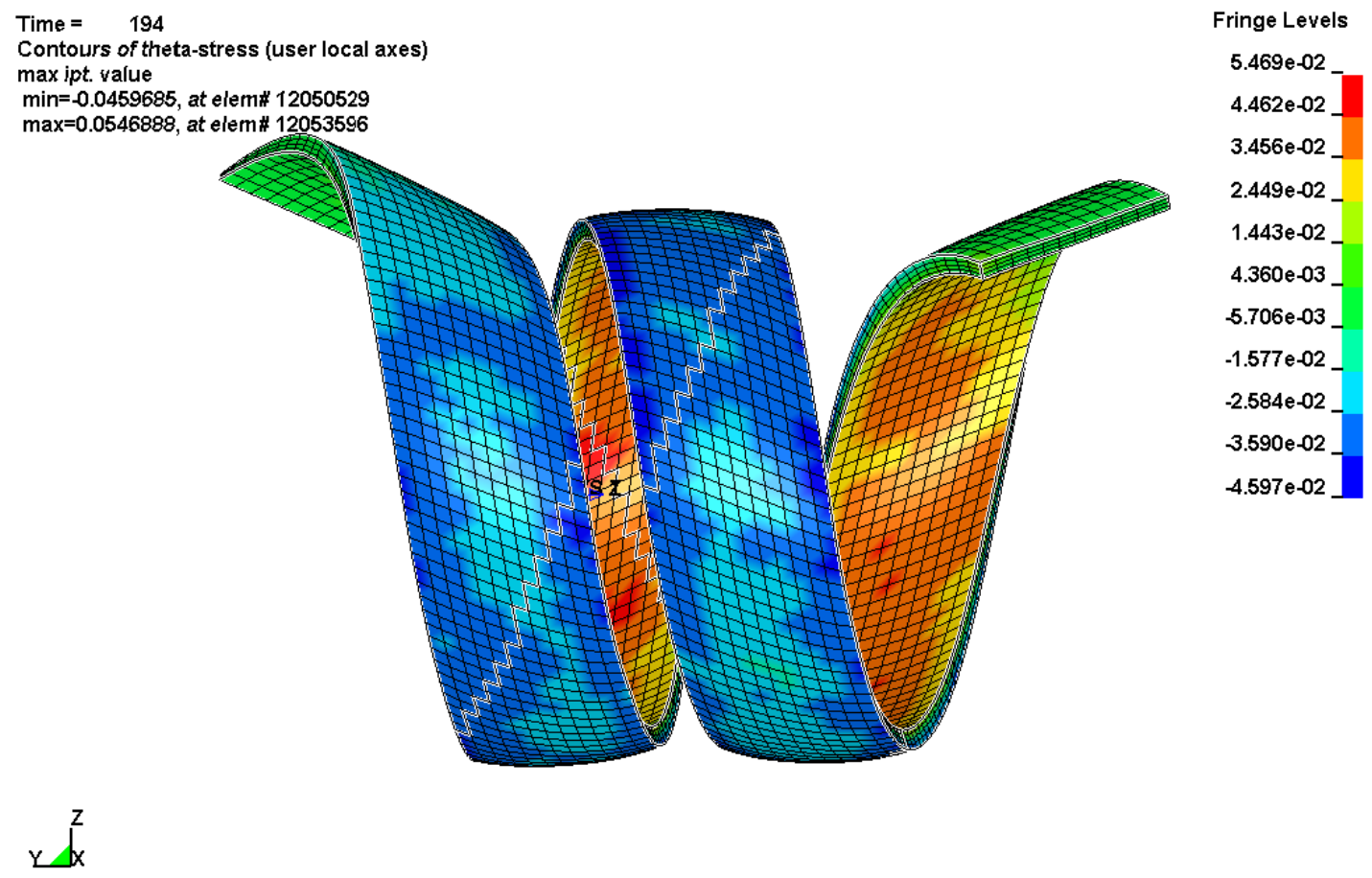

Figure 5-23 Circumferential stress distribution (GPa) in a helical stent expanded to $3 \mathrm{~mm}$ diameter (without the surrounding artery).

The axial stress distribution in the stent is also uniform (Figure 5-24), with localized maximum stress at the two edges of the stent. The stress levels are almost zero at the corners of each coil. There is tensile and compressive stress at the middle of each coil. This distribution is due to the curvature of the coils. Each coil has an outward curvature in the axial direction which is compressive at the outer surface and tensile at the inner surface. 


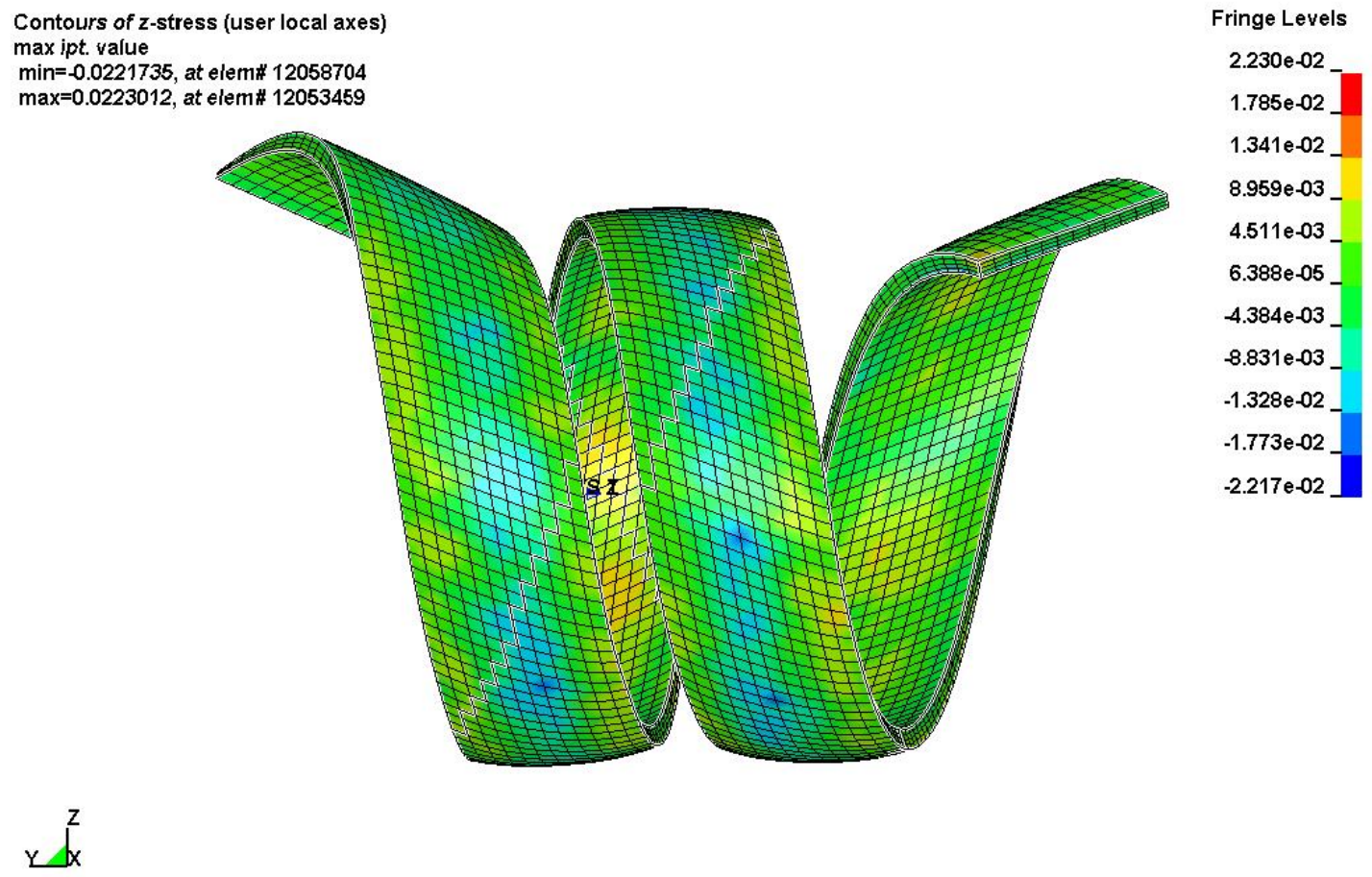

Figure 5-24 Axial stress distribution (GPa) in a helical stent expanded to $3 \mathrm{~mm}$ diameter (without the surrounding artery).

The circumferential, axial, and radial stress distributions of the helical stent after expanding to the outer diameter of $3 \mathrm{~mm}$ were plotted (Figures 5-23, 5-24 and 5-25). The radial stress distribution in a freely expanding stent (without the surrounding artery) is uniform with localized stress concentrations at the edges of the stent (within a few elements). Except for the edges, the stress levels are negligible (almost zero). This kind of stress distribution in a bent membrane suggests that there is no radial resistance in the deformation and the membrane has thin walls. All of these conclusions are consistent with the applied boundary conditions and the loading and geometry of the stent. 


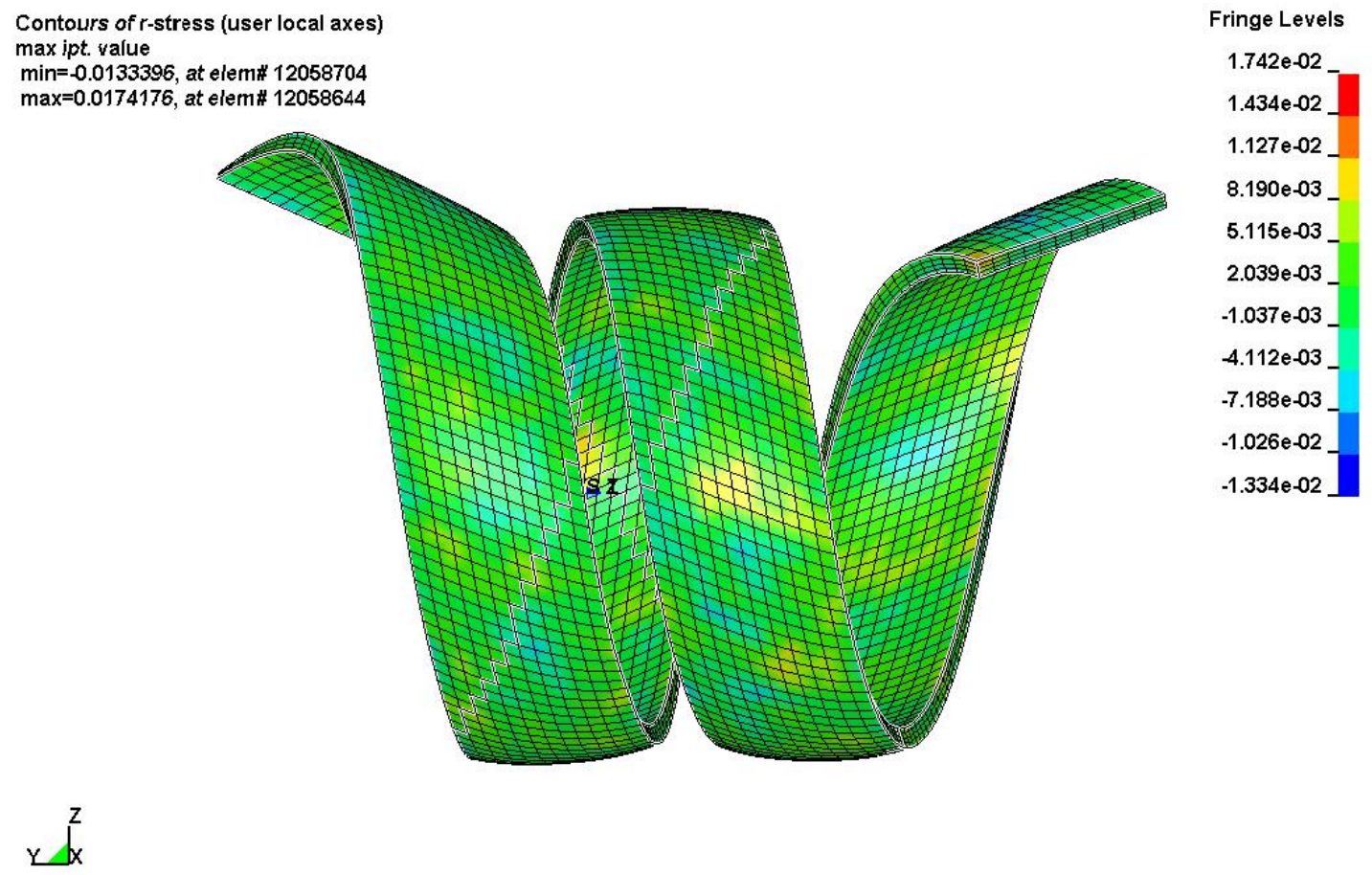

Figure 5-25 Radial stress distribution (GPa) in a helical stent expanded to $3 \mathrm{~mm}$ diameter (without the surrounding artery).

A parametric study was conducted on the effects of COF at stent-balloon interface on the dog boning. The results showed that a significant increase in the $\operatorname{COF}(0.0,0.1$, and 0.2$)$ between the stent and balloon reduced dog boning by a modest amount but did not significantly affect the results as the high COF values applied would be unrealistic for typical stent and balloon materials (Figure 5-26). Alternatively, frictionless contact led to some improvements, but did not completely solve the expansion issues. 


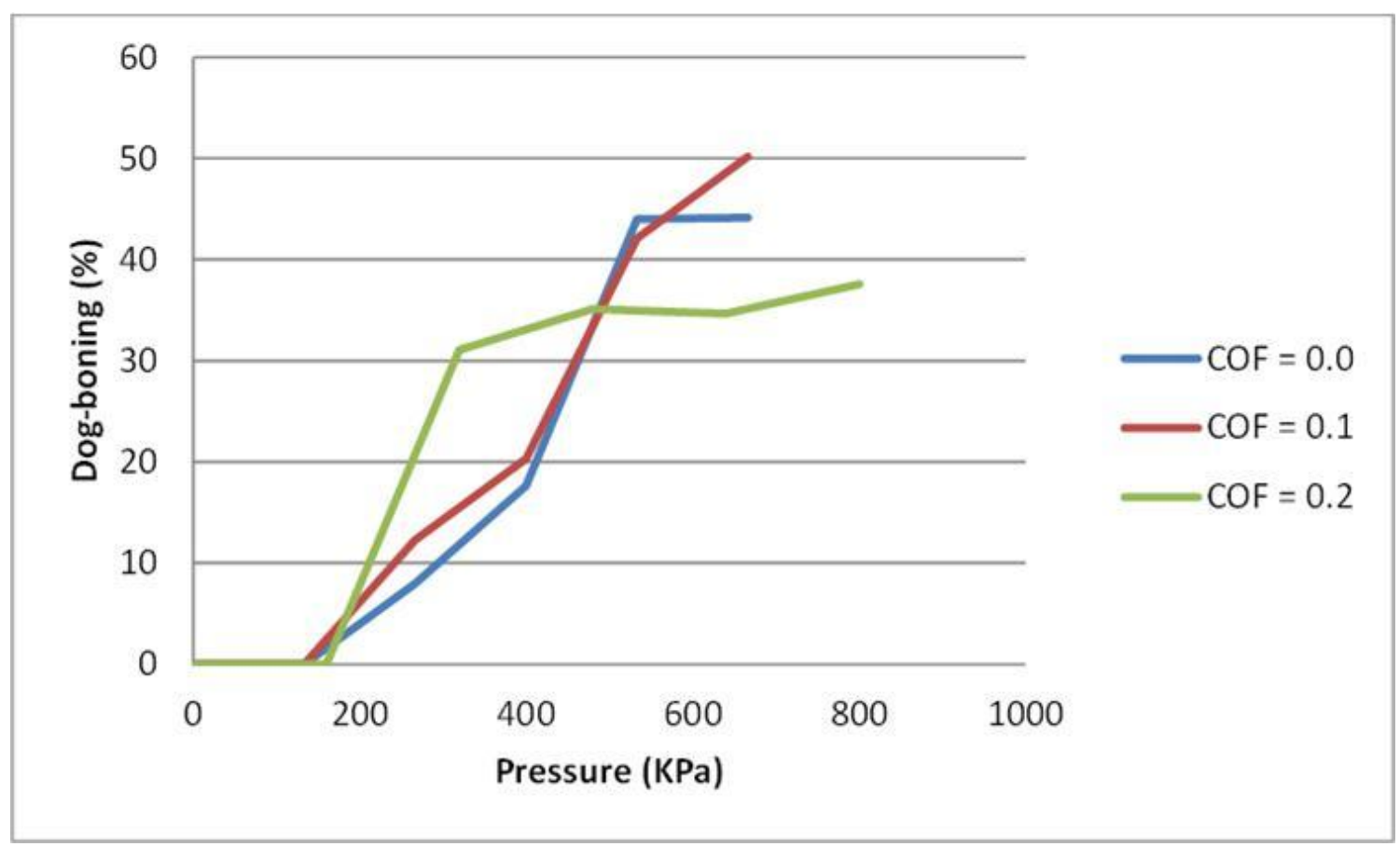

Figure 5-26 Coefficient of friction versus dog boning.

The total normal force required to expand the stent (Figure 5-28) was investigated for the uniform cylinder, balloon, and progressive balloon expansion methods. The diameter was constant along the length of the stent for the uniform expansion model, while the normal force varied over the length, demonstrating its highest value at the middle of the stent. This is expected and results from the uncoiling process. In the case of balloon expansion, the balloon pressure was constant (the average pressure across the five chambers in the progressive expansion study was used), and the diameter varied along the length of the stent. The normal force was estimated as the balloon pressure multiplied by the contact area of the stent. The stent diameter was measured at the central and distal coils, corresponding to maximum and minimum values, and the total normal force was plotted as a function of the stent diameter. 


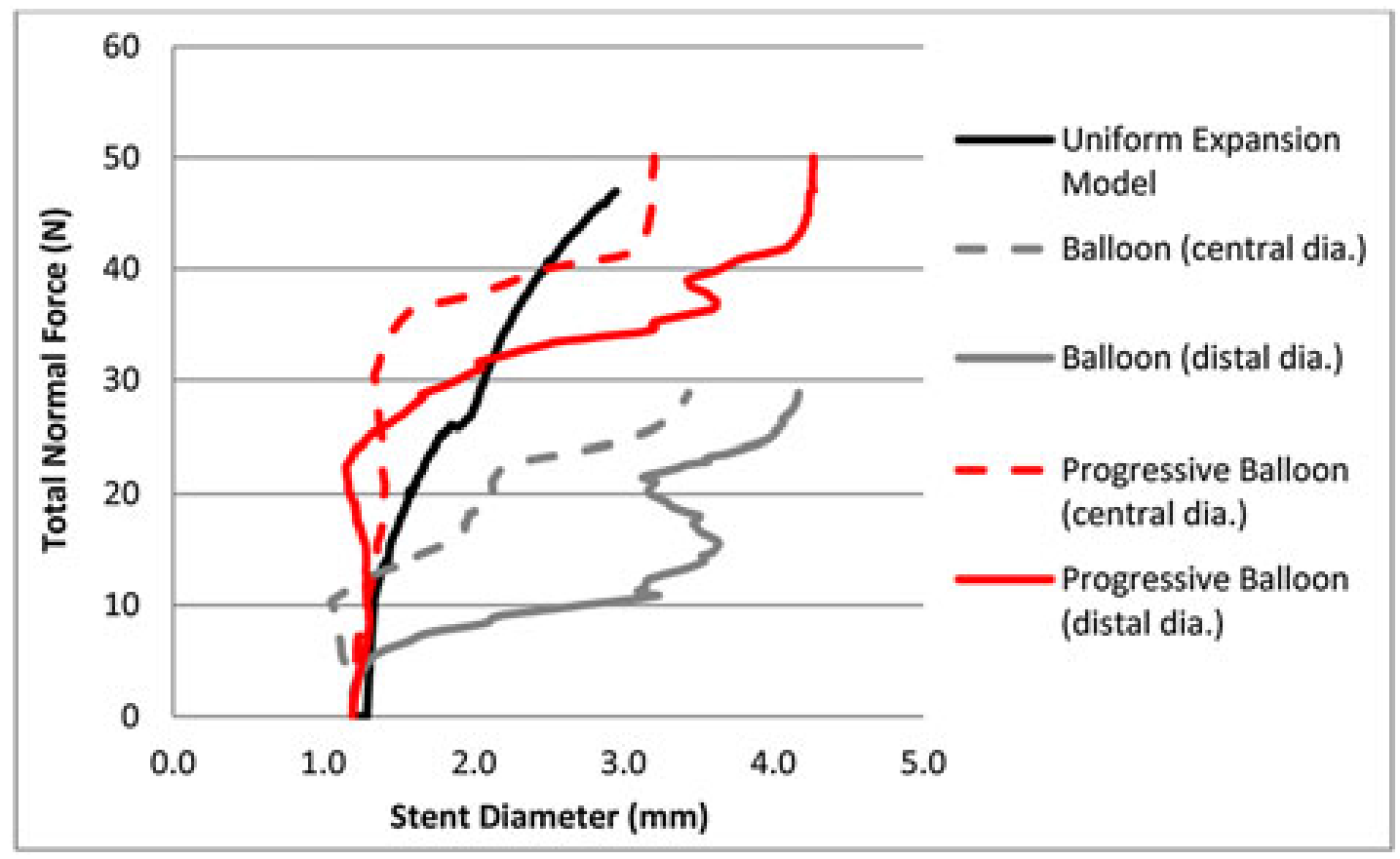

Figure 5-27 Expansion normal force versus stent diameter.

\subsection{Summary}

Although helical stent designs are ideal candidates for biodegradable polymeric stenting devices, their expansion is challenging due to non-uniform deformation (dog boning) and subsequent interaction (overlap) of the stent coils. Using a folded balloon expansion method, it was evident that available commercial balloons promote dog boning and interaction between helical stent coils during expansion, as noted in comparable experimental tests.

The investigation confirmed that balloon expansion is the only realistic method to model stent boundary conditions, and identified non-uniform expansion (dog boning) as an important issue to address for successful deployment of helical stent designs. The stent-folded balloon expansion method was used to investigate mitigation of non-uniform deformation for helical stent designs. Although modifications to the stent geometry, as well as the balloon's stiffness and geometry, provided small improvements, the inherent expansion mode and shape of the balloon still resulted in significant non-uniform expansion.

An approach to eliminate these issues was proposed using a progressive pressurization pattern that expanded the stent from the middle coils outwards. This method resulted in full expansion of the stent, and significantly reduced non-uniform deformation. In general, this non-uniform 
deformation is a shortcoming in the expansion of helical stent designs and future work will focus on a practical implementation for the proposed progressive expansion balloon approach, which could also benefit traditional stent designs that suffer from non-uniform expansion. This study has provided insights into an improved expansion method for helical stents, which may also be applicable to other stent designs to limit arterial wall injuries and potentially reduce the risk of late-restenosis. Upcoming chapters focus on the integration of an artery model with the stent expansion model. 


\section{Chapter 6 \\ Analysing helical stent deployment inside a human coronary artery with plaque buildup}

\subsection{Introduction}

Cardiac or coronary stents were originally developed to provide structural support to arteries that have been reopened or widened through angioplasty procedures. However, stent deployment and expansion may damage the arterial wall, leading to restenosis or re-narrowing of the blood vessel. Recent FEM studies [Gervaso, 2008] suggest high stress imposed upon the artery walls during stent expansion is the primary source of such injuries. However, it is important to note that stents behave differently when expanding inside an artery with plaque buildup than when expanding freely (without consideration of the surrounding artery). Therefore, the stent design process should incorporate analysis during free expansion, as was examined in Chapter 5, and also while expanding inside an artery with plaque buildup.

Numerical modeling, particularly finite element modeling, is useful for evaluating and optimizing stent design. Two numerical models have been developed to support evaluation of helical stent expansion inside an artery with plaque buildup, including:

- Angioplasty model, which describes folded balloon expansion inside arteries with and without plaque buildup

- $\quad$ Stenting model, which describes stent expansion using a folded balloon inside an artery with plaque buildup

The purpose of this chapter is:

- To evaluate the ability of a folded balloon to expand an artery with plaque buildup through analysis of results obtained from the angioplasty model.

- To evaluate the effects of the narrowed artery with plaque buildup on stent expansion using the stenting model, and then compare the results against those obtained from the freeexpansion model presented in Chapter 5. 
- To examine the effects of stent expansion on the artery wall and plaque buildup by comparing results obtained from the angioplasty and stenting models.

In this chapter the material models are integrated in a commercial Finite Element code (LS Dyna) and verified against experimental data. While the mechanical properties of arteries and plaque are important considerations in stent design, experimental characterization of these tissues falls outside the scope of this dissertation. Therefore, when necessary, information available within the published literature was used. For both the artery and plaque material models, sensitivity of the results to the mesh density was also examined with a thick wall cylinder under axial stretch. In this chapter the angioplasty and stenting models are developed and analyzed.

\subsection{Development and verification of the artery and plaque constitutive model}

Material constitutive models used in this study to describe the artery wall and plaque buildup are based on the experimental results of Yang et al [Yang, 2009] and Loree et al [Loree, 1994], respectively. The experimental results were implemented as a general hyperelastic material model available in LS DYNA (i.e., mat_77 [Christensen, 88]), and digitized as stretch-true stress measurements. The coefficients of the hyperelastic model were calculated using a regression method in the finite element program (Table 6-1). The code allows us to use 3,4 or 5 coefficients. Since we had to examine the material's behaviour up to the failure point, the 5coefficient option, with $\mathrm{R}^{2}$ close to 1 , was selected to capture the material's behaviour for the longest duration possible during the expected large deformation. With a higher number of coefficients, the fitted curve can capture all the curvatures of the material model stress-strain curve and will represent the material constitutive model closely.

For hyperelastic materials, the Cauchy stress $\sigma_{\mathrm{ij}}$ can be given in terms of Cauchy-Green tensor, $\mathrm{B}_{\mathrm{ij}}$, as follows:

$\sigma_{i j}=-p+2 \frac{\partial W}{\partial I_{1}} B_{i j}-2 \frac{\partial W}{\partial I_{2}} B_{i j}^{-1}$

Equation 6-1

where $\mathrm{W}$ is the strain energy function

$W=C_{10}\left(I_{1}-3\right)+C_{01}\left(I_{2}-3\right)+C_{11}\left(I_{1}-3\right) \cdot\left(I_{2}-3\right)+C_{20}\left(I_{1}-3\right)^{2}+C_{30}\left(I_{1}-3\right)^{3}$

Equation 6-2 
Cij are the constants, which are determined by fitting the equation to the experimental results, and $\mathrm{I}_{1}, \mathrm{I}_{2}$, and $\mathrm{I}_{3}$ are stretch invariants

$I_{1}=\left(\lambda_{1}\right)^{2}+\left(\lambda_{2}\right)^{2}+\left(\lambda_{3}\right)^{2}$

Equation 6-3

$\mathrm{I}_{2}=\lambda_{1} \lambda_{2}+\lambda_{1} \lambda_{2}+\lambda_{1} \lambda_{2}$

Equation 6-4

$\mathrm{I}_{3}=\lambda_{1}^{2} \lambda_{2}^{2} \lambda_{3}^{2}$

Equation 6-5

Where $\lambda \mathrm{i}(\mathrm{i}=1,2,3)$ are the principal stretches. As the material is incompressible $\mathrm{I}_{3}=1$

Table 6-1 Hyperelastic model constants and corresponding $\mathbf{R}^{2}$ value calculated for human artery [Yang, 2009] and plaque [Loree, 1994]

\begin{tabular}{|l|l|l|l|l|l|l|}
\hline $\begin{array}{l}\text { Hyperelastic } \\
\text { coefficients } \\
\text { (MPa) }\end{array}$ & $\mathrm{C}_{01}$ & $\mathrm{C}_{11}$ & $\mathrm{C}_{20}$ & $\mathrm{C}_{02}$ & $\mathrm{C}_{30}$ & $\mathrm{R}^{2}$ \\
\hline Artery & $0.3012 \mathrm{E}+00$ & $0.5018 \mathrm{E}+02$ & - & & & \\
& & & $0.2722 \mathrm{E}+02$ & $0.2210 \mathrm{E}+02$ & & \\
\hline Plaque & $\begin{array}{c}-0.7881 \mathrm{E}- \\
01\end{array}$ & $0.1361 \mathrm{E}+00$ & - & $0.1092 \mathrm{E}+00$ & $0.3384 \mathrm{E}+00$ & 0.9999 \\
& & $0.2119 \mathrm{E}+00$ & & & \\
\hline
\end{tabular}

The behaviour of the implemented materials was tested with a one element FE model. The element bore a similar formulation to that used in the final models (i.e., constant stress solid element) and had dimensions of $1 \mathrm{~mm}$ for each side. Loading and boundary conditions were implemented to produce a pure tension condition in the element. The material model behaved as expected (Figure 6-1). 


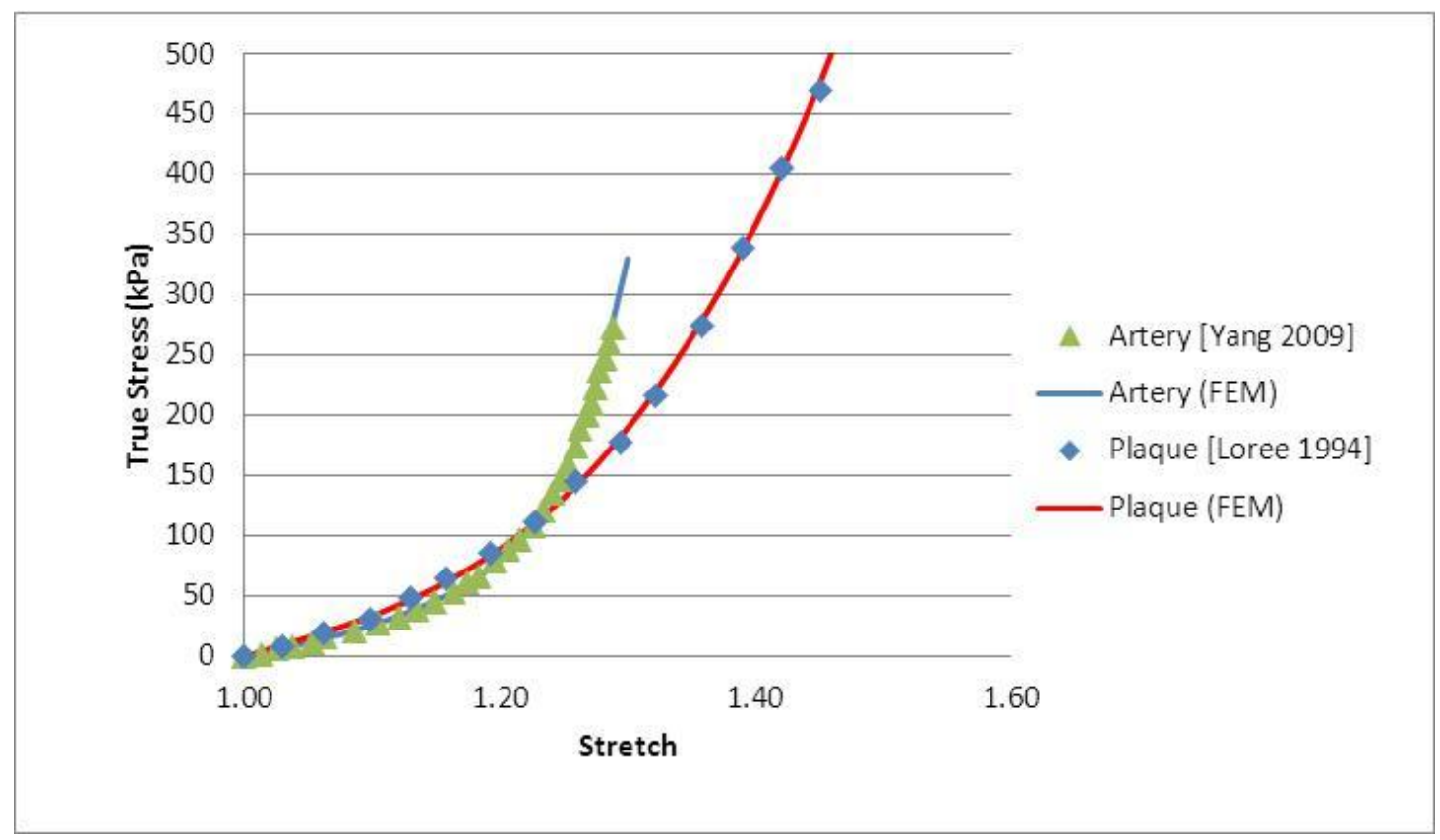

Figure 6-1 Hyperelastic material model verification based on experimental results for human LAD arteries from implementation of hyperelastic material models [Yang, 2009] and human cellular plaque buildup [Loree, 1994] and implemented in a one-element FE model.

The same one-element model was used to verify the material implementation for human cellular plaque buildup based on Loree et al [Loree, 1994] experimental results (Figure 6-1). The plaque is stiffer than the artery wall. Therefore, it is expected that at the same stretch value, the artery will reach the failure point before the plaque.

\subsection{Geometry}

Since FEM methods had not been used previously to evaluate stent expansion behaviour inside an artery, it was necessary to first analyze angioplasty balloon expansion inside an idealized cylindrical straight artery without plaque buildup. This strategy allowed us to examine the effect of expansion on the artery wall and evaluate the smoothness of load transfer between the balloon and arterial wall. In a subsequent study, the balloon was expanded inside an artery with a simple plaque buildup profile, allowing more rigorous analysis of an expansion scenario 
that includes a real artery with plaque topology. These steps revealed the effects of a boundary condition that mimics a real artery and plaque topology on stent deformation during expansion.

Since most cases of in-stent restenosis occur in the left anterior descending (LAD) artery and drug eluting stents (including the one used in this study) were designed to eliminate this condition, the present study focused on stenting scenarios in the left coronary arteries, specifically on the LAD artery. Although the geometries of human tissues vary, the inner diameter of the LAD artery is reported to be approximately $3 \mathrm{~mm}$ [Kang 1998; Holzapfel 2005], and its maximum length is reported to be $50 \mathrm{~mm}$ in humans [Claes, 2010]. Since stents and expanding balloons typically range in length from 8-12 mm, FEM studies often include arteries no longer than $20 \mathrm{~mm}$ to minimize the end effects on the stress distribution within the arterial wall [Karimi, 2013; Martin, 2013; Pant, 2012; Gervaso, 2008]. The present study took a similar approach, where the artery was assumed to be a straight cylinder with inner diameter of $3 \mathrm{~mm}$ [Pant 2012], and wall thickness of approximately $0.7 \mathrm{~mm}$ [Pant, 2012] and length of $20 \mathrm{~mm}$. The plaque model assumed a crescent shape geometry and thickness commonly used in FEM studies [Lally, 2005; Bressloff, 2013].

\subsection{Mesh convergence in artery and plaque}

A cylinder with wall thickness similar to that of an actual artery was used to examine mesh convergence in the artery model and the plaque model. An axial stretch was applied to the cylinder's wall to produce the 3D loading condition (Figure 6-2). The artery was modeled as a cylinder with a thickness of $0.7 \mathrm{~mm}$, inner diameter of $3.0 \mathrm{~mm}$, and a length of $20 \mathrm{~mm}$. As expected, axial stretch decreased the radius of the arterial wall and produced a 3D deformation in the elements. 


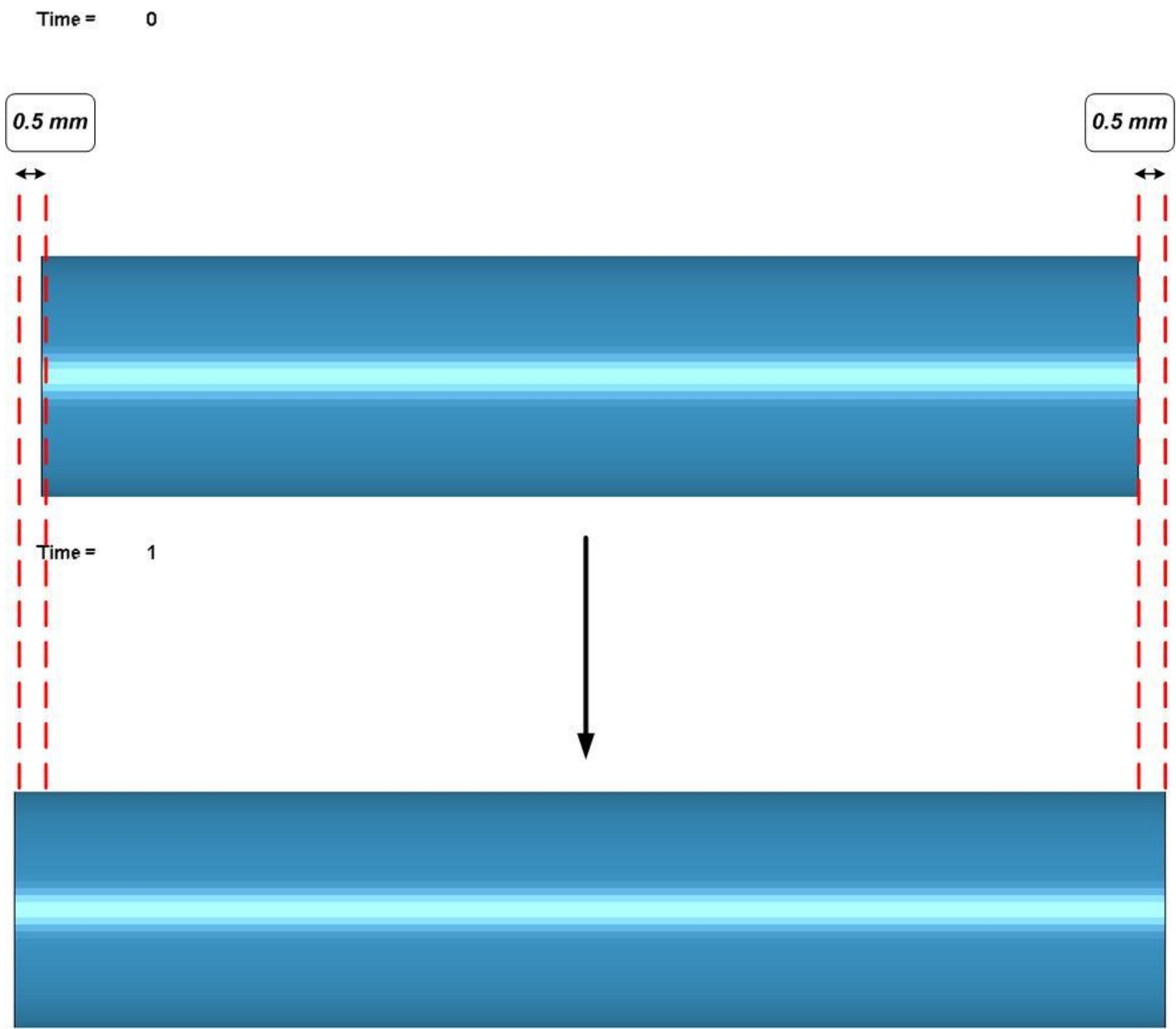

$$
r \stackrel{z}{x}_{x}
$$

Figure 6-2 Mesh convergence model: a cylinder under axial stretch loading

Mesh convergence in the artery and plaque were tested separately, as shown in Figures 6-3, 64, 6-5, and 6-6. The mesh dimension was gradually decreased by dividing the element dimensions in half. The von Mises stresses at the middle of artery wall during the axial stretch are reported. 


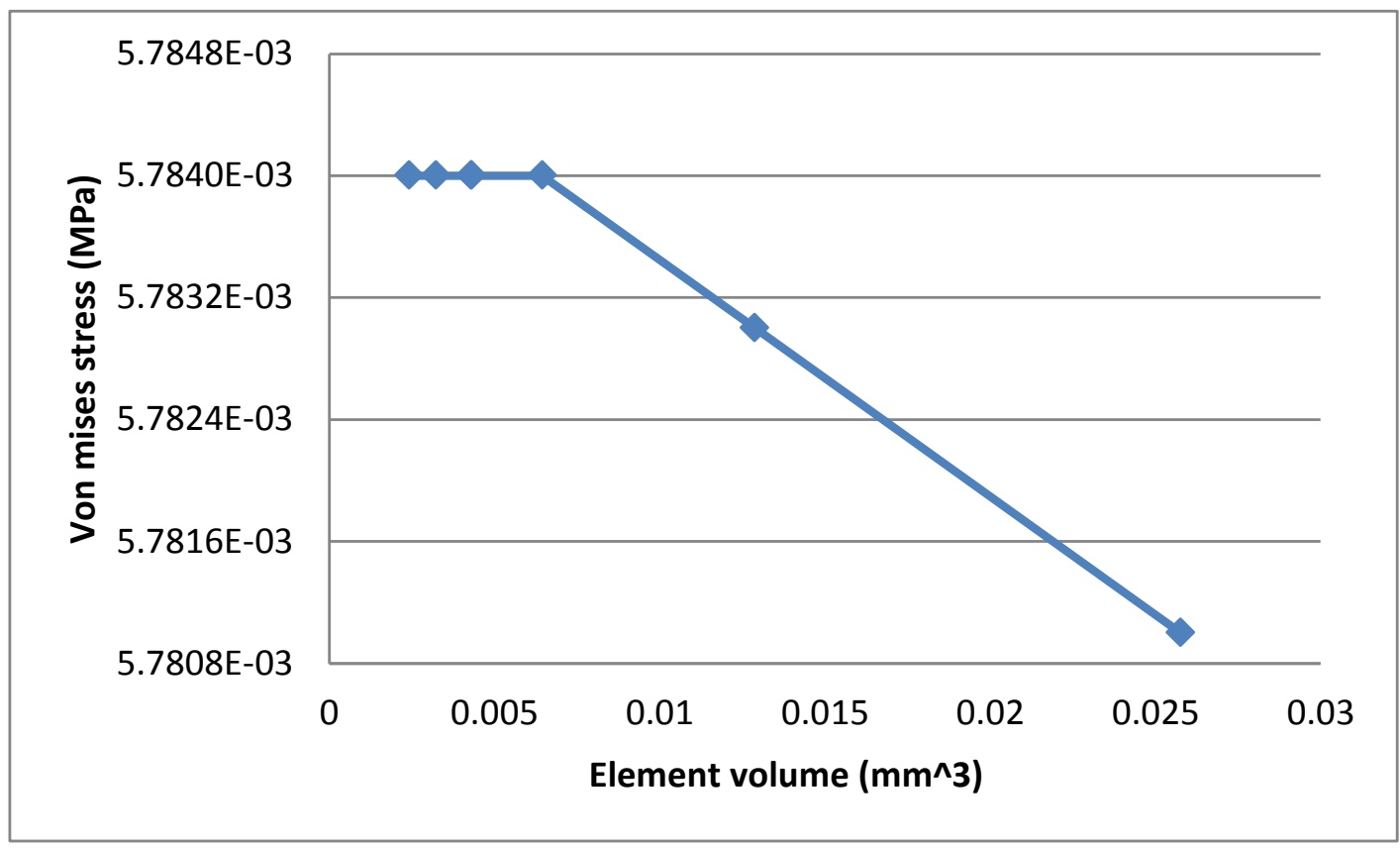

Figure 6-3 Mesh convergence for artery elements based on von Mises stress (MPa)

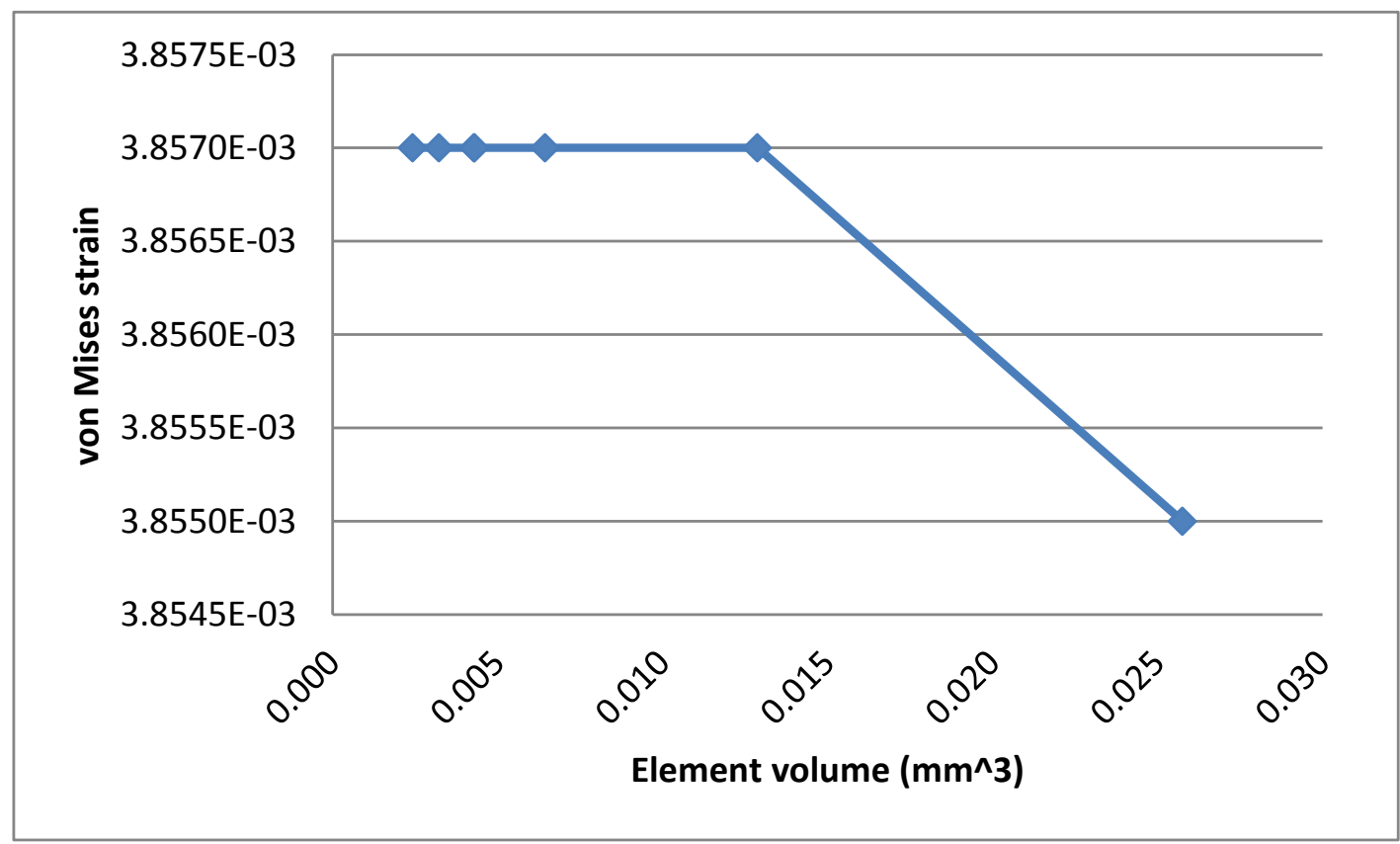

Figure 6-4 Mesh convergence for artery elements based on effective strain 


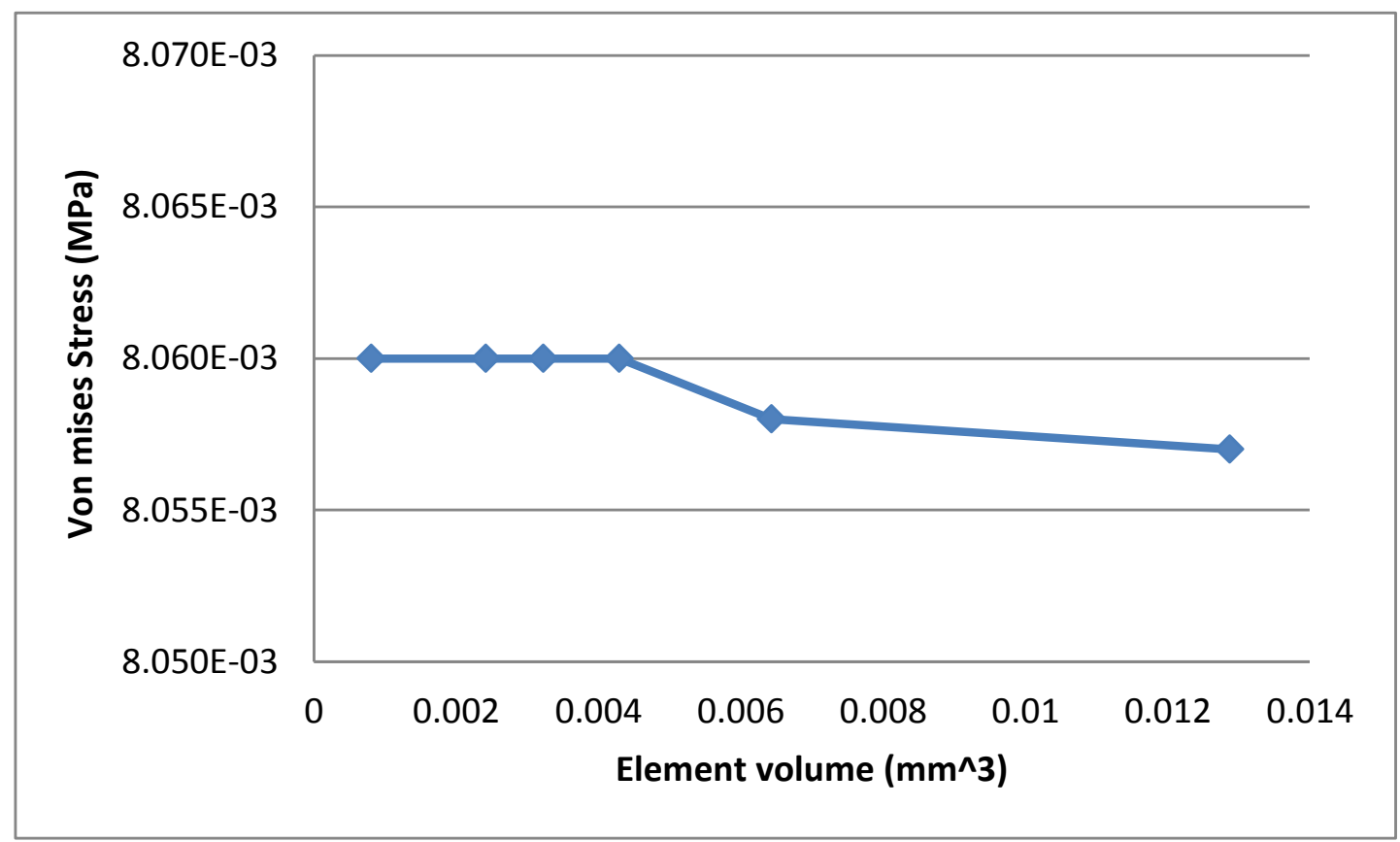

Figure 6-5 Mesh convergence for plaque elements based on von Mises stress (MPa)

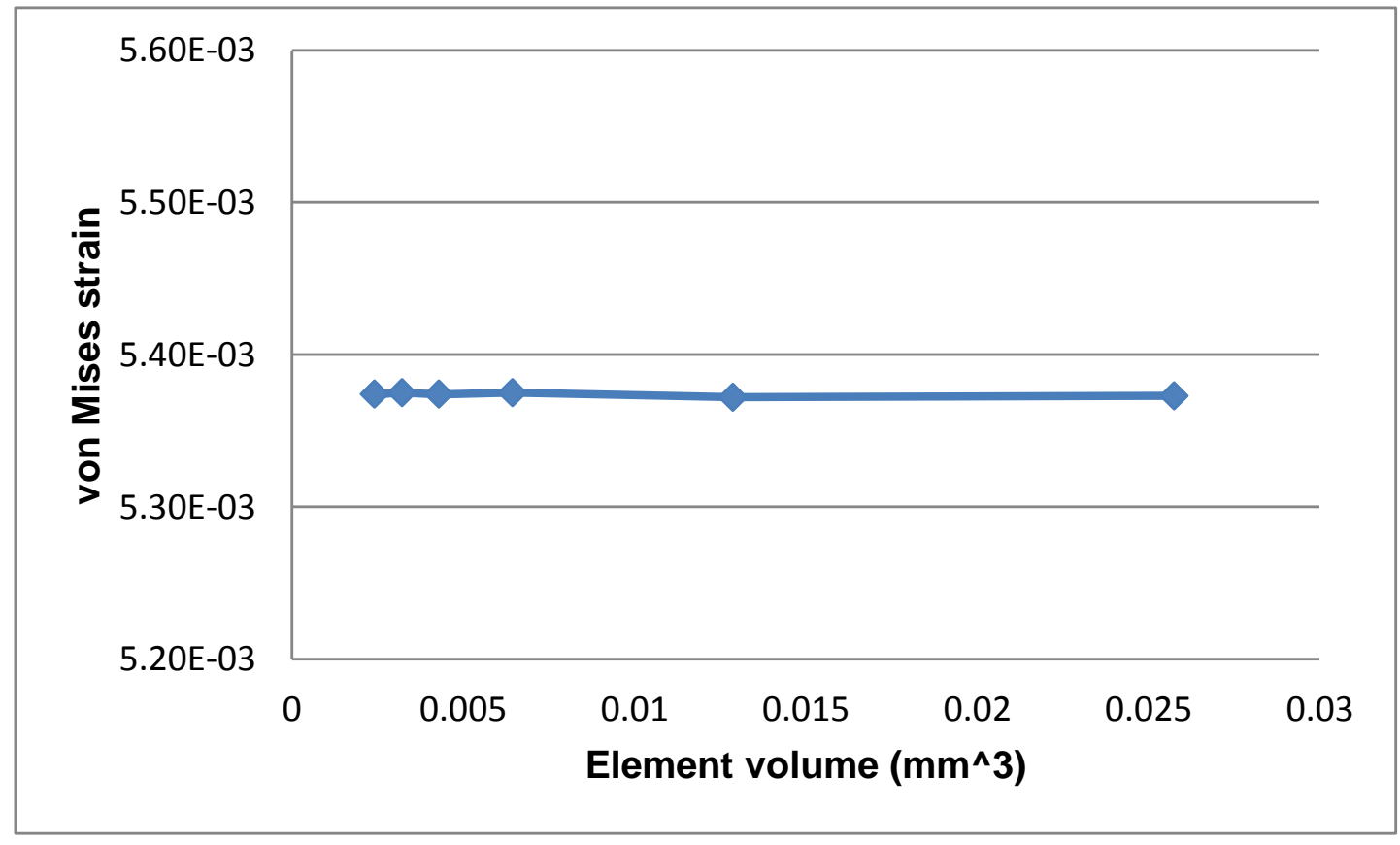

Figure 6-6 Mesh convergence for plaque elements based on effective strain 
Results show that the artery model converged in the element volume equal to $0.00644 \mathrm{~mm}^{3}$ (i.e., $0.276 \mathrm{~mm} * 0.2 \mathrm{~mm} * 0.116 \mathrm{~mm}$ in circumferential, axial and radial directions, respectively) and the plaque model converged in $0.0043 \mathrm{~mm}^{3}$ (i.e., $0.133 \mathrm{~mm} * 0.276 \mathrm{~mm} *$ $0.117 \mathrm{~mm}$ in circumferential, axial and radial directions, respectively). Since the artery and plaque are in close contact, the element density in the boundary was selected to have equal element length on the boundary surface. Therefore, similar densities should be selected for both artery and plaque. Consequently, an element density equal to or smaller than $0.0043 \mathrm{~mm}^{3}$ should be used for modeling. However, to avoid hourglassing error, a smaller value (i.e., $0.0024 \mathrm{~mm}^{3}$ which is $0.17 \mathrm{~mm} * 0.1 \mathrm{~mm} * 0.14 \mathrm{~mm}$ in circumferential, axial and radial directions, respectively) was used for both artery and plaque in the actual model. The artery was meshed with 200, 80 and 5 elements along the length, width and circumferential directions of the artery wall, respectively. Figure 6-7 shows the geometry of angioplasty model without plaque.

\subsection{Angioplasty model}

\subsubsection{Angioplasty model without plaque}

First, the balloon was expanded in a straight artery (cylinder) without any plaque buildup (Figure 6-7). The balloon and artery were $13.77 \mathrm{~mm}$ and $20.0 \mathrm{~mm}$ long, respectively. As previously described, the artery length was selected to ensure that the length did not affect the stress distribution of the artery area in contact with the balloon angioplasty and stenting area. The validity of such assumption will be examined later in this chapter.

Symmetry boundary conditions were applied at the two ends of the artery to provide continuity with the neighboring parts (the two ends were fixed to prevent axial displacement and radial and circumferential rotation). 

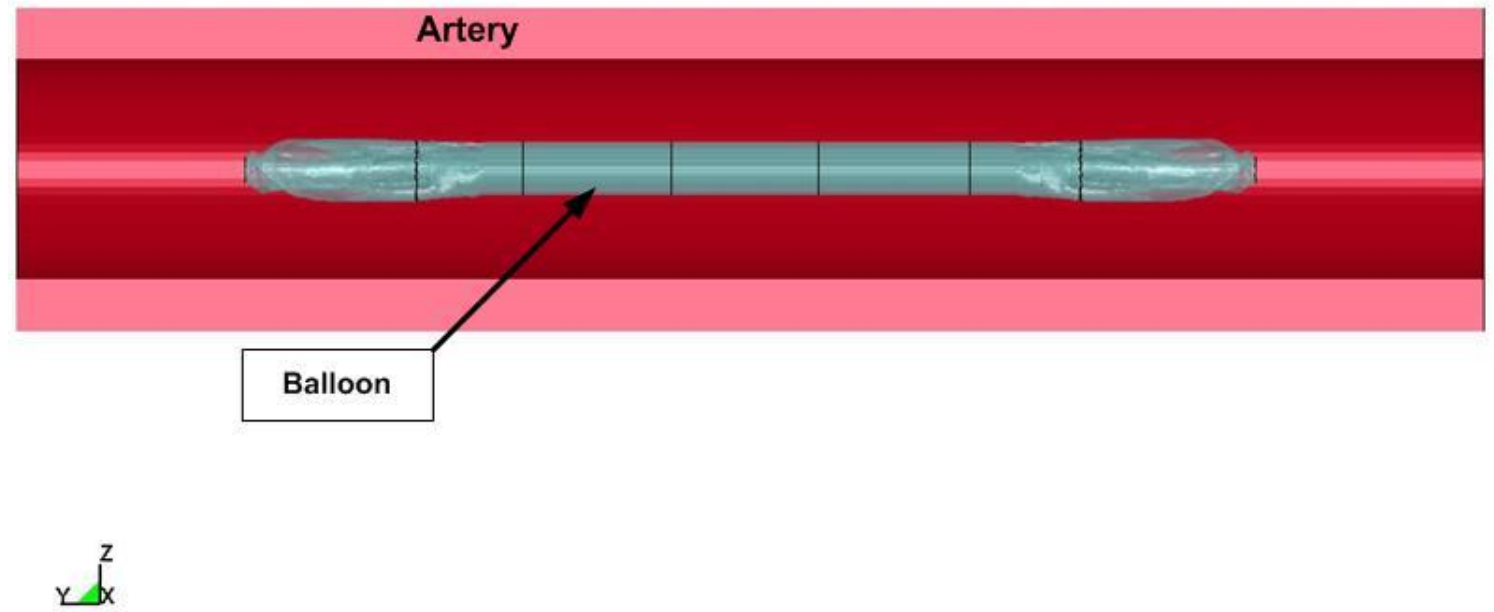

Figure 6-7 Geometry of the angioplasty model without plaque buildup

It is known that arteries shorten after being cut from the arterial tree [Holzapfel, 2005]. Therefore, when working, arteries are not in a stress-free state. The axial stretch in human coronary arteries is $5 \%$ [Holzapfel, 2005], which is equal to $1 \mathrm{~mm}$ axial stretch for a $20 \mathrm{~mm}$ artery. During the first 1 second of simulation, axial stretch was applied to the two ends of the artery, stretching each end $0.5 \mathrm{~mm}$, to mimic an artery operating under normal conditions. Figure 6-8 shows the von Mises stress distribution on the artery wall following axial stretch after the stress has reached a steady state. The stress distribution is uniform considering six significant figures.

Blood pressure is normally in the range of $16-10 \mathrm{kPa}$, which is 2 to $1.25 \%$ of the expected maximum pressure required to expand the stent $(800 \mathrm{kPa})$. On the other hand, the axial stretch in each end of the artery is $0.5 \mathrm{~mm}$, which is $30 \%$ of the maximum radial expansion in the artery (which is $1.5 \mathrm{~mm}$ ). Therefore, the blood pressure can be neglected but the axial stretch is not negligible. 


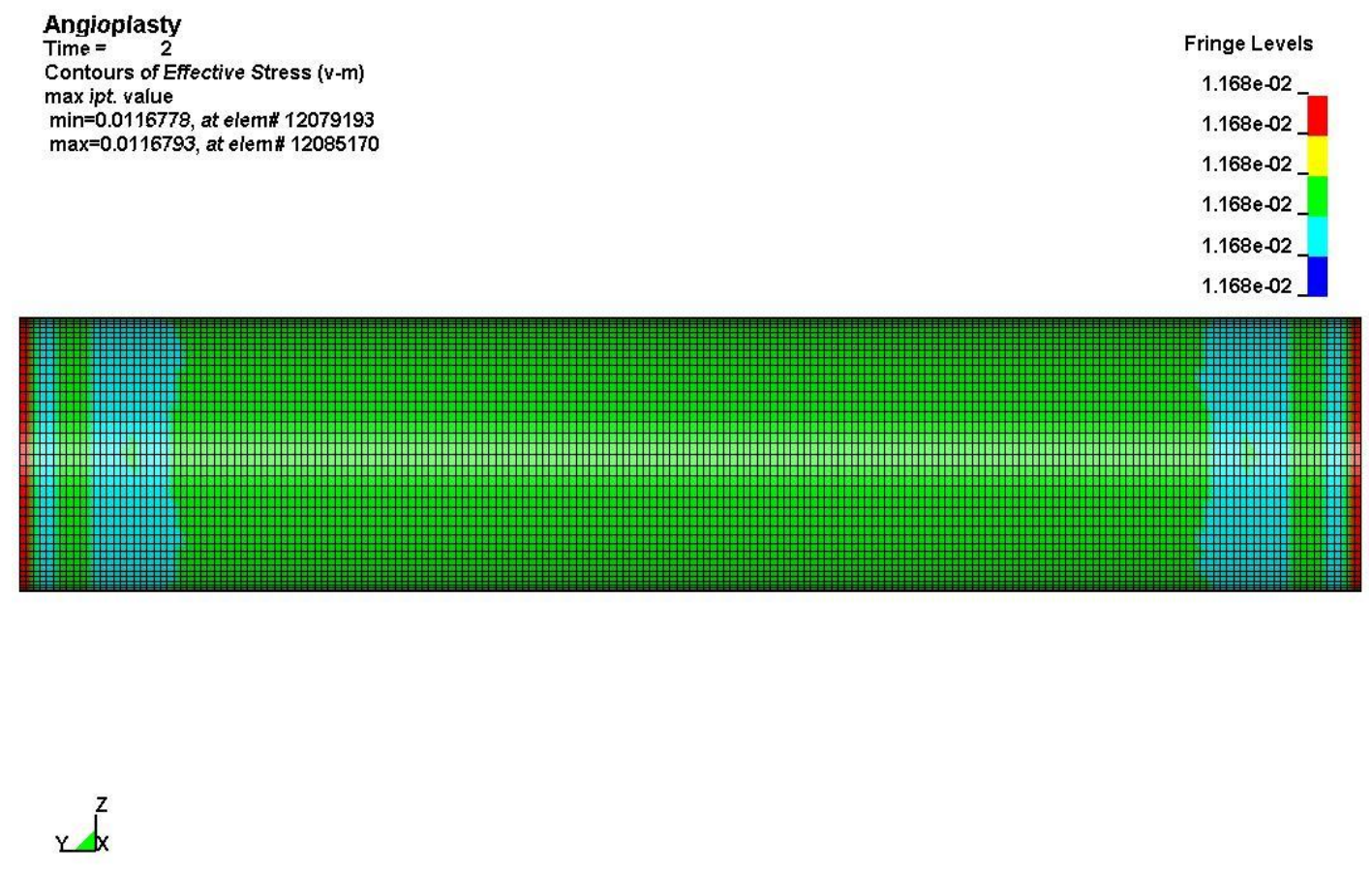

Figure 6-8 von Mises stress distribution (MPa) after axial stretch

During expansion, pressure was initially applied to the middle segment of the balloon and then progressively outward (distally) towards the end caps (for a detailed description of pressure application, see Chapter 5.3). The final and highest pressure (800 KPa) was reached in 20 seconds. We employed the same progressive pressurization pattern here as was applied in the stent expansion model in Chapter 5 to enable direct comparison of the final stress distributions in the artery after angioplasty and stenting.

During expansion, the three folds of the balloon unfold and then expand. At the end of expansion the balloon comes into contact with the artery wall. As described in Section 4.6.1, the initial balloon configuration includes three folds and is wrapped around a central wire Points along the artery where it contacts the folded edges of the expanded balloon experience a small local over-expansion of $0.04 \mathrm{~mm}$ (Figure 6-9). Since there are three folds, there will be six overexpansion points on the balloon that show themselves as stress concentration areas on the artery wall. Since the balloon had minimum contact with the artery in vicinity of the plaque buildup zone, the slight over-expansions did not affect the overall stress distribution. These stress 
concentration areas are not a factor during angioplasty or stenting procedures carried out in arteries with plaque buildup, since the level of stress concentrations at the artery and plaque are much higher than the balloon overstretch points.

\section{Angioplasty
Time $=21$}
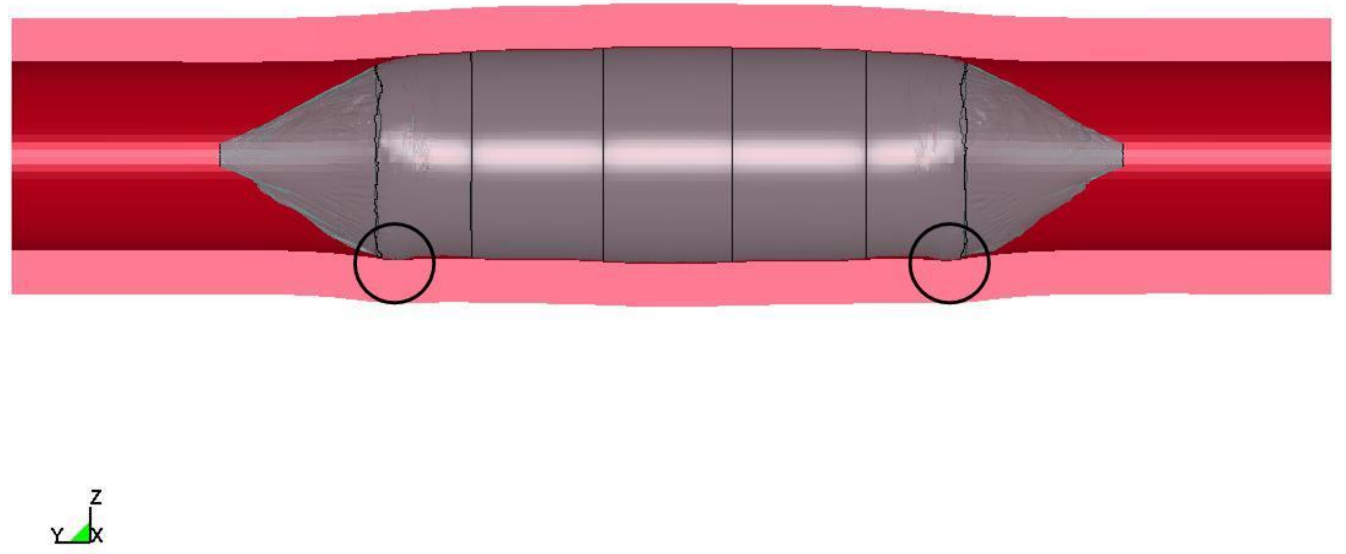

Figure 6-9 Expanded geometry of the angioplasty model without plaque buildup. The two overstretched areas are marked with circles.

The balloon was expanded to an outer diameter of $3.28 \mathrm{~mm}$, which then expanded the inner diameter of the artery by $0.28 \mathrm{~mm}$ in the middle. The average stress distribution after maximum balloon expansion was $65 \mathrm{kPa}$ (Figure 6-1), which is not close to the failure point of the artery wall $(300 \mathrm{kPa})$. The stress distribution is located at the over-stretched point of the balloon. 


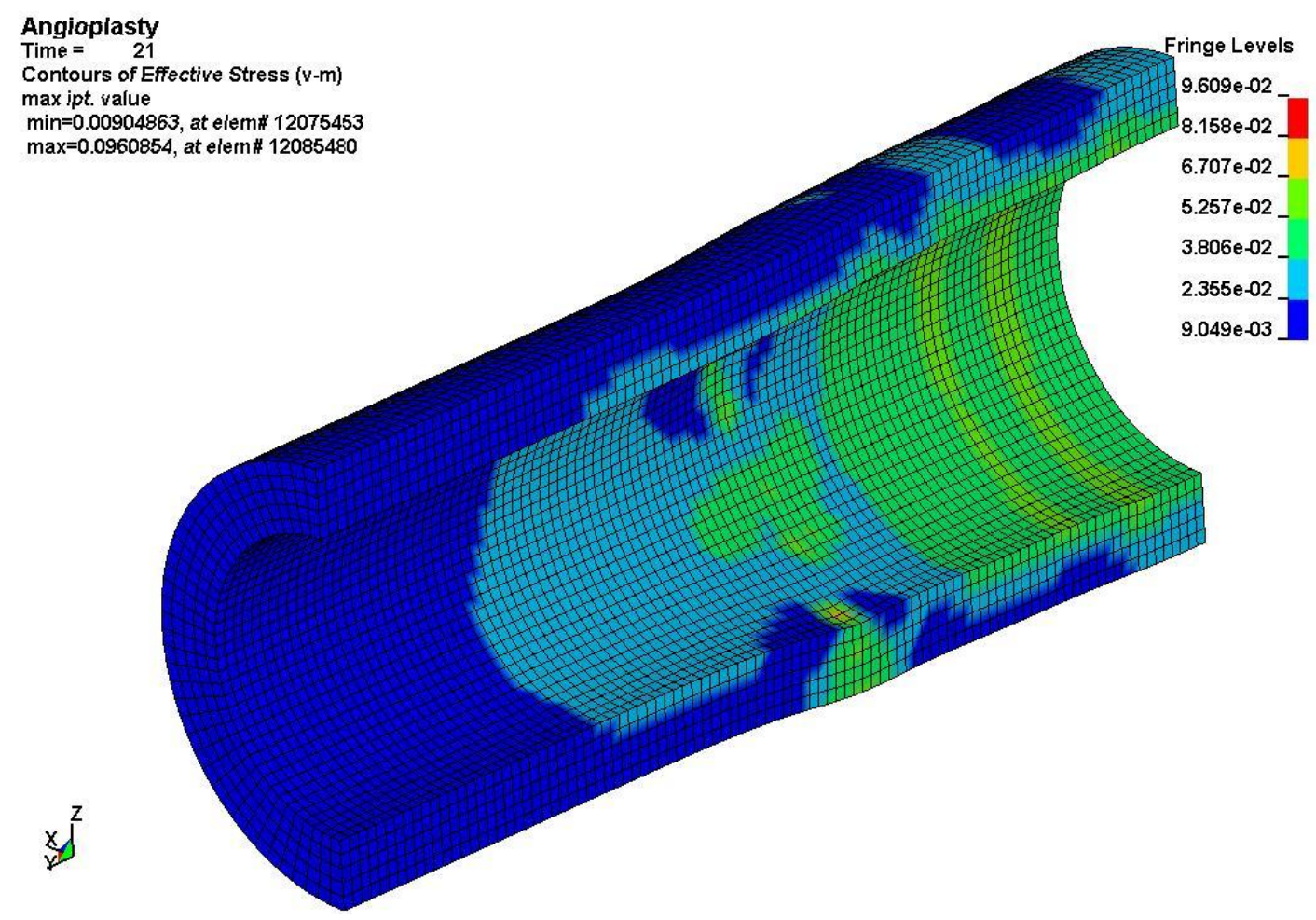

Figure 6-10 von Mises stress distribution (MPa) in the arterial wall during full balloon expansion (note that due to the model's symmetry, only half of the artery is shown)

\subsubsection{Angioplasty model with plaque}

By adding plaque to the previous angioplasty model we were able to more rigorously assess balloon expansion under more realistic intervention conditions. Generally, stenting procedures are performed on arteries diagnosed to be beyond "significant conditions," which is defined as $50 \%$ stenosis [ACCF/AHA/SCAI Guideline, 2011] where the diameter of the affected portion of the artery is $50 \%$ of its normal diameter. Since the inner radius of the artery was $1.5 \mathrm{~mm}$, we applied a plaque thickness of $0.75 \mathrm{~mm}$ to create a significant artery condition. The artery and plaque have the same material density.

The element size of the artery and plaque at the artery-plaque surface were equal; therefore, the nodes were tied together and the force transfer between the surfaces was straight through the surface nodes of the artery and plaque (Figure 6-11). By defining the material interface in this way, we were able to save significant computation time. 


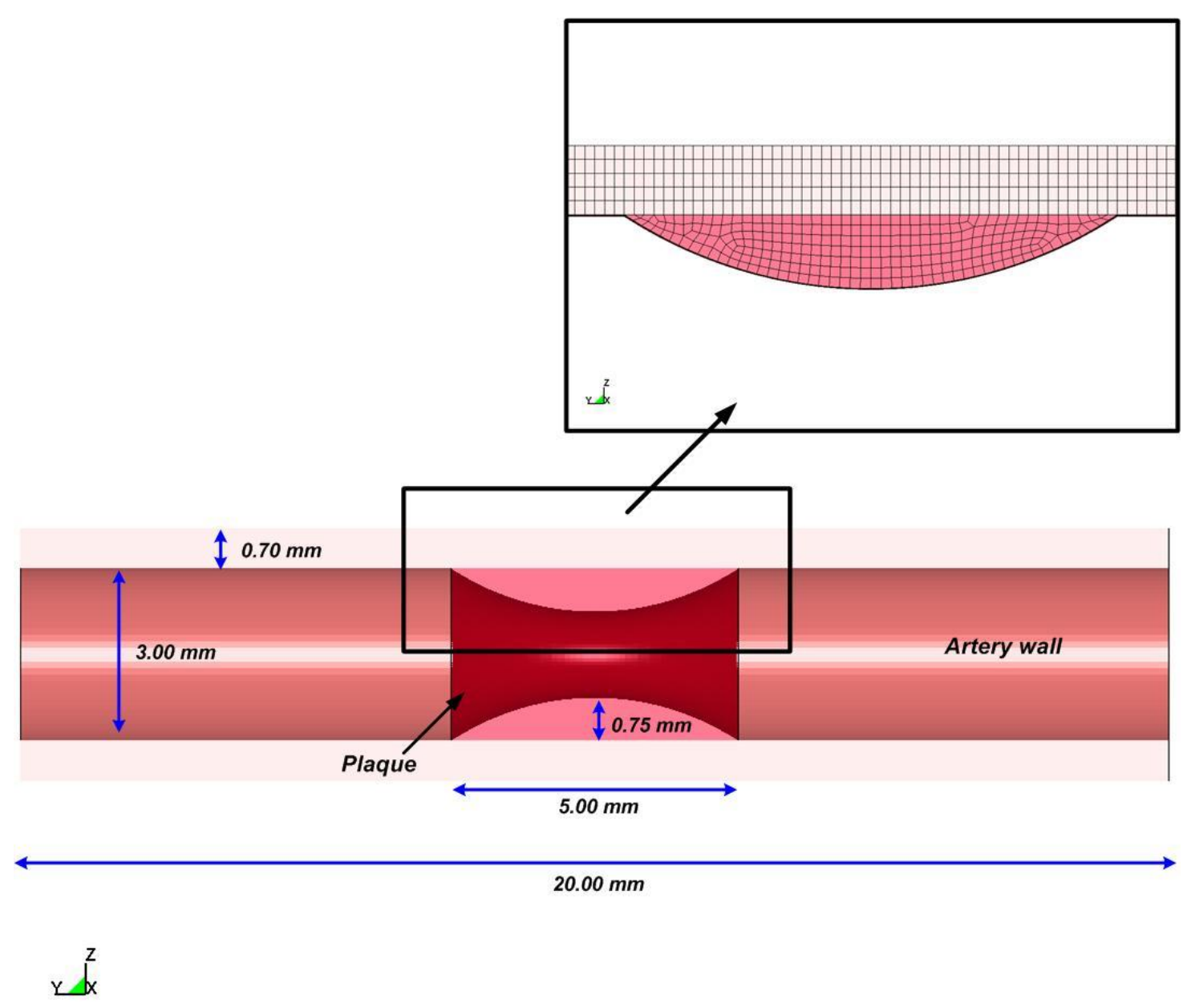

Figure 6-11 Mesh densities and the geometry of artery and plaque parts used in the angioplasty and stenting models

To examine the effect of axial stretch, two sets of angioplasty models were developed, i.e., angioplasty with and without axial stretch. In the first model, an axial stretch of $1 \mathrm{~mm}$ was applied to the ends of the artery and, because the surfaces of the plaque and artery were fused, to the plaque as well. The second angioplasty model did not involve an axial stretch; therefore, its loading time was $1 \mathrm{~s}$ shorter.

Although the stretch was applied in the axial direction, the artery was free to move in the radial and circumferential directions. Due to symmetry, no movement was observed in the circumferential directions. However, the artery and plaque were squeezed in the radial direction. Since the plaque component of the model is thicker and stiffer than the artery component, the 
radial displacement was smaller in that area. Therefore, the artery's outer surface became uneven with less radial displacement in the middle segment (Figure 6-12). Although the extent of radial displacement depends on the length of the artery, in general it is much smaller during axial stretch (maximum $0.006 \mathrm{~mm}$ ) than during angioplasty and stenting (maximum of $1.5 \mathrm{~mm}$ radial displacement is expected during angioplasty and stenting). Therefore, the role of artery length in shaping stress distributions is negligible.

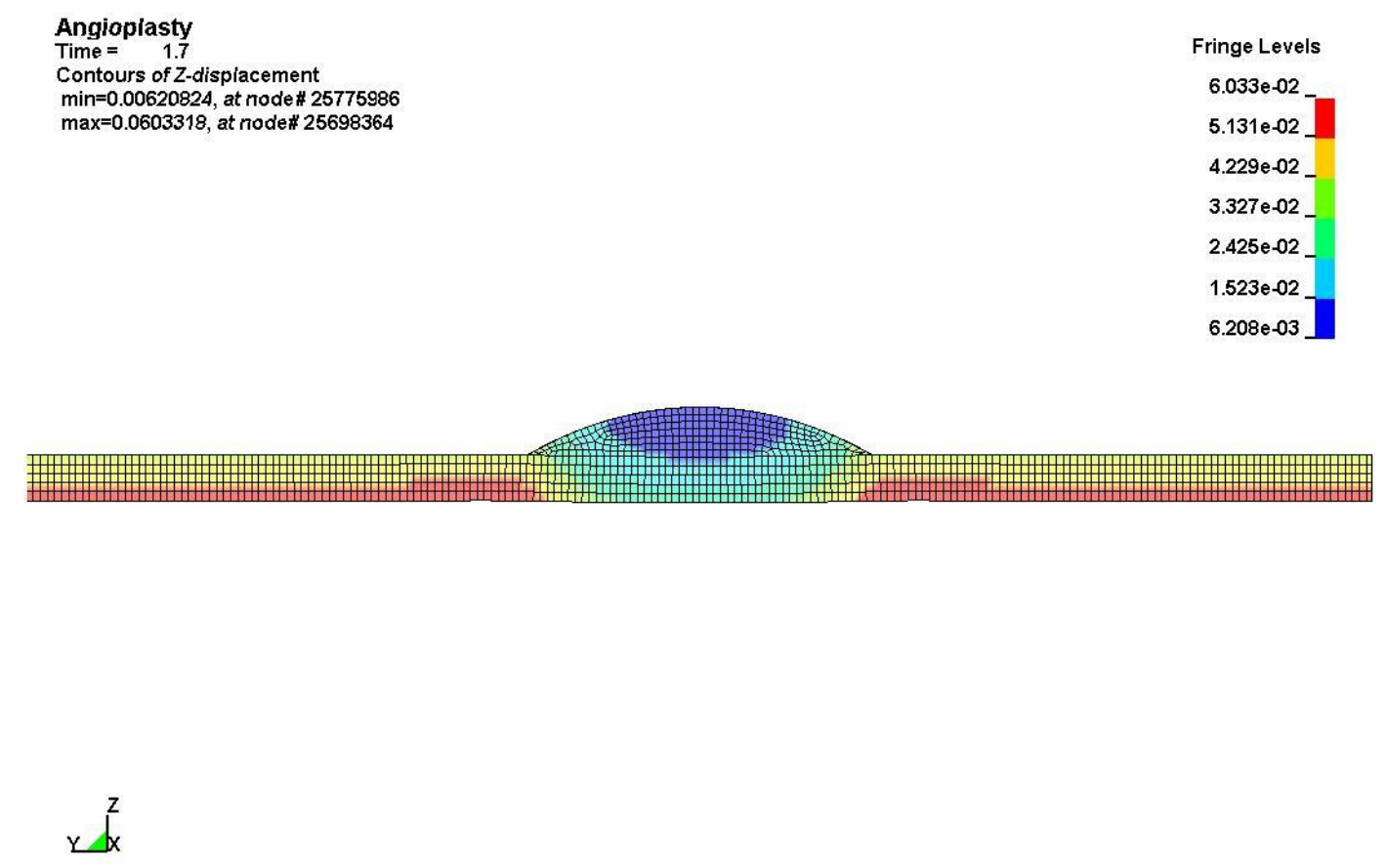

Figure 6-12 Radial displacement $(\mathrm{mm})$ in the artery and plaque elements after axial stretch

The von Mises stress distribution in the artery and plaque after axial stretch is symmetrical in the circumferential and axial directions (Figure 6-13). The uneven radial displacement along the artery causes a bending momentum on the artery at the vicinity of the plaque, putting stress on the artery wall. The effects are less than $10 \%$.

After axial stretch, which takes 1 second, balloon expansion using the progressive pressurization method begins. During the expansion stage, it takes more than 1 second for the balloon to touch the artery, providing the artery wall enough time to stabilize from the axial stretch. 
Under the progressive pressurization pattern (explained in Chapter 5), the middle segment of the balloon expands first. The expanded part touches the surface of the plaque and through this layer the force is transmitted to the artery wall (Figure 6-13). Expansion of the middle part of the artery applies a bending moment on the vicinity of the plaque. Note that this area was already under tensile stress due to the axial stretch (as explained above). As expected, the stress concentration is at the stent-plaque contact area.

Following expansion of the middle section of the balloon, pressure is applied to the more distal segments, gradually expanding them until they touch the surface of the artery. When the balloon is fully expanded, it successfully dilates the artery to reach its $3 \mathrm{~mm}$ inner diameter target (Figure 6-13). Throughout the expansion process, the highest pressure is experienced where the balloon presses upon the plaque deposit; therefore, the stress concentration remains on the plaque area and the part of the artery directly under the plaque (Figure 6-13). This means the section of the artery wall most prone to injury is located under the plaque buildup, a phenomenon that will be discussed in more detail later in the chapter. By comparison, in conventional balloon expansion systems, the entire balloon expands at once. Therefore, as the plaque is squeezed by the balloon, the healthy part of the artery is also severely stressed and could become injured. This highlights one of the main advantages of the proposed progressive pressurization pattern over conventional uniform expansion methods, even for angioplasty procedures that do not include stenting. That being said, proper positioning of the balloon is extremely important when using the proposed method. 

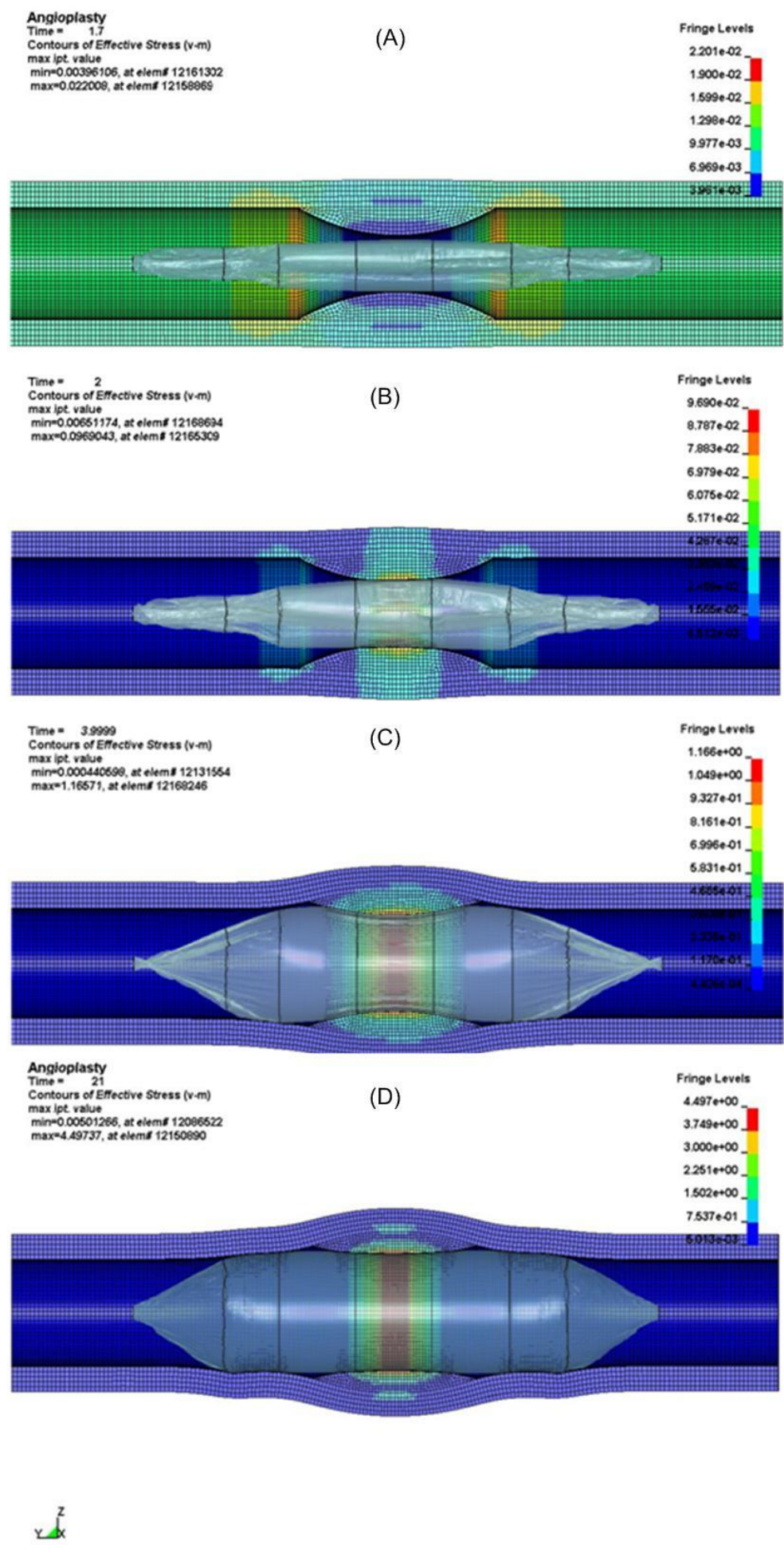

Figure 6-13 von Mises stress distribution (MPa) in the artery and plaque wall at the end of balloon expansion in the angioplasty model with axial stretch of $1 \mathbf{~ m m}$ 
Since applying axial stretch increases the computational cost of modeling, to test the effect of the axial stretch on the overall stress distribution during angioplasty, an angioplasty model without axial stretch was examined. The resulting von Mises stress distribution is shown in Figure 6-14.

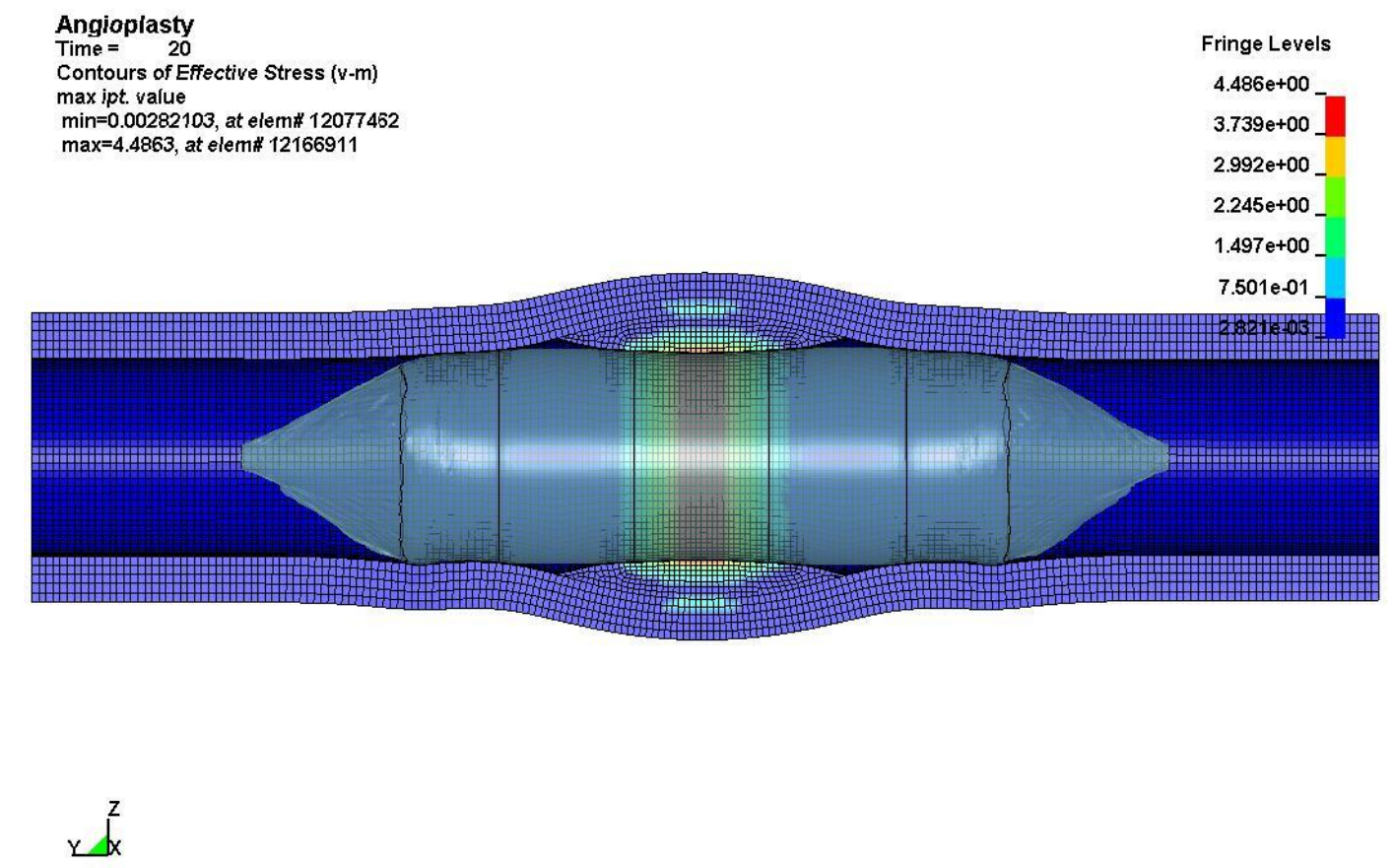

Figure 6-14 von Mises stress distribution (MPa) in the artery and plaque wall at the end of balloon expansion in the angioplasty model without axial stretch

The von Mises stress in the stress concentration area of the model that includes axial stretch is higher than that of the model without axial stretch by 3\%; therefore, axial stretch is considered for the stenting model.

Even when the balloon is fully expanded, the stress level near the ends of the artery remains at zero, indicating that the artery was long enough to diminish the potential effects of expansion on the artery ends and vice versa (as discussed in Section 6.6). 


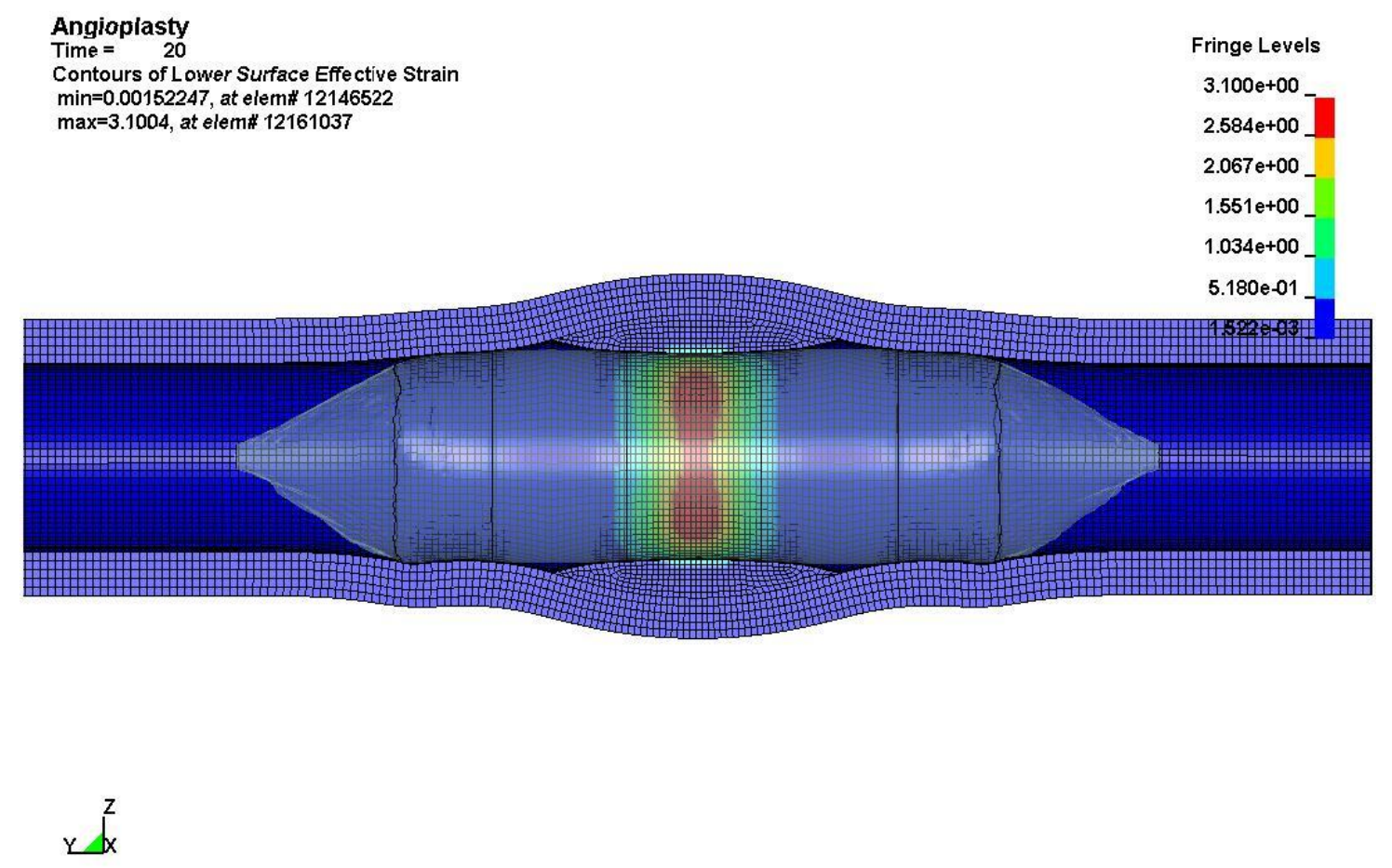

Figure 6-15 Effective strain distribution in the artery and plaque wall at the end of balloon expansion in the angioplasty model without axial stretch

The effective strains in the artery with and without axial stretch are very similar. The extent of the strained area is slightly larger in the artery without axial stretch (Figure 6-15) than in the one with axial stretch (Figure 6-14).

During expansion, the diameter of the artery directly under the plaque deposit grew wider than the adjacent healthy sections. Although the diameter of the artery and plaque in the model with axial stretch decreases after stretch, it expands to the same diameter of the artery without axial stretch (Figure 6-16). The diameter stretch of the arterial wall in the plaque area pulls the distal, healthy segments of the artery proximally towards the plaque, causing a radial decrease in this vicinity, which remains in contact with the balloon and under expansion. 


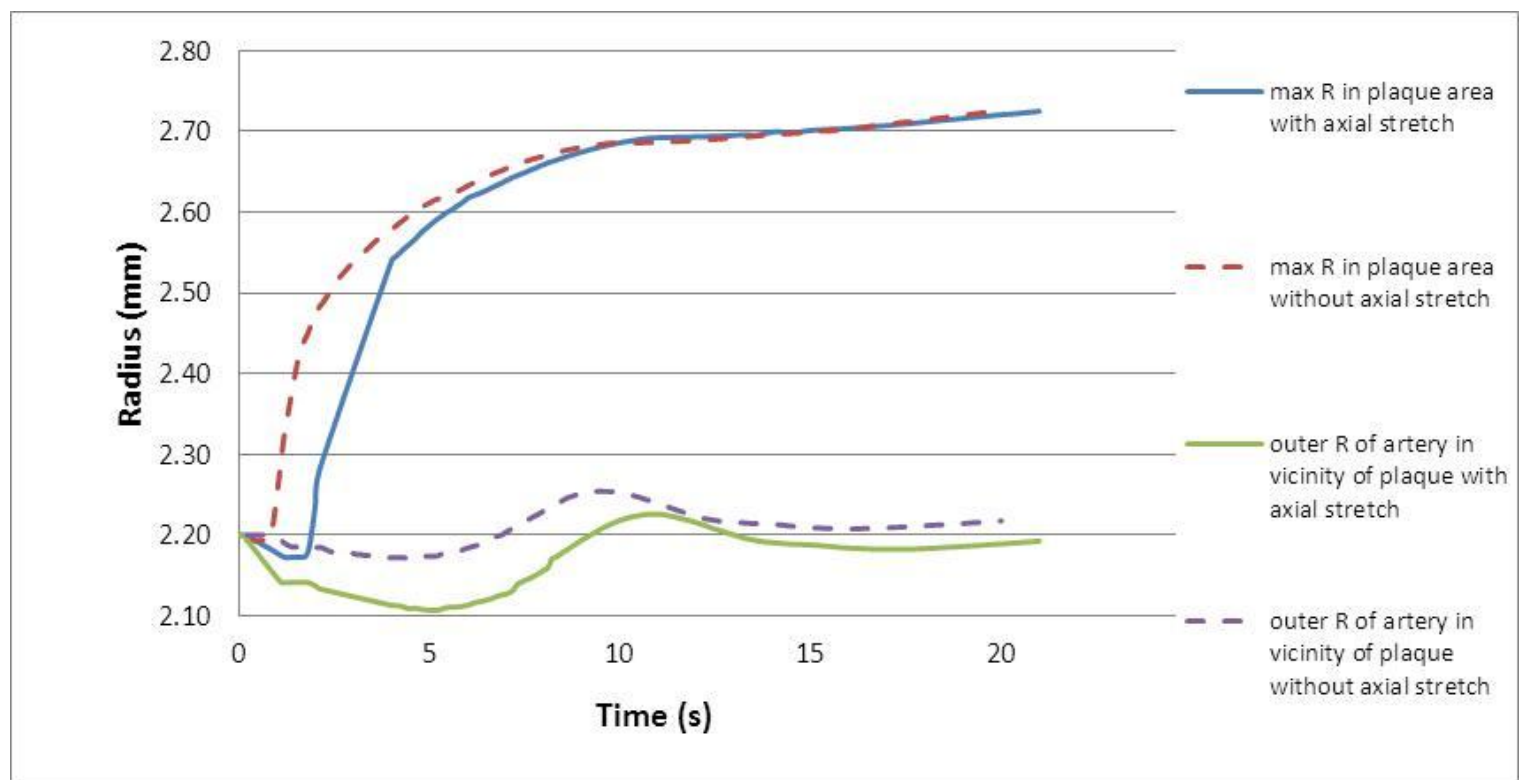

Figure 6-16 Maximum outer radius of the artery during expansion in the angioplasty model with and without axial stretch

\subsection{FE model of helical stent expansion inside an artery}

\subsubsection{Geometry and boundary conditions}

In this study, we applied the same modeling strategy for the artery as was applied in Section 6.5 (Figure 6-17). 

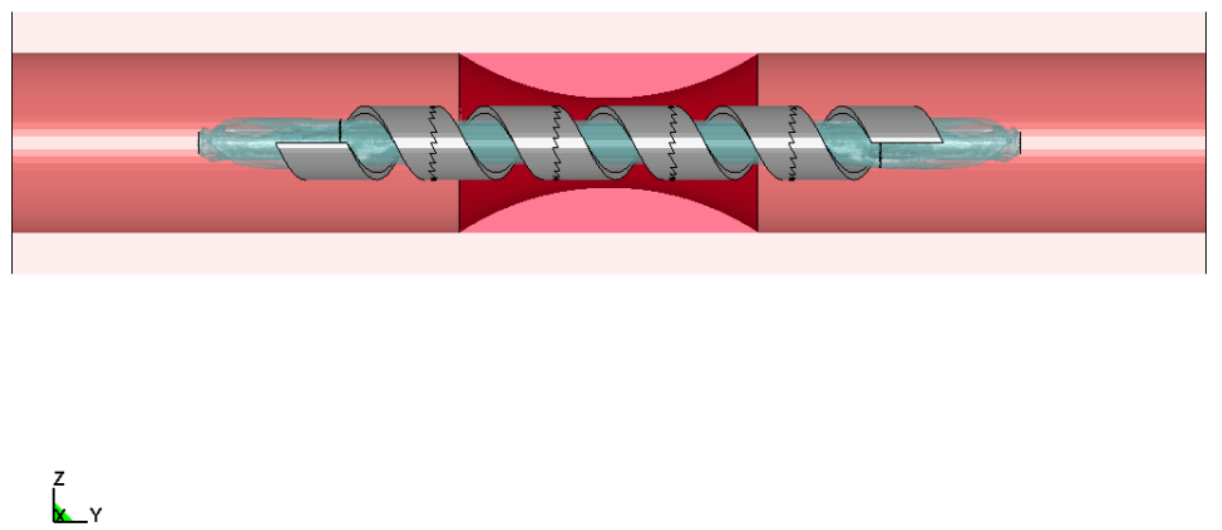

Figure 6-17 Geometry of the stenting model

The two ends of the artery were fixed in the axial direction and rotation in the $\mathrm{x}$ and $\mathrm{z}$ directions. Therefore, the artery was free to expand in the radial direction. This boundary condition resembles that of the wall of a section which has been cut from a long artery.

To define the interaction between the artery and the expanding stent, in addition to the contact interfaces among the balloon folds and between the stent and the folded balloon, frictionless automatic surface-to-surface contacts between stent and the artery as well as balloon and artery were considered. Since the initial contact between the stent and artery as well as between the balloon and artery happens toward the end of the simulation, the contact definitions were activated during the last $20 \%$ of the simulation time to reduce computation time and model run time. In addition to the contacts between the stent coils and the balloon, a frictionless surface-tosurface contact was applied between the artery's inner wall and the outer surface of stent. Since PLLA is hydrophobic [Fisher, 2007] and the stent-artery contact happens in a moist environment, it is valid to assume the stent-artery contact is frictionless. 


\subsubsection{Numerical considerations}

The artery was meshed with 80,000 fully integrated quadratic 8 node elements with element rotation (5 elements through thickness). The helical PLLA stent (5 coils, $1 \mathrm{~mm}$ width, $2 \mathrm{~mm}$ pitch, $0.12 \mathrm{~mm}$ thickness) was developed for this study as discussed in Section 4.4. The stent was meshed with 6,600 hexahedral elements and the mechanical properties of the PLLA were measured in vitro $\left(37^{\circ} \mathrm{C}\right.$ water) using an immersed dynamic mechanical analyzer (DMA). The stent was then implemented in the model using an elastic-plastic constitutive model with a von Mises yield criterion and an elastic modulus of $1.4 \mathrm{GPa}$, Poisson's ratio of 0.3 , and yield stress of $260 \mathrm{MPa}$.

The final model took 143 hours to run (in LS Dyna double precision version mpp R6.1.1) using a 128-node cluster. The model was forced to run with time steps equal to 1 e-5.

Signs of hourglassing were evident on the plaque surface. However, after comparing the level of hourglass energy in the plaque and artery against the internal energy, hourglass energy was found to represent less than $10 \%$ of the total energy and its effect on strain distribution of the model could therefore be ignored. Figure 6-18 shows the ratio of hourglass energy to total and internal energy during stent expansion inside an artery with plaque buildup. In the first 1.8 s, i.e., the initial $4.5 \%$ of the loading time, the ratio of hourglass energy to internal energy is over $5 \%$. We know from previous reports [Belytschko, 1984] that the ratio of hourglass energy to internal energy must be less than 10 to $15 \%$ to ensure valid and reliable results. Therefore, the ratio obtained in our study was considered satisfactory. 


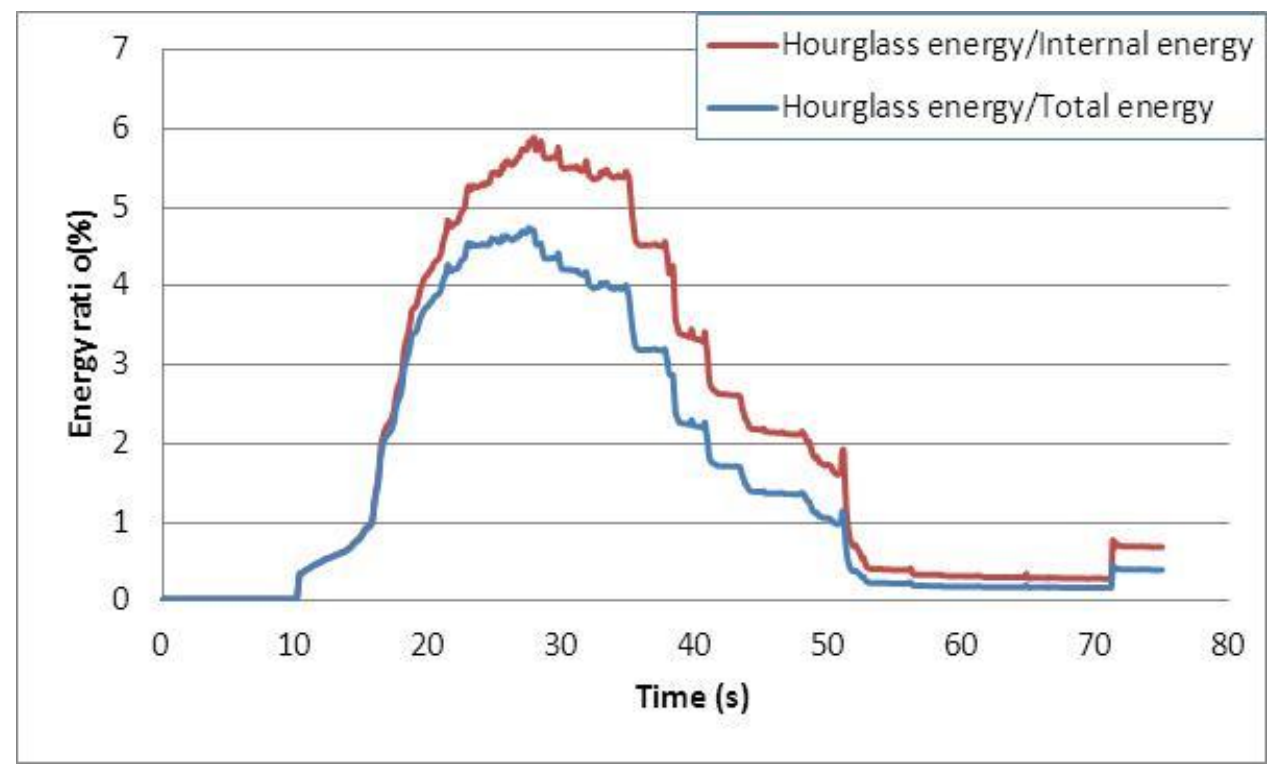

Figure 6-18 the ratio of hourglass and internal energy in the stenting model

\subsubsection{Loading and results}

Although applying axial stretch reduces the radius of the artery, it does not cause the artery to touch the stent before balloon expansion.

During balloon expansion, the stent comes in contact with the plaque surface (as well as the artery wall). As expansion increases the high stress area moves toward the middle part of the plaque, where the maximum pressure of the balloon is transferred to the plaque surface through the stent coils (Figure 6-19). The stress on the plaque surface is concentrated in two areas: where the middle coil of stent touches the plaque surface, and where the balloon touches the plaque surface, which is opposite to the site in contact with the stent's inner coil. This confirms that, unlike other stent types, the corners of the stent used in this study do not cause stress concentration on the arterial wall. At full expansion, the highest stress area remains in the middle of the plaque deposit (Figure 6-19). 
Time $=\quad 60$

Contours of Effective Stress (v-m)

max ipt. value

$\min =0.000947192$, at elem 12104327

max $=0.894627$, at eleme 12164239

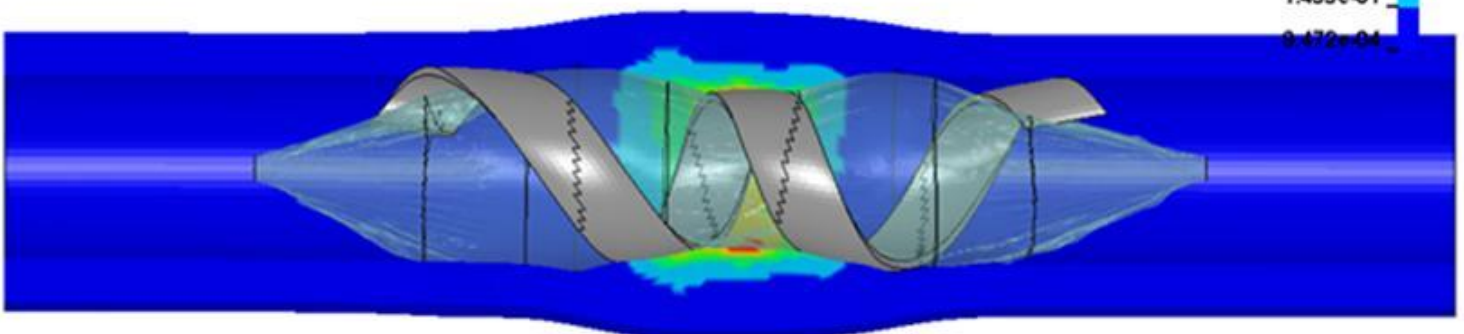

Stenting Cellular Plaque

Time $=131.6$

Contours of Effective Stress $(v-m)$

max ipt. value

$\min =0.00181023$, at elems 12086856

max $=2.47803$, at eleme 12160505

Fringe Levels

$2.478 \mathrm{e}+00$

$2.065 \mathrm{e}+00$

$1.653 \mathrm{e}+00$

$1.240 \mathrm{e}+00$

$8.272 \mathrm{e}-01$

4.145e-01

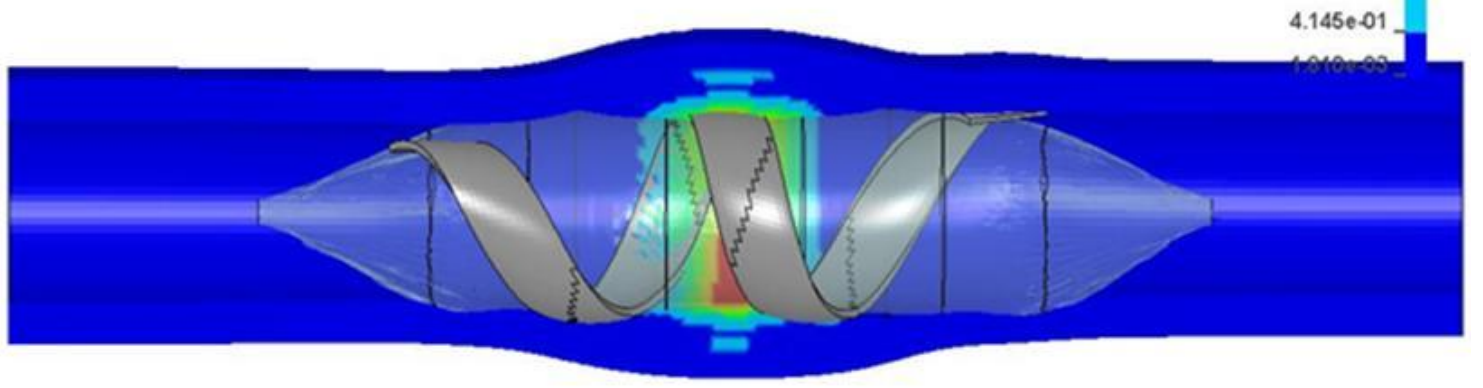

$r i_{x}^{2}$

Figure 6-19 von Mises stress distribution (MPa) of the artery and plaque before and after full stent expansion

The effective strain distribution also shows that the high strain area is concentrated in the middle part of the artery (Figure 6-20). The material model does not include defined failure points; therefore, the plaque and artery can potentially be strained beyond the failure points detected during experimentation. However, a sharp strain hardening area develops at the end of 
the hyperelastic model; straining the artery beyond this point is much more difficult than straining a non-strained part of the artery and plaque. Therefore, the area that passes the failure point is very limited and does not affect the validity of the analysis.

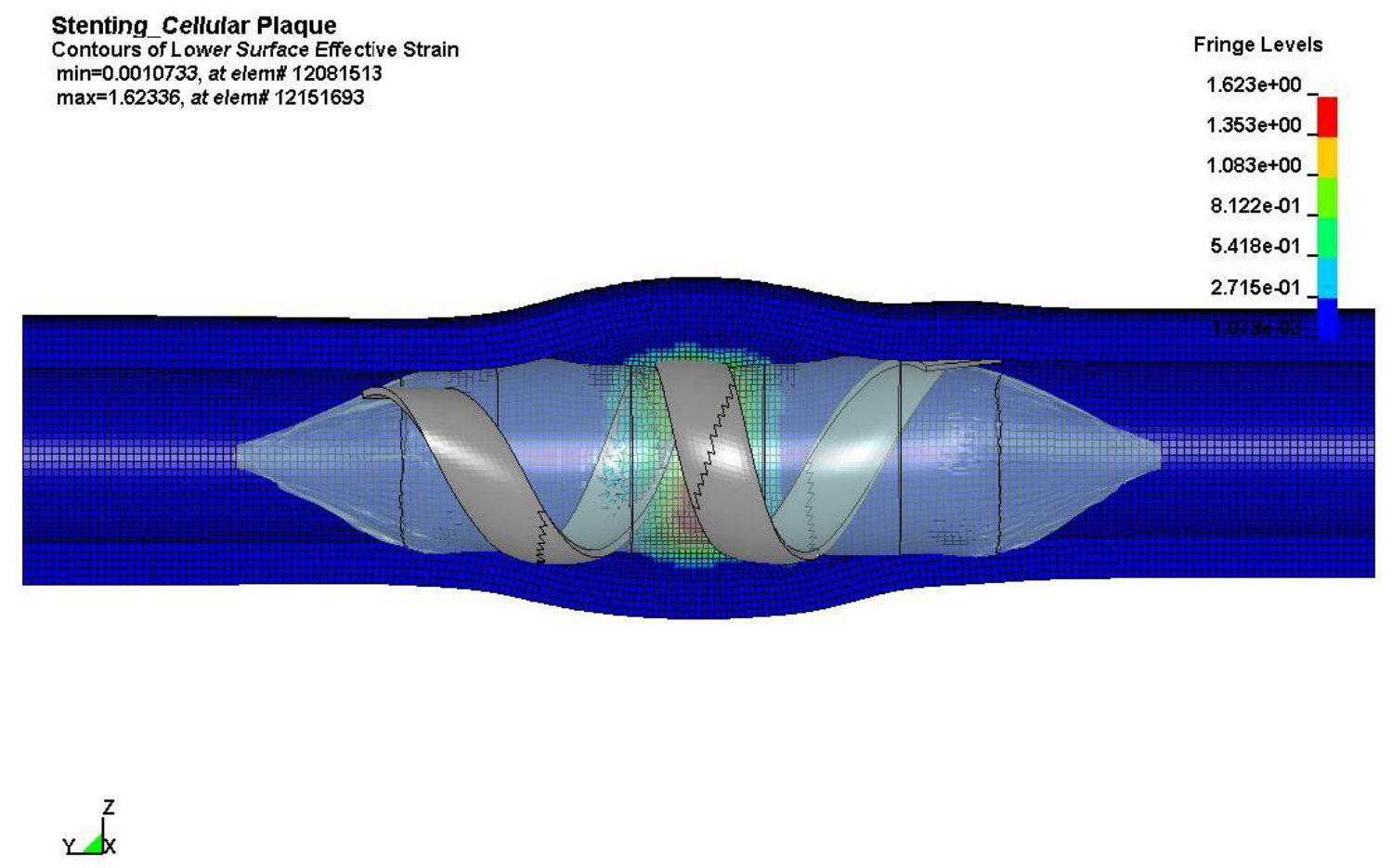

Figure 6-20 Effective strain distribution at balloon full expansion in artery and plaque

The stent touches the plaque surface after 20 seconds of loading. Figure 6-24 shows the von Mises stress distribution of the stent when fully expanded. The high stress area is at the inner surface where the stent touches the balloon. Although the artery puts some pressure on the end coils of the stent, signs of dog boning are evident during expansion. However, the observed dog boning was not severe enough to cause a stress concentration at the artery wall next to the end coils. The ends of the stent experience small stress levels that can produce significant recoiling after balloon removal, which can be considered a problem for this type of stent. This stress distribution profile was observed in the free expansion model (in the absence of a surrounding artery) as well (Chapter 5.3). This means the presence of an artery and plaque did not improve the high recoiling observed during free expansion. Therefore, the recoiling step was not analyzed in this chapter. 


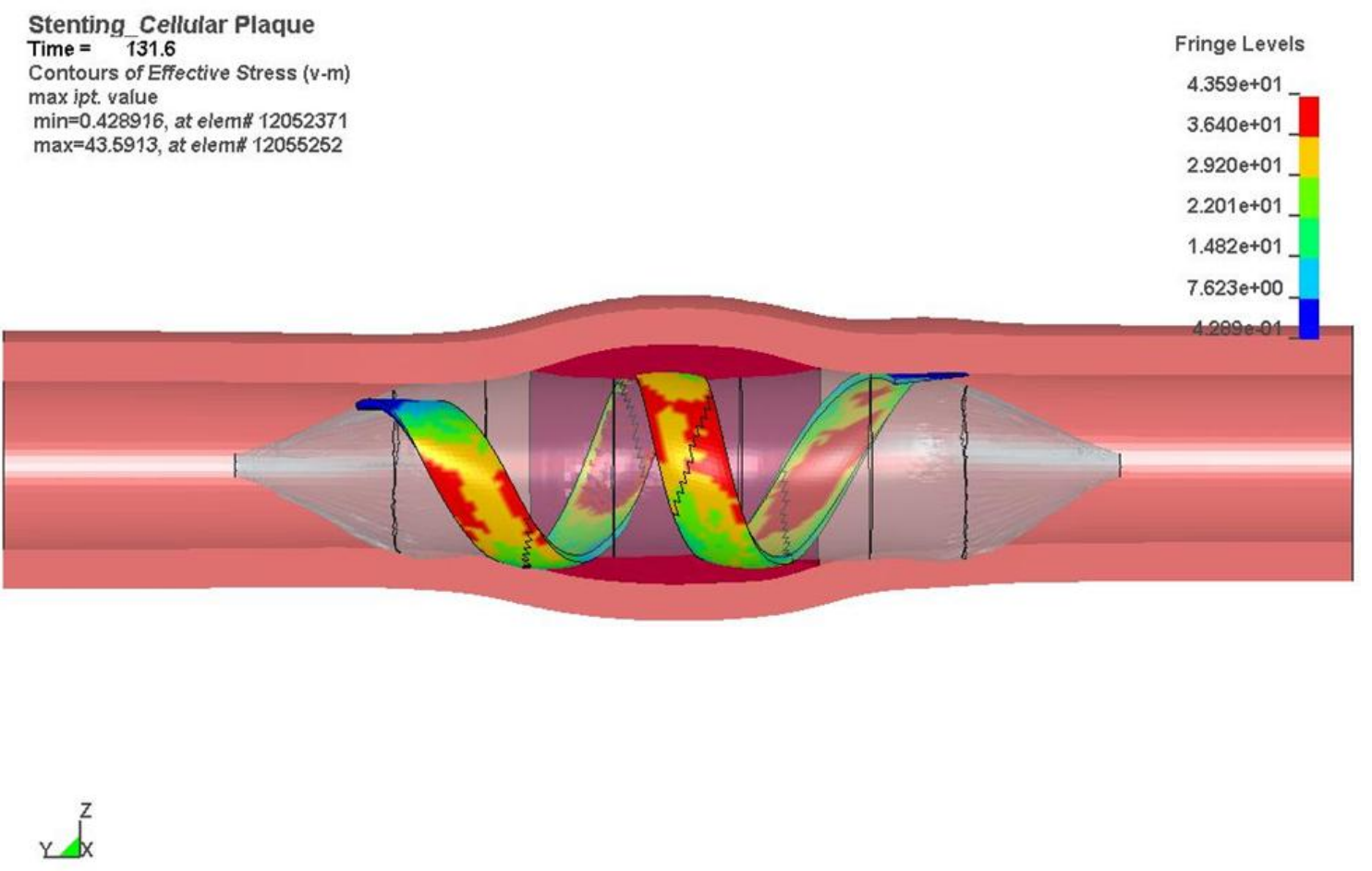

Figure 6-21 von Mises stress (MPa) in the stent at full expansion

As was observed in the free expansion model, the highest stress areas experienced by a stent deployed within a plaque-filled artery are located along its inner surface. It can be concluded that the unfolding behaviour of the balloon is not a factor in the stress distribution that develops in the arterial wall as the stent expands; therefore, the stress distribution cannot effectively be changed by increasing the number of folds in the balloon, to 4 or 6 folds for example.

Uncoiling behaviour is one of the dominant mechanisms of helical stent expansion. Since the stent is asymmetrical, its uncoiling behaviour is also asymmetrical, which influences the symmetry of the stress distribution along the artery.

Some foreshortening was observed during expansion, as shown in Figure 6-22. Free expansion of the helical stent (stent balloon expansion in the absence of an artery) showed very high foreshortening values (50-60\% foreshortening during full stent expansion, Chapter 5), and was considered a shortcoming of the stent design. Helical stent expansion inside the artery 
decreased foreshortening values considerably, to $15 \%$ (Figure 6-22), in comparison to the stent foreshortening in free expansion.

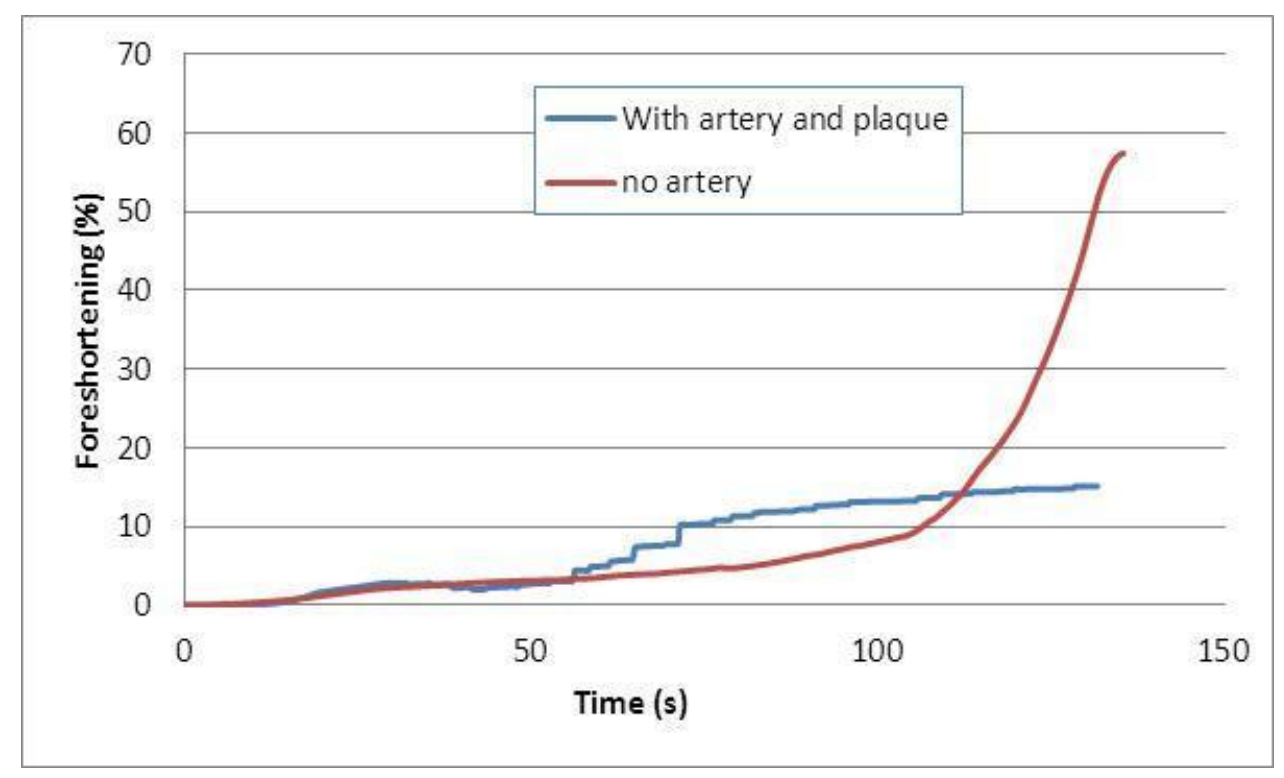

Figure 6-22 Foreshortening of the stent

\subsection{Potential injury areas in the artery and plaque post-expansion}

The von Mises stress distribution was used [Pant, 2012; Early, 2009; Karimi, 2013] to evaluate the areas in the angioplasty model (on both the artery and plaque) that exceed the failure point of the tested tissues. The threshold is defined as the last point defined in the experimental stress-stretch curve, corresponding to failure of the tissue in the experiment. When the von Mises stress in the artery or plaque reaches these points (300 and $650 \mathrm{KPa}$, respectively), the tissue is considered to be damaged or injured. Figures 6-23 and 6-24 show the von Mises stress distribution in the plaque and artery, respectively. The areas in which the von Mises stress exceeded the failure stress point of the material are marked in red. Since the stent configuration is asymmetrical, and therefore transfers only part of the balloon pressure to the artery and plaque, the potential injury areas on the artery and plaque are also asymmetrical. 


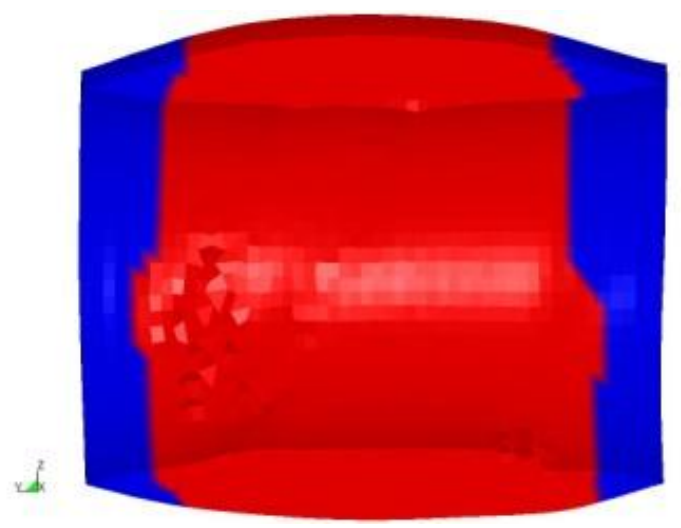

Figure 6-23 Potential injury areas on the plaque buildup following full expansion (shown in red)

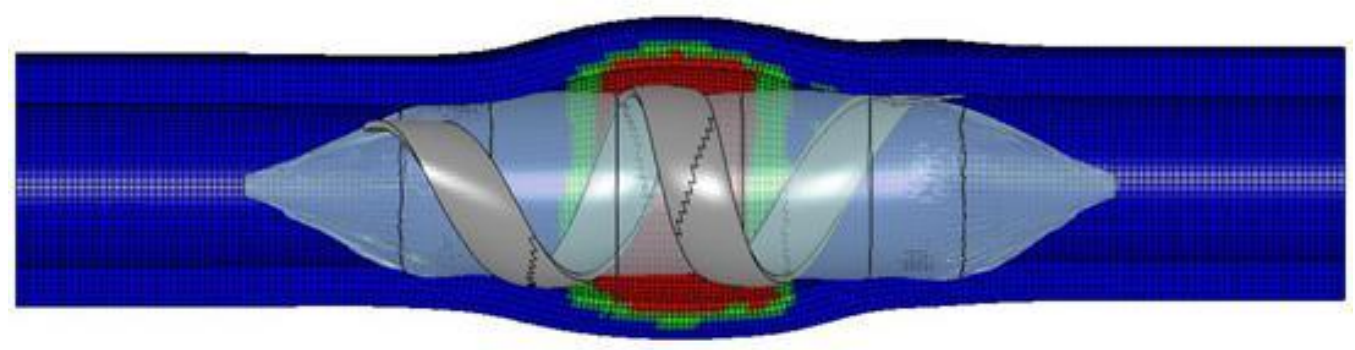

Figure 6-24 Artery volume at maximum balloon expansion in different stretch ranges (blue part: low stretch, green part: high stretch and red part: beyond the failure point measured in the experiment)

As expected, the artery may be damaged during expansion and stent support is required to prevent the injured wall from collapsing. Comparing the stress distribution and potential injury 
areas of angioplasty and the stenting model, it is evident that under the same expansion range the extent of injuries is greater in the stenting model. High recoiling was expected in this model as well, since the stress distribution was not improved with the presence of artery and plaque.

\subsection{Limitations of the study}

The elements of the artery and plaque that exceeded the failure stress threshold should be eliminated from the model as they cannot provide any mechanical contributions beyond that point. Material failure was not included in the model since there is limited data available and the intent of this study was to identify areas of high stress and strain that may potentially relate to injury of the artery.

The viscoelastic effects of the artery, plaque and stent material were not considered. However, relatively low deformation rates reduce these viscous effects. The shapes and sizes of both the artery and plaque structures are idealized and are not directly representative of real life samples. The artery and plaque materials were assumed to be homogenous, which may not be the case in real human samples.

Stent recoiling typically occurs after balloon deflation, but was not considered in the model. 


\section{Chapter 7}

\section{Summary and Conclusions}

This chapter summarizes the results of the material characterization experiments, finite element model development and analysis conclusions described in the body of this thesis. It also offers suggestions for future research directions to expand the work.

\subsection{Summary and Conclusions}

This study is part of a broader research initiative led by Nanyang technological university (NTU) in Singapore, which designed and fabricated a bilayer PLLA and PLGA biodegradable stent to alleviate the risk of restenosis following angioplasty and other cardiac interventions. The present work focused on evaluating this helical stent design, with particular emphasis on stent expansion and deployment characteristics. As a candidate for the next generation of biodegradable drug eluting stenting devices, the helical design has strong potential to resolve several shortcomings common to currently available stent technologies. However, it was reported (based on limited clinical observation) that this stent demonstrates high foreshortening (i.e., it shortens during expansion) and dog boning (i.e., expansion starts from the end coils) behaviour.

Based on the FE modeling presented in this thesis, during expansion by angioplasty balloon, the dog boning effect causes the stent coils to slide and overlap, which is not viable for the physical stent expansion, and leads to early termination of numerical simulations in nearly all cases. Polymeric helical stents differ considerably from currently used wire mesh designs, both in material composition and the expansion mechanisms occurring during deployment; therefore, present knowledge of stent design and implementation characteristics, which come primarily from wire mesh stent studies, cannot be applied directly to new helical devices.

This research was conducted to shed light on the expansion mechanism of balloon-expandable helical stents, the difference(s) between expansion of helical stents and more commonly used (and well-studied) wire mesh devices, and the effects these differences have on design solutions developed to resolve overlapping and dog boning issues experienced by helical stents. Due to the inherent complications and specifications of in vitro studies involving human tissue, computational methods (i.e., finite element modeling platforms including both implicit and explicit codes) were chosen to address these research objectives. The material properties used in 
the finite element codes were derived from in vitro experiments (presented in Chapter 3). The results of the experiments can be summarized as follows:

- In vitro conditions (i.e., contact body fluid and temperature) provide enough ductility in both PLLA and PLGA layers for proper stent expansion to the required diameter.

- PLGA samples lose their mechanical strength in in vitro conditions; therefore, this layer can be excluded from the model without affecting the results.

- PLLA samples have nonlinear viscoelastic properties; however, as expansion is very slow and almost quasi-static, elastic-plastic modeling of the stent does not affect the deployment results.

Finite element modeling and analysis of the results were the main part of this research. To find the best suited computational expansion and numerical analysis method, three common FEM expansions (expansion with inner pressure, uniform expansion of a high stiffness cylinder and folded balloon inflation) were examined for the helical stents. The numerical method (i.e., implicit and explicit) was chosen based on the complexity of the expansion. In Chapter 4, the development of each expansion method in FEM, results and the applicability of each method were discussed.

- The implicit method (using ABAQUS/Standard) is well suited for the first two expansion methods (i.e., inner pressure expansion and uniform expansion).

- The helical stent cannot be expanded properly with inner pressure. This method does not provide realistic axial support for the helical stent, which, in practice, is provided at the interface of the expanding balloon and the helical stent.

- The uniform expansion model was successful in expanding the stent. Although the forced boundary conditions eliminated the transient end-first expansion pattern (i.e., dog boning), the final expanded geometry was similar to the unique (and successful) expanded helical stent geometry reported by NTU. The experimental results (in both the expansion and recoiling stage) were used to validate this model.

The validated uniform expansion model was used for a series of parametric studies undertaken to optimize the helical stent geometry. The goal of the study was to minimize the evaluation parameters (e.g., foreshortening, dog boning and recoiling). As the expansion mechanism used by helical stents is different from common wire mesh stents, two new evaluation parameters 
(i.e., uncoiling and pitch change) were introduced in this study to highlight some of main characteristics of helical stents that cannot be captured with common stenting parameters. Due to relatively high foreshortening (more than 50\%), helical stents should have more than 3 coils in order to provide adequate artery support after expansion.

- Based on the results of the parametric study conducted using the uniform expansion models, foreshortening in the helical stents can be reduced by increasing the width or thickness of the stent while the pitch angle is constant.

- Recoiling of the helical stent is dependent on the level of plastic strain induced during the expansion process. This fact is similar to the other type of balloon expandable stents.

- The stent-balloon expansion modeling has number of complicated contact surfaces. The presence of this nonlinearity necessitates the use of explicit code (i.e., LS Dyna). This model is capable of revealing the end-first dog boning expansion pattern and consequently the overlapping problem in the helical stent. The expansion profile is qualitatively similar to the experimental results.

To solve the dog boning problem, a series of parametric studies were conducted on the stentfolded balloon expansion model. The process of analysis and the conclusions driven from them were presented in Chapter 5, and are briefly as follows:

- Changes in stent geometry (stent width, thickness, number of coils and length) did not reduce the dog boning problem.

- Localized changes in stent geometry produced by reducing the width and thickness at both ends of the stent were not effective in reducing dog boning behaviour.

- Changes at the stent-balloon interface (i.e., by changing the COF at the vicinity of the ending coils) reduced dog boning and improved the expansion. Also localized strengthening the balloon at two ends slightly decreased the dog bone effect.

- Changing the design of the helical stent by using repetitive three-coil stent along the stent which are connected with a connector part can solve the expansion problem. This stent however, is weak in radial direction after expansion.

- Applying a progressive pressurizing pattern to the balloon, where the balloon starts to expands from the middle part of balloon (and consequently the stent) reduce dog boning and solved the expansion problem of the helical stents. This pattern of pressurizing can be used for the other stents as well, to reduce dog boning. 
Free expansion analysis is always considered the preliminary step for stent deployment studies. In Chapter 6, the proposed progressive pressurization pattern was used to expand and deploy a 5-coil stent inside an idealized artery with crescent-shape plaque buildup.

To provide a frame of reference to study the stress distribution on artery and plaque after expansion, an angioplasty procedure (with and without the presence of plaque) was also modeled and analyzed in Chapter 6. The axial stretch in the artery as reported in the literature was also considered. These studies revealed the following:

- By considering the axial stretch in the artery, the final von Mises stress distributions are higher in both the artery and plaque, compared to the model without axial stretch.

- Expanding the helical stent inside the artery reduces the foreshortening and dog boning of stent.

- A comparison of the stenting and angioplasty models indicates that the presence and expansion of a helical stent inside an artery imposes much greater stress on the arterial wall than does the angioplasty procedure alone.

- The presence of stent causes non uniform expansion in the artery which leads to asymmetrical stress distribution in the artery.

\subsection{Contributions and future work}

In general, the present study yielded a number of contributions to our understanding of polymeric helical stent design and expansion mechanisms through both experimental and, in particular, computational studies. These contributions are briefly as follows:

- In vitro conditions improve the deformation and ductility properties of PLLA samples.

- Helical stent expansion occurs via a different mechanism than is used by common wire mesh stents. Therefore, available data on wire mesh stents are not applicable to helical stent studies.

- Simple expansion method such as applying inner pressure is not suitable to analysis a helical stent

- The uniform expansion method is able to present a first order result on the expansion pattern of a helical stent. This method, however, is not suitable for complete analysis of helical stent expansion. 
- Stent-folded balloon expansion modeling is well-suited to address the complexities of helical stent expansion analysis. Parametric studies using this model suggested that a progressive pressurization pattern can improve and solve the main expansion problem experienced by helical stents (i.e., dog boning).

- Deployment of the stent inside the artery improves the foreshortening of the helical stent.

- The artery moves and twists along with the stent expansion, which causes asymmetrical artery geometry.

This study aimed to evaluate and optimize helical stent deployment using finite element modeling methods. The following are suggested extensions of the current work that may help advance our understanding of helical stent expansion:

- Adding the viscous properties to the stent and studying the recoiling of stent for successful deployment. Since the glass transition temperature of the PLLA layer was less than $20^{\circ} \mathrm{C}$ above body temperature, creep in stent coils was anticipated after deployment in artery. Therefore, to study the long term behaviour of this type of stent, a study on the creep response is necessary.

- Using patient-specific artery and plaque geometries and material properties.

- Expanding the helical stent inside a curved artery. 


\section{Appendix A \\ Helical Stent Flexibility Investigation}

We evaluated the flexibility of the helical stent design using the same model as described in4.4. For the simulation, both ends of the stent were fixed in the x-direction (Figure A-1) and a concentrated force was applied at the middle of the stent in the x-direction. The force was increased until both ends of the stent touched each other and formed a full circle. Then, the load was removed to allow the stent to resume to its original shape without any plastic deformation. The maximum von Mises stress was $10 \mathrm{MPa}$, which was well below the yield strength of the material and demonstrates that helical stents can undergo significant bending during the implantation process without adverse effects on the stent.
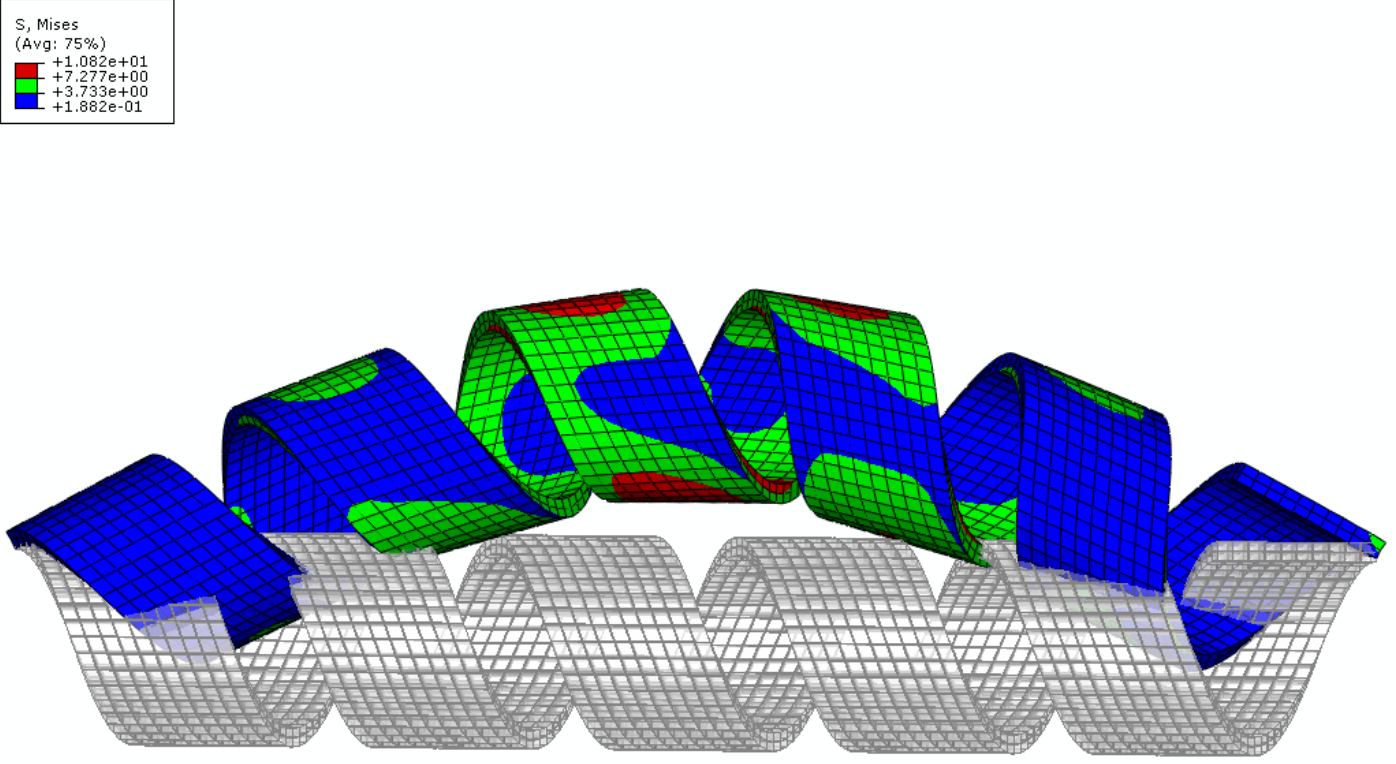

ODB: unloding.odb Abaqus/Standard Version 6.7-1 wed Oct 01 19:20:29 Eastern Daylight Time 2008

Step: Step-1

Increment 19: Step Time $=0.1290$

Primary Var: S, Mises
Deformed Var: $U$ Deformation Scale Factor: $+1.000 e+00$

Figure A- 1 The FEM model of helical stent flexibility. 


\section{Appendix B \\ Implicit versus explicit finite element modeling approach for analyzing stent uniform expansion}

To discern the effect of the analysis method on the straining of the stent during expansion, a stenting expansion problem was solved using both implicit (ABAQUS/Standard, Simulia) and explicit (LS-Dyna, LSTC) finite element solvers and assuming the same geometry, mesh, material properties, and interaction formulations as was applied in both models. Initially, no mass or scaling was applied to the LS-Dyna model in this case; however, scaling was included later and did not significantly affect the results.

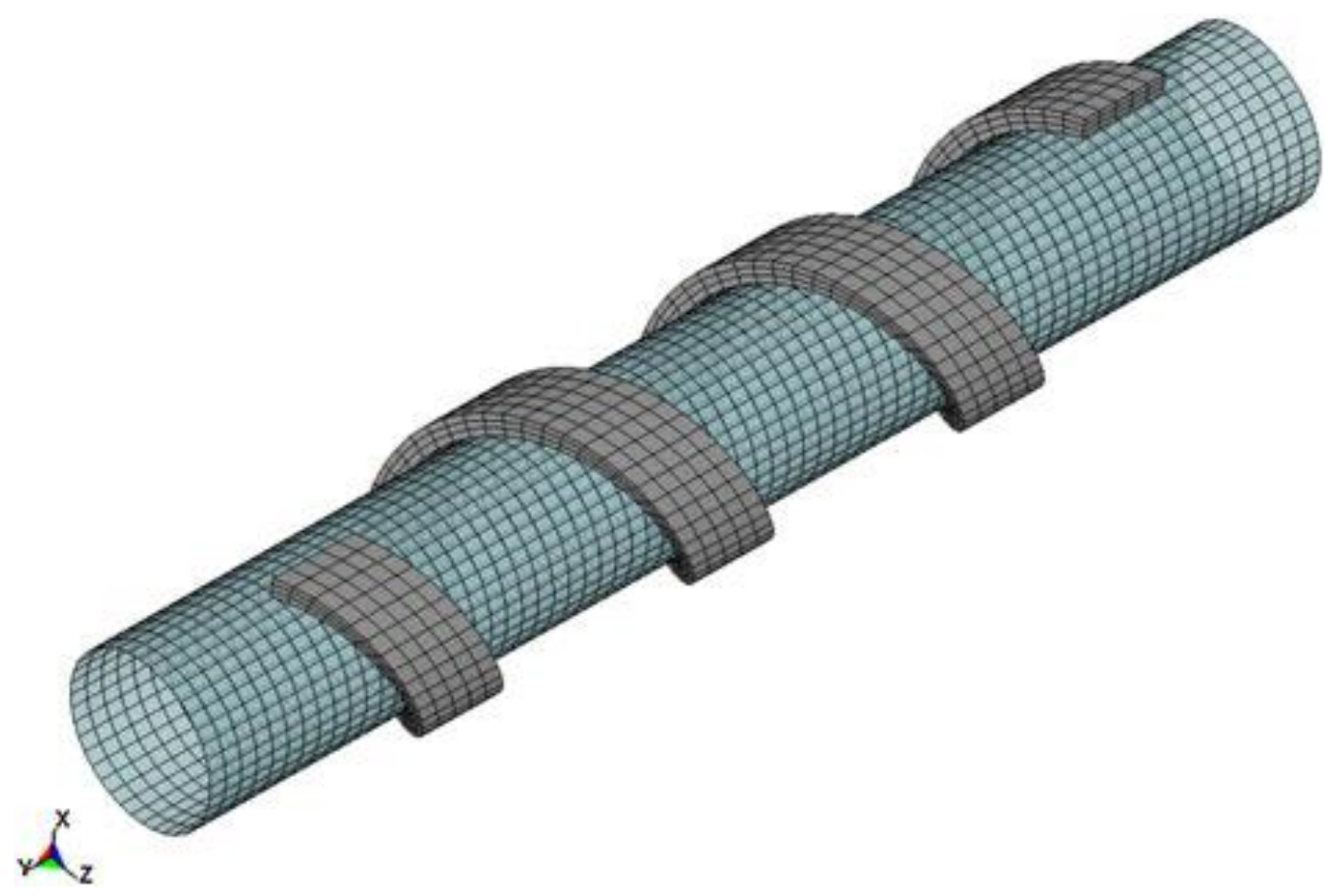

Figure B- 1 Mesh and geometry of the stent and expansion cylinder.

A three-coil stent with an inner diameter of $3.0 \mathrm{~mm}$, thickness of $0.12 \mathrm{~mm}$, width of $0.5 \mathrm{~mm}$, and pitch value of $2.0 \mathrm{~mm}$ was expanded by $0.4 \mathrm{~mm}$ in the radial direction using a cylinder (Figure B-1). The cylinder was expanded in the radial direction and after coming in contact with 
the stent expanded the stent coils, while allowing for axial motion and uncoiling. Performance parameters predicted by the two numerical methods (i.e., implicit and explicit) were compared using common metrics. Generally, the mechanical performance of helical stents is measured based on key evaluation parameters such as foreshortening and uncoiling [Paryab, 2012], with smaller values generally accepted as indicative of better performance. Foreshortening is a measure of the change in the length of the stent during expansion, and is defined as follows [Kiousis, 2009]:

Foreshortening $=\frac{L_{1}-L_{2}}{L_{1}} \times 100$

Equation B- 1

where $\mathrm{L} 1$ is the original length and L2 is the final length.

Uncoiling behaviour is determined by measuring the number of coils before and after stent expansion, and is defined as follows [Paryab, 2012]:

Uncoiling $=\frac{\# \text { Coils }_{0}-\# \text { Coils }_{f}}{\# \text { Coils }_{0}} \times 100$

Equation B- 2

where \# coils and \# coilsf are the initial and final number of coils, respectively.

Expansion is defined as follows:

Expansion $\%=\frac{R_{\text {Final }}-R_{\text {initial }}}{R_{\text {initial }}} \times 100$

Equation B- 3

where $\mathrm{R}$ is the inner radius of the stent. 


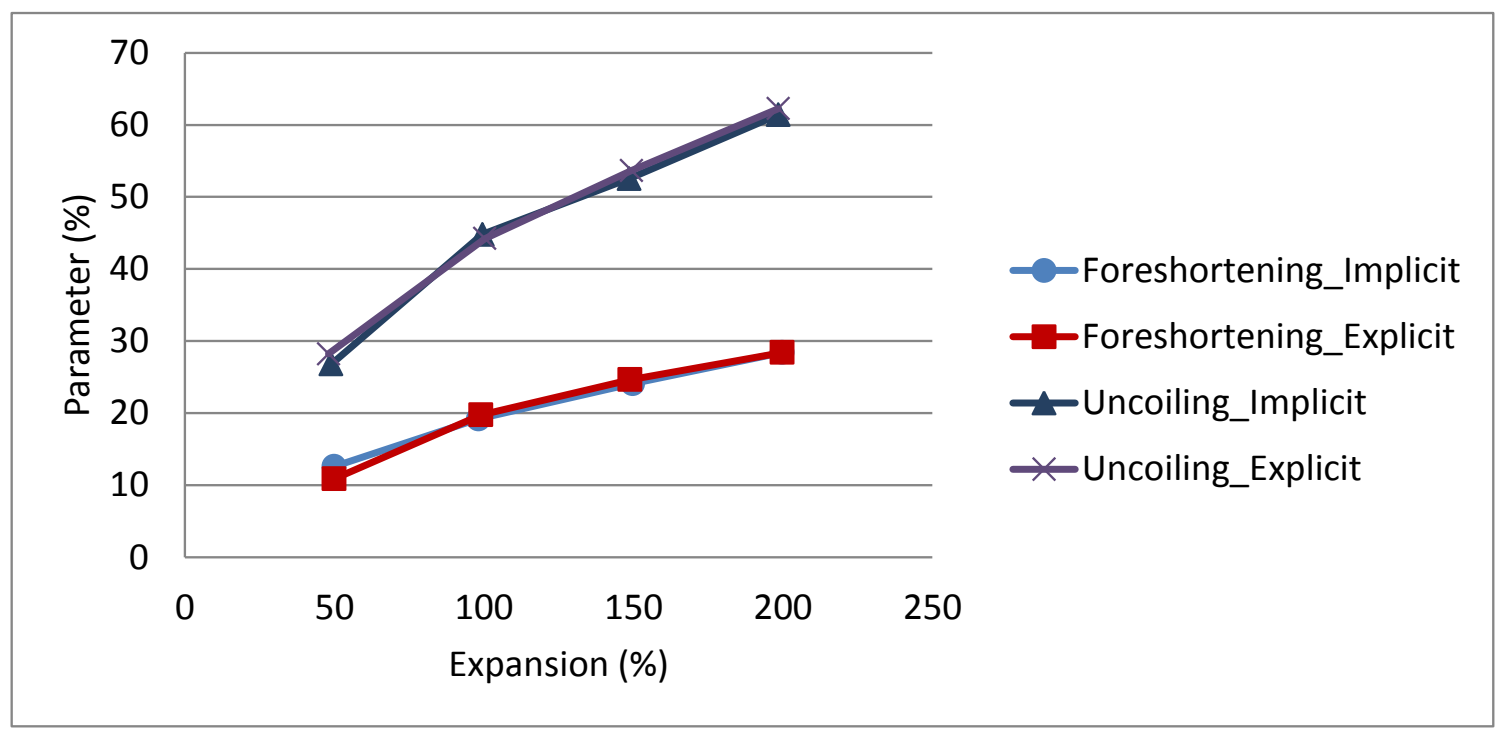

Figure B- 2 Comparison of stent expansion response using implicit and explicit finite element solvers.

It was found that both the implicit and explicit codes predicted similar results (Figure B-2) for the uniform expansion model. The implicit code provided reduced computation time for this problem; however, only the explicit code could be used for the more complicated balloon expansion problem, which included contacts and deformations. 


\section{Bibliography}

[ABAQUS Manual] ABAQUS Manual

[ACCF/AHA/SCAI Guideline, 2011] writing committee members: Levine, G.N., Bates, E.R., Blankenship, J.C., Bailey, S.R., Bittl, J.A., Cercek, B., Chambers, C.E., Ellis, S.G., Guyton, R.A., Hollenberg, S.M., Khot, U.N., Lange, R.A., Mauri, L., Mehran, R., Moussa, I.D., Mukherjee, D., Nallamothu, B.K., and Ting, H.H., 2011, “ACCF/AHA/SCAI guideline for percutaneous coronary intervention a report of the american college of cardiology foundation/american heart association task force on practice guidelines and the society for cardiovascular angiography and interventions," Circulation, 124, e574-e651.)

[Agrawal, 1992] Agrawal, C.M., Haas, K, F., Leopold, D.A., and Clark, H.G., 1992, "Evaluation of ploy (l-lactic acid) as a material for intravascular polymeric stents," Biomaterials, 13.3, pp. 176-182.

[Athanasiou, 1996] Athanasiou, K.A., Niederauer, G.G., and Agrawal, C.M., 1996, "Sterilization, toxicity, biocompatibility and clinical applications of polylactic acid/ polyglycolic acid copolymers," Biomaterials, 17, pp.93-102.

[Auricchio 2001] AURICCHIO, F., Di Loreto, M., and Sacco, E., 2001, "Finite-element analysis of a stenotic artery revascularization through a stent insertion", Computer Methods in Biomechanics and Biomedical Engineering, 4(3), pp. 249-263.

[Ballyk, 2006] Ballyk, P.D., 2006, "Intramural stress increases exponentially with stent diameter: a stress threshold for neointimal hyperplasia,” Journal of Vascular and Interventional Radiology, 17(7), P. 1139-1145.

[BCC research] BCC research on The Market for Minimally Invasive Medical Devices (http://www.bccresearch.com/report/minimally-invasive-devices-market-hlc051f.html last accessed Feb 13, 2012)

[Bedoya, 2006] Bedoya, J., Meyer ,C.A., Timmins, L.H., Moreno, M.R., and Moore, J.E., 2006, "Effect of stent design parameters on normal artery wall mechanics," Journal of biomechanical engineering, 128, pp. 757-765.

[Belytschko, 1984] Belytschko, T., Lin, J. I., and Chen-Shyh, T., 1984, "Explicit algorithms for the nonlinear dynamics of shells," Computer methods in applied mechanics and engineering, 42(2), 225-251. 
[Berry, 2002] Berry, J.L., Manoach, E., Mekkaoui, C., Rolland, P.H., Moore Jr., J.E., Rachev, A., 2002, "Hemodynamics and wall mechanics of a compliance matching stent: in vitro and in vivo analysis,” Journal of Vascular and Interventional Radiology, 13(1), pp.97-105.

[bostonscientific.com] http://www.bostonscientific.com/lifebeat-online/heart-smart/how-yourheart-works.html

[Boyle, 2010] Boyle, C. J., Lennon, A. B., Early, M., Kelly, D. J., Lally, C., and Prendergast, P. J., 2010, “Computational simulation methodologies for mechanobiological modelling: a cell-centred approach to neointima development in stents," Philosophical Transactions of the Royal Society A: Mathematical, Physical and Engineering Sciences, 368(1921), pp. 29192935.

[Bravo] Bravo NM coronary dilatation catheter, Neich Medial [Bressloff, 2013] Bressloff, Neil W., 2013, "Multi-objective design of a biodegradable coronary artery stent," Stud Mechanobiol Tissue Eng Biomater, pp. 1-28.

[Briguori, 2002] Briguori, C., Sarais, C., Pagnotta, P., Liistro, F., Montorfano, M., Chieffo, A., Sgura, F., Corvaja, N., Albiero, R., Stankovic, G., Toutoutzas, C., Bonizzoni, E., Di Mario, C., and Colombo, A., 2002, "In-stent restenosis in small coronary arteries: impact of strut thickness," Journal of the American College of Cardiology, 40, pp. 403- 409.

[Brinson, 2008] Brinson, H. F., and Brinson, L. C., 2007, "Polymer engineering science and viscoelasticity: an introduction," Springer.

[Bund, 1996] Bund, S. J., Oldham, A. A., and Heagerty, A. M., 1996, “Mechanical properties of porcine small coronary arteries in one-kidney, one-clip hypertension," Journal of vascular research, 33(2), pp. 175-180.

[Bünger, 2007] Bünger, C.M., Grabow, N., Sternberg, K., Kröger, C., Ketner, L., Schmitz, K.P., Kreutzer, H.J., Ince, H., Nienaber, C.A., Klar, E., and Schareck, W., 2007, "Sirolimuseluting biodegradable poly-l-lactide stent for peripheral vascular application: a preliminary study in porcine carotid arteries", Journal of Surgical Research 139, 77-82

[Burt, 2006] Burt, H., and Hunter, W., 2006, "Drug-eluting stents: a multidisciplinary success story," Advanced Drug Delivery Reviews, 58, pp.350-357.

[Canham, 1989] Canham, P.B., Finlay, H.M., Dixon, J.G., Boughner D.R., and Chen, A., 1989, "Measurements from light and polarised light microscopy of human coronary arteries fixed at distending pressure." Cardiovasc Res, 23, pp.973-982. 
[Capelli, 2009] Capelli, C., Gervaso, F., Petrini, L., Dubini, G., and Migliavacca, F., 2009, "Assessment of tissue prolapse after balloon-expandable stenting: Influence of stent cell geometry," Medical Engineering and Physics (Finite Element Modelling of Medical Devices), 31(4), pp. 441-447.

[Carew, 1968] Carew, T.E., Vaishnav, R.N., and Patel, D.J., 1968, “Compressibility of the arterial wall," Circulation Research, 22, pp. 61-68.

[Christensen, 1982] Christensen, R.M., 1982, “Theory of Viscoelasticity," $2^{\text {nd }}$ edition, Academic Press, New York,

[Chu, 1985] Chu, C.C., 1985, "Strain-accelerated hydrolytic degradation of synthetic absorbable sutures, in: C.W. Hall, (Ed), Surgical research, recent developments," Proceedings of the First Annual Scientific Session of the Academy of Surgical Research, San Antonio, pp. $111-115$

[Chua, 2004] Chua, S.N.D., MacDonald B.J., and Hashmi M.S.J., 2004, "Finite element simulation of slotted tube (stent) with the presence of plaque and artery by balloon expansion,” Journal of Materials Processing Technology, 155-156, pp.1772-1779.

[Chung, 1998] Chung, W.J., Cho, J.W., and Belyschko, T., 1998, “on the dynamic effects of explicit FEM in sheet metal forming analysis," engineering computations, 15, pp. 750-776. [Claes, 2010] Claes, E., Atienza, J.M., Guinea, G.V., Rojo, F.J., Bernal, J.M., Revuelta, J.M., and Elices, M., 2010, "Mechanical properties of human coronary arteries," In Engineering in Medicine and Biology Society (EMBC), 2010 Annual International Conference of the IEEE, pp. 3792-3795.

[Communication with NTU] A personal communication with NTU with biodegradable helical stent designer in Material department of Nanyang Technological university (NTU) [Christensen, 1980] Christensen, R. M.,1980, “A nonlinear theory of viscoelasticity for application to elastomers," Journal of Applied Mechanics, 47(4), pp.762-768.

[De Beulea, 2008] De Beule, M., Mortier, P., Carlier, S. G., Verhegghe, B., Van Impe, R., and Verdonck, P., 2008, "Realistic finite element-based stent design: the impact of balloon folding," Journal of Biomechanics, 41(2), pp. 383-389.

[Dobrin, 1969] Dobrin, P.B., Rovick, A.A., 1969, "Influence of vascular smooth muscle on contractile mechanics and elasticity of arteries," American Journal of Physiology, 217, pp. 1644-1651. 
[Dumoulin 2000] Dumoulin, C., and Cochelin, B., 2000, "Mechanical behaviour modelling of balloon-expandable stents," Journal of Biomechanics, 33(11), pp.1461-1470.

[Early, 2008] Early, M., Lally, C., Prendergast, P.J., and Kelly, D.J., 2008, “Stresses in peripheral arteries following stent placement: a finite element analysis," Computer Methods in Biomechanics and Biomedical Engineering, 12, pp. 25-33.

[Elakkiya 2013] Elakkiya, T., Sheeja, R., Ramadhar, K., \& Natarajan, T. S., 2013, "Biocompatibility studies of electrospun nanofibrous membrane of PLLA-PVA blend," Journal of Applied Polymer Science, 128(5), pp. 2840-2846.

[Etavea, 2001] Etavea, F., Finet, G., Boivina, M., Boyera, J., Rioufolb, G., Thollet, G., 2001, "Mechanical properties of coronary stents determined by using finite element analysis," Journal of Biomechanics, 34, pp. 1065-1075.

[Falk, 1995] Falk, E., Shah, P.K., and Fuster, V., 1995, "Coronary plaque disruption," Circulation, 92, pp. 657-671.

[Fernández-Ortiz, 1994] Fernández-Ortiz, A., Badimon, J., Falk, E., Fuster ,V., Meyer, B., Mailhac, A., Weng, D., Shah, P.K, Badimon, L., 1994, "Characterization of the relative thrombogenicity of atherosclerotic plaque components: implications for consequences of plaque rupture,” Journal of American Coll Cardioliology, 23, pp.1562-1569.

[Ferry, 1970] Ferry, J.D., 1970, Viscoelastic Properties of polymers, 2nd edition, J. Wiley, New York,

[Fisher, 2007] Fisher, J. P., Mikos, Joseph, A. G., and Bronzino D., 2007, “Tissue engineering," CRC press, pp. 8-6.

[Frank, 2004] Frank, A., Kumar Rath, S., Boey, F., and Venkatraman, S., 2004, "Study of the initial stages of drug release from a degradable matrix of poly (d,1-lactide-co-glycolide)," Biomaterials, 25(5), pp. 813-821.

[Friction Coefficients] Friction Coefficients, http://www.engineershandbook.com. Last accessed January 9, 2012

[Gastaldi, 2010] Gastaldi, D., Morlacchi, S., Nichetti, R., Capelli, C., Dubini, G., Petrini, L., Migliavacca, F., 2010, "Modelling of the provisional side-branch stenting approach for the treatment of atherosclerotic coronary bifurcations: effects of stent positioning," Biomech Model Mechanobiol, 9, pp. 551-561. 
[Gervaso, 2008] Gervaso, F., Capelli, C., Petrini, L., Lattanzio, S., Virgilio, L.D., Migliavacca F., 2008, "On the effects of different strategies in modelling balloon-expandable stenting by means of finite element method," Journal of Biomechanics, 41, pp. 1206-1212.

[Gijsen, 2008] Gijsen, F.J.H., Migliavacca, F., Schievano, S., Socci, L., Petrini, L., Thury, A., Wentzel, J.J., van der Steen, A.F.W., Serruys, P.W.S., and Dubini, G., 2008, "Simulation of stent deployment in a realistic human coronary artery," BioMedical Engineering On Line 2008, 7(23), pp.1-11.

[Gow, 1974] Gow, B.S., Schonfeld, D., and Patel, D.J., 1974, "The dynamic elastic properties of the canine left circumflex coronary artery," J Biomech, 7, pp. 389-395.

[Grabow, 2005] Grabow, N., Schlun, M., Sternberg, K., Hakansson, N., Kramer, S., Schmitz, K.P., 2005, "Mechanical properties of laser cut poly(l-lactide) micro-specimens: implications for stent design, manufacture, and sterilization," Journal of Biomechanical Engineering, 127, pp. 25-31.

[Grabow, 2007] Grabow, N., Bunger, C.M., Schultze, C., Schmohl, K., Martin, D.P., Williams, S.F., Sternberg, K., and Schmitz, K.P., 2007, “A biodegradable slotted tube stent based on poly(l-lactide) and poly(4-hydroxybutyrate) for rapid balloon-expansion," Annals of Biomedical Engineering, 35 (12), pp. 2031-2038.

[Grogan, 2011] Grogan, J.A., O’Brien, B.J., Leen, S.B., McHugh, P.E., 2011, “A corrosion model for bioabsorbable metallic stents," Acta Biomaterialia, 7(9), pp. 3523-3533.

[Gu 2010] Gu, L., Zhao, S., Muttyam, A. K., and Hammel, J. M., 2010, “The relation between the arterial stress and restenosis rate after coronary stenting," Journal of Medical Devices, 4(3), pp. 031005.

[Hall, 2006] Hall, G.J., and Kasper, E.P., 2006, “Comparison of element technologies for modelling stent expansion,” Journal of Biomechanical Engineering, 128, pp.751-756.

[Hausleiter, 2002] Hausleiter, J., Sebastian, M., Li A.N., Abbey, C,K., Honda, H., Makkar, R., Whiting, J.S., and Eigler, N., 2002, “A porcine coronary stent model of increased neointima formation in the left anterior descending coronary artery," Z Kardiol, 91, pp. 614-619.

[Hayashi, 1997] Hayashi, K., and Imai, Y., 1997, "Tensile property of atheromatous plaque and an analysis of stress in atherosclerotic wall," Journal of biomechanics, 30, PP. 573-579. [Hibbeler, 1999] Hibbeler, R.C., 1999, "Mechanics of Materials”, Fourth edition, Prentice Hall, New Jersey. 
[Hirai, 1989] Hirai, T., Sasayama, S., Kawasaki, T., \& Yagi, S., 1989, "Stiffness of systemic arteries in patients with myocardial infarction. A noninvasive method to predict severity of coronary atherosclerosis," Circulation, 80(1), pp. 78-86.

[Holzapfel, 2000] Holzapfel, G. A., Schulze-Bauer, C. A., and Stadler, M., 2000, "Mechanics of angioplasty: Wall, balloon and stent," ASME Applied Mechanics Division-PublicationsAMD, 242, pp. 141-156.

[Holzapfel, 2004] Holzapfel, G.A., Sommer, G., and Regitnig P., 2004, “Anisotropic mechanical properties of tissue components in human atherosclerotic plaques," ASME Journal of Biomechanical Engineering, 126, pp. 657-665.

[Holzapfel, 2005] Holzapfel, G. A., Sommer, G., Gasser, C. T., and Regitnig, P., 2005, "Determination of layer-specific mechanical properties," Circulation, 289, pp. H2048-2058. [Ju, 2008] Ju, F., Xia, Z., and Sasaki, K., 2008, “On the finite element modelling of balloonexpandable stents," journal of the mechanical behaviour of biomedical material, 1, 86-95. [Kandzari, 2002] Kandzari, D.E., Tcheng, J.E., and Zidar, J.P., 2002, “Coronary artery stents: evaluating new designs for contemporary percutaneous intervention," Catheterization and Cardiovascular Interventions, 56, pp. 562-576.

[Kang, 1995] Kang, T., Resar, J., and Humphrey, J.D., 1995, Heat-induced changes in the mechanical behaviour of passive coronary arteries," J Biomech Eng, 117, pp. 86-93.

[Kang, 1998] Kang, S. H., Park, H. K., Lee, C. W., Kim, J. J., Hong, M. K., Park, S. W., and Park, S. J., 1998, “Impaired flow-mediated vasodilation of epicardial coronary artery in vasospastic angina,” Journal of Korean medical science, 13, pp.591-596.

[Karimi, 2008] Karimi, R., Zhu, T., Bouma, B.E., Kaazempur Mofrad, M.R., 2008, "Estimation of nonlinear mechanical properties of vascular tissues via elastography," Cardiovasc Eng, 8, pp. 191-202.

[Karimi, 2013] Karimi, A., Navidbakhsh, M., Faghihi, S., Shojaei A., and Hassani, K., 2013, "A finite element investigation on plaque vulnerability in realistic healthy and atherosclerotic human coronary arteries," Proc IMechE Part H: J Engineering in Medicine, 227(2), pp. 148161.

[Kastrati, 1997] Kastrati, A., Schomig, A., Elezi, S., Schu“hlen, H., Dirschinger, J., Hadamitzky, M., Wehinger, A., Hausleiter, J., Walter, H., and Neumann, F.J., 1997, "Predictive factors of restenosis after coronary stent placement," J Am Coll Cardiol, 30, pp. $1428-1436$. 
[Kiousis, 2007] Kiousis, D.E., Gasser, T.C., and Holzapfel, G.A., 2007, “A numerical model to study the interaction of vascular stents with human atherosclerotic lesions", Annals of Biomedical Engineering, 35(11), pp. 1857-1869.

[Kiousis, 2009] Kiousis, D.E., Wulff, A.R., and Holzapfel, G.A., 2009, “Experimental studies and numerical analysis of the inflation and interaction of vascular balloon catheter-stent systems," Annals of Biomedical Engineering, 37, pp. 315-330.

[Kragel, 1989] Kragel A.H., Shanthasundari G., Reddy S.G., Wittes J.T., Roberts W.C., 1989, "Morphometric analysis of the composition of atherosclerotic plaques in the four major epicardial coronary arteries in acute myocardial infarction and in sudden coronary death," Circulation, 80, pp. 1747-1756.

[Kural, 2012] Kural, M.H., Cai, M., Tang, D., Gwyther, T., Zheng, J., and Billiar, K.L., 2012, "Planar biaxial characterization of diseased human coronary and carotid arteries for computational modeling,” J Biomech., 45(5), pp. 790-798.

[Lally, 2004] Lally, C., Reid, A., and Prendergast, P., 2004, "Elastic behaviour of porcine coronary artery tissue under uniaxial and equibiaxial tension,” Ann Biomed Eng., 32, pp. 1355-1364.

[Lally, 2005] Lally, C, Dolan, F., and Prendergast, P., 2005, “ Cardiovascular stent design and vessel stresses: a finite element analysis," Journal of Biomechanics, 38, pp. 1574-1581. [Lansky, 2000] Lansky, A. J., Roubin, G. S., O’Shaughnessy, C. D., Moore, P. B., Dean, L. S., Raizner, A. E., ... and Leon, M. B., 2000, "Randomized comparison of GR-II stent and Palmaz-Schatz stent for elective treatment of coronary stenosis," Circulation, 102(12), pp. 1364-1368.

[Lee, 2000] Lee, R., 2000, “Atherosclerotic lesion mechanics versus biology,” Z Kardiol. 89, pp. S080-S084.

[Li, 2009] Li N., Zhang H., and Ouyang H., 2009, "Shape optimization of coronary artery stent based on a parametric model," Finite Elements in Analysis and Design, 45, pp.468-475. [Lia, 2009] Li, N., Zhang, H., Ouyang, H., 2009, “Shape optimization of coronary artery stent based on a parametric model," Finite Elements in Analysis and Design, 45, 468-475.

[Liang, 2005] Liang, D.K., Yang, D. Z., Qi M., Wang W.Q., 2005, "Finite element analysis of the implantation of a balloon-expandable stent in a stenosed artery," International Journal of Cardiology, 104, pp. 314-318. 
[Lim, 2008] Lim, D., Cho, S., Park, W., Kristensson, A., Ko, J., Al-Hassani, S.T.S., and Kim, H., 2008, "Suggestion of potential stent design parameters to reduce restenosis risk driven by foreshortening or dog boning due to non-uniform balloon-stent expansion," Annals of Biomedical Engineering, 36 , pp. 1118-1129.

[Loree, 1994] Loree, H.M., Grodzinsky, A.J., Park S.Y., Gibson, L.J., and Lee, R.T., 1994, "Static circumferential tangential modulus of human atherosclerotic tissue," J Biomech. 27, pp. 195-204.

[LS Dyna Manual] LS Dyna Manual

[Lu, 2003] Lu, X., Yang, J., Zhao, J.B., Gregersen, H., and Kassab, G.S., 2003, “Shear modulus of porcine coronary artery: contributions of media and adventitia," Am J Physiol Heart Circ Physiol, 285, pp. H1966-H1975.

[Lu, 2004] Lu, X., Pandit, A., and Kassab, G.S., 2004, Biaxial incremental homeostatic elastic moduli of coronary artery: two-layer model. Am J Physiol Heart Circ Physiol 287: H1663H1669.

[Marrey, 2006] Marrey, R.V., Burgermeister, R., Grishaber, R.B., Ritchie, R.O., 2006,"Fatigue and life prediction for cobalt-chromium stents: A fracture mechanics analysis", Biomaterials, 27(9), pp. 1988-2000.

[Martin, 2002] Martin, H., Grabow, N., and Schmitz, K.P., 2002, "The impact of material characteristics on the mechanical properties of a poly (l-lactide) coronary stent," BiomedizinischeTechnik, Vol. 47, pp. 503-505.

[Martin, 2013] Martin, D. and Boyle, F. (2013). Finite element analysis of balloon-expandable coronary stent deployment: Influence of angioplasty balloon configuration. International Journal for Numerical Methods in Biomedical Engineering.

[Matweb, 2012] Young's Modulus, http://www.matweb.com. Last accessed January 9, 2012 [McGarry, 2004] McGarry, J.P., O’Donnell, B.P., McHugh, P.E., and McGarry, J.G., 2004, "Analysis of the mechanical performance of a cardiovascular stent design based on micromechanical modeling," Computational materials science, 31, pp. 421-438.

[Meng, 2006] Meng, B., Wang, J., Zhu, N., Meng, Q., Cui, F., and Xu, Y., 2006, "Study of biodegradable and self- expandable plla helical biliary stent in vivo and in vitro", Journal of Material Science, 17, pp.611-617. 
[Migliavacca, 2002] Migliavacca, F., Petrini, L., Colombo, M., Auricchio, F., and Pietrabissa, R., 2002, "Mechanical behaviour of coronary stents investigated through the finite element method," Journal of Biomechanics, 35, pp. 803-811.

[Migliavacca 2004] Migliavacca, F., Petrini, L., Massarotti, P., Schievano, S., Auricchio, F., and Dubini, G., 2004, "Stainless and shape memory alloy coronary stents: a computational study on the interaction with the vascular wall," Biomechanics and Modeling in Mechanobiology, 2(4), pp. 205-217.

[Migliavacca 2005] Migliavacca, F., Petrini, L., Montanari, V., Quagliana, I., Auricchio, F., and Dubini, G., 2005, "A predictive study of the mechanical behaviour of coronary stents by computer modelling," Medical Engineering and Physics, 27, pp.13-18.

[Migliavacca, 2007] Migliavacca, F., Gervaso, F., Prosi, M., Zunino, P., Minisini, S., Formaggia, L., and Dubini, G., 2007,“Expansion and drug elution model of a coronary stent,” Computer Methods in Biomechanics and Biomedical Engineering, 10(1), pp.63-73.

[Mori, 2005] Mori, K., and Saito, T., 2005, "Effect of stent structure on stent flexibility measurement," Annals of biomedical engineering, 33, pp. 733-742.

[Mortier, 2008] Mortier, P., De Beule, M., Carlier, S.G., Van Impe, R., Verhegghe, B., Verdonck, P., 2008, "Numerical study of the uniformity of balloon-expandable stent deployment," Journal of Biomechanical Engineering Vol. 130, pp.021018_1-021018_8 [Mortier, 2009] Mortier, P., De Beulea, M., Van Loob, D., Verhegghea, B., Verdonck, P., 2009, "Finite element analysis of side branch access during bifurcation stenting," Medical Engineering and Physics 31, pp. 434-440.

[Oberhofer, 2006] Oberhofer, G., Gese, H., Grob, M., Kuhling, M., and Seidel, D., 2006, "Numerical analysis of the balloon dilatation process using the explicit finite element method for the optimization of a stent geometry," in Proceedings of Ls-Dyna Anwender Forum, ULM 2006, pp. 35-46.

[Ozolanta, 1998] Ozolanta, I., Tetere, G., Purinya, B., and Kasyanov, 1998, "Changes in the mechanical properties, biochemical contents and wall structure of the human coronary arteries with age and sex," Medical Engineering and Physics, 20, pp. 523-533.

[Pant, 2012] Pant, S., Bressloff, N. W., and Limbert, G., 2012, "Geometry parameterization and multidisciplinary constrained optimization of coronary stents," Biomechanics and modeling in mechanobiology, 11(1-2), pp. 61-82. 
[Paszenda, 2005] Paszenda, Z., Walke, W., and Filipiak, J., 2005, "Experimental and numerical biomechanical analysis of vascular stent," Journal of materials processing technology, 164-165, pp. 1263-1268.

[Patel, 1970] Patel, D.J., and Janicki, J.S., 1970, "Static elastic properties of the left coronary circumflex artery and the common carotid artery in dogs," Circ Res, 27, pp.149-158.

[Paryab, 2012] Paryab, N., Ying, X., Boey, F. Y., Cronin, D., Lee-Sullivan, P., and Venkatraman, S., 2012, "Uniform expansion of a polymeric helical stent," ASME Journal of Medical Devices, 6(2), 021012.

[Paryab 2014] Paryab, N., Cronin, D. S., and Lee-Sullivan, P., 2014, "Finite element methods to analyze helical stent expansion," International journal for numerical methods in biomedical engineering, 30(3), pp. 339-352.

[Pericevic, 2009] Pericevic, I., Lally, C., Toner, D., Kelly, D.J., 2009, “The influence of plaque composition on underlying arterial wall stress during stent expansion: The case for lesion-specific stents," Medical Engineering and Physics, 31, pp. 428-433.

[Pizlo, 2001] Pizlo, I.G., Sawada, S., Wright, D., Segar, D., and Feigenbaum, H., 2001, "Detection of subclinical coronary atherosclerosis using two-dimensional high-resolution transthoracic echocardiography," Journal of the American College of Cardiology 37, pp. $1422-1429$.

[Prendergast, 2003] Prendergast, P.J., Lally, C., Daly, S., Reid, A. J., Lee, T.C., Quinn, D., and Dolan, F., 2003, "Analysis of prolapse in cardiovascular stents: a constitutive equation for vascular tissue and finite-element modelling,” J. Biomech. Eng., 125(5), 692-700.

[Regar, 2001] Regar, E., Sianos, G., and Serruys, P., 2001, "Stent development and local drug delivery," British Medical Bulletin, 59, pp.277-248.

[Rogers, 1999] Rogers, C., Tseng, D.Y., Squire, J.C., and Edelman, E.R., 1999, “Balloonartery interactions during stent placement," Circulation research, 84, pp. 378-383.

[Rosanio, 1999] Rosanio, S., tocchi, M., patterson, C., and Runge, M., 1999, "Prevention of restenosis after percutaneous coronary interventions,” Thromb Haemost, 82, pp. 164-167. [Schulze-Bauer, 2002] Schulze-Bauer C.A.J., Regitnig P., and Holzapfel G.A., 2002, "Mechanics of the human femoral adventitia including high-pressure response," Am J Physiol Heart Circ Physiol, 282, pp. H2427-H2440.

[Schwartz, 2002] Schwartz, R., and Henry, T., 2002, "Pathphysiology of coronary artery restenosis," Rev Cardiovas Med, 3, pp. 4-9. 
[Soares, 2010] Soares, J.S., Moore, J.E., Rajagopal, K.R., 2010,"Modeling of deformationaccelerated breakdown of polylactic acid biodegradable stents", Journal of Medical Devices, 4 , pp. 041007-1-10.

[Stoeckel, 2002] Stoeckel, D., Bonsignore, C., and Duda, S., 2002, “A survey of stent designs," Minimally Invasive therapy allied technologies, 11, pp. 137-147.

[Stolpmann, 2003] Stolpmann, J., Brauer, H., Stracke, H., Erbel, R., and Fischer, A., 2003, "Practicability and limitations of finite element simulation of the dilation behaviour of coronary stents," Material- Wissenschaft und Werkstofftech, 34, pp. 736-745.

[Stray, 1992] Stary, H. C., Blankenhorn, D. H., Chandler, A. B., Glagov, S., Insull Jr, W., Richardson, M., Rosenfeld, E., Schaffer, S. A., Schwartz, C. J., and Wagner, W. D., 1992, “A definition of the intima of human arteries and of its atherosclerosis-prone regions. A report from the Committee on Vascular Lesions of the Council on Arteriosclerosis," American Heart Association. Circulation, 85(1), pp.391.

[Su, 2003] Su, S. H., Chao, R. Y., Landau, C. L., Nelson, K. D., Timmons, R. B., Meidell, R. S., and Eberhart, R. C., 2003, "Expandable bioresorbable endovascular stent. i. fabrication and properties," Annals of biomedical engineering, 31(6), pp. 667-677.

[Tajaddini, 2005] Tajaddini, A., Kilpatrick, D.L., Schoenhagen, P., Tuzcu, E.M., Lieber, M., and Vince, D.G., 2005, "Impact of age and hyperglycemia on the mechanical behaviour of intact human coronary arteries: an ex vivo intravascular ultrasound study," American Journal of Physiology- Heart and Circulatory Physiology, 288, pp. H250-H255.

[Takashim, 2007] Takashim, K., Kitou, T., Mori, K., and Ikeuchi, K., 2007, "Simulation and experimental observation of contact conditions between stents and artery models," Medical Engineering and Physics, 29, pp.326-335.

[Tamura, 1996] Tamura, S., Hirao, M., Shiozaki, H., Inoue, M., Hashimoto, T., Hori, S., Ohkata, I., Asano, H., and Monden, M., 1996, “A newly-designed shape-memory coil stent for esophageal stricture: a preliminary report," Surgery Today, Japan Journal of Surgery, 26, pp. 945-948.

[Tan, 2001] Tan, L.B., Webb, D.C., Kormi, K., Al-Hassani, S.T.S., 2001, “A method for investigating the mechanical properties of intracoronary stents using finite element numerical simulation," International Journal of Cardiology, 78, pp.51-67. 
[Timmins, 2007] Timmins, L. H., Moreno, M. R., Meyer, C. A., Criscione, J. C., Rachev, A., and Moore Jr, J. E., 2007, "Stented artery biomechanics and device design optimization," Medical and biological engineering and computing, 45(5), pp. 505-513.

[Vaajanen, 2003] Vaajanen, A., Nuutinen, J. P., Isotalo, T., TÖRMÄLÄ, P., Tammela, T. L., and Talja, M., 2003, "Expansion and fixation properties of a new braided biodegradable urethral stent: an experimental study in the rabbit," The Journal of urology, 169(3), pp. 11711174.

[Vad, 2010] Vad, S., Eskinazi, A., Corbett, T., McGloughlin, T., \& Geest, J. P. V., 2010, "Determination of coefficient of friction for self-expanding stent-grafts," Journal of biomechanical engineering, 132(12), pp. 121007.

[van Andel, 2003] van Andel, C.J., Pistecky, P.V., Borst, C., 2003, "Mechanical properties of porcine and human arteries: implications for coronary anastomotic connectors," The Annals of thoracic surgery, 76, pp. 58-64.

[Vande Geest, 2004] Vande Geest, J.P., Sacks, M.S., and Vorp, D.A., 2004, “Age dependency of the biaxial biornechanical Behaviour of humian abdominal aorta," Journal of Biomechanical Engineering-Transactions of the Asme, 126, pp. 815-822.

[Venkatraman, 2003] Venkatraman, S., Lay Poh, T., Vinalia, T., Hou Mak, K., Boey, F., 2003, “Collapse pressures of biodegradable stents", Biomaterials, 24, pp. 2105-2111.

[Venkatraman, 2006A] Venkatraman, S., Tan, L., Joso, J., Boey, Y., and Wang, X., 2006, "Biodegradable stents with elastic memory," Biomaterials, 27, pp.1573-1578.

[Venkatraman, 2006B] Tan, L.P., Venkatraman, S.S., Joso, J.F.D., Boey, F.Y.C., 2006, “Collapse pressures of bilayered biodegradable stents", Inc. J Biomed Mater Res Part B: Applied Biomaterial, 79B, pp. 102-107

[Veress, 2000] Veress, A., Vince, D., Anderson, P.M., 2000, "Vascular mechanics of the coronary artery," ASAJO, 89, pp. II/92-II/100.

[Vito, 1982] Vito, R.P., and Demiray, H., 1982, “A two-layered model for arterial wall mechanics," Proc 35th Annu Conf Eng Med Biol.

[Wang, 2004] Wang, R., and Ravi-Chandar, K., 2004, "Mechanical response of a metallic aortic stent—part i: pressure-diameter relationship,” Journal of Applied Mechanics, 71, pp. 697.

[Vogt, 2004] Vogt, F., Stein, A., Rettemeier, G., Krottb, N., Hoffmann, R., vom Dahl, J., Bosserhoff, A., Michaeli, W., Hanrath, P., Weber, C., Blindt, R., 2004, “Long-term 
assessment of a novel biodegradable paclitaxel-eluting coronary polylactide stent," European Heart Journal, 25, pp. 1330-1340.

[Walke, 2005] Walke, W., Paszenda, Z., and Filipiak, J., 2005, "Experimental and numerical biomechanical analysis of vascular stent," Journal of Materials Processing Technology, 164165, pp. 1263-1268.

[Wang, 2006] Wang, W., Liang, D., Yang, D., and Qi, M., 2006, “Analysis of the transient expansion behaviour and design optimization of coronary stents by finite element method", Journal of Biomechanics Vol.39, pp. 21-32.

[Welch, 2008] Welch, T., Eberhart, R., and Chuong, C., 2008, "Characterizing the expansive deformation of a bioresorbable polymer fiber stent," Annals of Biomedical Engineering, 36, pp.742-751.

[Williams, 1999] Williams, M.J.A., Stewart, R.A.H., Low, C.J.S., and Wilkins, G.T., 1999, "Assessment of the mechanical properties of coronary arteries using intravascular ultrasound: an in vivo study," International Journal of Cardiac Imaging, 15, pp. 287-294.

[Wu, 2007] Wu, W., Wang, W.Q., Yang, D.Z., and Qi, M., 2007, "Stent expansion in curved vessel and their interactions: a finite element analysis," Journal of Biomechanics, 40, pp. 2580-2585.

[Wu, 2010] Wu, W., Petrini, L., Gastaldi, D., Villa, T., Vedani, M., Lesma, E., Prevital, B., and Migliavacca, F., 2010, "Finite element shape optimization for biodegradable magnesium alloy stents," Annals of Biomedical Engineering, 38, pp. 2829-2840.

[Yang, 2009] Yang, C., Bach, R. G., Zheng, J., Ei Naqa, I., Woodard, P. K., Teng, Z., Billiar, K., and Tang, D., 2009, “In vivo IVUS-based 3-D fluid-structure interaction models with cyclic bending and anisotropic vessel properties for human atherosclerotic coronary plaque mechanical analysis," Biomedical Engineering, IEEE Transactions on, 56(10), pp. 24202428..

[Yang, 2010] Yang, J., Liang, M.B., Huang, N., and Liux, Y.L., 2010, "Studying the nonuniform expansion of a stent influenced by the balloon," Journal of Medical Engineering and Technology, 34(5-6), pp. 301-305.

[Zahedmanesh, 2009] Zahedmanesh, H., and Lally, C., 2009, "Determination of the influence of stent strut thickness using the finite element method: implications for vascular injury and in-stent restenosis", Medical and Biological Engineering and Computing, 47, pp. 385-393. 
[Zahedmanesh, 2010] Zahedmanesh, H., Kelly, D., and Lally, C., 2010, "Simulation of a balloon expandable stent in a realistic coronary artery- Determination of the optimum modelling strategy," Journal of Biomechanics, 43, pp. 2126-2132. 